EDITORIAL BOARD

ANTONIO CARCATERRA

ERIC A. CARLEN

FRANCESCO DELL'ISOLA

RAFFAELE ESPOSITO

ALBERT FANNJIANG

Gilles A. FranCFORT

Pierangelo MARCATI

JEAN-JACQUES MARIGO

PETER A. MARKOWICH

MARTIN OSTOJA-STARZEWSKI

PIERRE SEPPECHER

DAVID J. STEIGMANN

PAUl STEINMANN

PierRe M. SuQueT

MANAGING EDITORS

MICOL AMAR

CORRADO LATTANZIO

ANGELA MADEO

MARTIN OSTOJA-STARZEWSKI

\section{ADVISORY BOARD}

ADNAN AKAY

Holm AltenBaCH

MICOL AMAR

HARM ASKES

TEODOR ATANACKOVIĆ

VICTOR BERDICHEVSKY

GUY BOUCHITTÉ

ANDREA BRAIDES

ROBERTO CAMASSA

MAURO CARFORE

ERIC DARVE

FELIX DARVE

ANNA DE MASI

GianPiEtro DEL Piero

EMMANUELE Di BENEDETTO

BERNOLD FIEDLER

IRENE M. GAMBA

DAVID Y. GAO

SERGEY GAVRILYUK

TIMOTHY J. HEALEY

DOMINIQUE JEULIN

ROGER E. KHAYAT

CORRADO LATTANZIO

ROBERT P. LIPTON

ANGELO LUONGO

ANGELA MADEO

JUAN J. MANFREDI

CARLO MARCHIORO

GÉRARD A. MAUGIN

ROBERTO NATALINI PATRIZIO NEFF

ANDREY PIATNITSKI

ERRICO PRESUTTI

MARIO PULVIRENTI

LUCIO RUSSO

Miguel A. F. SANJUAN

PATRICK SElvadurai

ALEXANDER P. SEYRANIAN

MIROSLAV ŠILHAVÝ

GUIDO SWEERS

ANTOINETTE TORDESILLAS

LEV TRUSKINOVSKY

JUAN J. L. VELÁZQUEZ VINCENZO VESPRI ANGELO VULPIANI msp.org/memocs

Università di Roma “La Sapienza”, Italia

Rutgers University, USA

(CO-CHAIR) Università di Roma "La Sapienza", Italia

(TREASURER) Università dell'Aquila, Italia

University of California at Davis, USA

(CO-CHAIR) Université Paris-Nord, France

Università dell'Aquila, Italy

École Polytechnique, France

DAMTP Cambridge, UK, and University of Vienna, Austria

(CHAIR MANAGING EDITOR) Univ. of Illinois at Urbana-Champaign, USA

Université du Sud Toulon-Var, France

University of California at Berkeley, USA

Universität Erlangen-Nürnberg, Germany

LMA CNRS Marseille, France

Università di Roma "La Sapienza", Italia

Università dell'Aquila, Italy

Université de Lyon-INSA (Institut National des Sciences Appliquées), France

(CHAIR MANAGING EDITOR) Univ. of Illinois at Urbana-Champaign, USA

Carnegie Mellon University, USA, and Bilkent University, Turkey

Otto-von-Guericke-Universität Magdeburg, Germany

Università di Roma "La Sapienza", Italia

University of Sheffield, UK

University of Novi Sad, Serbia

Wayne State University, USA

Université du Sud Toulon-Var, France

Università di Roma Tor Vergata, Italia

University of North Carolina at Chapel Hill, USA

Università di Pavia, Italia

Stanford University, USA

Institut Polytechnique de Grenoble, France

Università dell'Aquila, Italia

Università di Ferrara and International Research Center MEMOCS, Italia

Vanderbilt University, USA

Freie Universität Berlin, Germany

University of Texas at Austin, USA

Federation University and Australian National University, Australia

Université Aix-Marseille, France

Cornell University, USA

École des Mines, France

University of Western Ontario, Canada

Università dell' Aquila, Italy

Louisiana State University, USA

Università dell'Aquila, Italia

Université de Lyon-INSA (Institut National des Sciences Appliquées), France University of Pittsburgh, USA

Università di Roma "La Sapienza”, Italia

Université Paris VI, France

Istituto per le Applicazioni del Calcolo "M. Picone", Italy

Universität Duisburg-Essen, Germany

Narvik University College, Norway, Russia

Università di Roma Tor Vergata, Italy

Università di Roma "La Sapienza”, Italia

Università di Roma “Tor Vergata”, Italia

Universidad Rey Juan Carlos, Madrid, Spain

McGill University, Canada

Moscow State Lomonosov University, Russia

Academy of Sciences of the Czech Republic

Universität zu Köln, Germany

University of Melbourne, Australia

École Polytechnique, France

Bonn University, Germany

Università di Firenze, Italia

Università di Roma La Sapienza, Italia

MEMOCS (ISSN 2325-3444 electronic, 2326-7186 printed) is a journal of the International Research Center for the Mathematics and Mechanics of Complex Systems at the Università dell'Aquila, Italy.

Cover image: "Tangle” by $\odot$ John Horigan; produced using the Context Free program (contextfreeart.org).

PUBLISHED BY

7 mathematical sciences publishers

nonprofit scientific publishing

http://msp.org/

(C) 2017 Mathematical Sciences Publishers 


\title{
FORMS OF THE DISSIPATION FUNCTION FOR A CLASS OF VISCOPLASTIC MODELS
}

\author{
Massimo CuOmo
}

\begin{abstract}
The minimum properties that allow a dissipation functional to describe a behavior of viscoplastic type are analyzed. It is considered a material model based on an internal variable description of the irreversible processes and characterized by the existence of an elastic region. The dissipation functional derived includes the case of time-independent plasticity in the limit. The complementary dissipation functional and the flow rule are also stated. The model analyzed leads naturally to the fulfillment of the maximum dissipation postulate and thus to associative viscoplasticity. A particular class of models is analyzed, and similarities to and differences from diffused viscoplastic formats are given.
\end{abstract}

\section{Introduction}

Strain-rate-dependent behavior is characteristic of many materials at least beyond a certain level of stress, temperature or strain rate. Strain-rate sensitivity and timetemperature superposition effects occur when the time scale of the process is comparable with a characteristic relaxation time of the material. In this paper, we consider the case of a relatively short relaxation time characterizing irreversible phenomena such as plasticity and damage, leading to a generalized viscoplastic model. For such processes, irreversible deformations do not develop instantaneously, and also the apparent yield stress is modified according to the velocity of strain. The same observations apply to other phenomena like damage evolution or hardening, which can be described by additional internal variables in a similar way to the strain (see [Contrafatto and Cuomo 2002] for more details).

Viscoplasticity, introduced systematically by [Rabotnov 1969; Green and Naghdi 1965; Needleman 1988; Krempl 1975; Valanis 1971], to report only some of the earliest contributions to the subject, has received renewed attention in conjunction with the development of advanced models incorporating other phenomena, like hardening-softening behavior, nonassociative flow rules, anisotropy, etc. [Hall 2005; Phillips and Wu 1973; Zienkiewicz et al. 1975]. In most cases, an evolution

\section{Communicated by Francesco dell'Isola.}

MSC2010: 74A20, 74C10.

Keywords: dissipation potential, viscoplasticity, convex potentials. 
law for the anelastic variables is postulated, which satisfies the second law of thermodynamics, like was done for instance in the original Perzyna [1966] or Duvaut and Lions [1972] proposals, which are largely employed in numerical models. Thermodynamic reformulation of the generalized Perzyna and Duvaut-Lions models were also contributed [Ristinmaa and Ottosen 2000; 1998; Runesson et al. 1999; Perić 1993]. In these papers, the authors, in an attempt to better fit complex material behaviors, proposed an extension of the models using a dynamic yield locus, a function of the internal thermodynamic forces and/or of the viscoplastic strain rates, and a (complementary) dissipation function based on a decomposition of the conjugated forces. They showed that the formulation satisfies the dissipation inequality and that the postulate of maximum dissipation is fulfilled when an associated flow rule is used. Generalization to nonlocal models of plasticity have been proposed [Aifantis et al. 1999; Voyiadjis et al. 2004; Forest 2009], often as a mean for regularizing strain localization in softening materials. Gurtin [2003] proposed a framework for strain gradient small-deformation viscoplasticity. He introduces both polar stresses (third order) and microstresses (second order). However, both vanish when the dependency of the constitutive equations on the strain gradient is disregarded.

In this work, it is shown how viscoplastic constitutive relations can be consistently derived from a properly defined dissipation potential of the irreversible strain rates. The model is implemented within the generalized standard material model, in the definition given by Germain [1962] and Halphen and Nguyen [1975], which derives the constitutive laws from the specification of two potential functionals, the internal energy and the dissipation. Only in the second will an internal time scale be introduced, in order to model a viscoplastic-like behavior. In this way the dissipation inequality will be automatically fulfilled. Conjugated to the dissipation functional, a function of the plastic strain rate, is the complementary dissipation functional, a function of the internal thermodynamic forces. The latter allows one to obtain the flow rules for the plastic rates.

The objective of the paper is to state sufficient conditions for the dissipation functional in order to describe a viscoplastic-type behavior, which in addition admits the existence of an elastic region. Once these conditions will be stated, the complementary dissipation functional will be obtained and from it the flow rule for the internal variables. We will present a case for which the expressions derived can be obtained in a closed form. The answer to a similar question has been given in the case of inviscid plasticity [Eve et al. 1990; Romano et al. 1993]. Therefore, we wish for the dissipation potential for viscoplasticity to include the one for time-independent plasticity as a limit case. The analysis will be carried out in the hypothesis of small deformations so that the kinematic variables will be additively split into reversible and irreversible components. The irreversible component accounts both for time-dependent and time-independent strains. 
The main result of the paper is as follows. While a dissipation function for timeindependent plasticity has to be a positively homogeneous function (hodo) of the plastic strain rate, that is, the gauge function of a set of irreversible strain rates (that will be shown to be polar to the set of the elastic stresses), in order to obtain a time-dependent flow rule, it is necessary to add in the expression of the further dissipation terms, specifically positively homogeneous functions of degree $n>1$ ( $h o d n$ ), which will be recognized to be gauge-like functions. It will be shown that they are powers of the gauge function of a closed set, which again will be identified with the polar set of the elastic domain. Then an expression for the complementary dissipation functional in terms of the gauge function of the elastic domain will be derived. The result will be an overstress model of viscoplasticity.

In the framework of the thermomechanics of dissipative materials [Maugin 1999], the derivation of the constitutive equations from appropriate energy functionals is a standard procedure. However, especially in the case of time-dependent irreversible behavior, usually some specific form of the dissipation potential is postulated, derived from a known rheological model, like in [Houlsby and Puzrin 2002]. The result of this paper relative to a general form of a dissipation functional that gives rise to an overstress viscoplastic model appears new. Although the model is only sufficient for a time-dependent plastic evolution model, there are indications that it may be a general result. For instance, in a recent series of papers, Goddard [2014; Kamrin and Goddard 2014] derived viscoplastic dissipation potential for granular materials starting from Edelen's work [1973] on nonlinear generalization of the classical Rayleigh-Onsager dissipation potentials. In addition to prove a general form of symmetry relations, he presented a form of dissipation potentials for viscoplastic laws that turned out to be a homogeneous function of degree $n>1$. He also derived a complementary dissipation functional that is analogous to the one obtained in this work in the particular case that the dissipation function is given by only one term.

The theory in this paper is presented for the case of local models of deformation only. Its extension to higher gradient theories like those proposed in [dell'Isola et al. 2015; Placidi 2016; Neff et al. 2014] is possible although there are technical details that need to be carefully analyzed.

In the following section, the results anticipated in Section 1 will be systematically derived. Then a uniaxial example will be presented. Numerical results are not included in this work. The model obtained, also in the case when an explicit form of the flow rule cannot be stated, is amenable to a simple numerical treatment. A detailed examination of the numerical algorithm, which takes advantages of some results established in an earlier work [Contrafatto and Cuomo 2005] will be presented elsewhere.

The paper makes consistent use of convex analysis. Only sporadically are the introduced definitions explicitly stated. The reader can refer to standard texts of 
convex analysis for the details [Rockafellar 1970]. However, the most important mathematical definitions used in the paper are briefly reviewed in Appendices A and $\mathrm{B}$.

\section{Phenomenological constitutive model}

The viscoplastic material considered is characterized by the existence of an elastic domain such that no irreversible deformation is associated to stress states belonging to it. Plastic deformations occur otherwise. We consider the case that stresses beyond the elastic limit are allowed (overstress), in which case delayed plastic strains occur. Thus, the description does not cover all the models proposed for viscoplasticity, like power law models, etc.

The standard generalized material model introduced by [Germain 1962; Halphen and Nguyen 1975] is adopted, which can be synthetically described by the following assumptions.

(1) The equilibrium state of the system is described by a set of state variables, which include internal variables in addition to strain. The former account phenomenologically for the modification of the internal structure of the material and rule hardening, damage and other phenomena. In the present paper, the kinematic variables describing the state of the system will be collected in the vector $\eta$, which in general includes the macroscopic strain, and other variables, as described in [Contrafatto and Cuomo 2002]. In the present work, no specific constitutive model will be analyzed, so the variable $\eta$ will be left undefined.

(2) Each kinematic variable is decomposed into a reversible (elastic) and an irreversible part. In the paper, the linearized deformation theory is used so that an additive decomposition into an elastic recoverable part and an inelastic irrecoverable (plastic) strain is considered: $\eta=\eta_{e}+\eta_{p}$.

(3) The state of the system is determined by the functionals of the free energy and of the specific dissipation, $e\left(\eta_{e}\right)$ and $d\left(\dot{\eta}_{p}\right)$, the first a function of the reversible part of the internal variables and the second a function of the rate of their irreversible part only.

(4) By standard thermodynamic arguments, the internal driving forces, which in the paper are indicated by $\tau$ and which in general include stress and other thermodynamic forces dual to the internal variables, are obtained by differentiating the free energy,

$$
\tau=\partial_{\eta_{e}} e\left(\eta_{e}\right),
$$

where the symbol $\partial$ denotes subdifferentiation, in order to account for the common case of nonsmooth energy functionals. The internal forces are dual 
to the kinematic variables in the sense of the virtual power

$$
P_{i}=\langle\tau, \dot{\eta}\rangle,
$$

where the brackets denote the inner product in the appropriate vector space.

(5) Conjugated potentials are derivable through a Fenchel transformation. The dual potentials are indicated by the index " $c$ ":

$$
\begin{aligned}
e\left(\eta_{e}\right)+e^{c}(\tau) & =\left\langle\tau, \eta_{e}\right\rangle, & \tau \in \partial_{\eta_{e}} e\left(\eta_{e}\right), & \eta_{e} \in \partial_{\tau} e^{c}(\tau), \\
d\left(\dot{\eta}_{p}\right)+d^{c}(\tau) & =\left\langle\tau, \dot{\eta}_{p}\right\rangle, & \tau \in \partial_{\dot{\eta}_{p}} d\left(\dot{\eta}_{p}\right), & \dot{\eta}_{p} \in \partial_{\tau} d^{c}(\tau) .
\end{aligned}
$$

Sometimes in the paper, following a consolidated tradition in mathematical papers, instead of the index " $c$ ", the conjugated function to $f(x): X \rightarrow \mathbb{R}$ will be indicated by $f^{*}\left(x^{*}\right): X^{*} \rightarrow \mathbb{R}$. In the previous expressions, $X$ and $X^{*}$ are dual vector spaces.

\section{The dissipation potential}

The main results of the paper are presented in this section. First the case of rateindependent plasticity is examined, recalling classic results concerning the dissipation functional. Then they are generalized to the case of overstress models, in the hypotheses stated in Section 1. Throughout the paper, it will be assumed that the dissipation functional as well as the internal energy potential are convex functions. Nonconvex energy potentials, which have been introduced for several phenomena, are therefore excluded from the present treatment. The minimum properties required for a dissipation functional for reproducing a time-independent plastic behavior were stated in [Romano et al. 1993]; see also [Eve et al. 1990]. The key feature for obtaining time-independent plasticity is that the dissipation function, in addition to being subadditive, be positively homogeneous of degree 1 . It has been suggested that a characteristic relaxation time is introduced if the dissipation function is homogeneous of degree 2 in its argument [Maugin 1990]. The aim of this section is to analyze the requisites that give rise to a time-dependent dissipation.

The inviscid case. In order to examine the rate-independent case, the following statements are needed. Their proofs can be found in [Romano et al. 1993]. They follow from the results that a hodo proper convex function $f(x)$ is the support function of the set $C^{\circ}$, the polar of the closed convex set $C=\{x: f(x) \leq 1\}$, and that, since for a hodo function $f(0)=0$, the former set coincides with the subdifferential of the function at 0 .

Statement. If the dissipation function is sublinear (hodo and subadditive), then the thermodynamic forces $\tau$ given by (1) are such that

$$
\tau \in \partial d\left(\dot{\eta}_{p}\right) \subset K=\partial d(0)
$$


and

$$
d\left(\dot{\eta}_{p}\right)=\sup _{\tau \in k}\left\langle\tau, \dot{\eta}_{p}\right\rangle=\operatorname{supp} K \doteq \psi_{K}
$$

consequently, the conjugate dissipation potential is ( $K$ is convex and closed)

$$
d^{c}(\tau)=\operatorname{ind} K \text {. }
$$

In the previous statement, supp and ind denote the support and the indicator function of a convex set, respectively, and they are defined in Appendix B. In the paper, the support function is also denoted by $\psi$. A consequence of the above theorem is the following:

Corollary. For all elastic stress states, $d^{c}(\tau)=0$.

Proof. From Fenchel's equality, one has, assuming $\dot{\eta}_{p}=0$,

$$
\left\langle\tau, \dot{\eta}_{p}\right\rangle=0=d(0)+d^{c}(\tau)
$$

where the thermodynamic force is conjugated to the strain rate, that is, $\tau \in \partial d(0)$. The conclusion follows immediately recalling that $d(0)=0$.

It is useful to recall some results of convex analysis that apply to a convex hodo function, as is the case examined in this section.

First the concept of gauge is recalled. A gauge $\gamma(x \mid C)$ of a set $C$ is the function

$$
\gamma(x \mid C)=\inf \{\mu \geq 0: x \in \mu C\} .
$$

It can also be thought of as the positively convex hodo function generated by ind $C(x)+1$ (Figure 1, left). A gauge function is any function $k(x)$ such that $k(x)=\gamma(x \mid C)$ for some $C$. The set $C$ for which $k(x)$ is a gauge is exactly $C=\{x: k(x) \leq 1\}$.

Taking the conjugate of gauge functions of convex sets establishes a polarity correspondence between closed convex sets. A set $C^{\circ}$ is called the polar of $C$ if

$$
C^{\circ}=\left\{x^{*}: \operatorname{supp} C\left(x^{*}\right) \leq 1\right\}=\left\{x^{*}:\left\langle x, x^{*}\right\rangle \leq 1 \text { for all } x \in C\right\} .
$$

It is easy to see that, if $C$ is closed, convex and containing the origin, then the polar set $C^{\circ}$ is also closed, convex and contains the origin, and the gauge function of $C$ is the support function of $C^{\circ}$ and vice versa.

In order to extend the polarity correlation to more general convex functions, it is convenient to define the polar of a generic gauge $k(x)$ as

$$
\begin{aligned}
k^{\circ}\left(x^{*}\right) & =\inf \left\{\mu^{*} \geq 0: x^{*} \in \mu^{*} C^{\circ}\right\}=\inf \left\{\mu^{*} \geq 0:\left\langle x, x^{*}\right\rangle \leq \mu^{*} k(x) \text { for all } x\right\} \\
& =\sup _{x \neq 0} \frac{\left\langle x, x^{*}\right\rangle}{k(x)} .
\end{aligned}
$$



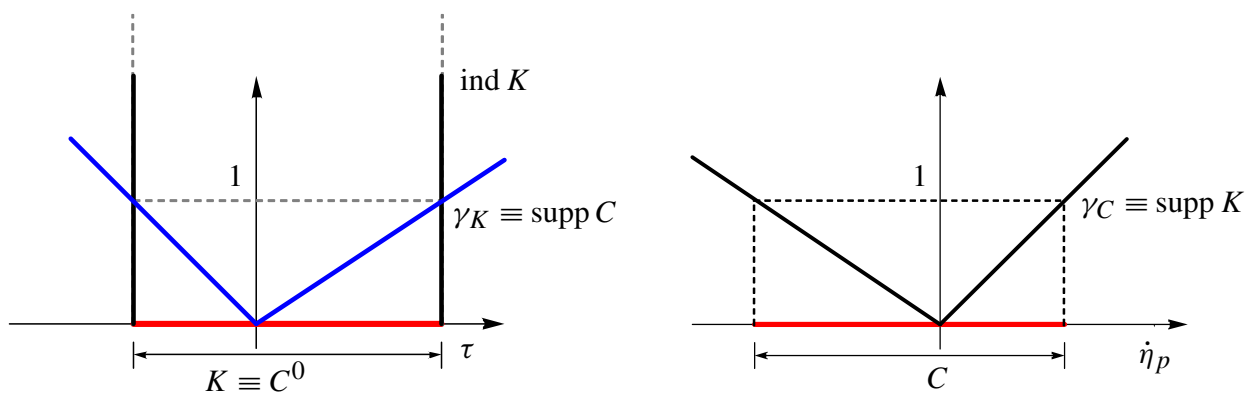

Figure 1. Gauge functions of the polar sets $C$ and $C^{\circ}$ and the indicator function of the set $K$. Left: the support function of the set $C$. Its level set at 1 defines the polar set $C^{\circ}$. Right: the support function of the set $K \equiv C^{\circ}$. Its level set at 1 defines the polar set $C$.

Then, denoting by $C$ and $C^{\circ}$ two closed polar sets containing the origin, we have the following polarity correspondences:

\begin{tabular}{|c|cc|}
\hline spaces & $C \subset X$ & $C^{\circ} \subset X^{*}$ \\
gauge functions & $k(x)$ & $k^{\circ}\left(x^{*}\right)$ \\
gauges & $\gamma(x \mid C)=\operatorname{supp} C^{\circ}(x)$ & $\gamma\left(x^{*} \mid C^{\circ}\right)=\operatorname{supp} C\left(x^{*}\right)$ \\
\hline
\end{tabular}

The connection to the plastic potential is readily established by the following:

Statement. The set $C=\left\{\dot{\eta}_{p}: d\left(\dot{\eta}_{p}\right) \leq 1\right\}=\left\{\dot{\eta}_{p}:\left\langle\tau, \dot{\eta}_{p}\right\rangle \leq 1\right.$ for all $\left.\tau \in K\right\}$ is polar to $K=\partial d(0)$.

Proof. Let $\tau \in K=\partial d(0)$. Then $\left\langle\tau, \dot{\eta}_{p}\right\rangle \leq d\left(\dot{\eta}_{p}\right)$ for all $\dot{\eta}_{p}$. In particular for $\dot{\eta}_{p} \in C$, $d\left(\dot{\eta}_{p}\right) \leq 1$, so $\tau \in C^{\circ}$.

The situation is represented in Figure 1 The gauge function of the set $K$ is the support function of the set $C$ whose level set at 1 is the elastic domain, and the gauge function of the set $C$ is the support function of the set $K$, that is, the dissipation function. The level set at 1 of the dissipation function is the set $C$. Therefore, for all the plastic strain rates belonging to the boundary of the set $C$, the rate of dissipation is the same. In the case of associated plasticity with a smooth yield function $g(\tau)-\sigma_{0} \leq 0$, the plastic strain rate is given by $\lambda \nabla g(\tau)$, and it is easy to see that the plastic strain rates belonging to $C$ are $\dot{\eta}_{p} \leq \nabla g / \sigma_{0}$.

The viscoplastic case. The statements above describe a model of inviscid plasticity with a yield function for the generalized stresses. In this section, we shall derive a form of the dissipation function that generalizes the one given in the previous section for inviscid plasticity. The derivation, whose technical details need some care, will be built in several steps. First it will be assumed that it can be assumed for the dissipation function a positively homogeneous function of degree larger than 1 . 
It will be shown that this kind of function is compatible with the mechanical model of a rate-dependent material, but it doesn't admit the existence of elastic states. A convenient form for this function will also be given. Then it will be shown that taking the dissipation function as the sum of a hodo function plus a function homogeneous of degree $n>1$ leads to describing the mechanical dissipation of a viscoplastic material with an elastic nucleus. Finally a general form for this class of dissipation functions will be proposed.

As stated above, let's assume that the dissipation function is convex and positively homogeneous of degree $n>1$ (hodn). Preliminarily, we prove the following:

Statement. For hodn (closed proper convex) dissipation functions $d_{n}$, with $n>1$, the set $\partial d_{n}(0)$ contains only the zero element.

Proof. By definition,

$$
\tau \in \partial d_{n}(0) \quad \Longleftrightarrow \quad\left\langle\tau, \dot{\eta}_{p}\right\rangle \leq d_{n}\left(\dot{\eta}_{p}\right) \text { for all } \dot{\eta}_{p} .
$$

Taking $\dot{\eta}_{p}=\mu \dot{\eta}_{p 0}, \mu \geq 0$, one has from (10)

$$
\mu\left\langle\tau, \dot{\eta}_{p 0}\right\rangle \leq \mu^{n} d_{n}\left(\dot{\eta}_{p 0}\right) \quad \text { for all } \mu
$$

and taking the limit as $\mu \rightarrow 0$, it follows that $\tau=0$.

The opposite implication is true only for a strictly convex dissipation function: Statement. If the dissipation potential, in addition to hodn, is strictly convex, then

$$
0 \in \partial d_{n}\left(\dot{\eta}_{p}\right) \quad \Longrightarrow \quad \dot{\eta}_{p}=0 .
$$

Proof. If $0 \in \partial d_{n}\left(\dot{\eta}_{p}\right)$, then $0 \leq d_{n}\left(\dot{\eta}_{p 0}\right)-d\left(\dot{\eta}_{p}\right)$ for all $\dot{\eta}_{p 0}$; that means that $d_{n}\left(\dot{\eta}_{p}\right)$ is a minimum for $d_{n}$, and since $d\left(\dot{\eta}_{p}\right) \geq 0$ for all $\dot{\eta}_{p}$, the statement follows from the strict convexity of $d_{n}$.

A nonnegative convex hodn function is in general not a gauge, so the results of the previous section related to the inviscid case do not apply. Therefore, more general convex functions conjugate to each other have to be introduced. A realvalued function $f$ is said to be gauge-like if $f(0)=0$ and the various level sets

$$
\{x: f(x) \leq \alpha\}, \quad f(0)<\alpha<+\infty,
$$

are all proportional, that is, are positive scalar multiples of a single set.

Lemma. A function $f_{n}$, positively homogeneous of degree $n, n>1$, is a gauge-like function.

Proof. Since $f_{n}$ is positively homogeneous of degree $n$, (a) $f_{n}(0)=0=\inf f_{n}$; introducing the notations $C_{n}=\left\{x: f_{n}(x) \leq 1\right\}$ and $C_{n p}=\left\{x: f_{n}(x) \leq p\right\}$,

$$
C_{n p}=\left\{x: f_{n}(x) \leq p\right\}=\left\{x: p^{-1} f_{n}(x) \leq 1\right\}=\left\{x: f_{n}\left(p^{-1 / n} x\right) \leq 1\right\},
$$


that is, (b) $C_{n p}=p^{1 / n} C_{n}$. Properties (a) and (b) ensure that $f_{n}$ is a gauge-like function.

A theorem of convex analysis [Rockafellar 1970, §13] states that a closed convex hodn function $f$ can always be expressed in the form

$$
f(x)=\frac{1}{n} k(x)^{n},
$$

where $k$ is the gauge of the closed set $C=\{x: k(x) \leq 1\}$ containing the origin, also known as the Minkowski function (as we have seen it is positively homogeneous, convex and such that $k(0)=0$ ).

Before proceeding further, it is convenient to introduce some definitions that allow us to use dimensionless quantities. Let $d_{0}=\tau_{0} v$, with $\tau_{0}$ a characteristic stress (which may be thought of as an equivalent limit stress), and $v=\dot{\eta}_{p 0}$ be the inverse of a characteristic time (with $\dot{\eta}_{p 0}$ an equivalent strain rate). Setting $\hat{\tau}=\tau / \tau_{0}, \hat{\dot{\eta}}_{p}=\dot{\eta}_{p} / \dot{\eta}_{p 0}$ and $\hat{d}=d / d_{0}$, it follows that $C=\left\{\hat{\dot{\eta}}_{p}: \hat{d}\left(\hat{\dot{\eta}}_{p}\right) \leq 1\right\}=$ $\left\{\dot{\eta}_{p}: d\left(\dot{\eta}_{p}\right) \leq d_{0}\right\}$. With these notations, the gauge function of $C, k_{C}\left(\dot{\eta}_{p}\right)$, which as observed on page 223 is equal to the support function of the polar set to $C$, $C^{\circ}=\left\{\hat{\tau}:\left\langle\hat{\tau}, \hat{\dot{\eta}}_{p}\right\rangle \leq 1\right.$ for all $\left.\hat{\dot{\eta}}_{p} \in C\right\}=\left\{\tau:\left\langle\tau, \dot{\eta}_{p}\right\rangle \leq d_{0}\right.$ for all $\left.\dot{\eta}_{p} \in C\right\}$, which is equal to the elastic domain $K$ (see the statement on page 223), becomes

$$
k_{C}\left(\dot{\eta}_{p}\right)=\operatorname{supp} C^{\circ}=\sup _{\hat{\tau} \in C^{\circ}}\left\langle\hat{\tau}, \hat{\dot{\eta}}_{p}\right\rangle=\sup _{\tau \in C^{\circ}} \frac{\left\langle\tau, \dot{\eta}_{p}\right\rangle}{d_{0}}=\frac{\operatorname{supp} K}{d_{0}} \doteq \frac{\psi_{K}\left(\dot{\eta}_{p}\right)}{d_{0}},
$$

where the symbol $\psi_{K}$ denoting the support function of the set $K$ has been introduced for brevity. Similarly, the polar gauge $k^{\circ}(\tau)$ to $C^{\circ}$ will be denoted by $j_{K}(\tau)=\operatorname{supp} C$, which with the notations introduced is equal to

$$
j_{K}(\tau)=\sup _{\dot{\eta}_{p} \in C} \frac{1}{d_{0}}\left\langle\tau, \dot{\eta}_{p}\right\rangle .
$$

Based on the above lemma, the characterization of the dissipation and of the complementary dissipation functionals is given in the following statement.

Statement. A positively homogeneous of degree $n, n>1$, dissipation function (that is, gauge-like), is given by the form

$$
d_{n}\left(\dot{\eta}_{p}\right)=\frac{1}{n} \frac{\left[\psi_{K}\left(\dot{\eta}_{p}\right)\right]^{n}}{d_{0}^{n-1}},
$$

and the conjugated complementary dissipation function is

$$
d_{n}^{c}(\tau)=\frac{n-1}{n} d_{0}\left[j_{K}(\tau)\right]^{n /(n-1)}
$$


Proof. The form (16) follows directly from (13) and (14), the latter giving the gauge function of the set $C$. In order to prove (17), preliminarily the conjugated function to $(1 / n) \psi_{K}^{n}$ is evaluated. By definition,

$$
\begin{aligned}
\left(\frac{1}{n} \psi_{K}^{n}\right)^{c} & =\sup _{\dot{\eta}_{p}}\left\{\left\langle\tau, \dot{\eta}_{p}\right\rangle-\frac{1}{n}\left[\psi_{k}\left(\dot{\eta}_{p}\right)\right]^{n}\right\} \\
& =\sup _{\dot{\eta}_{p}}\left\{\left\langle\tau, \dot{\eta}_{p}\right\rangle-\frac{1}{n}\left[\inf _{\mu \geq 0} \mu: d\left(\dot{\eta}_{p}\right) \leq \mu d_{0}\right]^{n}\right\} \\
& =\sup _{\dot{\eta}_{p}}\left\{\left\langle\tau, \dot{\eta}_{p}\right\rangle-\frac{1}{n}\left[\inf _{\mu \geq 0} \mu: \dot{\eta}_{p} \in \mu C\right]^{n}\right\}=\sup _{\mu \geq 0} \sup _{\dot{\eta}_{p} \in \mu C}\left\{\left\langle\tau, \dot{\eta}_{p}\right\rangle-\frac{1}{n} \mu^{n}\right\} \\
& =\sup _{\mu \geq 0}\left\{\mu\left(\sup _{\dot{\eta}_{p} \in C}\left\langle\tau, \dot{\eta}_{p}\right\rangle\right)-\frac{1}{n} \mu^{n}\right\}=\sup _{\mu \geq 0}\left\{\mu j_{K}(\tau)-\frac{1}{n} \mu^{n}\right\} \\
& =\frac{n-1}{n} j_{K}^{n /(n-1)} .
\end{aligned}
$$

Next, observing that $(\lambda f(x))^{c}=\lambda f^{c}\left(x^{*} / \lambda\right)$ and the fact that $\psi_{K}$ is a positively homogeneous function, we finally obtain

$$
\begin{aligned}
d_{n}^{c} & =\left(\frac{1}{n} \frac{\psi_{K}^{n}}{d_{0}^{n-1}}\right)^{c}=\frac{1}{d_{0}^{n-1}} \frac{n-1}{n}\left[j_{K}\left(\tau d_{0}^{n-1}\right)^{n /(n-1)}\right] \\
& =\frac{n-1}{n} d_{0}\left[j_{K}(\tau)\right]^{n /(n-1)} .
\end{aligned}
$$

So if the dissipation potential $d_{n}$ is positively homogeneous of degree $n$, its conjugate is positively homogeneous of degree $m=n /(n-1)$, with $1 / n+1 / m=1$.

Summarizing, it has been found that, if the dissipation function is a positively hodn, the only stress state conjugated to zero dissipation is zero, that is, the elastic domain reduces to the zero element alone. For any other stress state, the rate of plastic deformation is given by

$$
\dot{\eta}_{p} \in \partial_{\tau} \frac{n-1}{n}\left[k^{\circ}(\tau)\right]^{n /(n-1)}=\left[k^{\circ}(\tau)\right]^{1 /(n-1)} \partial_{\tau} k^{\circ}(\tau),
$$

that is, the rate of plastic deformation is proportional to a gauge; therefore, an overstress effect is found. The larger $n$ is, the smaller the plastic rate is. From Fenchel's identity, it can also be obtained that, if $\tau$ and $\dot{\eta}_{p}$ are a conjugated pair, the strain rate associated to an internal force $p \tau$ is $p^{1 /(n-1)} \dot{\eta}_{p}$. Only the case $n=2$ yields proportional strain rates; in this case, both the dissipation function and the conjugated dissipation $d_{n}^{c}$ are positively homogeneous of degree 2 .

In order to obtain a viscoplastic model with a threshold value for the stress and that reduces to inviscid plasticity as the relaxation time vanishes, the dissipation functional may then be taken as a sum of closed convex proper hodn functions for increasing values of $n \geq 1$. In this way, the dissipation function and, according 
to (17), also the complementary dissipation function are expressed as series expansions. In consideration of the finding that, if $d_{n}$ is positively homogeneous of degree $n, d_{n}^{c}$ is positively homogeneous of degree $n /(n-1)$, two series expansions are considered for the dissipation functional, which can both be expressed in the form

$$
d\left(\dot{\eta}_{p}\right)=\psi_{K}\left(\dot{\eta}_{p}\right)+\sum_{n=2}^{N} \frac{1}{n} \frac{1}{\left(\tau_{0} v\right)^{q-1}}\left[\psi_{K}\left(\dot{\eta}_{p}\right)\right]^{q}
$$

with

$$
q=n \quad \text { or } \quad q=\frac{n}{n-1} .
$$

In the following development, the choice will be left unspecified, and only in the final example will the two cases be differentiated. In the expression of the dissipation appears the sum of a hodo sublinear functional, and of other hodn terms, with $n>1$.

Remark. For the dissipation function defined by $(20), \partial d(0)=\partial \psi_{K}(0)=K$.

Proof. The observation follows from the fact that the functional (20) is the sum of proper convex functions, the relative interior of the domain of which have common points. In these hypotheses, one has $\partial d(0)=\partial \psi_{K}(0) \cup \partial \psi_{K}^{2}(0) \cup c \cup \cdots$, but $\partial \psi_{K}^{n}(0)=\{0\}, n>1$.

It is now possible to proceed to evaluate the conjugate dissipation function. Since the relative interiors of the domains of the addends of the dissipation function have obviously common points, the subgradient of the function (20) is given by the infimal convolution of the addends. Recalling that $\psi_{K}^{c}=$ ind $K$, using (17),

$$
d^{c}(\tau)=\inf \left\{\text { ind } K\left(\tau_{1}\right)+\sum_{n=2}^{N} \tau_{0} \nu \frac{q-1}{q}\left[j_{K}\left(\tau_{n}\right)\right]^{q /(q-1)}: \sum_{n=1}^{N} \tau_{n}=\tau\right\} .
$$

Some particular cases are examined. If $N=2$ and $q=n,(22)$ becomes $d^{c}(\tau)=\inf \left\{\operatorname{ind} K\left(\tau_{1}\right)+\frac{1}{2} \tau_{0} v j_{K}\left(\tau_{2}\right)^{2}: \tau_{1}+\tau_{2}=\tau\right\}=\frac{1}{2} \tau_{0} v \inf \left\{j_{K}\left(\tau-\tau_{1}\right)^{2}: \tau_{1} \in K\right\}$.

The infimum in (23) is the square of the minimum distance between the vector of the internal forces and the admissible domain in the norm induced by $j_{K}$.

In the case when $N=p$ and $q=n$ and all terms but the first and the $p$-th are null, one has

$$
\begin{aligned}
d^{c}(\tau) & =\inf \left\{\operatorname{ind} K\left(\tau_{1}\right)+\tau_{0} \nu \frac{p-1}{p} j_{K}\left(\tau_{2}\right)^{p /(p-1)}: \tau_{1}+\tau_{2}=\tau\right\} \\
& =\tau_{0} v \frac{p-1}{p} \inf \left\{j_{K}\left(\tau-\tau_{1}\right)^{p /(p-1)}: \tau_{1} \in K\right\} .
\end{aligned}
$$


In the general case, (22) can be rewritten as

$$
d^{c}(\tau)=\inf \left\{\sum_{n=2}^{N} \tau_{0} \nu \frac{q-1}{q} j_{K}\left(\tau_{n}\right)^{q /(q-1)}: \sum_{n=2}^{N} \tau_{n}=\bar{\tau}=\tau-\tau_{1} \tau_{1} \in K\right\},
$$

where $\bar{\tau}$ is the overstress.

The flow rule. The next step is to obtain an explicit form for the flow rule, which is done through the evaluation of the subgradient of the complementary dissipation function, which gives the set of the irreversible strain rates compatible with the constitutive equation.

In order to be specific, we examine the particular case that the function $d^{c}$ is given by (23) or (24). Since the function inside the infimum operation is positive, it is possible to interchange the power with the infimum operation so that, applying the chain rule of subdifferentiation,

$$
\dot{\eta}_{p} \in \partial d^{c}(\tau)=\tau_{0} v \inf \left\{j_{K}\left(\tau-\tau_{1}\right)^{1 /(p-1)}: \tau_{1} \in K\right\} \partial \xi(\tau),
$$

having indicated with $\xi$ the infimum of the gauge function

$$
\xi(\tau)=\inf \left\{j_{K}\left(\tau-\tau_{1}\right): \tau_{1} \in K\right\} .
$$

For evaluating its subdifferential, it is first observed that, if $\tau \in K, \xi(\tau)=0$; hence, $\partial \xi(\tau)=0$. If $\tau \notin K$, then one has $j_{k}(\tau)=\mu_{\tau} \geq 1$. Set $\tau_{10}=\mu_{\tau}^{-1} \tau$ so that $j_{K}\left(\tau_{10}\right)=1, \tau_{10} \in \partial K$, the boundary of $K$. The infimum operation in (27) can then be rewritten as

$$
\inf \left\{j_{K}\left(\mu_{\tau} \tau_{10}-\tau_{10}-\bar{\tau}_{1}\right): \tau_{10}+\bar{\tau}_{1} \in K\right\},
$$

where the vector $\bar{\tau}_{1}$ must be such that

$$
\left\langle\bar{\tau}_{1}, \dot{\eta}_{p}\right\rangle \leq 0 \quad \text { for all } \dot{\eta}_{p} \in N_{K}\left(\mu_{\tau}^{-1} \tau\right)=N_{\mu_{\tau} K}(\tau),
$$

$N_{K}$ being the tangent cone to $K$ at the point $\tau_{10}$.

From the convexity of $K$, it follows that the infimum in (28) is attained for $\bar{\tau}_{1}=0$ so that $\inf \left\{j_{K}\left(\tau-\tau_{1}: \tau_{1} \in K\right\}=j_{K}\left(\mu_{\tau} \tau_{10}-\tau_{10}\right)=\mu_{\tau}-1\right.$.

Statement. The subdifferential of the function $\xi(\tau)$ is given by

$$
\partial \xi(\tau)=\frac{1}{\tau_{0}} \gamma, \quad \gamma \in N_{K}\left(\mu_{\tau}^{-1} \tau\right)
$$

where $N_{K}\left(\mu_{\tau}^{-1} \tau\right)$ is the normal cone to $K$ at the point $\tau / \mu_{\tau}$.

Proof. The normal cone to $K$ at $\mu_{\tau}^{-1} \tau$ is

$$
N_{K}\left(\mu_{\tau}^{-1} \tau\right)=\left\{\dot{\eta}_{p}:\left\langle\dot{\eta}_{p}, \bar{\tau}-\frac{\tau}{\mu_{\tau}}\right\rangle \leq 0 \text { for all } \bar{\tau} \in K\right\} .
$$


By definition, the subdifferential of $\xi$ is

$$
\partial \xi(\tau)=\left\{\frac{1}{\tau_{0}} \hat{\dot{\eta}}_{p}:\left\langle\hat{\dot{\eta}}_{p}, \bar{\tau}-\hat{\tau}\right\rangle \leq \xi(\bar{\tau})-\xi(\tau)=\mu_{\bar{\tau}}-\mu_{\tau}\right\} .
$$

Dividing by $\mu_{\tau}$, one has

$$
\mu_{\tau}^{-1}\left\langle\hat{\dot{\eta}}_{p}, \bar{\tau}-\tau\right\rangle \leq \mu_{\bar{\tau}} / \mu_{\tau}-1
$$

and the last difference is smaller than 0 if $\bar{\tau} / \mu_{\tau} \in K$.

Then from (26), the rate of plastic deformation can be represented as

$$
\dot{\eta}_{p}=v\left(\mu_{\tau}-1\right)^{1 /(p-1)} \gamma, \quad \gamma \in N_{K}\left(\mu_{\tau}^{-1} \tau\right) .
$$

In this way, the flow rule has been characterized.

Corollary. The plastic strain rates in the case when the function $d^{c}$ is given by (23) or (24) are elements of the normal cone to $K$ at the point $\tau / \mu_{\tau}$.

A similar conclusion holds for the more general expressions of the dissipation potential as a power expansion, similar to what has been suggested by Goddard [2014]. The general case will be examined in a forthcoming paper.

Remark. According to (26) and (31), the viscoplastic strain rate is normal to the static yield surface at the closest point projection of the current stress state, where the definition of the closest point projection is in the sense of the Minkowski norm. This model, thus, does not include the generalization of the Duvaut-Lions model proposed by Simo [Simo and Govindjee 1991; Simo et al. 1988], which uses as the norm the complementary elastic energy $e^{c}\left(\tau-\tau_{1}\right), \tau_{1} \in K$.

Remark. The model obtained is associative, in the sense of the above corollary. Furthermore, it can be immediately applied to the case of hardening plasticity coupled with damage, once a generalized yield domain is defined, as proposed in [Contrafatto and Cuomo 2002]. The choice of the dissipation potential is completely independent of that of the internal energy. Notice that in the present model the same viscosity constant applies to the plastic strain rate and to the rate of the hardening variable. In order to model different time scales for the two phenomena, it would be necessary to introduce two different dissipation functions, both of the type (20): one for the plastic strain rate and the other for the rate of the plastic hardening. Investigating this case is however beyond the limits of this work.

Remark. In the case when the elastic domain $K$ has corner points, they are reflected in the flow rule as indicated by the inclusion of (31). The case of inviscid plasticity is naturally recovered when the relaxation time vanishes. 
In order to obtain an explicit expression for the complementary dissipation potential and for the flow rule to be more convenient for algorithmic developments, we introduce the classical yield function. In the continuum mechanics practice, the elastic domain $K$, rather than being defined as the polar set to $C=\left\{\hat{\dot{\eta}}_{p}: \hat{d}\left(\hat{\dot{\eta}}_{p}\right) \leq 1\right\}$, is directly introduced as the level set of a function $g(\tau)$, which possesses the properties

(1) inf $g=g(0)$ and

(2) the level sets $\{\tau: g(\tau) \leq c\}, g(0) \leq c \leq+\infty$, are all proportional.

Properties (1) and (2), using the result of the lemma on page 224, ensure that the yield function is a gauge-like function, so it has to be of the form $h(k(\tau))$, with $k(\tau)$ the gauge function. In particular it can be obtained as the composition of the gauge function of $K$ and of a nondecreasing, nonnegative, convex, lower semicontinuous function $h$ [Rockafellar 1970, Theorem 15.3]. However, it is convenient to take the function $g$ to be positively homogeneous, that is,

$$
K=\left\{\tau: j_{K}(\tau) \leq 1\right\}=\left\{\tau: g(\tau) \leq \tau_{0}\right\} \quad \Longrightarrow \quad g(\tau)=\tau_{0} j_{K}(\tau),
$$

where $\tau_{0}$ is the level of $g$ corresponding to the boundary of the set $K$. Then

$$
\xi(\tau)=\inf \left\{g\left(\tau-\tau_{1}\right) / \tau_{0}: \tau_{1} \in K\right\}=\left(g(\tau) / \tau_{0}-1\right)_{+}
$$

with $(x)_{+}=(x+|x|) / 2$. The subgradient $\partial \xi(\tau)$ can then be evaluated as $\partial \xi(\tau)=$ $\partial g(\tau) / \tau_{0}$ so that, if the function $g$ is differentiable at $\tau$, then the subgradient is composed by a unique vector, representing the outward normal to $\mu_{\tau} K$ in $\tau$, coinciding with the normal to $K$ in $\tau / \mu_{\tau}$. More generally, if $g(\tau)=\sup _{i} g_{i}(\tau)$, with each $g_{i}$ supposed differentiable, then if $\tau / \mu_{\tau}$ is a corner point of $K$, the subgradient is the convex combination of the normals $\partial g_{i}$ to $\mu_{\tau} K$ in $\tau$.

The flow rule, in the case when the dissipation function is given by (24), is then expressed as

$$
\dot{\eta}_{p}=\partial d_{n}^{c}(\tau)=v\left[\frac{g(\tau)-\tau_{0}}{\tau_{0}}\right]_{+}^{1 /(p-1)} \partial g(\tau) .
$$

Remark. Equation (35) in the case $p=2$ coincides with the formulation of Perzyna, the contents of the brackets being the overstress function.

Remark. From (35) it is observed that, using for the dissipation a power function greater than 2 of the support function of $K$, the complementary functional is a power function less than 2 of the Minkowski distance from the admissible domain. This can be interpreted as a stress dependency for the relaxation time (viscosity parameter). 


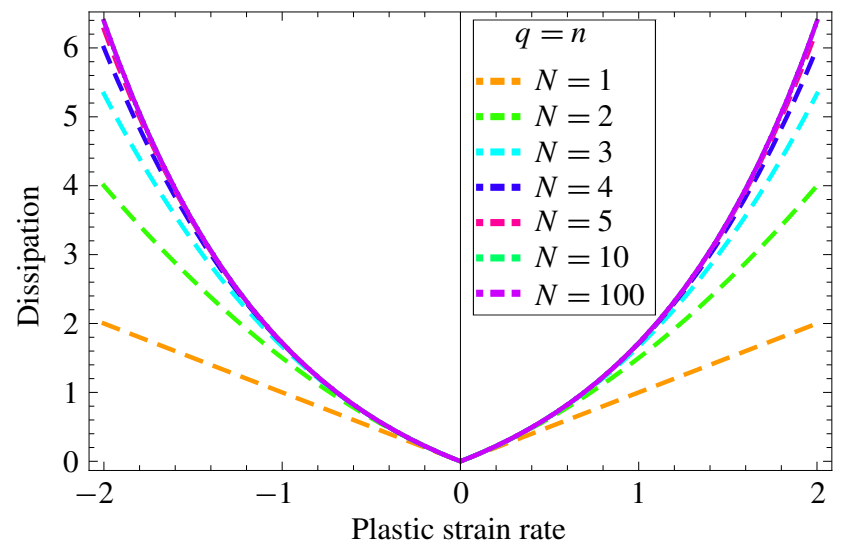

Figure 2. Dissipation function (36) for subsequent truncations of the series expansion.

\section{Uniaxial exemplification}

The main results of the previous section are now summarized and graphically illustrated in reference to the uniaxial case.

A slightly different expression for the dissipation function with respect to (20) is considered:

$$
d\left(\dot{\eta}_{p}\right)=\psi_{K}\left(\dot{\eta}_{p}\right)+\sum_{n=2}^{N} \frac{1}{n !} \frac{1}{\left(\tau_{0} v\right)^{n-1}}\left[\psi_{K}\left(\dot{\eta}_{p}\right)\right]^{n} .
$$

The motivation for introducing the factorial of $n$ lies in the fact that the form (36) is the series expansion of $\exp \left[\psi_{K}\right]-1$.

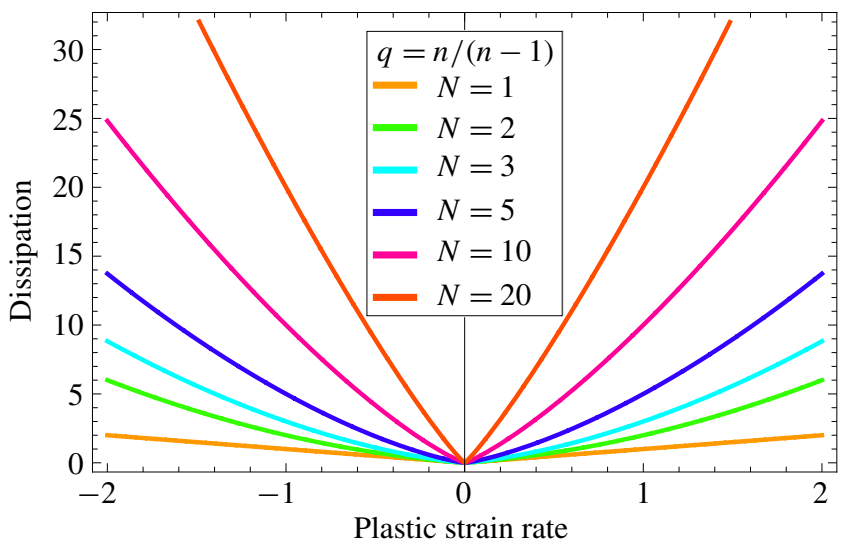

Figure 3. Dissipation function (20), $q=n /(n-1)$, for subsequent truncations of the series expansion. 


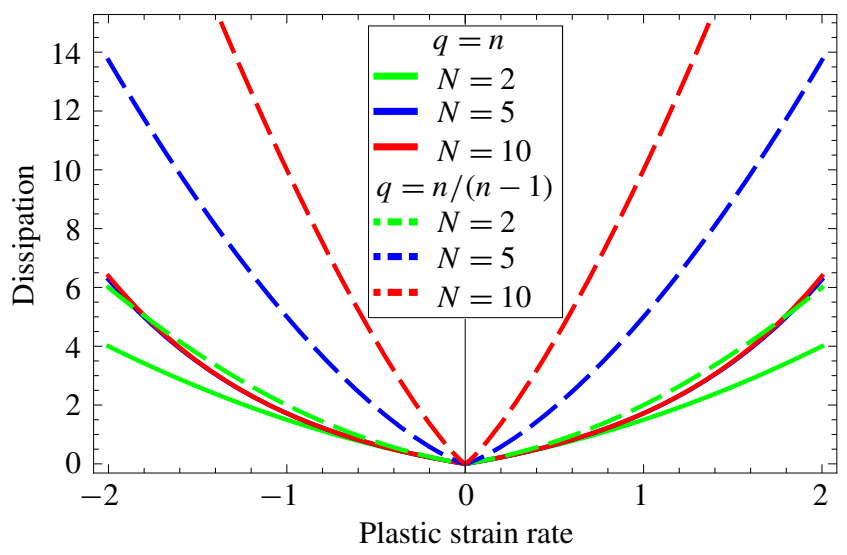

Figure 4. Comparison of the dissipation functions, (36) (solid lines) and (20) for $q=n /(n-1)$ (dashed lines).

Figure 2 shows the dissipation in an uniaxial case with $\tau_{0}=1$. It can be observed that, adding terms to the series for larger $N$, the dissipation function tends to converge to a limit value. The case given by (20) with $q=n /(n-1)$ is represented in Figure 3. In this case, adding terms to the series, the dissipation increases and tends toward a linear form, that is, the viscosity parameter tends to diverge, and a sort of inviscid plasticity is recovered for a wider elastic domain.

A comparison of the two forms is shown in Figure 4. The "exponential expansion" appears to yield lower values for the dissipation for the same $N$.

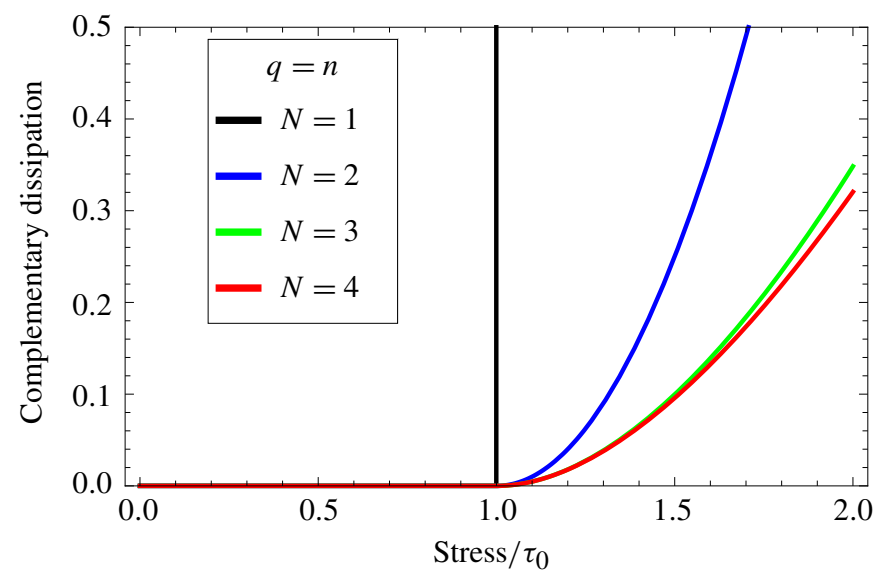

Figure 5. Complementary dissipation function (22) for the case $q=n /(n-1)$ for increasing number of functions in the series expansion. 
The complementary dissipation for the case (36) is given by

$$
d^{c}(\tau)=\inf \left\{\text { ind } K\left(\tau_{1}\right)+\sum_{n=2}^{N} \tau_{0} v \frac{n-1}{n}[(n-1) !]^{n /(n-1)}\left[j_{K}\left(\tau_{n}\right)\right]^{n /(n-1)}: \sum_{n=1}^{N} \tau_{n}=\tau\right\} .
$$

It is represented in the uniaxial case in Figure 5, where also the case of inviscid plasticity has been represented. Larger values of $N$ appear to act as mollifying parameters for the indicator function of the elastic domain.

\section{Conclusions}

The main results of the paper can be summarized as follows.

(1) We have given a formulation for the dissipation functional of a time-dependent dissipating material within the framework of the standard generalized material model; it has been shown that for the model to include an elastic domain the dissipation functional must be at least the sum of a positively homogeneous functional plus other hodn terms, with $n>1$. A form of the dissipation potential has been proposed, based on a sum of powers of the support function of the elastic domain, which degenerates into the dissipation function of timeindependent models when a viscosity parameter tends to 0 . This form is not unique, but it seems to be the simplest one compatible with the standard generalized material model that guarantees fulfillment of the dissipation inequality and that preserves all the essential properties of time-independent plasticity.

(2) The complementary dissipation functional, useful for the numerical implementation of the model, has been derived in a general form as the infimal convolution of gauge functions of the elastic domain. For the case that only two terms appear in the dissipation functional, and particularly for the commonly employed case that the second one is homogeneous of degree 2, the infimal convolution has been solved explicitly. Similarly, the relevant expressions for the flow rule have been derived.

(3) The general case of the dissipation function obtained as a power expansion of the support function of the elastic domain will be treated in a future paper. However, from a uniaxial exemplification, it seems that the series eventually converges to a limit form of the function.

\section{Appendix A: Homogeneous functions}

A function $f(x)$ is called positively homogeneous (of degree 1) (hodo) if

$$
f(\alpha x)=\alpha f(x), \quad \alpha \geq 0 .
$$


A function is called positively homogeneous of degree $n>1$ (hodn) if

$$
f(\alpha x)=\alpha^{n} f(x), \quad \alpha \geq 0 .
$$

In the text, the term "positively" will often be omitted for brevity.

\section{Appendix B: Review of some results of convex analysis}

Let $\{x \in X\}$ be a linear vector space. A function $f(x): X \rightarrow \overline{\mathbb{R}}$ is called convex if

$$
f\left((1-\lambda) x_{1}+\lambda x_{2}\right) \leq(1-\lambda) f\left(x_{1}\right)+\lambda f\left(x_{2}\right), \quad \lambda \in(0,1) .
$$

If the inequality in (40) is fulfilled strictly, the function is said to be strictly convex. The domain of $f$ is

$$
\operatorname{dom} f=\{x \in X: f(x)<+\infty\} .
$$

The function $f$ is said to be proper if $\operatorname{dom} f \neq \varnothing$ and $f(x)>-\infty$ for all $x \in X$.

Let $X$ be a topological real reflexive Banach space. The topological dual space to $X, X^{*}$, is the space of the linear functionals defined on $X$. The value of a functional $x^{*} \in X^{*}$ at $x$ is denoted by $\left\langle x^{*}, x\right\rangle$. If $X$ is a Hilbert space, then $\left\langle x^{*}, x\right\rangle$ is a scalar product and $X^{* *}=X$.

Let $f_{1}, f_{2}, \ldots, f_{n}$ be proper functions on a linear space $X$. The function

$$
f(x):=\inf \left\{f_{1}\left(x_{1}\right)+\cdots+f_{n}\left(x_{n}\right): x_{1}+\cdots+x_{n}=x, x_{i} \in X, i=1, \ldots, n\right\}
$$

is called the infimal convolution, and it is convex.

The support function of a convex set $K \subset X$ is the element of $X^{*}$

$$
\operatorname{supp} K=\sup _{y \in K}\{\langle y, x\rangle\}
$$

The indicator function of a set $A$ is

$$
\text { ind } A= \begin{cases}0 & \text { if } x \in A, \\ +\infty & \text { if } x \notin A .\end{cases}
$$

Given a set $C$, a gauge of the set $C$ is defined as

$$
\gamma_{C}(x)=\gamma(x \mid C)=\inf \{\mu: x \in \mu C, \mu \geq 0\},
$$

also called the Minkowski gauge functional.

A functional $f: X \rightarrow \overline{\mathbb{R}}$ is said to be sublinear if

(i) $f(\alpha x)=\alpha f(x)$ when $\alpha \geq 0$ (positive homogeneity) and

(ii) $f(x+y) \leq f(x)+f(y)$ (subadditivity).

A sublinear functional is a generalization of a norm on a linear space. 
A function $f(x)$ is said to be lower semicontinuous at $x_{0}$ if there exists a neighborhood $U_{0}(x)$ such that

$$
\text { there exists } \varepsilon>0 \quad \text { such that } f(x)-f\left(x_{0}\right)>\varepsilon \quad \text { for all } x \in U_{0}(x) .
$$

Subdifferential and conjugacy. Let $f: X \rightarrow \overline{\mathbb{R}}$ be a proper convex function. The subdifferential of $f$ at $x$ is the set

$$
\partial f(x)=\left\{x^{*} \in X^{*}: f(y)-f(x) \geq\left\langle x^{*}, y-x\right\rangle \text { for all } y \in X\right\} .
$$

The function $f^{*}\left(x^{*}\right): X^{*} \rightarrow \overline{\mathbb{R}}$ is called conjugate to $f(x): X \rightarrow \overline{\mathbb{R}}$ if

$$
f^{*}\left(x^{*}\right)=\sup _{x \in X}\left\{\left\langle x, x^{*}\right\rangle-f(x)\right\} .
$$

From the definition, it follows that

$$
f(x)+f^{*}\left(x^{*}\right) \geq\left\langle x, x^{*}\right\rangle .
$$

The equality sign in (49) holds only if $x^{*} \in \partial f(x)$.

\section{References}

[Aifantis et al. 1999] E. C. Aifantis, F. Oka, A. Yashima, and T. Adachi, "Instability of gradient dependent elasto-viscoplasticity for clay”, Int. J. Numer. Anal. Meth. Geomech. 23:10 (1999), 973994.

[Contrafatto and Cuomo 2002] L. Contrafatto and M. Cuomo, "A new thermodynamically consistent continuum model for hardening plasticity coupled with damage", Int. J. Solids. Struct. 39:25 (2002), 6241-6271.

[Contrafatto and Cuomo 2005] L. Contrafatto and M. Cuomo, "A globally convergent numerical algorithm for damaging elasto-plasticity based on the multiplier method", Int. J. Numer. Methods Eng. 63:8 (2005), 1089-1125.

[dell'Isola et al. 2015] F. dell'Isola, U. Andreaus, and L. Placidi, "At the origins and in the vanguard of peridynamics, non-local and higher-gradient continuum mechanics: an underestimated and still topical contribution of Gabrio Piola", Math. Mech. Solids 20:8 (2015), 887-928.

[Duvaut and Lions 1972] G. Duvaut and J.-L. Lions, Les inéquations en mécanique et en physique, Travaux et Recherches Mathématiques 21, Dunod, Paris, 1972.

[Edelen 1973] D. G. B. Edelen, "On the existence of symmetry relations and dissipation potentials", Arch. Rational Mech. Anal. 51:3 (1973), 218-227.

[Eve et al. 1990] R. A. Eve, B. D. Reddy, and R. T. Rockafellar, "An internal variable theory of elastoplasticity based on the maximum plastic work inequality", Quart. Appl. Math. 48:1 (1990), 59-83.

[Forest 2009] S. Forest, "Micromorphic approach for gradient elasticity, viscoplasticity, and damage", J. Eng. Mech. 135:3 (2009), 117-131.

[Germain 1962] P. Germain, Mécanique des milieux continus, Masson, Paris, 1962.

[Goddard 2014] J. D. Goddard, "Edelen's dissipation potentials and the visco-plasticity of particulate media”, Acta Mech. 225:8 (2014), 2239-2259. 
[Green and Naghdi 1965] A. E. Green and P. M. Naghdi, "A general theory of an elastic-plastic continuum", Arch. Rational Mech. Anal. 18:4 (1965), 251-281.

[Gurtin 2003] M. E. Gurtin, "On a framework for small-deformation viscoplasticity: free energy, microforces, strain gradients”, Int. J. Plasticity 19:1 (2003), 47-90.

[Hall 2005] R. B. Hall, "A thermodynamic framework for viscoplasticity based on overstress (VBO)", J. Eng. Mater. 127:4 (2005), 369-373.

[Halphen and Nguyen 1975] B. Halphen and Q. S. Nguyen, "Sur les matériaux standard généralisés", J. Mécanique 14 (1975), 39-63.

[Houlsby and Puzrin 2002] G. T. Houlsby and A. M. Puzrin, "Rate-dependent plasticity models derived from potential functions", J. Rheol. 46:1 (2002), 113-126.

[Kamrin and Goddard 2014] K. Kamrin and J. D. Goddard, "Symmetry relations in viscoplastic drag laws", Proc. R. Soc. Lond. A 470:2172 (2014), 20140434.

[Krempl 1975] E. Krempl, "On the interaction of rate and history dependence in structural metals", Acta Mech. 22:1 (1975), 53-90.

[Maugin 1990] G. A. Maugin, "Internal variables and dissipative structures", J. Non-Equil. Thermody. 15:2 (1990), 173-192.

[Maugin 1999] G. A. Maugin, The thermomechanics of nonlinear irreversible behaviors: an introduction, World Scientific Series on Nonlinear Science A 27, World Scientific, Singapore, 1999.

[Needleman 1988] A. Needleman, "Material rate dependence and mesh sensitivity in localization problems", Comput. Method. Appl. Mech. Eng. 67:1 (1988), 69-85.

[Neff et al. 2014] P. Neff, I.-D. Ghiba, A. Madeo, L. Placidi, and G. Rosi, "A unifying perspective: the relaxed linear micromorphic continuum", Contin. Mech. Thermodyn. 26:5 (2014), 639-681.

[Perić 1993] D. Perić, "On a class of constitutive equations in viscoplasticity: formulation and computational issues”, Int. J. Numer. Methods Eng. 36:8 (1993), 1365-1393.

[Perzyna 1966] P. Perzyna, "Fundamental problems in viscoplasticity", Adv. Appl. Mech. 9 (1966), 243-377.

[Phillips and Wu 1973] A. Phillips and H.-C. Wu, "A theory of viscoplasticity", Int. J. Solids Struct. 9:1 (1973), 15-30.

[Placidi 2016] L. Placidi, "A variational approach for a nonlinear one-dimensional damage-elastoplastic second-gradient continuum model”, Contin. Mech. Thermodyn. 28:1-2 (2016), 119-137.

[Rabotnov 1969] Yu. N. Rabotnov, Creep problems in structural members, North-Holland, Amsterdam, 1969.

[Ristinmaa and Ottosen 1998] M. Ristinmaa and N. S. Ottosen, "Viscoplasticity based on an additive split of the conjugated forces", Eur. J. Mech. A Solids 17:2 (1998), 207-235.

[Ristinmaa and Ottosen 2000] M. Ristinmaa and N. S. Ottosen, "Consequences of dynamic yield surface in viscoplasticity”, Int. J. Solids Struct. 37:33 (2000), 4601-4622.

[Rockafellar 1970] R. T. Rockafellar, Convex analysis, Princeton Mathematical Series 28, Princeton University, 1970.

[Romano et al. 1993] G. Romano, L. Rosati, and F. Marotti de Sciarra, "An internal variable theory of inelastic behaviour derived from the uniaxial rigid-perfectly plastic law", Int. J. Eng. Sci. 31:8 (1993), 1105-1120.

[Runesson et al. 1999] K. Runesson, M. Ristinmaa, and L. Mähler, "A comparison of viscoplasticity formats and algorithms", Mech. Cohes.-Frict. Mat. 4:1 (1999), 75-98. 
[Simo and Govindjee 1991] J. C. Simo and S. Govindjee, "Nonlinear B-stability and symmetry preserving return mapping algorithms for plasticity and viscoplasticity", Int. J. Numer. Methods Eng. 31:1 (1991), 151-176.

[Simo et al. 1988] J. C. Simo, J. G. Kennedy, and S. Govindjee, "Nonsmooth multisurface plasticity and viscoplasticity: loading/unloading conditions and numerical algorithms", Int. J. Numer. Methods Eng. 26:10 (1988), 2161-2185.

[Valanis 1971] K. C. Valanis, "A theory of viscoplasticity without a yield surface, I: General theory", Arch. Mech. Stos. 23 (1971), 517-533. In Polish.

[Voyiadjis et al. 2004] G. Z. Voyiadjis, R. K. Abu Al-Rub, and A. N. Palazotto, "Thermodynamic framework for coupling of non-local viscoplasticity and non-local anisotropic viscodamage for dynamic localization problems using gradient theory", Int. J. Plasticity 20:6 (2004), 981-1038.

[Zienkiewicz et al. 1975] O. C. Zienkiewicz, C. Humpheson, and R. W. Lewis, "Associated and nonassociated visco-plasticity and plasticity in soil mechanics", Géotechnique 25:4 (1975), 671-689.

Received 6 Jun 2016. Revised 16 Jan 2017. Accepted 10 Mar 2017.

MASSIMO CUOMO: mcuomo@dica.unict.it

Department of Civil Engineering and Architecture, University of Catania, Viale Andrea Doria 6, I-95125 Catania, Italy 



\title{
TUMOR GROWTH MODELLING BY CELLULAR AUTOMATA
}

\author{
RUBEN INTERIAN, REINALDO RODRÍGUEZ-RAMOS, \\ FERNANDO VALDÉS-RAVELO, ARIEL RAMÍREZ-ToRRES, \\ Celso C. Ribeiro and Aura Conci
}

\begin{abstract}
Tumor growth is a complex process that requires mathematical modeling approaches for studying real-life cancer behavior. The use of cellular automata (CA) to represent tumor growth in its avascular stage is explained in this work, and a stochastic CA describing tumor growth is obtained, based on a differential equations system in the range of continuum mechanics. The novelty of this research is the deduction of the neighborhood structure and rules for a probabilistic CA from these differential equations that describe the evolution of the tumor growth. In addition, the influence of the stresses on tumor growth is captured by the CA.
\end{abstract}

\section{Introduction}

Cell-based and cell-centered approaches for the study of biological soft tissues have been widely used [Hoehme and Drasdo 2010; Merks and Glazier 2005]. In particular, cellular automata (CA) are one of the most successful models [Rejniak and Anderson 2011; Boondirek et al. 2010] and have been used in a large number of studies [Kansal et al. 2000b; Dormann and Deutsch 2002; Deutsch and Dormann 2005].

Cellular automata models have been widely used to simulate avascular tumor growth [Dormann and Deutsch 2002; Kansal et al. 2000a], tumor cell invasion [Anderson et al. 2009], and tumor interactions with various environmental factors [Rejniak and McCawley 2010]. A review of the main methodologies for CA models describing tumor growth is provided in [Boondirek et al. 2010], emphasizing that most researchers have attempted to consider a microscopic scale to describe the macroscopic characteristics of tumor morphology. In [Kansal et al. 2000b], a three-dimensional cellular automaton model of brain tumor growth is developed, simulating the Gompertzian model very precisely. A quantitative analysis of the growth of a subpopulation within a previously homogeneous tumor is presented in

\section{Communicated by Francesco dell'Isola.}

MSC2010: 37B15, 68Q80, 74A10.

Keywords: tumor growth, stresses, stochastic rules, cellular automata. 
[Kansal et al. 2000a], applying a cellular automaton with a Delaunay triangulation lattice. In [Dormann and Deutsch 2002], avascular tumor growth is simulated using a hybrid lattice-gas cellular automaton, exhibiting self-organized formation of a layered tumor structure. A multiscale investigation focused on tumor cell invasion is given in [Anderson et al. 2009], using an evolutionary hybrid cellular automata model (EHCA). A comparison of relative strengths and weaknesses of various cellbased models, including CA, is presented in [Rejniak and McCawley 2010].

In this work, cellular automata are used for studying the process of tumor growth, specially in its avascular stage. The novelty of this research is the deduction of the neighborhood structure and the rules for a stochastic CA from continuous deterministic differential equation (DE) models. In addition, both the application of CA to study the evolution of tumor growth in time and the influence of the stresses in the growing of the tumor are considered in this work. The validation of the theoretical biomechanical model using CA is presented as well.

Having a CA model created from a system of differential equations allows one to describe more realistically the real-life situation of the tumor stage. The goals of the present work are summarized as follows:

(1) To visualize the tumor growth process represented by a system of differential equations.

(2) To obtain the evolution of the variables over time, mainly the tumor radius, in a new manner, more realistic and closer to the reality, where the tumor is not perfectly regular or circular. To reach this goal, a continuous deterministic DE model is transformed into a probabilistic CA.

(3) To create an alternative for studying the process of tumor growth in an interactive mode.

\section{Cellular automata}

Cellular automata have been seen mainly as discrete abstract computational systems. The Stanford Encyclopedia of Philosophy [Berto and Tagliabue 2012] defines cellular automata as follows:

Firstly, CA are (typically) spatially and temporally discrete: they are composed of a finite or denumerable set of homogeneous, simple units, the atoms or cells. At each time unit, the cells instantiate one of a finite set of states. They evolve in parallel at discrete time steps, following state update functions or dynamical transition rules: the update of a cell state obtains by taking into account the states of cells in its local neighborhood (there are, therefore, no actions at a distance). Secondly, CA are abstract, as they can be specified in purely mathematical terms and implemented 
in physical structures. Thirdly, CA are computational systems: they can compute functions and solve algorithmic problems. Despite functioning in a different way from traditional, Turing machine-like devices, CA with suitable rules can emulate a universal Turing machine, and therefore compute, given Turing's Thesis, anything computable.

Rigorously, the cellular automata is a quadruple $(C, n, S, f)$ [Deutsch and Dormann 2005], where:

- $C$ is a set of cells, not required to be finite.

- $n: C \times C \rightarrow\{0,1\}$ is a neighborhood function that can been seen as a relationship (usually reflexive and symmetric) between the cells. This function shows which pairs of cells are neighbors, that is, the geometry of the cell organization. Furthermore, $n$ must satisfy the neighborhood size independence condition: $\left|N\left(c_{0}\right)\right|=\left|\left\{c \in C: n\left(c_{0}, c\right)=1\right\}\right|=N$ is a constant for every $c_{0} \in C$; i.e., the size of the neighborhood is the same for all cells.

- $S$ is a set of states. As discussed below, each cell will have an associated state, in each moment.

- $f: S^{|N|} \rightarrow S$ is a transition function. The transition function is a core of the CA dynamics and is commonly expressed with rules that define the state of the cell in the next time moment from the state of the cell neighbors.

The set of cells $C$ with the neighborhood function $n$ defines the structure of the cell space.

The simplest CA model can have binary cells (two states, "tumoral" or "normal" [Hu and Ruan 2003]). Commonly, the states are represented by a set of integer values $\{0,1,2, \ldots\}$, each of these values having an appropriate physical or biological interpretation.

\section{Linear elasticity tumor model}

This work focuses on the mathematical model developed in [Ramírez-Torres et al. 2016], where the authors using linear elasticity generalize the Ngwa-Agyngi model [2012], which describes the evolution of growth-induced stresses in a spherical and isotropic growing tumor surrounded by an external medium.

The generalization of the model considering real cases of stresses is discussed in [Ramírez-Torres et al. 2016], where the dependence of tumor growth on the stresses is analytically derived. This particular model explores the avascular stage of a solid tumor.

This section briefly describes the model. We note that, in this article, we do not focus on its derivation but, instead, on obtaining from the model a CA that represents the tumor growth, as described in the next section. 


\begin{tabular}{|cl|}
\hline Variable & Definition \\
\hline$t$ & Time \\
$r$ & Radial coordinate \\
$R(t)$ & Radius of the tumor at time $t$ \\
$R_{0}$ & Radius of the tumor at $t=0$ \\
$c(r, t)$ & Nutrient concentration inside the tumor \\
$c_{b}$ & Nutrient concentration at boundary \\
$\boldsymbol{u}(r, t)$ & Tumor cell displacement \\
$\boldsymbol{v}(r, t)$ & Tumor cell displacement velocity \\
$\boldsymbol{\sigma}$ & Cauchy stress tensor of the tumor \\
$\sigma_{r}$ & Cauchy stress tensor component in radial direction \\
$\sigma_{\theta}$ & Cauchy stress tensor component in transversal direction \\
$\boldsymbol{\sigma}^{e}$ & Cauchy stress tensor of the external medium \\
$E$ & Young's modulus \\
$\nu$ & Poisson's ratio \\
$\boldsymbol{e}$ & Strain tensor of the tumor \\
$\boldsymbol{e}^{e}$ & Strain tensor of the external medium \\
$\rho$ & Tumor cell density \\
$\gamma_{r}$ & Tumor growth anisotropy parameter (radial direction) \\
$\gamma_{\theta}$ & Tumor growth anisotropy parameter (transversal direction) \\
$\eta_{1}$ & Dependence of cell proliferation on stresses \\
$\eta_{2}$ & Dependence of cell death on stresses \\
&
\end{tabular}

Table 1. Model variable definitions.

The variables and constants of the model are shown in Table 1.

The mathematical model obtained by [Ramírez-Torres et al. 2016] is a generalization of [Ngwa and Agyingi 2012] as a result of the followings assumptions:

(i) Tumor cells form a homogeneous population that is considered a continuum.

(ii) There is adhesion between tumor cells at the boundary, which maintains the tumor's solid shape and is in equilibrium with the expansive forces exerted by the internal cell proliferation.

(iii) The tumor has a spherical shape, and its symmetry is maintained at all times.

(iv) The tumor is in a state of diffusion equilibrium.

(v) The nutrient consumption rate is proportional to the nutrient concentration and to the tumor cell density. Without stresses, the cellular proliferation rate is proportional to the nutrient concentration and to the tumor cell density, while cell death is proportional to the cellular density.

(vi) The tumor material is assumed to be incompressible and responds to stress in a purely elastic and isotropic form.

(vii) There is a constant nutrient concentration in the tumor boundary. 
(viii) There is an external medium, which is supposed to be elastic, isotropic, and incompressible.

The tumor is modeled as a solid in the three-dimensional space, and the forces on it are considered acting per volume unit. As a result of the spherical symmetry hypothesis, the problem is treated in one dimension with respect to the radial coordinate $r$.

3.1. Kinematics and equilibrium equations. Because of the radial symmetry (hypothesis (iii)), the surface of the tumor is given by $S=r-R(t)$ and the velocity field has the form $\boldsymbol{v}=\left(v_{r}, 0,0\right)$, leading to

$$
\frac{d R}{d t}=v_{r}(R, t) \text {. }
$$

This equation represents the growth rate of the tumor. Removing the inertial factors and considering hypothesis (i), the equilibrium equation is

$$
\nabla \cdot \boldsymbol{\sigma}+\boldsymbol{F}=0
$$

where $\boldsymbol{F}$ is the vector of body forces, which is considered null.

3.2. Constitutive equation. The constitutive relation, which associates the stress $\sigma_{i j}$ with the material strain $e_{i j}$, represents a material with a linear elastic response subject to an anisotropic growth:

$$
e_{i j}=g\left(\delta_{1 i} \gamma_{r}+\left(\delta_{2 i}+\delta_{3 i}\right) \gamma_{\theta}\right) \delta_{i j}+\frac{1+v}{E} \sigma_{i j}-\frac{v}{E} \delta_{i j} \sigma_{k k} \quad \text { with } i, j, k=r, \theta, \phi,
$$

where $g$ is the growing factor, $\delta_{i j}$ is Kronecker's delta, $\gamma_{r}, \gamma_{\theta} \in \mathbb{R}_{+}$, and $\gamma_{r}+2 \gamma_{\theta}=1$. Parameters $\gamma_{r}$ and $\gamma_{\theta}$ represent the proportions of the tumor growth in the radial and transversal directions, respectively.

Assuming small deformations, $\boldsymbol{e}=\frac{1}{2}\left(\nabla \boldsymbol{u}+(\nabla \boldsymbol{u})^{T}\right)$, using the material incompressibility (i.e., $v=\frac{1}{2}$ ) from hypothesis (iv), and applying a Jaumann derivative in (3), we obtain the relationship between the rates of deformation and stress

$$
\frac{1}{2}\left(\nabla \boldsymbol{v}+(\nabla \boldsymbol{v})^{T}\right)_{i j}=(\nabla \cdot \boldsymbol{v})\left(\delta_{1 i} \gamma_{r}+\left(\delta_{2 i}+\delta_{3 i}\right) \gamma_{\theta}\right) \delta_{i j}+\frac{1}{2 E} \frac{D}{D t}(3 \boldsymbol{\sigma}-\boldsymbol{I} \operatorname{tr}(\boldsymbol{\sigma}))_{i j} .
$$

3.3. External medium. From assumption (viii), the external medium satisfies the generalized Hooke's law

$$
\sigma_{i j}^{e}=\frac{E v}{(1+v)(1-2 v)} \delta_{i j} e_{k k}^{e}+\frac{E}{1+v} e_{i j}^{e},
$$

since the material is incompressible, $v=\frac{1}{2}$, and (5) becomes

$$
\sigma_{i j}^{e}=-p \delta_{i j}+\frac{2 E}{3} e_{i j}^{e}
$$

where $p$ is the isotropic pressure. 
3.4. Growth equation. For a living tissue, growth can be interpreted as the difference between cell production and cellular death. Then, from the mass-conservation continuity equation and hypothesis (v), we have

$$
\underbrace{\frac{\partial \rho}{\partial t}+\nabla \cdot(\boldsymbol{v} \rho)}_{\text {growth }}=\underbrace{\alpha c \rho\left(1+\eta_{1} \operatorname{tr}(\boldsymbol{\sigma})\right)}_{\text {cellular proliferation }}-\underbrace{k \rho\left(1-\eta_{2} \operatorname{tr}(\boldsymbol{\sigma})\right)}_{\text {cellular death }},
$$

where $\eta_{1}, \eta_{2} \in \mathbb{R}_{+}$are constants representing the dependence of cellular proliferation and death on stress.

As a consequence of tumor incompressibility (assumption (vi)), we get from (7)

$$
\nabla \cdot \boldsymbol{v}=\alpha c\left(1+\eta_{1} \operatorname{tr}(\boldsymbol{\sigma})\right)-k\left(1-\eta_{2} \operatorname{tr}(\boldsymbol{\sigma})\right) .
$$

3.5. Nutrient concentration. The nutrient concentration variation is determined by nutrient diffusion through the boundary of the tumor and its consumption by tumor cells in the interior. From assumptions (iv) and (v), it is noticed that

$$
\frac{\partial c}{\partial t}+\boldsymbol{v} \cdot \nabla c=\underbrace{D_{c} \nabla^{2} c}_{\text {diffusion }}-\underbrace{A_{c} c \rho}_{\text {consumption }}
$$

where $c$ represents the nutrient concentration, $D_{c}$ is the diffusion rate (which is assumed to be constant), and $A_{c}$ is the nutrient consumption rate. Moreover, assuming that the nutrient concentration variation is much smaller than its diffusion and consumption, (9) can be written as

$$
D_{c} \nabla^{2} c=A_{c} c \rho .
$$

3.6. Nondimensionalization of the model. An important step in modeling is to work with nondimensional variables. With this purpose, we define the constants

$$
L \equiv \sqrt{\frac{D_{c}}{A_{c} \rho}}, \quad \tau \equiv \frac{1}{\alpha c_{b}}, \quad c_{b}, \quad \text { and } \quad \epsilon \equiv \frac{k}{\alpha c_{b}},
$$

which represent the length scale, time scale, constant nutrient concentration at the boundary, and ratio between the cellular death and cellular proliferation rates. We use asterisks to identify the nondimensional variables:

$$
r^{*}=\frac{r}{L}, \quad \sigma_{i j}^{*}=\frac{\sigma_{i j}}{E}, \quad p^{*}=\frac{p}{E}, \quad v^{*}=\frac{v}{\alpha c_{b} L}, \quad t^{*}=\frac{t}{\tau}, \quad \text { and } \quad c^{*}=\frac{c}{c_{b}} .
$$

These new variables are placed in (1), (2), (4), (6), (8), and (10). For simplicity of notation, the asterisks are removed.

The equation of nutrients, derived from (10), can be solved analytically. We thus get

$$
c(r, t)=\frac{R \sinh r}{r \sinh R}
$$


Then taking into account the radial symmetry $\boldsymbol{v}=\left(v_{r}, 0,0\right)$ and placing (11) in the equation resulting from the nondimensionalization of (8) leads to

$$
\begin{aligned}
\frac{\partial v_{r}}{\partial r}(r, t)=\frac{R \sinh (r)}{r \sinh (R)}\left[1+\eta_{1} E\left(3 \sigma_{r}(r, t)-2 \beta(r, t)\right)\right] \\
-\epsilon\left[1-\eta_{2} E\left(3 \sigma_{r}(r, t)-2 \beta(r, t)\right)\right]-2 \frac{v_{r}(r, t)}{r} .
\end{aligned}
$$

Now, in matrix form, (4) reads

$$
\begin{aligned}
\left(\begin{array}{ccc}
\frac{\partial v_{r}}{\partial r} & 0 & 0 \\
0 & \frac{v_{r}}{r} & 0 \\
0 & 0 & \frac{v_{r}}{r}
\end{array}\right)=\frac{1}{r^{2}} \frac{\partial}{\partial r}\left(r^{2} v_{r}\right)\left(\begin{array}{ccc}
\gamma_{r} & 0 & 0 \\
0 & \gamma_{\theta} & 0 \\
0 & 0 & \gamma_{\theta}
\end{array}\right) \\
+\frac{1}{2 E}\left(\frac{\partial}{\partial t}+v_{r} \frac{\partial}{\partial r}\right)\left(\begin{array}{ccc}
\sigma_{1} & 0 & 0 \\
0 & \sigma_{2} & 0 \\
0 & 0 & \sigma_{3}
\end{array}\right)
\end{aligned}
$$

with

$$
\begin{aligned}
& \sigma_{1}=2 \sigma_{r}-\sigma_{\theta}-\sigma_{\phi}, \\
& \sigma_{2}=2 \sigma_{\theta}-\sigma_{r}-\sigma_{\phi}, \\
& \sigma_{3}=2 \sigma_{\phi}-\sigma_{r}-\sigma_{\theta},
\end{aligned}
$$

where $\sigma_{r}, \sigma_{\theta}$, and $\sigma_{\phi}$ are the diagonal components of the Cauchy stress tensor $\sigma$. In (13) the diagonal elements are the only nonzero elements because of the symmetry assumptions. If the second diagonal element is subtracted from the third in the matrix equation, then

$$
\left(\frac{\partial}{\partial t}+v_{r} \frac{\partial}{\partial r}\right)\left(\sigma_{\theta}-\sigma_{\phi}\right)=0
$$

suggesting $\sigma_{\theta}=\sigma_{\phi}$ because the material derivative of $\sigma_{\theta}-\sigma_{\phi}$ is zero. Substituting this result in the first diagonal element of (13), we have

$$
\left(\frac{\partial}{\partial t}+v_{r} \frac{\partial}{\partial r}\right)\left(\sigma_{r}-\sigma_{\theta}\right)=2\left(\gamma_{\theta} \frac{\partial v_{r}}{\partial r}-\gamma_{r} \frac{v_{r}}{r}\right)
$$

As in (4), the equilibrium (2) represents a system of partial differential equations, i.e., three partial differential equations for three unknown functions $\left(\sigma_{r}, \sigma_{\theta}\right.$, and $\left.\sigma_{\phi}\right)$, and the only nonzero equality is that corresponding to the radial direction, i.e.,

$$
\frac{\partial \sigma_{r}}{\partial r}+\frac{2\left(\sigma_{r}-\sigma_{\theta}\right)}{r}=0
$$


Finally, from (1), (12), (14), and (15), the model is given by the system of firstorder partial differential equations

$$
\begin{aligned}
\frac{d R}{d t}(t)=v_{r}(R, t) & \text { for } t \in \mathbb{R}_{+}^{*}, \\
\frac{\partial v_{r}}{\partial r}(r, t)=\frac{R \sinh (r)}{r \sinh (R)}\left[1+\eta_{1} E\left(3 \sigma_{r}(r, t)-2 \beta(r, t)\right)\right] & \\
-\epsilon\left[1-\eta_{2} E\left(3 \sigma_{r}(r, t)-2 \beta(r, t)\right)\right] & \text { for } t \in \mathbb{R}_{+}^{*} \text { and } r \in(0, R], \\
-2 \frac{v_{r}(r, t)}{r} & \text { for } t \in \mathbb{R}_{+}^{*} \text { and } r \in[0, R), \\
\frac{\partial \sigma_{r}}{\partial r}(r, t)=-\frac{2 \beta}{r} & \text { for } t \in \mathbb{R}_{+}^{*} \text { and } r \in(0, R),
\end{aligned}
$$

with

$$
\begin{aligned}
& \varpi(r, t)=2\left(\gamma_{\theta} \frac{\partial v_{r}}{\partial r}-\gamma_{r} \frac{v_{r}}{r}\right) \\
&=2 \gamma_{\theta}\left(\frac{R \sinh (r)}{r \sinh (R)}\left[1+\eta_{1} E\left(3 \sigma_{r}(r, t)-2 \beta(r, t)\right)\right]\right. \\
&\left.\quad-\epsilon\left[1-\eta_{2} E\left(3 \sigma_{r}(r, t)-2 \beta(r, t)\right)\right]\right)-2 \frac{v_{r}(r, t)}{r},
\end{aligned}
$$

subject to the initial and boundary conditions

$$
\begin{aligned}
R(0) & =R_{0}, \\
\sigma_{r}(r, 0) & =0, \\
\beta(r, 0) & =0, \\
v(0, t) & =0,
\end{aligned}
$$

where $\beta=\sigma_{r}-\sigma_{\theta}$. Two conditions are still needed for $\beta$ and $\sigma_{r}$.

(i) Condition for $\beta$ at $r=0$. Since $v_{r}=0$ at $r=0$ and the derivatives of $\beta$ are bounded (because $\beta$ is assumed $C^{1}$ in $[0, R]$ with respect to $r$ ), then

$$
\frac{\partial \beta}{\partial t}(0, t)=\varpi(0, t) .
$$

Therefore, the first boundary condition for $\beta$ is

$$
\begin{aligned}
\frac{\partial \beta}{\partial t}(0, t)=\left(2 \gamma_{\theta}-1\right)\left(\frac{R}{\sinh (R)}[\right. & \left.1-\eta_{1} E\left(3 \sigma_{r}(0, t)-2 \beta(0, t)\right)\right] \\
& \left.-\epsilon\left[1+\eta_{2} E\left(3 \sigma_{r}(0, t)-2 \beta(0, t)\right)\right]\right)+\frac{\partial v_{r}}{\partial r}(0, t) .
\end{aligned}
$$


(ii) Condition for $\sigma_{r}$. This condition may be determined if the constitutive equation (6) of the external medium is used assuming the continuity of the stresses at the tumor boundary. Because of the spherical tumor symmetry from hypothesis (iii) and the incompressibility of the external medium (hypothesis (viii)),

$$
\operatorname{tr}(\boldsymbol{e})=\frac{1}{r^{2}} \frac{\partial}{\partial r}\left(r^{2} u_{r}\right)=0 .
$$

Substituting the solution of the above equation in (6), we have

$$
\left.\sigma_{r}\right|_{r=R}=-\frac{4\left(R-R_{0}\right)}{3 R} \text {. }
$$

\section{Cellular automata model definition}

A cellular automaton based on a linear elasticity tumor model [Ramírez-Torres et al. 2016] is defined. The model described in [Ramírez-Torres et al. 2016] and summarized in the previous section is taken as an example of a system of differential equations (DE) that represents tissue growth, i.e., tumor growth in this case.

The present continuum-mechanical model considers the tumor as a solid in the three-dimensional space, and the forces on it are considered acting per volume unit. As a result of the spherical symmetry hypothesis, the body deformation is the same in two of the three principal directions and no shear deformations are accounted for. Moreover, from the symmetry condition, all the fields depend only on time $t$ and on one spatial variable $r$. The three principal stresses are $\sigma_{r}, \sigma_{\theta}$, and $\sigma_{\phi}$, with (as shown in the text after (13)) $\sigma_{\theta}=\sigma_{\phi}$. In this sense, we only need to find $\sigma_{r}$ and $\sigma_{\theta}$, given that the stress in the direction orthogonal to the plane where they are contained, corresponding to the third principal stress, is equal to $\sigma_{\theta}$ and therefore not necessarily zero. Hence, the theoretical model is addressed as neither a plane strain nor as a plane stress problem [Sokolnikoff 1956].

On the other hand, for representing the cellular automaton model, a cross section passing through the origin of the tumor in the three-dimensional model is taken. It does not matter which cross section because, given the spherical symmetry of the continuous theoretical model, all the cross sections are equivalent. Therefore, the cellular automaton is constructed in a two-dimensional setting, although the model is easily extended to three dimensions. Indeed, the rules of the model are given for any spatial dimension. Hence, the stresses and deformations in the cellular automaton model are the same stresses and deformations which are completely contained in a cross section of the continuum-mechanical model.

The transformation of the deterministic DE linear elasticity tumor model into a cellular automaton is required. The neighborhood structure and the rules for a probabilistic CA are deduced from continuous differential equations. The CA solution presented in this work can be extended to other similar continuous DE models. 
4.1. General model definition. In the present study, a simple two-state cellular automaton is considered, where a 0 -state represents a normal cell and a 1-state exemplifies a tumoral cell.

The automaton is considered infinite because it is defined in a borderless square lattice in which there exists some central cell with coordinates $(0,0)$. The initial state assigned to each cell is 0 , except for a finite number of them. Practical implementations of this model must maintain a finite matrix representation of the relevant part of the lattice and expand it on demand.

The neighborhood structure and a rule set that describes the continuous model behavior are explained in the following.

4.2. Neighborhood structure selection. There is an equivalence (1) between the tumor radius growth speed and the tumor cell displacement speed in the continuous model described in [Ramírez-Torres et al. 2016]:

$$
\frac{d R}{d t}=v(R, t)
$$

The speed $v$ could be used in the definition of the transition rules of the CA because the "displacement speed" oriented outside the tumor is closely related to the tumor cell propagation. In addition, we know that $v$ does not depend explicitly on the spatial coordinates of a point but instead on the radial one. This fact implies that the "influence" of a tumor is equally distributed in all directions from the center of the model.

In order to select the appropriate neighborhood structure, we should take into account this property of the tumor growth speed. Since all the discrete time steps are equal, the increments of the tumor cell coordinates should also be equal, for the same moment of time, in all directions. In the CA, the increment of the tumor cell coordinate is described as the propagation of some tumor cell into another normal cell.

Therefore, the influence zone (neighbor) of any cell $c$ must have cells with the same distance to $c$. We can choose some constant distance $q$, and for any cell $c_{0}$, the neighborhood is defined as

$$
\left\{c \in C: d\left(c_{0}, c\right)=q\right\},
$$

where $d\left(c_{1}, c_{2}\right)$ is the common Euclidean distance in the unit square lattice.

We choose $q=1$, which generates a well known von Neumann neighborhood [Deutsch and Dormann 2005]. It is possible to choose other values of $q$, such as $q=\sqrt{2}$ or $q=2$, generating other unexplored neighborhoods.

4.3. Rule inference. The main principle used in the area of rule inference from continuous models [Guinot 2002] consists of creating a stochastic rule with the 
structure

$$
s_{c}(t+\Delta t)= \begin{cases}s_{c}(t)+1, & X \leq g\left(s_{c}(t), N(c), \Delta t\right), \\ s_{c}(t), & X>g\left(s_{c}(t), N(c), \Delta t\right),\end{cases}
$$

where $X$ is a random variable with uniform distribution in $(0,1)$ and $\Delta t$ is the length of the time step. The hardest part of rule inference is precisely the correct and meaningful definition of $g$. Actually, function $g$ expresses the idea of the "speed" of growing in some place of the lattice where conditions around the cell are expressed by the state of the cell itself $s_{c}(t)$ and by its neighborhood $N(c)$.

From the continuous model, the differential equation (1) shows the relationship between the tumor radius growth speed and the radius itself:

$$
\frac{d R}{d t}=v(R, t)
$$

Initially, we assume that $v(R, t) \geq 0$, i.e., the size of the tumor is nondecreasing. We interpret $\frac{d R}{d t}$ as the average number of new tumor cells created from one tumor cell per unit of time. If $\frac{d R}{d t}<1$, this speed is seen as the probability of appearance of a new tumor cell during the unit time step, i.e., function $g$. Otherwise, some normalization process over $\frac{d R}{d t}$ becomes necessary.

In the case when $\eta_{1}=\eta_{2}=0$ (stress dependence is not considered), a closedform expression for $v$ can be obtained from (16)-(19):

$$
\frac{d R}{d t}=\frac{\cosh R}{\sinh R}-\frac{1}{R}-\frac{\epsilon R}{3},
$$

where $\epsilon$ is a constant. The fact that $v(R, t)=v(R)$, depending only on the maximum tumor radius, is particularly important for the rule definition process because cellular automaton inherently does not have any notion of time: the next state depends only on the current state.

However, if $\eta_{1} \neq 0$ or $\eta_{2} \neq 0$, then the stress influence is taken into account and no closed-form expression can be obtained for $v$. In this case, $v(R, t)$ is approximated using numerical methods, like Euler's method or the Lax-Wendroff scheme from finite differences.

4.3.1. Case without stress dependence. If $\eta_{1}=\eta_{2}=0$, stresses are not taken into account, and a closed-form expression (21) for $v$ is obtained from the differential equations (16)-(19). The properties of this equation are analyzed in order to appropriately define $g$.

Stability analysis. Equation (21) has the form $R_{t}^{\prime}=v(R)$, where $R_{t}^{\prime}$ is the derivative of the radius function with respect to time. In the classical numerical methods, we can use the fact that, for a small $h$,

$$
R_{t}^{\prime} \approx \frac{R_{t+1}-R_{t}}{h} \quad \text { and } \quad R_{t+1} \approx R_{t}+h v(R) .
$$




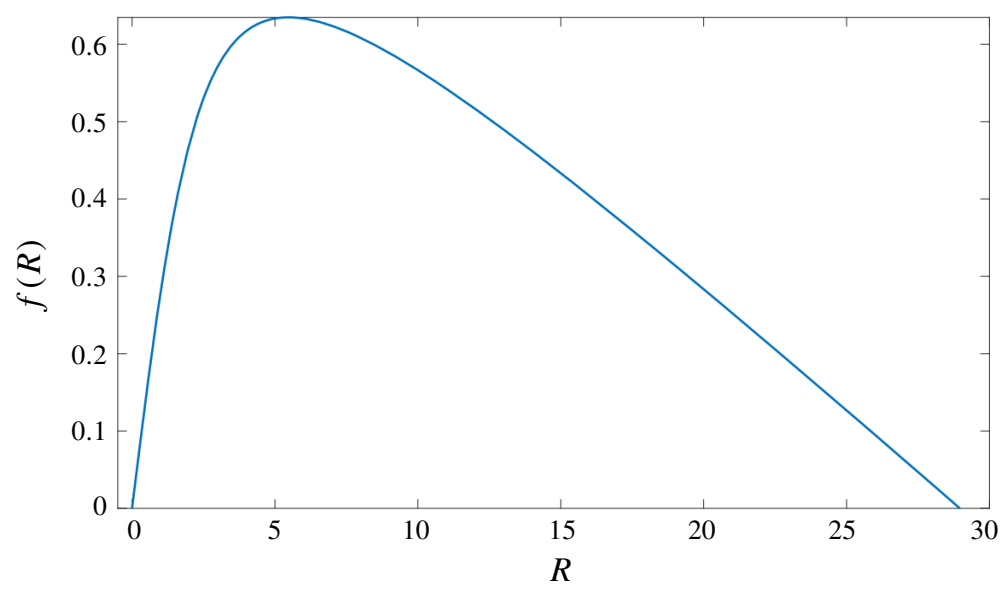

Figure 1. Case without stress dependence, stability analysis: increment function $(\cosh R) /(\sinh R)-1 / R-\epsilon R / 3$ for $\epsilon=0.1$.

It is clear that, for a discrete time step $h, v(R)$ represents the increment in the tumor radius from a time moment to the next. In terms of cellular automata, it represents the magnitude of propagation of a tumoral cell $c$ into its normal neighbor. However, since CA are discrete computational systems in space, the tumoral cell cannot be expanded in a fractional amount of space.

In Figure 1, the increment $v(R)$ reaches a local maximum close to $v=0.63$ and has a root close to $R=29$. The tumor radius starts its growth at $R=R_{0} \geq 0$. Since the increment is always positive, it grows asymptotically to the root value, where the increment is null.

We analyze the rule inference process, where the function $g$ is appropriately defined. In rule (20), $g$ indicates the probability of expansion of a tumoral cell into a normal neighbor. In this sense, the desired expansion probability is exactly the magnitude of propagation of the tumor cell. Since the increment $v(R) \in[0,1]$ is bounded, it can be used as the probability $g$, simply setting $g=v$. If $v(R) \notin[0,1]$, it would have been necessary to perform some normalization process over $v(R)$.

To summarize, if the stress dependence is not considered, the following stochastic rule emerges:

$$
s_{c}(t+\Delta t)= \begin{cases}s_{c}(t)+1, & X \leq v(r), \\ s_{c}(t), & X>v(r),\end{cases}
$$

where $X$ is a random variable with uniform distribution in $(0,1)$,

$$
v(r)=\frac{\cosh r}{\sinh r}-\frac{1}{r}-\frac{\epsilon r}{3},
$$


and $r=\sqrt{i^{2}+j^{2}}$ is the radial coordinate of a cell. Although the angular coordinate is not present in this rule, this does not mean that the model is symmetric in all polar directions. The asymmetry is present indirectly through random variable $X$ whose realizations must be generated at every time step and for each cell [Guinot 2002], leading to an irregular shape.

4.3.2. Case with stress dependence. The assumption that $\eta_{1} \neq 0$ or $\eta_{2} \neq 0$ leads to a scenario where the stresses' influence is considered. In particular, no closedform expression can be obtained for $v$ in this case, making the use of numerical methods unavoidable. Therefore, $v$ is approximated using Euler's method or the Lax-Wendroff scheme from finite differences [Ramírez-Torres et al. 2016]. More precisely, numerical approximations of $R(t)$ and $v(R, t)$ are obtained. This means that pairs $\left(R_{i}, v\left(R_{i}\right)\right)$ are computed for each moment in time $t_{i}$, where

- $R_{i}$ is the tumor radius at $t_{i}$ and

- $v\left(R_{i}\right)$ is the tumor growth speed at any point of the tumor border at $t_{i}$.

There is no guarantee of the existence of a functional dependency between $v$ and $R$, as in (21). In fact, there are cases where for the same value of $R$, more values of $v$ are obtained. One example is shown in Figure 2, where no $v(R)$ can be defined because $R(t)$ is not injective, with two distinct growth speeds at the same radius, at two distinct moments of time.

We first analyze the case where the function $v(R)$ is approximately definable. Next, we examine, the case where the function $v(R)$ cannot be defined.

(i) Definable $v(R)$. If $R(t)$ is a strictly increasing function, then it is also injective and an ordered sequence of pairs $\left(R_{i}, v\left(R_{i}\right)\right)$ is obtained, where all radius values

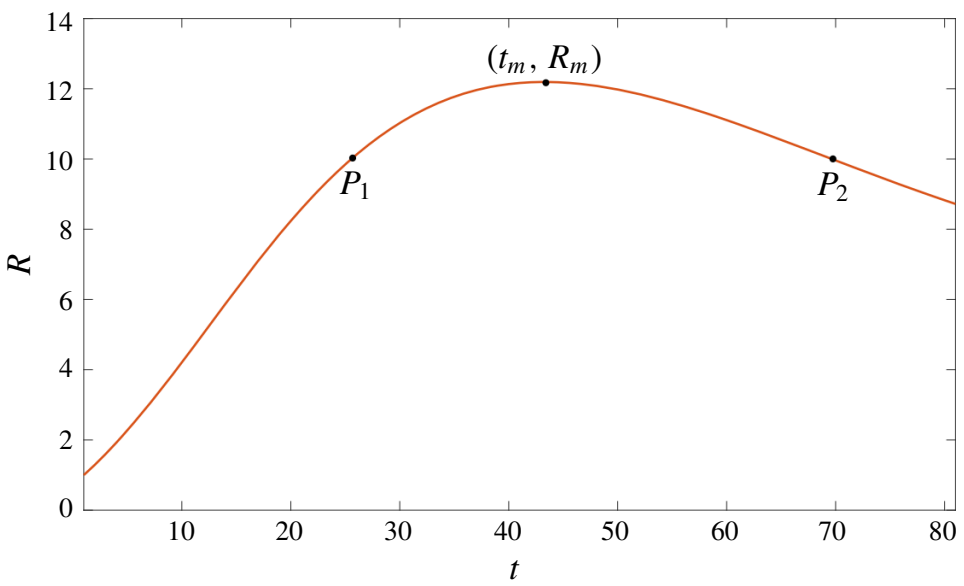

Figure 2. Tumor radius growth in time, theoretical model, strict extremum case. 
are different. In this case, function $v(R)$ is approximately defined in the following way. To evaluate $v(R)$ for some $R=R_{j}$, the speed value that corresponds to the closest to $R_{j}$ among all the radius values in the sequence is selected.

The smaller the time step becomes, the better the approximation is, since there is a higher density of available $R$ values.

Given this definition of $v(R)$, the stochastic rule for the CA is defined in a similar way as in the case without stress dependence (22):

$$
s_{c}(t+\Delta t)= \begin{cases}s_{c}(t)+1, & X \leq v\left(R_{i}\right), \\ s_{c}(t), & X>v\left(R_{i}\right) .\end{cases}
$$

Here, $v\left(R_{i}\right)$ is the evaluation of the radius $R_{i}$ that is closest to the radial coordinate of the cell value, from the available radius-speed evaluations.

The sequence of pairs $\left(R_{i}, v\left(R_{i}\right)\right)$ is called from now on the guide set and represents an approximation to the actual $v$ function. It is used as a guide during the CA evolution process.

(ii) Undefinable $v(R)$. If $R(t)$ is not a strictly increasing (or strictly decreasing ${ }^{1}$ ) function, the injectivity of $R(t)$ cannot be guaranteed and no simple functional dependency between $v$ and $R$ can be defined.

In particular, the special case where $R(t)$ has a strict extremum is considered. In Figure 2 an example is shown. The existence of some pair $\left(R_{m}, v\left(R_{m}\right)\right)$ is assumed, where $R_{m}$ is a maximum over the $R$ values available in the guide set.

The impossibility of defining a single CA that represents the continuous model behavior is explained below. Choosing two time moments with the same tumor radius (points $P_{1}$ and $P_{2}$ in Figure 2), similar CA configurations are expected in both cases. Then since the cellular automaton is a memoryless system (no time variable or history can be present), it is not possible to distinguish between both states, in order to determine when it should grow or when a reduction should take place, since the next state of any CA only depends on its previous state.

In response to the need of having the tumor growth process representation in this case, a solution based on the use of two cellular automata is proposed. Having a sequence of the $\left(R_{i}, v\left(R_{i}\right)\right)$ pairs ordered by $i$, the sequence is divided into two subsequences. The first is the sequence $s_{1}$ that starts with $\left(R_{0}, v\left(R_{0}\right)\right)$ and ends with $\left(R_{m}, v\left(R_{m}\right)\right)$; the second sequence $s_{2}$ contains the rest of the radius-speed pairs up to the last (see Figure 2).

It was shown that a guide set like $s_{1}$ can define an approximation to the positive $v$ function needed to define the rule (23). Another rule that reflects tumor reduction

${ }^{1}$ The assumption that $v(R, t) \geq 0$ is relaxed here; i.e., the tumor may decrease with negative values of $v$. 
is then required. In this sense, the following rule structure is proposed:

$$
s_{c}(t+\Delta t)= \begin{cases}s_{c}(t)-1, & X \leq\left|v\left(R_{i}\right)\right|, \\ s_{c}(t), & X>\left|v\left(R_{i}\right)\right| .\end{cases}
$$

This rule is applied to the tumoral cells with normal neighbors, where $X$ is a random variable with uniform distribution in $(0,1)$ and $v\left(R_{i}\right)$ is the evaluation of the radius $R_{i}$ from $s_{2}$ that is closest to the radial coordinate of the cell $r$.

Finally, the complete model is defined as an ordered pair $\left(A_{1}, A_{2}\right)$, where:

- $A_{1}$ corresponds to the first of the two automata, with stochastic rule (23), and guide set $s_{1}$ and

- $A_{2}$ corresponds to the second automaton, with its initial state being the final state of $A_{1}$, stochastic rule (24), and guide set $s_{2}$.

4.3.3. Rule definition summary. To summarize, a new stochastic model of tumor growth obtained from a differential equation system is provided. A random variable $X$ with uniform distribution in $(0,1)$ is considered. If a closed-form expression of the growth speed $v$ in the tumor border can be found, then the following rule is defined:

$$
s_{c}(t+\Delta t)= \begin{cases}s_{c}(t)+1, & X \leq v(r) \\ s_{c}(t), & X>v(r)\end{cases}
$$

where $v(r)=(\cosh r) /(\sinh r)-1 / r-\epsilon r / 3$ and $r=\sqrt{i^{2}+j^{2}}$ is the radial coordinate of the cell $c$ in the lattice.

If a closed-form expression for $v$ cannot be found, then a guide set is defined as a collection of pairs $\left(R_{i}, v\left(R_{i}\right)\right)$ ordered by the time moment at which the approximation is made. If $v \geq 0$, then the following first CA rule can be applied to the normal cells with at least one tumoral cell in its neighborhood:

$$
s_{c}(t+\Delta t)= \begin{cases}s_{c}(t)+1, & X \leq v\left(R_{i}\right), \\ s_{c}(t), & X>v\left(R_{i}\right),\end{cases}
$$

where $v\left(R_{i}\right)$ is the evaluation of $v$ at the radius $R_{i}$ from the guide set that is closest to the radial coordinate of the cell $r$. We remark that this rule is applied to the cells with tumoral neighbors and, in particular, to the cells on the border of the tumor, where $r \approx R$.

If $v<0$, then a second CA rule is applied to the tumoral cells with normal neighbors:

$$
s_{c}(t+\Delta t)= \begin{cases}s_{c}(t)-1, & X \leq\left|v\left(R_{i}\right)\right|, \\ s_{c}(t), & X>\left|v\left(R_{i}\right)\right|,\end{cases}
$$

where, once again, $v\left(R_{i}\right)$ is the evaluation of $v$ from the guide set at the closest point to the radial coordinate of the cell $r$. 


\section{Results}

One important issue in any model is the validation of its consistency with the original phenomenon or another reference model. In this case, a comparison between the behavior of the discrete CA model described above and the continuous model is provided.

In this section, experimental results are discussed for all three cases of tumor growth: without stress dependence, with stress dependence and strictly increasing tumor radius, and with stress dependence and a tumor radius that has a strict extremum.

The CA model described above was implemented using the $\mathrm{C} \#$ programming language on an Intel Core i 7 machine, with a $2.0 \mathrm{GHz}$ processor and $16 \mathrm{~GB}$ of random-access memory. All executions were very fast, taking a few seconds of running time.

Common values of the parameters $\epsilon=0.1, R_{0}=1$, and $E=64.0439$ were fixed in all computations. An important clarification is that the tumor radius value in the cellular automaton is computed as the average between the tumoral border cell distances to the center of the tumor.

5.1. Tumor growth without stress dependence. In the case where $\eta_{1}=\eta_{2}=0$, the stresses are not taken into account in the mathematical model. Then the cellular automaton with rule (22) behavior is compared with the continuous differential equation model. The results are shown in Figure 3.

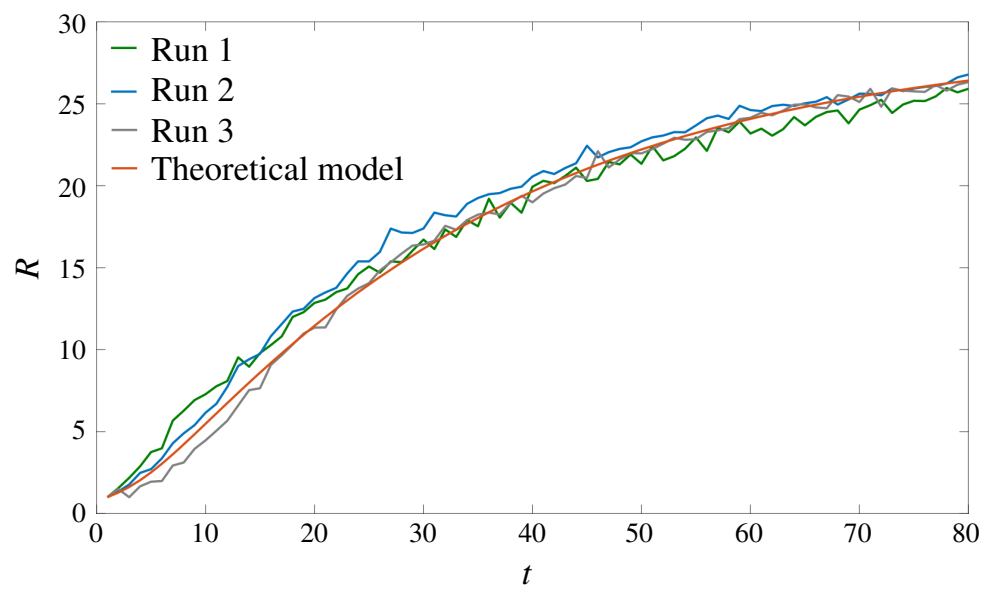

Figure 3. Graphic illustrating the evolution of the tumor radius with time, case without stress dependence. Comparison between several runs of the discrete CA model and the continuous (red line) model. The continuous model is obtained from (21), as a particular case of the model defined by (16)-(19). 

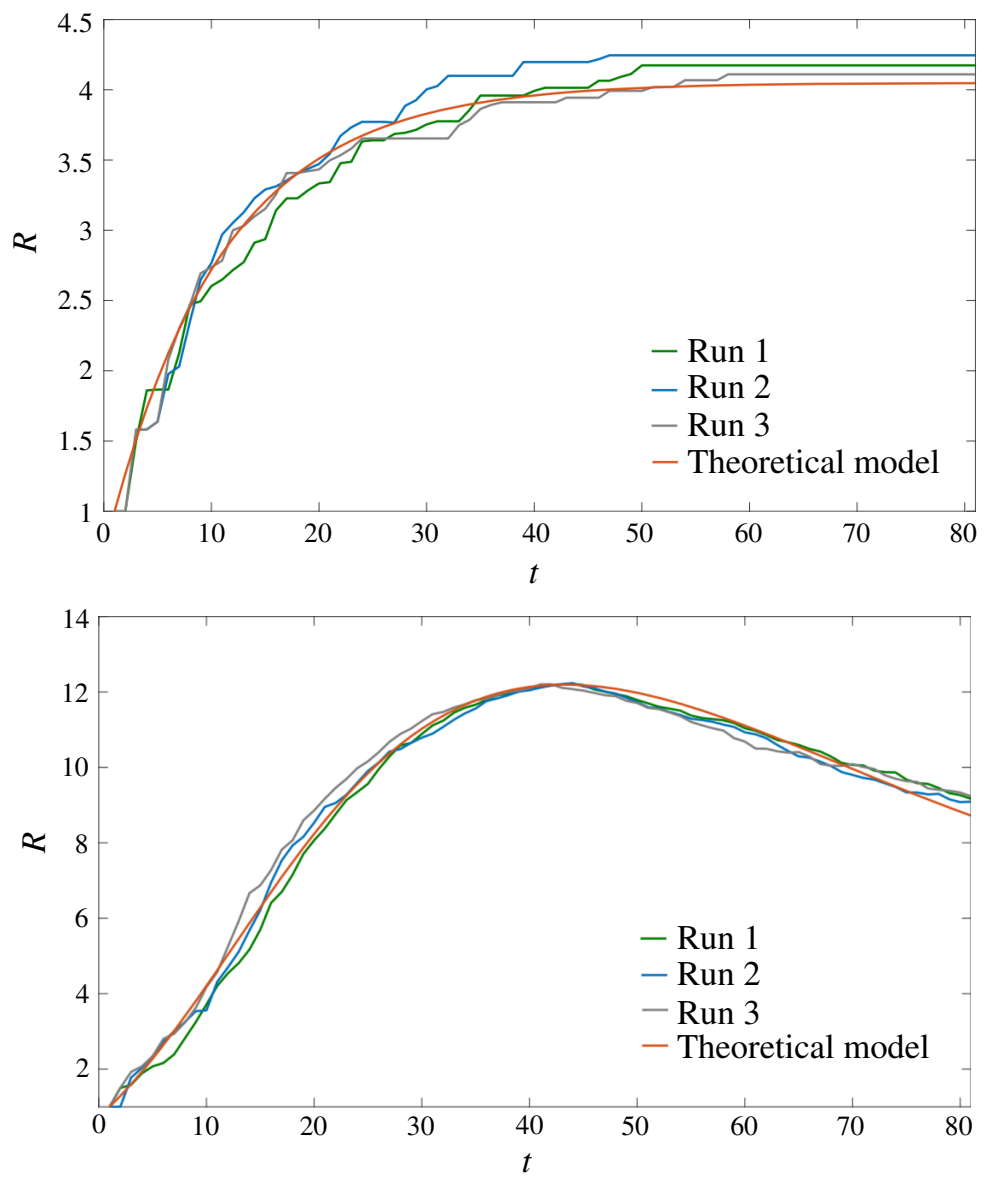

Figure 4. Graphics illustrating the evolution of the tumor radius with time, cases with stress dependence when the tumor radius is strictly increasing (top) and when the tumor radius has a strict extremum (bottom). Comparison between several runs of the discrete CA model and the continuous (red line) model.

The radius-time dependence graphics are similar and close to each other. A small difference between them is due to the dissimilar nature of the models.

5.2. Tumor growth with stress dependence. Similar experiments are performed in the case of stress-dependent tumor growth. Results are shown in Figure 4.

The case where the tumor radius is a strictly increasing function is obtained with $\gamma_{\theta}=\frac{1}{3}$ and $\eta_{1}=0.004$ (Figure 4, top). A cellular automaton with rule (23) is used.

The case where the tumor radius has a strict extremum is obtained with $\gamma_{\theta}=\frac{1}{10}$ and $\eta_{1}=0.002$ (Figure 4, bottom). In this case, two cellular automata with rules (23) and (24), respectively, are used. 

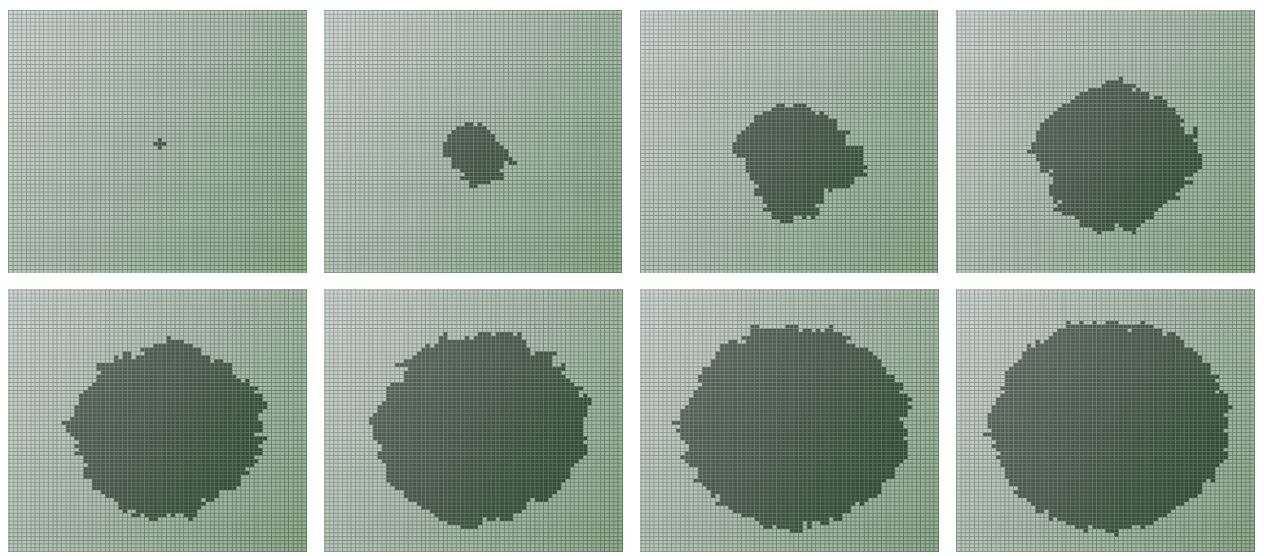

Figure 5. Visualizations of the CA tumor growth for time moments $0,10,20,30,40,50,60$, and 70, respectively.

In both cases, close radius-time dependence graphics are observed. Stabilization of tumor radius growth is detected in the strictly increasing function case (Figure 4, top) in all runs. An excellent degree of proximity is achieved in the strict extremum case (Figure 4, bottom) with two cellular automata.

5.3. Tumor growth visualization in time. An example of the tumor growth visualization is shown in Figure 5. It corresponds to the case without stress dependence described in Section 5.1.

In particular, we notice an exponential, fast growth at the beginning, followed by an asymptotic behavior when the radius of the tumor stabilizes around a constant value. Further visualizations are not shown due to their similarity with those corresponding to $t=60$ and $t=70$. There is almost no change when the running time is increased because of the strong convergence of the tumor radius in the CA model.

This corresponds to the stability results obtained in Section 4.3.1 and shown in Figure 1, where the radius increment function has a zero at a radius value slightly below 29.

5.4. Dispersion of the executions. Finally, the dispersion of computational executions is evaluated. As an example, the case without stress dependence is considered, whose visualizations were shown in the previous section. Firstly, the average execution is computed as a sequence of averages of tumor radius values obtained from $100 \mathrm{CA}$ model runs, for each time step. The resulting graphic is shown in Figure 6. It compares the CA average execution with the continuous model: both functions are very close to each other.

Figure 7 displays the coefficient of variation. Most of the chart is below the 


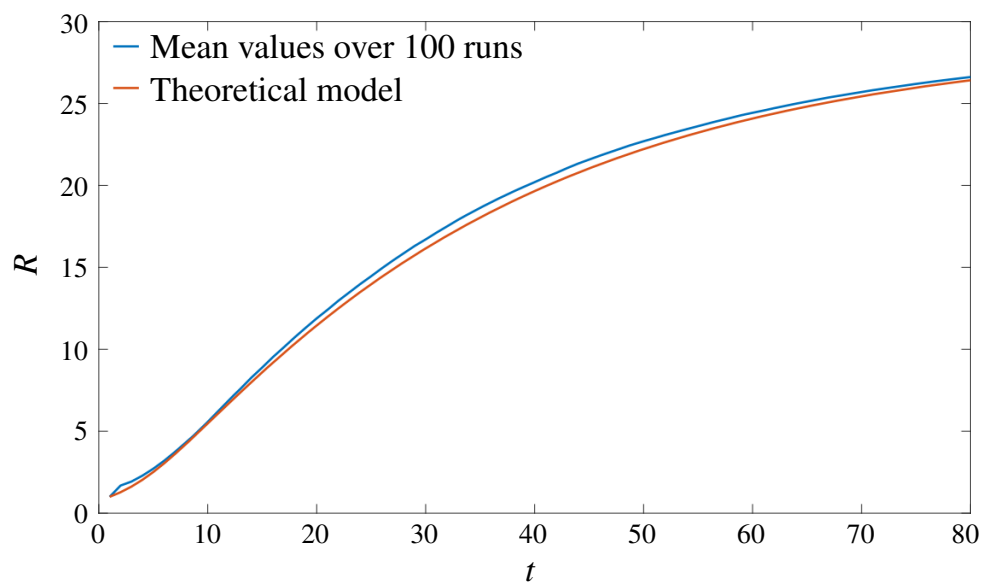

Figure 6. The blue line shows average values of the tumor radius variable over $100 \mathrm{CA}$ model runs. Each point is obtained as the average of 100 tumor radius values obtained at that moment of time by 100 independent executions of the cellular automaton model. The red line shows the continuous model obtained from (21) as a particular case of the model defined by (16)-(19).

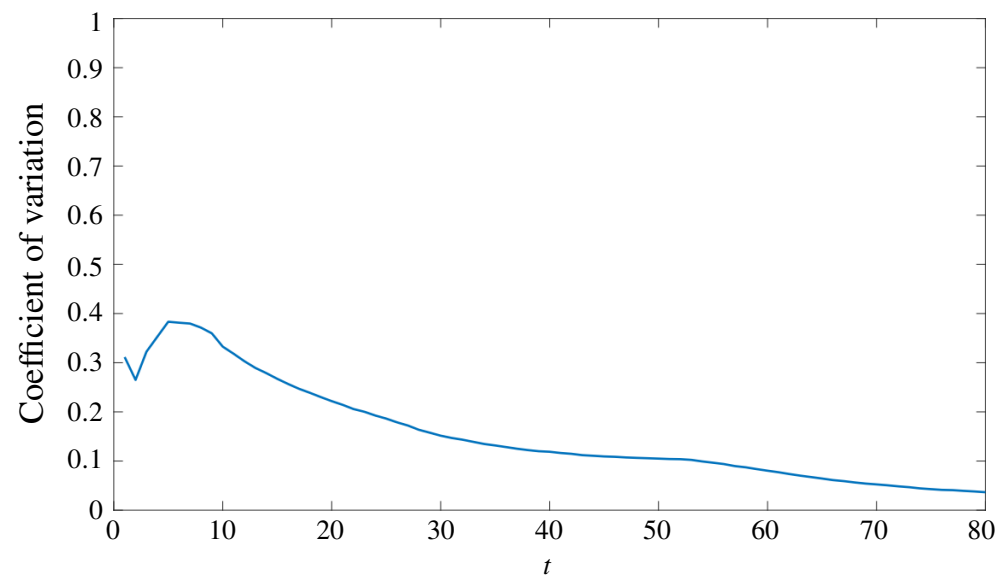

Figure 7. Values (in percent) of the coefficient of variation of the tumor radius variable for the CA model. Each point is obtained from 100 tumor radius values reached at that moment of time by 100 independent executions of the CA model.

$20 \%$ line, indicating small variations of the tumor radius. Larger variations are observed only near the start of the executions. This shows a stable behavior of the computational model beyond its stochastic nature. 


\section{Conclusions}

A new stochastic cellular automata model for the process of tumor growth is proposed. From a linear elasticity deterministic continuous tumor model described in [Ramírez-Torres et al. 2016], a neighborhood structure and stochastic automata rules are deduced. The results allow one to visualize the growth process described in the continuous model in a more realistic manner since tumors are neither regular nor perfectly circular. Moreover, stress influence in the growing of the tumor is taken into account at the time when the rules of the CA model are derived. The differences in the tumor radius between the two models are small and are actually due to their different nature. Validation tests confirmed that the CA model accurately captures the hypothesis of the described phenomena. The methodology exposed in this work can be applied to other continuous DE models in order to represent the growth processes in a nonidealized and nondeterministic way.

\section{Acknowledgments}

Ruben Interian has been sponsored by a CAPES scholarship. Ariel Ramírez-Torres thanks the postdoctoral funding of the Program of Postdoctoral Scholarships DGAPA from UNAM and professors Federico J. Sabina and Catherine García-Reimbert for their support. Work of Celso C. Ribeiro was partially supported by CNPq research grant 459575/2014-7 and by FAPERJ research grant E-26/201.198/2014. Aura Conci is partially supported by FAPERJ project SIADE2 and by CNPq project number 303240/2015-6.

\section{References}

[Anderson et al. 2009] A. R. A. Anderson, K. A. Rejniak, P. Gerlee, and V. Quaranta, "Microenvironment driven invasion: a multiscale multimodel investigation", J. Math. Biol. 58:4-5 (2009), $579-624$.

[Berto and Tagliabue 2012] F. Berto and J. Tagliabue, "Cellular automata", electronic reference, 2012, Available at http://plato.stanford.edu/entries/cellular-automata/.

[Boondirek et al. 2010] A. Boondirek, W. Triampo, and N. Nuttavut, "A review of cellular automata models of tumor growth", Int. Math. Forum 5:61 (2010), 3023-3029.

[Deutsch and Dormann 2005] A. Deutsch and S. Dormann, Cellular automaton modeling of biological pattern formation: characterization, applications, and analysis, Birkhäuser, 2005.

[Dormann and Deutsch 2002] S. Dormann and A. Deutsch, "Modeling of self-organized avascular tumor growth with a hybrid cellular automaton”, In Silico Biol. 2:3 (2002), 393-406.

[Guinot 2002] V. Guinot, "Modelling using stochastic, finite state cellular automata: rule inference from continuum models", Appl. Math. Model. 26:6 (2002), 701-714.

[Hoehme and Drasdo 2010] S. Hoehme and D. Drasdo, "A cell-based simulation software for multicellular systems”, Bioinformatics 26:20 (2010), 2641-2642. 
[Hu and Ruan 2003] R. Hu and X. Ruan, "A simple cellular automaton model for tumor-immunity system”, pp. 1031-1035 in IEEE International Conference on Robotics, Intelligent Systems and Signal Processing (Changsha, China, 2003), vol. 2, Inst. Electrical and Electronics Engrs., 2003.

[Kansal et al. 2000a] A. Kansal, S. Torquato, E. A. Chiocca, and T. S. Deisboeck, "Emergence of a subpopulation in a computational model of tumor growth", J. Theor. Biol. 207:3 (2000), 431-441.

[Kansal et al. 2000b] A. Kansal, S. Torquato, G. R. Harsh, IV, E. A. Chiocca, and T. S. Deisboeck, "Simulated brain tumor growth dynamics using a three-dimentional cellular automaton", J. Theor. Biol. 203:4 (2000), 367-382.

[Merks and Glazier 2005] R. M. H. Merks and J. A. Glazier, "A cell-centered approach to developmental biology", Physica A 352:1 (2005), 113-130.

[Ngwa and Agyingi 2012] M. Ngwa and E. Agyingi, "Effect of an external medium on tumor growthinduced stress", IAENG Int. J. Appl. Math. 42:4 (2012), 229-236.

[Ramírez-Torres et al. 2016] A. Ramírez-Torres, F. Valdés-Ravelo, R. Rodríguez-Ramos, J. BravoCastillero, R. Guinovart-Díaz, and F. J. Sabina, "Modeling avascular tumor growth via linear elasticity", pp. 1739-1740 in Contributions to the foundations of multidisciplinary research in mechanics (Montreal, 2016), vol. 3, edited by J. M. Floryan, Proc. Int. Congress Theoretical and Applied Mechanics 24, Int. Union Theoretical Applied Mechanics, 2016.

[Rejniak and Anderson 2011] K. A. Rejniak and A. R. A. Anderson, "Hybrid models of tumor growth", Wiley Interdiscip. Rev. Syst. Biol. Med. 3:1 (2011), 115-125.

[Rejniak and McCawley 2010] K. A. Rejniak and L. J. McCawley, "Current trends in mathematical modeling of tumor-microenvironment interactions: a survey of tools and applications", Exp. Biol. Med. 235:4 (2010), 411-423.

[Sokolnikoff 1956] I. S. Sokolnikoff, Mathematical theory of elasticity, 2nd ed., McGraw-Hill, 1956.

Received 4 Dec 2016. Revised 23 Apr 2017. Accepted 6 Jun 2017.

RUBEN INTERIAN: rubenus@yandex.ru

Institute of Computing, Fluminense Federal University, Niterói, Brazil

REINALDO RODRÍGUEZ-RAMOS: rerora2006@gmail.com

Mathematics and Computer Science Faculty, Havana University, Havana, Cuba

FERnANDo VALDÉS-RAVELO: fernandov@cim.sld.cu

Molecular Immunology Center, Havana, Cuba

ARIEL RAMÍREZ-TORRES: arielrt02@gmail.com

Institute for Research in Applied Mathematics and Systems, National Autonomous University of Mexico, Mexico City, Mexico

Celso C. Ribeiro: celso@ic.uff.br

Institute of Computing, Fluminense Federal University, Niterói, Brazil

AURA CONCI: conci.aura@gmail.com

Institute of Computing, Fluminense Federal University, Niterói, Brazil 



\title{
A VARIATIONAL FORMULATION FOR FUZZY ANALYSIS IN CONTINUUM MECHANICS
}

\author{
ROLF MAHNKEN
}

\begin{abstract}
In order to improve the credibility of modern simulation tools, uncertainties of different kinds have to be considered. This work is focused on epistemic uncertainties in the framework of continuum mechanics, which are taken into account by fuzzy analysis. The underlying min-max optimization problem of the extension principle is approximated by $\alpha$-discretization, resulting in a separation of minimum and maximum problems. To become more universal, socalled quantities of interest are employed, which allow a general formulation for the target problem of interest. In this way, the relation to parameter identification problems based on least-squares functions is highlighted. The solutions of the related optimization problems with simple constraints are obtained with a gradient-based scheme, which is derived from a sensitvity analysis for the target problem by means of a variational formulation. Two numerical examples for the fuzzy analysis of material parameters are concerned with a necking problem at large strain elastoplasticity and a perforated strip at large strain hyperelasticity to demonstrate the versatility of the proposed variational formulation.
\end{abstract}

\section{Introduction}

The reliability assessment of components and engineering structures in today's industry is commonly performed by extensive use of numerical simulations. In order to improve its credibility uncertainties have to be taken into account, where two categories are distinguished, aleatoric and epistemic; see, e.g., [Sullivan 2015]. Aleatoric (or irreducible, stochastic) uncertainties refer to variability as a consequence of, e.g., fluctuations through time, variation across space, or manufacturing differences. In principle, they cannot be reduced by empirical effort; however, provided sufficient information is available they can be taken into account

\section{Communicated by Paul Steinmann.}

This work is based on investigations of SPP 1886: "Polymorphe Unschärfemodellierungen für den numerischen Entwurf von Strukturen", which is kindly supported by the Deutsche Forschungsgemeinschaft (DFG).

MSC2010: 74-XX.

Keywords: fuzzy analysis, $\alpha$-level optimization, quantities of interest, optimization with simple constraints, large strain elasticity, large strain elastoplasticity. 
with probabilistic concepts. Epistemic (reducible, subjective) uncertainties refer to subjectivity as a consequence of, e.g., incomplete scientific understanding or lack of measurements, which indicate a possible value range rather than a probability function. Contrary to aleatoric uncertainties, in principle they can be reduced by empirical effort, e.g., investing more in measurement.

Methodologies for the modeling of epistemic uncertainties are, e.g., interval analysis and, increasingly applied over the last few years, fuzzy analysis. Though the development of fuzzy arithmetic was started in the 1960s by Zadeh [1965], its application in solid mechanics is a rather new research area. Fuzzy analysis allows one to identify sensitivities of the response of a structure with respect to the magnitude of imprecision of the input data. The underlying axiomatic formalism is provided by the extension principle [Möller and Beer 2004]. It specifies the mapping of the fuzzy input set into a new fundamental set and results in solution of a maximum-minimum problem. In order to alleviate the complexity of this demanding task, $\alpha$-level optimization is employed. Here the membership input functions are discretized and maximum-minimum problems on each level eventually render a discretized solution for the membership output functions. The higher the chosen number of $\alpha$-levels, the greater the accuracy of the membership output of the response is.

Both the extension principle as well as $\alpha$-discretization may result in significant numerical efforts, in particular if the structural analysis is performed with the finite element method (FEM). A common methodology for fuzzy analysis with $\alpha$-discretization is vertex solution. Here, the extreme output responses to the FEMmodel for each $\alpha$-level are obtained by binary combinations of the fuzzy input variables; see, e.g., [Akpan et al. 2001]. Möller et al. [2000] employ a generic algorithm to perform the global optimization on every $\alpha$-level. Farkas et al. [2008] propose a parameter reduction scheme for the optimization problem. In order to accelerate optimization for the dynamic fuzzy FE analysis, De Munck et al. [2008] introduce a surrogate model, which replaces the real response of the analysis based on only a few computed values. Muhanna and Mullen [1999] employ the concept of $\alpha$-cuts within a fuzzy finite element method with applications for truss, beam, and plate problems in solid mechanics.

The above publications are typically formulated in terms of the underlying finite element discretized structure. To the author's knowledge, the variational formulation for fuzzy analysis of problems within the framework of continuum mechanics has not been considered so far. The present study intends to close this gap. Variational formulations have at least two intrinsic advantages:

- They enable a general framework for the analytical sensitivity analysis, which allows the effective solution of the related optimization problems with gradientbased methods; see, e.g., [Mahnken 2004]. 
- They give the basis for adaptive refinement of the underlying FE discretization [Rüter 2004; Johansson et al. 2007; Bangerth and Rannacher 2003; Widany and Mahnken 2012; Mahnken 2013].

Fuzzy analysis typically is performed for discrete values, e.g., the displacement at a certain finite element node. However, in some cases one might be interested in quantities within certain subregions of a structure. To this end, we introduce a so-called quantity of interest (or goal function) as an output quantity of the fuzzy analysis. Quantities of interest have been given much attention, e.g., in adaptive strategies over the last several years; see, e.g., [Rüter 2004; Johansson et al. 2007; Bangerth and Rannacher 2003; Widany and Mahnken 2012; Mahnken 2013].

This paper is structured as follows. Section 2 establishes the state problem and the target problem. Starting from basics of continuum mechanics, a weak formulation for the mechanical equilibrium is derived. Furthermore, we propose examples for a quantity of interest, which are used as output quantities for the fuzzy analysis. Section 3 incorporates the variational formulation into the optimization concepts of the extension principle and $\alpha$-level optimization. Section 4 provides the resulting optimality conditions in a variational setting. To this end, first- and second-order sensitivity analyses are performed by both the direct differentiation method as well as the adjoint state differentiation method. Moreover, we highlight the relation of least-squares problems to $\alpha$-level optimization. Section 5 briefly describes the finite element discretization followed by Section 6, which provides a brief overview on deterministic, gradient-based optimization methods. Representative examples in Section 7 demonstrate the versatility of the proposed variational formulation.

Notations. Symbolic tensor notation is employed mostly, if not stated otherwise. The scalar products of two vectors $\boldsymbol{a}$ and $\boldsymbol{b}$ as well as two second-order tensors $\boldsymbol{A}$ and $\boldsymbol{B}$ are, respectively,

$$
\boldsymbol{a} \cdot \boldsymbol{b}=a_{i} b_{i}, \quad \boldsymbol{A}: \boldsymbol{B}=A_{i j} B_{i j},
$$

using the Einstein summation rule for repeated indices $i, j=1,2,3$. The action of a second-order tensor $\boldsymbol{A}$ on a vector $\boldsymbol{b}$ is represented by $\boldsymbol{A} \cdot \boldsymbol{b}$. The tensor product $\boldsymbol{a} \otimes \boldsymbol{b}$ of two vectors $\boldsymbol{a}$ and $\boldsymbol{b}$ is a second-order tensor defined by the relation $(\boldsymbol{a} \otimes \boldsymbol{b}) \cdot \boldsymbol{b}=$ $(\boldsymbol{b} \cdot \boldsymbol{c}) \boldsymbol{a}$ for all vectors $\boldsymbol{c}$. 1 and $\square$ are the second- and fourth-order unit tensors, respectively, with the properties $\mathbf{1} \cdot \boldsymbol{c}=\boldsymbol{c}$ and $\square: \boldsymbol{C}=\boldsymbol{C}$ for all vectors $\boldsymbol{c}$ and all tensors $\boldsymbol{C}$. The nabla operator $\nabla$ with respect to initial coordinates $X$ is introduced, such that

$$
\nabla(\cdot)=\frac{\partial(\cdot)}{\partial X_{i}} \boldsymbol{e}_{i} \Rightarrow \nabla \boldsymbol{c} \equiv \boldsymbol{c} \otimes \nabla=c_{i, j} \boldsymbol{e}_{i} \otimes \boldsymbol{e}_{j} \quad \text { with } c_{i, j}=\frac{\partial c_{i}}{\partial X_{j}}
$$

where $\boldsymbol{e}_{i}, i=1,2,3$, is a Cartesian orthonormal basis and $X_{i}$ are coordinates of the reference placement $\boldsymbol{X}$. 


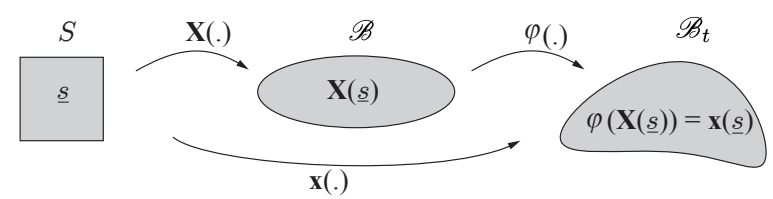

Figure 1. Mapping from design to configurations.

\section{State and target problem}

2.1. Strong and weak equilibrium problem. We consider a continuum body $\boldsymbol{B}$ occupying the reference (initial) configuration $\mathscr{B} \subset \mathscr{E}^{3}$ within the Euclidean space $\mathscr{E}^{3}$. Material particles are labeled by the placement vector $\boldsymbol{X} \in \mathscr{B}$. The surface of $\mathscr{B}$ is denoted as $\partial \mathscr{B}$, is assumed smooth, and has unit normal vector $N$. The time is denoted by $t \in \mathcal{T}$, where $\mathcal{T}=[0, T] \subset \mathbb{R}_{+}$is the time domain. Note that for time-independent problems the variable $t$ also provides a convenient history parameter in order to label the sequence of events and quasistatic conditions. In addition, we introduce $n_{s}$ design parameters $\left[s_{1}, \ldots, s_{n_{s}}\right]=\underline{s} \in \mathcal{S}$ within a design space $\mathcal{S}=\mathcal{S}_{1} \times \mathcal{S}_{2} \times \cdots \times \mathcal{S}_{n_{s}} \subset \mathbb{R}^{n_{s}}$. These may represent several influences on the structure, such as material, structural, or loading parameters, geometric properties or boundary conditions, etc. In this paper, the influence of uncertainty for the design parameters is taken into account by fuzzy analysis.

In order to relate design space $\mathcal{S}$ and time domain $\mathcal{T}$ as well as initial and current configurations $\mathscr{B}$ and $\mathscr{B}_{t}$, respectively, the following configurational mapping is introduced (see also [Barthold 1993] in the context of shape optimization):

$$
\boldsymbol{\varphi}(\cdot):=\left\{\begin{array}{c}
\mathscr{B} \times \mathcal{T} \times \mathcal{S} \rightarrow \mathscr{B}_{t} \subset \mathscr{E}^{3}, \\
\boldsymbol{X}(\underline{s}), t(\underline{s}), \underline{s} \mapsto \boldsymbol{x}(\underline{s})=\boldsymbol{\varphi}(\boldsymbol{X}(\underline{s}), t(\underline{s}), \underline{s}) .
\end{array}\right.
$$

The mappings $\boldsymbol{X}(\cdot): \mathcal{S} \rightarrow \mathscr{B} \subset \mathscr{E}^{3}$ and $t(\cdot): \mathcal{S} \rightarrow \mathcal{T} \subset \mathbb{R}_{+}$reflect dependencies of the initial geometry and the time, respectively, on design parameters. Here $\boldsymbol{x}(\cdot): \mathcal{S} \rightarrow \mathscr{B}_{t} \subset \mathscr{E}^{3}$ is a reduced placement operator, which relates the design space $\mathcal{S}$ and the actual configuration by means of the initial placement $\boldsymbol{X}$ and time $t$, such that $\boldsymbol{x} \in \mathscr{B}_{t}$ is the placement vector of a related particle at the current configuration $\mathscr{B}_{t} \subset \mathscr{E}^{3}$. The mappings $\boldsymbol{X}(\cdot), \boldsymbol{x}(\cdot)$, and $\varphi(\cdot)$ are visualized in Figure 1 , where for simplicity time dependence and explicit design dependence are not considered in the illustration.

Based on the configurational mapping in (3) we introduce three well known kinematical quantities of continuum mechanics

$$
\begin{aligned}
\boldsymbol{F} & =\nabla \boldsymbol{\varphi}(\boldsymbol{X}, t, \underline{s}), \\
J & =\operatorname{det} \boldsymbol{F}, \\
\boldsymbol{E} & =\frac{1}{2}\left(\boldsymbol{F}^{T} \cdot \boldsymbol{F}+\mathbf{1}\right),
\end{aligned}
$$


where $\boldsymbol{F}$ is the deformation gradient, which is known to be a mixed-variant, twofield tensor, $J$ is its determinant satisfying $J>0$, and $\boldsymbol{E}$ is the Green-Lagrange strain tensor.

Neglecting inertia terms, a strong formulation for the mechanical equilibrium problem is given as

$$
\begin{aligned}
& \text { Mechanical equilibrium } \quad \boldsymbol{\sigma} \cdot \nabla_{\boldsymbol{x}}+\overline{\boldsymbol{b}}(\underline{s})=\mathbf{0} \quad \text { in } \mathscr{B}_{t} \times \mathcal{S} \times \mathcal{T}, \\
& \text { Constitutive equations } \quad \sigma=\sigma(\varphi) \text { in } \mathscr{B}_{t} \times \mathcal{S} \times \mathcal{T}, \\
& \text { Boundary conditions } \quad \boldsymbol{u}=\overline{\boldsymbol{u}}(\underline{s}) \quad \text { on } \partial \mathscr{B}_{t u} \times \mathcal{S} \times \mathcal{T}, \\
& \boldsymbol{\sigma} \cdot \boldsymbol{n}=\overline{\boldsymbol{t}}(\underline{s}) \quad \text { on } \partial \mathscr{B}_{t \sigma} \times \mathcal{S} \times \mathcal{T} \text {. }
\end{aligned}
$$

Here, in addition to the previous notations we use $\sigma$ as the symmetric Cauchy stress tensor, $\nabla_{x}$ as the nabla operator with respect to the current placement vector $\boldsymbol{x}, \overline{\boldsymbol{b}}(\underline{s})$ as the body force vector per unit volume with respect to the current configuration, dependent on the (uncertain) design variables $\underline{s}$, and $\boldsymbol{u}=\boldsymbol{x}-\boldsymbol{X}$ as the displacement vector. The Dirichlet and Neumann boundary conditions (5c) and (5d), respectively, are formulated on the boundaries $\partial \mathscr{B}_{t u}$ and $\partial \mathscr{B}_{t \sigma}$, where the complementary conditions $\partial \mathscr{B}_{t u} \cup \partial \mathscr{B}_{t \sigma}=\partial \mathscr{B}_{t}$ and $\partial \mathscr{B}_{t u} \cap \partial \mathscr{B}_{t \sigma}=\varnothing$ hold, and where we defined traction forces $\overline{\boldsymbol{t}}(\underline{s})$ as well as prescribed displacements $\overline{\boldsymbol{u}}(\underline{s})$, both dependent on the (uncertain) design variables $\underline{s}$. In addition, $\boldsymbol{n}$ denotes a normal vector on the boundary $\partial \mathscr{B}_{t \sigma}$. A more general formulation for inelastic problems would require internal variables and their evolution equations, which however are left out here in order to alleviate the notation.

A weak formulation of the equilibrium problem is based on Hilbert spaces $\mathscr{U}$ for the displacement field $\boldsymbol{u} \in \mathscr{U}$ and $\mathscr{U}^{0} \subset \mathscr{U}$, which reflects zero Dirichlet boundary conditions for test functions $\delta \boldsymbol{u} \in \mathscr{U}^{0}$. Multiplying (5a) with test functions $\delta \boldsymbol{u} \in \mathscr{U}^{0}$, applying the divergence theorem, and incorporating the boundary conditions (5c)(5d), a weak formulation for the mechanical equilibrium is derived as

$$
\begin{aligned}
& \text { The state problem. For given } \underline{s} \in \mathcal{S} \text {, find } \boldsymbol{u}(\underline{s}) \in \mathscr{U} \text { such that } \\
& g(\underline{s}):=G(\underline{s}, \boldsymbol{u}(\underline{s}) ; \delta \boldsymbol{u}):=a(\underline{s}, \boldsymbol{u}(\underline{s}) ; \delta \boldsymbol{u})-\bar{l}(\delta \boldsymbol{u})=0 \text { for all } \delta \boldsymbol{u} \in \mathscr{U}^{0} .
\end{aligned}
$$

Here, the external and internal parts of the weak mechanical equilibrium equation are

$$
a(\boldsymbol{u}, \underline{s} ; \delta \boldsymbol{u})=\int_{\mathscr{B}_{t}} \boldsymbol{\sigma}: \boldsymbol{d}_{\delta} d v, \quad \bar{l}(\delta \boldsymbol{u})=\int_{\mathscr{B}_{t}} \overline{\boldsymbol{b}} \cdot \delta \boldsymbol{u} d v+\int_{\partial \mathscr{B}_{t \sigma}} \overline{\boldsymbol{t}} \cdot \delta \boldsymbol{u} d a,
$$

respectively, where $\boldsymbol{d}_{\delta}=\nabla_{x} \operatorname{sym}(\delta \boldsymbol{u})$ is a rate of deformation tensor, induced by the virtual displacement $\delta \boldsymbol{u} \in \mathscr{U}^{0}$; see Appendix B and Table B.1 for more details. Thus, $a(\cdot, \cdot ; \cdot)$ is a semilinear form on the Hilbert space $\mathcal{S} \times \mathscr{U} \times \mathscr{U}^{0}$, where linear and nonlinear dependent variables are separated by a semicolon. In the above, $\bar{l}(\cdot)$ 
is a linear functional with a dual pairing between the loading $\overline{\boldsymbol{b}}, \overline{\boldsymbol{t}} \in \mathscr{U}^{\prime}$ and $\boldsymbol{u} \in \mathscr{U}$, where $\mathscr{U}^{\prime}$ is the dual space of $\mathscr{U}$. The relation $G(\underline{s}, \boldsymbol{u}(\underline{s}) ; \delta \boldsymbol{u})=0$ is referred to as the state equation. We assume existence and uniqueness for the solution $\boldsymbol{u}(\underline{s})$ of the state problem (6), such that $g: \mathcal{S} \rightarrow \mathbb{R}$ defines a reduced solution operator.

For iterative solution of (6), with "frozen" design variables $\underline{s}$, we employ a Newton method at the current state $\tilde{\boldsymbol{u}}$ :

$$
\begin{aligned}
G_{\boldsymbol{u}}^{\prime}(\underline{s}, \tilde{\boldsymbol{u}}(\underline{s}) ; \delta \boldsymbol{u}, \Delta \boldsymbol{u}) & =-G(\underline{s}, \tilde{\boldsymbol{u}}(\underline{s}) ; \delta \boldsymbol{u}) \quad \text { for all } \delta \boldsymbol{u} \in \mathscr{U}^{0} \\
\Longrightarrow \boldsymbol{u} & =\tilde{\boldsymbol{u}}+\Delta \boldsymbol{u} .
\end{aligned}
$$

The generally unsymmetric tangent bilinear form $G_{\boldsymbol{u}}^{\prime}(\cdot, \cdot ; \cdot, \cdot)$ denotes the partial derivative with respect to $\boldsymbol{u}$. It is defined as a Gâteaux derivative at the point $\tilde{\boldsymbol{u}}$ in the direction of the virtual variation $\Delta \boldsymbol{u}$, i.e.,

$$
G_{\boldsymbol{u}}^{\prime}(\underline{s}, \tilde{\boldsymbol{u}} ; \delta \boldsymbol{u}, \Delta \boldsymbol{u}):=\lim _{\varepsilon \rightarrow 0} \varepsilon^{-1}(G(\underline{s}, \tilde{\boldsymbol{u}}+\varepsilon \Delta \boldsymbol{u} ; \delta \boldsymbol{u})-G(\underline{s}, \tilde{\boldsymbol{u}} ; \delta \boldsymbol{u})),
$$

assuming that the limit exists. The solution $\Delta \boldsymbol{u}$ of (8a) renders the new iterate $\boldsymbol{u}$ in (8b). More detailed formulations of the state equation $G_{\boldsymbol{u}}(\cdot, \cdot ; \cdot)$ and the tangent bilinear form $G_{\boldsymbol{u}}^{\prime}(\cdot, \cdot ; \cdot, \cdot)$ are provided in Appendix B.

2.2. Quantity of interest and target problem. In the subsequent exposition we do not merely want to characterize the fuzzy properties of the displacement field $\boldsymbol{u}(\underline{s})$ satisfying the state problem (6). Instead we are more interested in the fuzzy analysis of a physical event or a feature of the structure that depends upon $\boldsymbol{u}$. Such quantities of interest are characterized by functionals $Q(\boldsymbol{u})$ of the solutions $\boldsymbol{u}$ to (6). Quantities of interest have been given much attention in adaptive strategies over the last several years; see, e.g., [Rüter 2004; Johansson et al. 2007; Bangerth and Rannacher 2003; Widany and Mahnken 2012; Mahnken 2013] and, in the context of multiscale modeling, [Oden et al. 2006]. Possible realizations of the quantity of interest may be

(1) displacement at a certain point of the body $P \in \boldsymbol{B}$ with coordinates $\boldsymbol{X}_{P} \in \mathscr{B}$ at the end of loading with time $t=T$

$$
Q_{X}(\boldsymbol{u})=\left\|\boldsymbol{u}\left(\boldsymbol{X}_{P}, T\right)\right\|_{\mathcal{D}},
$$

(2) displacements at certain regions $\mathscr{B}_{m} \subset \mathscr{B}$ and certain time intervals $\mathcal{T}_{n} \subset \mathcal{T}$

$$
Q_{u}(\boldsymbol{u})=\int_{\mathcal{T}_{n}} \int_{\mathscr{B}_{m}}\|\boldsymbol{u}\|_{\mathcal{D}} d V d t
$$

(3) strains, e.g., of Green-Lagrange type according to (4), at certain regions $\mathscr{B}_{m} \subset$ $\mathscr{B}$ and certain time intervals $\mathcal{T}_{n} \subset \mathcal{T}$

$$
Q_{E}(\boldsymbol{u})=\int_{\mathcal{T}_{n}} \int_{\mathscr{B}_{m}}\|\boldsymbol{E}(\boldsymbol{u})\|_{\mathcal{D}} d V d t
$$


(4) stresses at certain regions $\mathscr{B}_{m} \subset \mathscr{B}$ and certain time intervals $\mathcal{T}_{n} \subset \mathcal{T}$

$$
Q_{P}(\boldsymbol{u})=\int_{\mathcal{T}_{n}} \int_{\mathscr{B}_{m}}\|\boldsymbol{P}(\boldsymbol{u})\|_{\mathcal{D}} d V d t
$$

and where $\boldsymbol{P}=J \boldsymbol{\sigma} \cdot \boldsymbol{F}^{-T}$ is the first Piola-Kirchhoff stress tensor,

(5) stresses at the boundary of prescribed displacements $\partial \mathscr{B}_{u} \subset \partial \mathscr{B}$ and time intervals $\mathcal{T}_{n} \subset \mathcal{T}$

$$
Q_{F}(\boldsymbol{u})=\int_{\mathcal{T}_{n}} \int_{\partial \mathscr{B}_{u}}\left\|\boldsymbol{P}(\boldsymbol{u})^{T} \cdot \boldsymbol{N}\right\|_{\mathcal{D}} d A d t
$$

such that the goal function has the interpretation of (a sum of) reaction forces,

(6) functional dependencies of design variables

$$
Q_{s}=Q(\underline{s})
$$

(7) or a combination of the above quantities with adequate weighting.

The standard choice of norm $\|\cdot\|_{\mathcal{D}}$ in the above definitions is a weighted $L^{2}$-norm.

Moreover, a comparison of the state variable $\boldsymbol{u}(\underline{s}) \in \mathscr{U}$ with experimental data $\boldsymbol{u}^{*} \in \mathscr{U}^{*}$ could be integrated in the quantity of interest, where $\mathscr{U}^{*}$ is the space of true data, that is, without measurement errors. To this end, we define the mappings

$$
\begin{aligned}
& \underline{\bar{d}}=\underline{\bar{D}}\left(\boldsymbol{u}^{*}\right) \quad \in \mathcal{D} \subset \mathbb{R}^{n_{d}}, \\
& \underline{d}(\underline{s}):=\underline{D}(\boldsymbol{u}(\underline{s})) \in \mathcal{D} \subset \mathbb{R}^{n_{d}} \text {. }
\end{aligned}
$$

Equation (15a) introduces an observation operator $\underline{\bar{D}}: \mathscr{U}^{*} \rightarrow \mathcal{D}$ mapping the trajectory of true data $\boldsymbol{u}^{*}$ to points $\underline{\bar{d}}=\underline{\bar{D}}\left(\boldsymbol{u}^{*}\right)$ in the data space $\mathcal{D} \in \mathbb{R}^{n_{d}}$ [Banks and Kunisch 1989]. Equation (15b) introduces a second observation operator $\underline{D}: \mathscr{U} \rightarrow \mathcal{D}$, which maps the state variable $\boldsymbol{u}(\underline{s}) \in \mathscr{U}$ to points $\underline{D}(\boldsymbol{u}(\underline{s}))$ in the data space $\mathcal{D} \in \mathbb{R}^{n_{d}}$. Moreover, in order to point out the "design driven" data as a result of the state problem (6), (15b) introduces the reduced observation operator $\underline{d}: \mathcal{S} \rightarrow \mathcal{D}$ to transform design variables $\underline{s}$ to the observation space [Vexler 2004]. In index notation (15b) can be written as $d_{k}(\underline{s}):=D_{k}(\boldsymbol{u}(\underline{s})), k=1, \ldots, n_{d}$. Hereafter, we assume $n_{d} \geq n_{s}$ for the number of experimental data $n_{d}$ and for the number of design variables $n_{s}$ (in this case material parameters), and we assume both operators $\underline{D}$ and $\underline{D}$ to be linear. With these notations a model error with respect to the data space $\mathcal{D} \subset \mathbb{R}^{n_{d}}$ becomes

$$
\underline{e}_{\mathrm{Mod}}=\underline{D}(\boldsymbol{u}(\underline{s}))-\underline{\bar{D}}\left(\boldsymbol{u}^{*}\right)=\underline{d}(\underline{s})-\underline{\bar{d}} \in \mathcal{D} \subset \mathbb{R}^{n_{d}}
$$


as the difference of simulated and experimental data. Using a weighted $L^{2}$-norm a quantity of interest may become

$$
\begin{array}{r}
Q_{\mathrm{LS}}(\boldsymbol{u}(\underline{s}))=\frac{1}{2} \int_{\mathcal{T}_{n}} \int_{\mathscr{B}_{m}}\left\|\underline{D}(\boldsymbol{u}(\underline{s}))-\underline{\bar{D}}\left(\boldsymbol{u}^{*}\right)\right\|_{\mathcal{D}}^{2} d V d t=\frac{1}{2}\|\underline{W}(\underline{d}(\underline{s}))-\underline{\bar{d}}\|_{2}^{2} \\
=: q_{\mathrm{LS}}(\underline{s}) .
\end{array}
$$

In the third part of (17) we have introduced a weighting matrix $\underline{W} \in \mathbb{R}^{n_{d} \times n_{d}}$, which accounts, e.g., for different physical dimensions or reasonable scaling for the quantities of interest on the region $\mathscr{B}_{m} \subset \mathscr{B}$ and the time interval $\mathcal{T}_{n} \subset \mathcal{T}$. Analogously to the reduced solution operator $g: \mathcal{S} \rightarrow \mathbb{R}$ in (6), $q_{\mathrm{LS}}: \mathcal{S} \rightarrow \mathbb{R}$ in (17) represents a reduced quantity of interest operator. Of course, the quantity of interest in (17) is nothing else but the least-squares functional in identification problems. Here, the interest is to minimize the distance between simulated data and experimental data in a least-squares sense; see, e.g., [Mahnken 2004]. Upon neglecting measurement errors the functional in (17) also has the interpretation of a mean for the model error in (16).

With a specific choice for the quantity of interest $Q$ at hand, for example according to (10)-(14) or (17), we formulate:

The target problem. For given $\underline{s} \in \mathcal{S}$, find $z:=q(\underline{s}):=Q(\underline{s}, \boldsymbol{u}(\underline{s}))$.

Note that due to the dependency $\boldsymbol{u}(\underline{s})$, solution of the target problem (18) involves solution of the state problem (6). In the following exposition, we assume a continuous mapping for the reduced quantity of interest operator $q: \mathcal{S} \rightarrow \mathbb{R}$ between the design variables $\underline{s}$ and the quantity of interest $q$ and a unique solution $\boldsymbol{u}(\underline{s})$.

\section{Fuzzy analysis}

3.1. Fuzzy set and fuzzy number. So far, the design space $\mathcal{S} \subset \mathbb{R}^{n_{s}}$ in the previous section has been assumed a precise set or a fundamental set [Möller and Beer 2004] of ordered pairs

$$
\begin{aligned}
\mathcal{S} & =\left\{\left(\underline{s}, \underline{\xi}_{\mathcal{S}}(\underline{s})\right) \mid \underline{s} \in \mathbb{R}^{n_{s}}, \underline{\xi}_{S}(\underline{s})=\{0,1\}^{n_{s}}\right\} \\
& =\mathcal{S}_{1} \times \mathcal{S}_{2} \times \cdots \times \mathcal{S}_{n_{s}} \subset \mathbb{R}^{2 n_{s}}, \\
\text { where } \quad \mathcal{S}_{i} & =\left\{\left(s_{i}, \xi_{\mathcal{S} i}\left(s_{i}\right)\right) \mid s_{i} \in \mathbb{R}, \xi_{\mathcal{S i}}\left(s_{i}\right) \in\{0,1\}\right\} \subset \mathbb{R}^{2}, \\
\xi_{\mathcal{S} i} & : \mathbb{R} \rightarrow\{0,1\}, \quad s_{i} \mapsto \begin{cases}1 & \text { if } s_{i} \in \mathcal{S}_{i}, \\
0 & \text { if } s_{i} \notin \mathcal{S}_{i} .\end{cases}
\end{aligned}
$$

According to (19b) the Cartesian product $\mathcal{S}$ comprises all combinations of the design variables $s_{1}, \ldots, s_{n_{s}}$ of the precise sets $\mathcal{S}_{i}$ in (19c). The corresponding indicator functions $\xi_{\mathcal{S} i}$ (19d) specify crisp interval boundaries for each design variable $s_{i}, i=1, \ldots, n_{s}$, in a precise manner; see, e.g., [Viertl 1996]. However, due to 

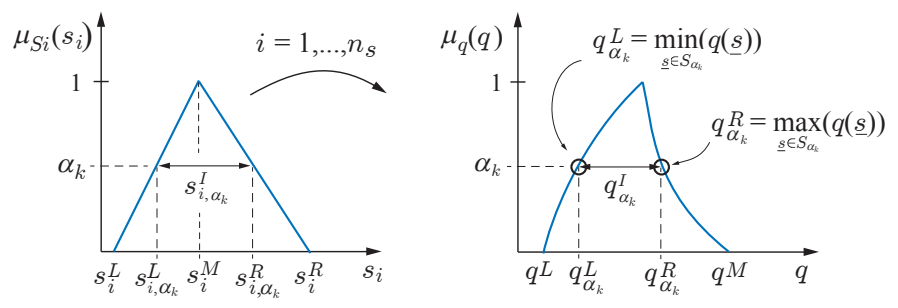

Figure 2. Schematic graph of fuzzy analysis with $\alpha$-level optimization. Left: input membership function, fuzzy triangular number $\hat{A}_{i}=\left\langle s_{i}^{L}, s_{i}^{M}, s_{i}^{R}\right\rangle$, and $\alpha$-levels. Right: output membership function.

imprecision of measurements this is not realistic. Therefore, in fuzzy analysis, the precise set $\mathcal{S}$ is replaced by a nonprecise set, or fuzzy set [Möller and Beer 2004] of ordered pairs

$$
\begin{aligned}
\hat{\mathcal{S}} & =\left\{\left(\underline{s}, \mu_{S}(\underline{s})\right) \mid \underline{s} \in \mathbb{R}^{n_{s}}, \mu_{S}(\underline{s})=\min _{i=1, \ldots, n_{s}}\left\{\mu_{\mathcal{S} i}\left(\underline{s}_{i}\right)\right\}\right\} \\
& =\hat{\mathcal{S}}_{1} \times \hat{\mathcal{S}}_{2} \times \cdots \times \hat{\mathcal{S}}_{n_{s}} \subset \mathbb{R}^{2 n_{s}}, \\
\hat{\mathcal{S}}_{i} & =\left\{\left(s_{i}, \mu_{\mathcal{S} i}\left(s_{i}\right)\right) \mid s_{i} \in \mathbb{R}, \mu_{\mathcal{S} i}\left(s_{i}\right) \in[0,1]\right\} \subset \mathbb{R}^{2}, \\
\mu_{\mathcal{S} i} & : \mathbb{R} \rightarrow[0,1], \quad s_{i} \mapsto 0 \leq \mu_{\mathcal{S} i}\left(\underline{s}_{i}\right) \leq 1 .
\end{aligned}
$$

According to (20a) and (20b) the Cartesian product $\hat{\mathcal{S}}$ comprises all combinations of the design variables $s_{1}, \ldots, s_{n_{s}}$. Equation (20c) is the one-dimensional fuzzy set where in (20d), for each set $\hat{\mathcal{S}}_{i}$ the indicator function $\xi_{\mathcal{S} i}\left(s_{i}\right)$ in $(19 \mathrm{~d})$ is replaced by a membership function $\mu_{\mathcal{S} i}\left(s_{i}\right)$, which represents a gradual weighting of each design space $\mathcal{S}_{i}$. Subsequently, only normalized membership functions with the property $\sup _{s_{i} \in \mathcal{S}_{i}}\left[\mu_{\mathcal{S} i}\left(s_{i}\right)\right]=1$ are considered. The closer $\mu_{\mathcal{S} i}\left(s_{i}\right)$ is to 1 , the more the element $s_{i}$ belongs to the set $\hat{\mathcal{S}}_{i}$; the closer $\mu_{\mathcal{S} i}\left(s_{i}\right)$ is to 0 , the less the element $s_{i}$ belongs to the set $\hat{\mathcal{S}}_{i}$. A convex and normalized fuzzy set is referred to as a fuzzy number. Figure 2, left, illustrates a triangular fuzzy number

$$
\hat{A}_{i}=\left\langle s_{i}^{L}, s_{i}^{M}, s_{i}^{R}\right\rangle, \quad \text { where } s_{i}^{L}<s_{i}^{M}<s_{i}^{R},
$$

with in-between value $s_{i}^{M}$ and lower and upper bounds $s_{i}^{L}, s_{i}^{R}$.

By definition of the reduced quantity of interest operator $q: \mathcal{S} \rightarrow \mathbb{R}$ in (17), the quantities of interest $Q$ in (10)-(14) and (17) for displacements, stresses, strains, etc., become functions of the design variables $s_{i}$ and consequently can be treated as fuzzy functions. The intrinsic goal of fuzzy analysis is to propagate the uncertainties of the fuzzy input variables, in our case the design variables $s_{i}, i=1, \ldots, n_{s}$, to a fuzzy output variable, in our case the quantity of interest $q(\underline{s})$ in the target problem (18). 
3.2. The extension principle. In order to perform mathematical operations with fuzzy numbers Zadeh developed the extension principle [Dubois and Prade 1980; Zadeh 1965; Möller and Beer 2004]. It specifies the mapping of the fuzzy input set $\hat{\mathcal{S}}$ into a new fundamental set $\mathcal{Z}$ with the aid of the mapping $z=q(\underline{s})$ defined in the target problem (18). On the fundamental set $\mathcal{Z}$ the fuzzy set $\tilde{\mathcal{B}}$ together with its membership function $\mu_{B}(z)$ is gained. The mapping $q(\underline{s})$ leads to the fuzzy set $\tilde{\mathcal{B}}$ on $\mathcal{Z}$ :

$$
\tilde{\mathcal{B}}=\left\{\left(z, \mu_{B}(z)\right) \mid z=q(\underline{s}), z \in \mathcal{Z} ; \underline{s} \in \mathcal{S}\right\},
$$

where

$$
\mu_{B}(z)= \begin{cases}\sup _{z=q(\underline{s})} \min \left(\mu_{i}\left(s_{1}\right), \ldots, \mu_{n_{s}}\left(s_{n_{s}}\right)\right) & \text { if there exists } z=q(\underline{s}), \\ 0 & \text { otherwise. }\end{cases}
$$

Here the membership values $\mu_{B}(z)$ are computed according to the sup-min problem (22b) in order to minimize and maximize the quantity of interest $q(\underline{s})$ defined in (18). Following Akpan et al. [2001] problem (22) can be interpreted as follows. For a crisp value of the output $z$, there exist zero, one, or more combinations of crisp values of input variables $\underline{s}$ such that $z=q(\underline{s})$. The possibility of each combination is given by the smallest possibility value for all fuzzy input variables. The possibility that $z=q(\underline{s})$ is given by the maximum possibility value for all these combinations.

As pointed out by Wasfy and Noor [1998] the direct solution of (22b) is intractable except for very simple fuzzy finite element problems involving at most one or two fuzzy variables. For a general function $q$, the number of operations needed to solve the state problem (6) is of the order $\infty^{n_{s}}$ [Wasfy and Noor 1998].

3.3. $\alpha$-level optimization. Due to its mathematical complexity and the significant numerical effort the extension principle, (22) can be approximated by the so-called $\alpha$-level optimization method. Here, as schematically illustrated in Figure 2, left, each membership input function $\mu_{i}$ is discretized into $n_{\alpha} \alpha$-cuts, where $0 \leq \alpha_{k} \leq 1$ denotes the membership level [Möller and Beer 2004]. For this purpose we define the crisp set

$$
\mathcal{S}_{i, \alpha_{k}}=\left\{s_{i} \mid \mu_{\mathcal{S} i, \alpha_{k}}\left(s_{i}\right) \geq \alpha_{k}, s_{i} \in \mathcal{S}_{i}\right\}=\left[s_{i, \alpha_{k}}^{L}, s_{i, \alpha_{k}}^{R}\right]:=s_{i, \alpha_{k}}^{I}, \quad i=1, \ldots, n_{s},
$$

of the fuzzy variable $s_{i}$ at level $\alpha_{k}$. It has crisp lower and upper bounds $s_{i, \alpha_{k}}^{L}$ and $s_{i, \alpha_{k}}^{R}$, respectively, and therefore defines a crisp interval as illustrated in Figure 2, left. Note also that the $\alpha$-cut $\mathcal{S}_{i, 0}=\left[s_{i}^{L}, s_{i}^{R}\right]$ is the support of the fuzzy set $\hat{\mathcal{S}}_{i}$. The intervals in (23) give rise to the definition of an $n_{s}$-dimensional constrained design space

$$
\mathcal{S}_{\alpha_{k}}=\left[\mathcal{S}_{1, \alpha_{k}} \times \cdots \times \mathcal{S}_{i, \alpha_{k}} \times \cdots \times \mathcal{S}_{n_{s}, \alpha_{k}}\right] \subset \mathcal{S} \subset \mathbb{R}^{n_{s}} .
$$


Then, the fuzzy response for each selected $\alpha_{k}$-level is obtained from the minimum and maximum values of the quantity of interest $q$ in (18) as

$$
\begin{aligned}
& \text { P1: } \alpha \text {-level optimization problems. } \\
& \text { Find } q_{\alpha_{k}}^{L}=\min _{\underline{s} \in \mathcal{S}_{\alpha_{k}}} q(\underline{s})=\min _{\underline{s} \in \mathcal{S}_{\alpha_{k}}} Q(\underline{s}, \boldsymbol{u}(\underline{s})) . \\
& \text { Find } q_{\alpha_{k}}^{R}=\max _{\underline{s} \in \mathcal{S}_{\alpha_{k}}} q(\underline{s})=\max _{\underline{s} \in \mathcal{S}_{\alpha_{k}}} Q(\underline{s}, \boldsymbol{u}(\underline{s})) .
\end{aligned}
$$

In this way, fuzzy analysis reduces to an interval-based analysis with crisp bounds. As visualized in Figure 2, right, the two extrema $q_{\alpha_{k}}^{L}$ and $q_{\alpha_{k}}^{R}$ render two points of the membership function $\mu_{q}(q)$ for the membership level $\alpha_{k}$. As, by assumption in Section 2.1, there is a continuous mapping $\boldsymbol{u}=\boldsymbol{u}(\underline{s})$ between the design variables $\underline{s}$ and the quantity of interest $q$ with a unique solution of the state problem (6), the interval

$$
q_{\alpha_{k}}^{I}=\left[q_{\alpha_{k}}^{L}, q_{\alpha_{k}}^{R}\right]
$$

is fully described by the lower and upper bounds $q_{\alpha_{k}}^{L}$ and $q_{\alpha_{k}}^{R}$. In addition we remark that due to the definition for $\mathcal{S}_{\alpha_{k}} \subset \mathbb{R}^{n_{s}}$ in (24) both optimization problems in (25) involve only simple constraints. Problems of this kind are treated extensively in [Bertsekas 1982].

\section{Optimality conditions for the $\alpha$-level optimization problems}

4.1. Preliminaries. The optimization problems (25a) and (25b) differ only in the specification of a minimum and a maximum. Therefore, without loss of generality following this section we will mainly resort to the minimum problem (25a), if not stated otherwise. At the same time, for notational brevity the upper index $L$ and the lower index $\alpha_{k}$ are left out. We also note that the present article is focused on the case of simple constraints in (23). This means that no interaction of the fuzzy variables as in the quantity of interest in (14) is considered, which will be a further challenge in future work on fuzzy analysis; see, e.g., [Möller and Beer 2004].

First-order necessary and second-order sufficient optimality conditions for a stable stationary point $\underline{s} \in \mathcal{S}_{\alpha_{k}}$ of the minimization problem (25a) are

$$
\begin{aligned}
q^{\prime}(\underline{s} ; \delta \underline{s}) & \geq 0, \\
q^{\prime \prime}(\underline{s} ; \delta \underline{s}, \delta \underline{s}) & \geq \gamma \| \delta \underline{s}_{\mathcal{S}_{\alpha_{k}}}^{2} \quad \text { for all } \delta \underline{s} \in \overline{\mathcal{S}}_{\alpha_{k}},
\end{aligned}
$$

where

$$
\overline{\mathcal{S}}_{\alpha_{k}}(\underline{s})=\left\{\delta \underline{s} \in \mathcal{S} \mid \text { there exists } \bar{\beta}>0 \text { such that } \underline{s}+\beta \delta \underline{s} \in \mathcal{S}_{\alpha_{k}}, 0 \leq \beta<\bar{\beta}\right\}
$$


is the space of admissible directions. Furthermore, in (27) we have $\gamma \in \mathbb{R}_{+}$; see, e.g., [Nocedal and Wright 1999]. For computing $q^{\prime}(\underline{s} ; \delta \underline{s})$ and $q^{\prime \prime}(\underline{s} ; \delta \underline{s})$ two conceptions are distinguished: the (more conventional) direct differentiation method and the (more formal) adjoint state method.

4.2. The direct differentiation method. Application of the chain rule to the reduced functionals in (6) and (25a) gives us

$$
\begin{array}{ll}
g^{\prime}(\underline{s} ; \delta \underline{s})=G_{\underline{s}}^{\prime}(\underline{s}, \boldsymbol{u}(\underline{s}) ; \delta \boldsymbol{u}, \delta \underline{s})+G_{\boldsymbol{u}}^{\prime}(\underline{s}, \boldsymbol{u}(\underline{s}) ; \delta \boldsymbol{u}, \bar{\delta} \boldsymbol{u})=0 & \text { for all } \delta \boldsymbol{u} \in \mathscr{U}^{0} \\
& \text { and } \delta \underline{s} \in \mathcal{S}_{\alpha_{k}} \\
q^{\prime}(\underline{s} ; \delta \underline{s})=Q_{\underline{s}}^{\prime}(\underline{s}, \boldsymbol{u}(\underline{s}) ; \delta \underline{s})+Q_{\boldsymbol{u}}^{\prime}(\underline{s}, \boldsymbol{u}(\underline{s}) ; \bar{\delta} \boldsymbol{u}) \quad \geq 0 & \text { for all } \delta \underline{s} \in \mathcal{S}_{\alpha_{k}}
\end{array}
$$

where $G_{s}^{\prime}(\cdot, \cdot ; \cdot), G_{\boldsymbol{u}}^{\prime}(\cdot, \cdot ; \cdot, \cdot)$, etc., denote partial derivatives with respect to $\underline{\underline{s}}$ and $\boldsymbol{u}$, defined as Gâteaux derivatives. The tangent problem in (29a) is zero since $\boldsymbol{u}(\underline{s})$ solves the state problem (6) for each $\underline{s} \in \mathcal{S}$, where $\bar{\delta} \boldsymbol{u}:=\boldsymbol{u}_{s}^{\prime}(\underline{s} ; \delta \underline{s})$ is the directional sensitivity along $\delta s$ [Johansson et al. 2007]. The inequality (29b) follows from the first-order optimality condition (27a). Inserting the solution $\bar{\delta} \boldsymbol{u}$ of the tangent problem (29a) into (29b) may be used to evaluate $q^{\prime}(\underline{s} ; \delta \underline{s})$. With this result for $q^{\prime}$ we introduce the gradient of the quantity of interest

$$
\nabla q(\underline{s}) \in \mathcal{S}, \quad \text { where }(\nabla q(\underline{s}))_{i}=q^{\prime}\left(\underline{s} ; \underline{e}_{i}\right), i=1, \ldots, n_{s},
$$

and where $\left(\underline{e}_{i}\right)_{j}=\delta_{i j}$ with the standard Kronecker delta. Then, for the case of simple constraints in (24), the necessary conditions (27a) and (29b) simplify as [Bertsekas 1982]

$$
\{\nabla q(\underline{s})\}_{i}=\frac{\partial q}{\partial s_{i}} \begin{cases}=0 & \text { if } s_{i, \alpha_{k}}^{L}<s_{i}<s_{i, \alpha_{k}}^{R} \\ \geq 0 & \text { if } s_{i}=s_{i, \alpha_{k}}^{L}, \\ \leq 0 & \text { if } s_{i}=s_{i, \alpha_{k}}^{R}\end{cases}
$$

A detailed study of the second-order optimality condition (27b) by means of the direct differentiation method is performed in Appendix A.1.

As an example, we consider the quantity of interest of least-squares type in (17). Exploiting $Q_{\underline{s}}^{\prime}(\underline{s}, \boldsymbol{u}(\underline{s}) ; \delta \underline{s})=0$ in $(29 \mathrm{~b})$, the gradient is derived from (29b) as

$$
\begin{aligned}
\nabla q_{\mathrm{LS}}(\underline{s}) & =(\underline{d}(\underline{s})-\underline{\bar{d}})^{T} \underline{W}^{T} \underline{W} \underline{J}, \\
\text { where } \underline{J} & =\nabla \underline{d}(\underline{s})=\underline{D}_{\boldsymbol{u}}^{\prime}(\boldsymbol{u}) \cdot \nabla \boldsymbol{u}(\underline{s}) .
\end{aligned}
$$

In (32b), the Jacobian matrix $\underline{J}$ is obtained by the chain rule applied to the reduced observation operator in (15) with relations $(\nabla \boldsymbol{u}(\underline{s}))_{i}=\boldsymbol{u}_{s}^{\prime}\left(\underline{s} ; \underline{e}_{i}\right)$. Moreover, exploiting $Q_{\underline{s} \underline{s}}^{\prime \prime}\left(\underline{s}, \boldsymbol{u}(\underline{s}) ; \delta \underline{s}_{1}, \delta \underline{s}_{2}\right)=Q_{\underline{s} \boldsymbol{u}}^{\prime \prime}\left(\underline{s}, \boldsymbol{u}(\underline{s}) ; \delta \underline{s}_{1}, \bar{\delta} \boldsymbol{u}_{2}\right)=\bar{Q}_{\underline{u} \underline{\underline{s}}}^{\prime \prime}\left(\underline{s}, \boldsymbol{u}(\underline{s}) ; \bar{\delta} \boldsymbol{u}_{1}, \bar{\delta} \boldsymbol{u}_{2}\right)=0$ in (A-1b), the Hessian of the reduced cost functional $q_{\mathrm{LS}}$ in (25a) is derived from 
$(\mathrm{A}-1 \mathrm{~b})$ as

$$
\begin{aligned}
& \nabla^{2} q_{\mathrm{LS}}(\underline{s})=\underline{J}^{T} \underline{W}^{T} \underline{W} \underline{J}+\underline{M} \\
& \text { where } \underline{M}=\sum_{i, j, k=1}^{n_{d}} W_{i j}\left(d_{j}(\underline{s})-\bar{d}_{j}\right) W_{i k}\left(D_{k}\right)_{\boldsymbol{u}}^{\prime}(\boldsymbol{u}) \cdot \nabla^{2} \boldsymbol{u}(\underline{s})
\end{aligned}
$$

with relations $\left(\nabla^{2} \boldsymbol{u}(\underline{s})\right)_{i j}=\boldsymbol{u}_{s S}^{\prime \prime}\left(\underline{s} ; \underline{e}_{i}, \underline{e}_{j}\right)$.

4.3. The adjoint state differentiation method. Let us consider the optimization problems

$$
\begin{aligned}
& \text { P2: } \alpha \text {-level optimization problems. } \\
& \text { (1) Find } Q_{\alpha_{k}}^{L}=\min _{\underline{s} \in \mathcal{S}_{\alpha_{k}}, \boldsymbol{u} \in \mathscr{U}} Q(\underline{s}, \boldsymbol{u}) \text {. (2) Find } Q_{\alpha_{k}}^{R}=\max _{\underline{\underline{S}} \in \mathcal{S}_{\alpha_{k}}, \boldsymbol{u} \in \mathscr{U}} Q(\underline{s}, \boldsymbol{u}) . \\
& G(\underline{s}, \boldsymbol{u} ; \delta \boldsymbol{u})=0 \text { for all } \delta \boldsymbol{u} \in \mathscr{U}^{0} . \\
& g_{j}=s_{j, \alpha_{k}}^{L}-s_{j} \leq 0, j=1, \ldots, n_{s} . \\
& g_{n_{s}+j}=s_{j}-s_{j, \alpha_{k}}^{R} \leq 0, j=1, \ldots, n_{s} .
\end{aligned}
$$

in order to minimize and maximize the quantity of interest $Q$. Equation (34b) constitutes the state problem (6) as an equality condition, and the inequalities (34c)-(34d) specify constraint functions associated to the $n_{s}$-dimensional space $\mathcal{S}_{\alpha_{k}}$ in (24). Contrary to problem P1 in (25), the design variables $\underline{s}$ and the displacement field $\boldsymbol{u}$ are independent variables. Consequently, there is no functional dependence $\boldsymbol{u}(\underline{s})$, and so far no use is made of the reduced quantity of interest operator $q: \mathcal{S} \rightarrow \mathbb{R}$ in the target problem (18) and the reduced solution operator $g: \mathcal{S} \rightarrow \mathbb{R}$ in the state problem (6). As in Section 4, without loss of generality, from now on we will resort only to the minimum problem with quantity of interest in (34a)(1). If there is no risk of confusion, the lower index $\alpha_{k}$ is left out for notational brevity.

A Lagrange function $\mathcal{L}_{\alpha_{k}}\left(\underline{s}_{\alpha_{k}}, \boldsymbol{u}_{\alpha_{k}}, \boldsymbol{\Lambda}_{\alpha_{k}}, \underline{\lambda}_{\alpha_{k}}\right) \equiv \mathcal{L}(\underline{s}, \boldsymbol{u}, \boldsymbol{\Lambda}, \underline{\lambda})$ related to problem P2(1) in (34) is formulated where the test function $\Lambda \in \mathscr{U}^{0}$ acts as a Lagrange multiplier for the equality constraint (34b), and the scalars $\underline{\lambda} \in \mathcal{S}^{2}$ act as Lagrange multipliers for the inequality constraints (34c)-(34d):

$$
\mathcal{L}(\underline{s}, \boldsymbol{u}, \boldsymbol{\Lambda}, \underline{\lambda}):=Q(\underline{s}, \boldsymbol{u})+G(\underline{s}, \boldsymbol{u} ; \boldsymbol{\Lambda})+\sum_{j=1}^{2 n_{s}} \lambda_{j} g_{j}(\underline{s}) \rightarrow \operatorname{stat}_{z \in \mathscr{Z}},
$$

where $(\underline{s}, \boldsymbol{u}, \boldsymbol{\Lambda}, \underline{\lambda})=: z \in \mathscr{Z}=: \mathcal{S} \times \mathscr{U} \times \mathscr{U}^{0} \times \mathcal{S}^{2}$. The quadruple $(\underline{s}, \boldsymbol{u}, \boldsymbol{\Lambda}, \underline{\lambda})$ is a local solution of the optimization minimum problem (34) provided the following 
Karush-Kuhn-Tucker conditions hold [Luenberger 1984]:

$$
\begin{array}{rlrl}
\mathcal{L}_{\underline{s}}^{\prime}(z ; \delta \underline{s}) & =Q_{\underline{s}}^{\prime}(\underline{s}, \boldsymbol{u} ; \delta \underline{s})+G_{\underline{s}}^{\prime}(\underline{s}, \boldsymbol{u} ; \boldsymbol{\Lambda}, \delta \underline{s})+\sum_{j=1}^{2 n_{s}} \lambda_{j} \nabla_{\underline{s}} g_{j}(\underline{s}) \cdot \delta \underline{s}=0 \\
& \text { for all } \delta \underline{s} \in \mathcal{S}, \\
\mathcal{L}_{\boldsymbol{u}}^{\prime}(z ; \delta \boldsymbol{u}) & =Q_{\underline{u}}^{\prime}(\underline{s}, \boldsymbol{u} ; \delta \boldsymbol{u})+G_{\boldsymbol{u}}^{\prime}(\underline{s}, \boldsymbol{u} ; \boldsymbol{\Lambda}, \delta \boldsymbol{u})=0 & \text { for all } \delta \boldsymbol{u} \in \mathscr{U}^{0}, \\
\mathcal{L}_{\Lambda}^{\prime}(z ; \delta \boldsymbol{\Lambda}) & =G_{\Lambda}^{\prime}(\underline{s}, \boldsymbol{u} ; \boldsymbol{\Lambda}, \delta \boldsymbol{\Lambda})=a(\underline{s}, \boldsymbol{u} ; \delta \boldsymbol{\Lambda})-\bar{l}(\delta \boldsymbol{\Lambda})=0 & \\
\frac{\partial \mathcal{L}(\boldsymbol{z})}{\partial \lambda}=g_{j} & \leq 0, \quad j=1, \ldots, 2 n_{s}, & \text { for all } \delta \boldsymbol{\Lambda} \in \mathscr{U}^{0}, \\
\lambda_{j} & \geq 0, & j=1, \ldots, 2 n_{s}, \\
\lambda_{j} g_{j} & =0, & j=1, \ldots, 2 n_{s}, &
\end{array}
$$

where $\delta \boldsymbol{z} \in \mathscr{Z}^{0}=: \mathcal{S} \times \mathscr{U}^{0} \times \mathscr{U}^{0} \times \mathcal{S}^{2}$. Equation (36a) is the gradient equation, (36b) is the costate (or adjoint) equation, (36c)-(36d) are state relations for primal admissibility, (36e) is a state relation for dual admissibility, and (36f) is a complementary optimality condition. Note that (36c) is nothing else but the state problem (6).

For a second-order sufficient optimality condition for a stationary point of the Lagrange function (35), we refer to [Luenberger 1984].

Alternatively to the direct differentiation method in Section 4.2 the first derivative of the quantity of interest $q^{\prime}(\underline{s} ; \delta \underline{s})$ is derived by exploiting the adjoint equation (36b). Setting $\delta \boldsymbol{u}=\bar{\delta} \boldsymbol{u}$ in (36b) and using the result (29a) with the choice $\delta \boldsymbol{u}=\boldsymbol{\Lambda}$ we obtain

$$
Q_{\boldsymbol{u}}^{\prime}(\underline{s}, \boldsymbol{u} ; \bar{\delta} \boldsymbol{u})=-G_{\boldsymbol{u}}^{\prime}(\underline{s}, \boldsymbol{u} ; \boldsymbol{\Lambda}, \bar{\delta} \boldsymbol{u})=G_{\underline{s}}^{\prime}(\underline{s}, \boldsymbol{u} ; \boldsymbol{\Lambda}, \delta \underline{s}) \quad \text { for all } \delta \underline{s} \in \mathcal{S},
$$

where it is noteworthy to recall the relation $\bar{\delta} \boldsymbol{u}=\boldsymbol{u}_{\underline{s}}^{\prime}(\underline{s} ; \delta \underline{s})$. Inserting the result (37) into (29b), the required result for the first derivative of the quantity of interest by the adjoint state method becomes

$$
q^{\prime}(\underline{s} ; \delta \underline{s})=Q_{\underline{s}}^{\prime}(\underline{s}, \boldsymbol{u}(\underline{s}) ; \delta \underline{s})+G_{\underline{s}}^{\prime}(\underline{s}, \boldsymbol{u}(\underline{s}) ; \boldsymbol{\Lambda}, \delta \underline{s}) \quad \text { for all } \delta \underline{s} \in \mathcal{S} .
$$

Furthermore, by comparing (38) to (36a) we observe

$$
\mathcal{L}_{\underline{s}}^{\prime}(z ; \delta \underline{s})=q^{\prime}(\underline{s} ; \delta \underline{s})+\sum_{j=1}^{2 n_{s}} \lambda_{j} \nabla_{\underline{s}} g_{j}(\underline{s}) \cdot \delta \underline{s}=\underline{0} \quad \text { for all } \delta \underline{s} \in \mathcal{S} .
$$


For the case of simple constraints (34c) and (34d), the relation (39) can be simplified. Upon taking the derivatives of the inequalities (34c) and (34d), we obtain

$$
\begin{aligned}
g_{j} & =s_{j, \alpha_{k}}^{L}-s_{j} \Longrightarrow \frac{d g_{j}}{d s_{j}}=-1 \\
& \Rightarrow \lambda_{j} \nabla_{\underline{s}} g_{j}=\left[0, \ldots,-\lambda_{j}, \ldots, 0\right]^{T} \in \mathbb{R}^{n_{s}}, \quad j=1, \ldots, n_{s}, \\
g_{n_{s}+j} & =s_{j}-s_{j, \alpha_{k}}^{R} \Longrightarrow \frac{d g_{n_{s}+j}}{d s_{j}}=1 \\
& \Longrightarrow \lambda_{n_{s}+j} \nabla_{\underline{s}} g_{n_{s}+j}=\left[0, \ldots, \lambda_{n_{s}+j}, \ldots, 0\right]^{T} \in \mathbb{R}^{n_{s}}, \quad j=1, \ldots, n_{s} .
\end{aligned}
$$

Consequently, exploiting the Karush-Kuhn-Tucker conditions (36e)-(36f), as well as the relations (30), the necessary optimality condition (39) decomposes into the relations

$$
\{\nabla q(\underline{s})\}_{i}=\frac{\partial q}{\partial s_{i}}=\left\{\begin{aligned}
& 0 \text { if } s_{i, \alpha_{k}}^{L}<s_{i}<s_{i, \alpha_{k}}^{R}, \\
&-\lambda_{i} \nabla_{\underline{s}} g_{i} \underline{e}_{i}=\lambda_{i} \geq 0 \quad \text { if } s_{i}=s_{i, \alpha_{k}}^{L}, \\
&-\lambda_{n_{s}+i} \nabla_{\underline{s}} g_{n_{s}+i} \underline{e}_{i}=-\lambda_{n_{s}+i} \leq 0 \quad \text { if } s_{i}=s_{i, \alpha_{k}}^{R},
\end{aligned}\right.
$$

which are identical to the optimality conditions (31).

To summarize, under the assumptions that $\boldsymbol{u}$ is a unique solution of the state problem (6) for given design variables $\underline{s}$ and $(\underline{s}, \boldsymbol{u}) \in \mathcal{S} \times \mathscr{U}$ is a local solution of the optimization problem (34), there exist Lagrange multipliers $\boldsymbol{\Lambda} \in \mathscr{U}^{0}$ and $\underline{\lambda} \in \mathcal{S}^{2}$, such that the quadruple $(\underline{s}, \boldsymbol{u}, \boldsymbol{\Lambda}, \underline{\lambda})$ satisfies the Karush-Kuhn-Tucker conditions (36) with Lagrange functional defined in (35).

A second-order sensitivity analysis by means of the adjoint state differentiation method is performed in Appendix A.2.

4.4. Relation of least-squares problems to $\alpha$-level optimization. As pointed out in Section 2.2, the least-squares functional in (17) is typically used for parameter identification, thus minimizing the model error in (16) in a least-squares sense. The corresponding optimization problem reads

$$
\text { Find } q_{\mathrm{LS}}^{*}=\min _{\underline{s} \in \mathcal{S}} q_{\mathrm{LS}}(\underline{s})=\min _{\underline{s} \in \mathcal{S}} Q_{\mathrm{LS}}(\underline{s}, \boldsymbol{u}(\underline{s}))
$$

with $q_{\mathrm{LS}}$ according to (17). The solution vector of the least-squares problem shall be denoted by $s^{*}=\left[s_{1}^{*}, \ldots, s_{i}^{*}, \ldots s_{n_{p}}^{*}\right]^{T}$. In the context of parameter identification the design space is a parameter space $\mathcal{S} \subset \mathbb{R}^{n_{p}}$ of $n_{p}$ physically admissible material parameters. For example, using Young's modulus and Poisson's ratio as independent material parameters in linear elasticity we have $\underline{s}=[E v], n_{p}=2, \mathcal{S}_{1}=\mathbb{R}_{+}$, $\mathcal{S}_{2}=[0,0.5]$, and $\mathcal{S}=\mathcal{S}_{1} \times \mathcal{S}_{2} \subset \mathbb{R}^{2}$.

For the fuzzy analysis of the goal function $q_{\mathrm{LS}}$ we construct membership functions as triangular fuzzy numbers according to (21) by means of the least-squares 

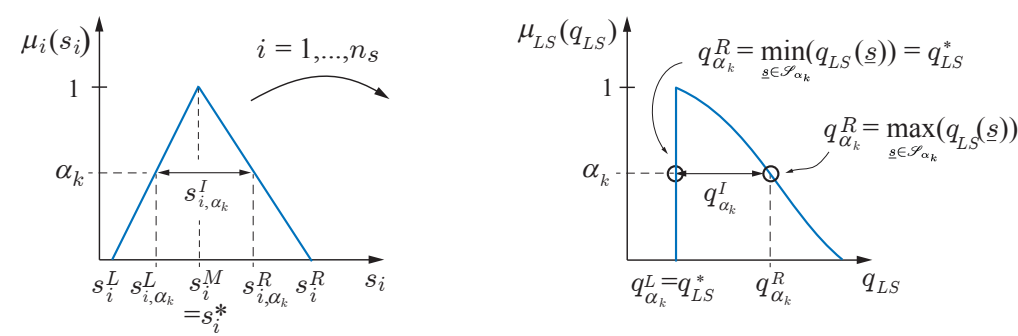

Figure 3. Schematic graph of $\alpha$-level optimization for leastsquares problems. Left: input membership function. Right: output membership function.

solutions $s_{i}^{*}$ as

$$
\begin{aligned}
\hat{A}_{i}=\left\langle s_{i}^{L}, s_{i}^{M}, s_{i}^{R}\right\rangle=\left\langle f^{L} s_{i}^{*}, s_{i}^{*}, f^{R} s_{i}^{*}\right\rangle, \\
\quad \text { where } f^{L}<1, f^{R}>1 \text {, and } i=1, \ldots, n_{s} .
\end{aligned}
$$

This is illustrated in Figure 3, left. For the corresponding $\alpha$-discretization we obtain lower and upper bounds of the crisp intervals $\mathcal{S}_{i, \alpha_{k}}=\left[s_{i, \alpha_{k}}^{L}, s_{i, \alpha_{k}}^{R}\right]$ at level $\alpha_{k}$ according to (23). With the corresponding input membership functions $\mu_{i}\left(s_{i}\right)$ it is the goal to determine the output membership function $\mu_{\mathrm{LS}}\left(q_{\mathrm{LS}}\right)$ of the quantity of interest $q_{\mathrm{LS}}$.

Since $s^{*}$ minimizes the least-squares problem (42), and since by construction of (43) we have $s_{i}^{*} \in \mathcal{S}_{i, \alpha_{k}}$, the minimum problems in (25) render the same solution $s^{*}$ to

$$
q_{\alpha_{k}}^{L}=\min _{\underline{s} \in \mathcal{S}_{\alpha_{k}}} q(\underline{s})=q_{\mathrm{LS}}^{*}=\min _{\underline{s} \in \mathcal{S}} q_{\mathrm{LS}}(\underline{s}) \quad \text { for all } 0 \leq \alpha_{k} \leq 1
$$

that is independent of the $\alpha$-level. Please note also that none of the constraints (23) in the minimization problems (44) are activated, that is, they are unconstrained. Consequently, the result (44) renders a vertical line for the left-hand part of the membership function in Figure 3, right. On the other hand, the maximization values

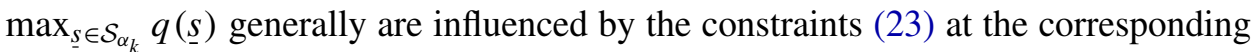
$\alpha$-levels, thus resulting in a decreasing part in the right-hand part of the membership function, which is schematically illustrated in Figure 3, right.

\section{Finite element discretization}

We introduce a finite element (FE) discretization as $\underline{s}_{h} \in \mathcal{S}_{h, \alpha_{k}} \subset \mathcal{S}_{\alpha_{k}}, \boldsymbol{u}_{h} \in \mathscr{U}_{h} \subset \mathscr{U}$, where $\mathcal{S}_{h, \alpha_{k}}$ and $\mathscr{U}_{h}$ are appropriate finite element spaces. In most cases, due to the finite dimension of $\mathcal{S}_{\alpha_{k}}$, the design variables $\underline{s}_{h}$ are in the same space as $\underline{s}$. An exception is the concept of hierarchical modeling; see, e.g., [Johansson et al. 2008]. 
The FE-discretized version of problem (25) is: find $\underline{s}_{h} \in \mathcal{S}_{\alpha_{k}}$ such that

$$
q\left(\underline{s}_{h}\right)=Q\left(\underline{s}_{h}, \boldsymbol{u}_{h}\left(\underline{s}_{h}\right)\right) \rightarrow \min _{\underline{s}_{h} \in \mathcal{S}_{h, \alpha_{k}}} \text { and } \quad G\left(\underline{s}_{h}, \boldsymbol{u}_{h}\left(\underline{s}_{h}\right)\right)=0 .
$$

In addition, with the discretization $\boldsymbol{\Lambda}_{h} \in \mathscr{U}_{h}^{0} \subset \mathscr{U}^{0}$ the FE-discretized version of the Lagrange function (35) is

$$
\mathcal{L}\left(\underline{s}_{h}, \boldsymbol{u}_{h}, \boldsymbol{\Lambda}_{h}, \underline{\lambda}_{h}\right):=Q\left(\underline{s}_{h}, \boldsymbol{u}_{h}\right)+G\left(\underline{s}_{h}, \boldsymbol{u}_{h} ; \boldsymbol{\Lambda}_{h}\right)+\sum_{i=1}^{2 n_{s}} \lambda_{h, j} g_{j}\left(\underline{s}_{h}\right) \underset{ }{\rightarrow} \operatorname{stat}_{z_{h} \in \mathscr{Z}_{h}},
$$

where $\left(\underline{s}_{h}, \boldsymbol{u}_{h}, \boldsymbol{\Lambda}_{h}, \underline{\lambda}_{h}\right)=: z_{h} \in \mathscr{Z}_{h}=: \mathcal{S}_{h} \times \mathscr{U}_{h} \times \mathscr{U}_{h}^{0} \times \mathcal{S}_{h}^{2}$ has been introduced. The quadruple $\left(\underline{s}_{h}, \boldsymbol{u}_{h}, \boldsymbol{\Lambda}_{h}, \underline{\lambda}_{h}\right)=: \boldsymbol{z}_{h}$ solves the discretized form of the optimization problem P2 in (34) provided a discretized form of the Karush-Kuhn-Tucker relations (36) is satisfied.

\section{Optimization methods}

Algorithms for solution of the optimization problems (25a) and (45) may be grouped into methods where only function evaluations are needed (zero-order methods) and where in addition its gradients are required (first-order methods), respectively. Another classification into deterministic and stochastic methods is possible. Examples of gradient-based deterministic methods are the Gauss-Newton method, the Levenberg-Marquard method, or the BFGS method; see, e.g., [Dennis and Schnabel 1983; Luenberger 1984]. An example of a gradient-free deterministic method is the simplex method; see, e.g., [Press et al. 1992]. Examples for stochastic methods are the Monte Carlo method, the evolution strategy, the method of Price and the method of Müller and Nollau; see, e.g., [Schwefel 1977], and [Möller et al. 2000] in the context of $\alpha$-level optimization.

Starting with an initialization $\underline{s}_{h}^{(0)}$, solution of the minimization problem (45) is achieved iteratively with a deterministic gradient-based strategy:

$$
\begin{aligned}
& \text { Projection algorithm [Bertsekas 1982]. } \\
& \underline{s}^{(j+1)}=\mathcal{P}\left\{\underline{s}^{(j)}-\alpha^{(j)} \underline{\bar{H}}^{(j)} \nabla q\left(\underline{s}^{(j)}\right)\right\} \\
& \text { where }(\mathcal{P}\{\underline{s}\})_{i}:=\min \left(s_{i, \alpha_{k}}^{L}, \max \left(s_{i}, s_{i, \alpha_{k}}^{R}\right)\right), i=1, \ldots, n_{s} .
\end{aligned}
$$

According to (23), $s_{i, \alpha_{k}}^{L}, s_{i, \alpha_{k}}^{R}$ are lower and upper bounds for the design variables. The iteration procedure (47) ensures a descent of the function value and feasibility of the parameters at each iteration step $j$. With the Jacobian $\underline{J}$ defined in (32b), 
three examples of iteration matrices at the $j$-th iteration step are

$$
\begin{aligned}
\text { Newton } & \underline{H}_{\mathrm{N}} & =\left[\nabla^{2} q(\underline{s})\right]^{-1}, \\
\text { Gauss-Newton } & \underline{H}_{\mathrm{GN}} & =\left[\underline{J}^{T} \underline{W}^{T} \underline{W} \underline{J}\right]^{-1}, \\
\text { Levenberg-Marquardt } & \underline{H}_{\mathrm{LM}} & =\left[\underline{J}^{T} \underline{W}^{T} \underline{W} \underline{J}+\gamma \boldsymbol{I}\right]^{-1}, \\
\text { BFGS } & \underline{H}_{\mathrm{BFGS}} & =\underline{H}\left(\underline{H}^{(j-1)}, \nabla q, \underline{s}\right) .
\end{aligned}
$$

The Newton method uses the full Hessian in (33), whereas the Gauss-Newton method neglects the matrix $\underline{M}$ in (33). It is not suitable if $\underline{J}$ does not have full rank. Then a Levenberg-Marquardt matrix becomes preferable, where various versions can be distinguished applying different strategies to choose the parameter $\gamma$; see, e.g., [Dennis and Schnabel 1983; Luenberger 1984]. Concerning the update formula for the BFGS algorithm we refer to [Luenberger 1984]. In order to preserve the descent property, a so-called diagonalization $\underline{H} \rightarrow \underline{\bar{H}}$ according to [Bertsekas 1982; Mahnken 1993] is employed. The line-search parameter $\alpha^{(j)}$ in the scheme (47) enlarges the area of convergence. An alternative globalization technique is trust-region; see, e.g., [Nocedal and Wright 1999].

Note that the above scheme (47) requires solution of the discrete version of the state problem (6). For nonlinear problems this is achieved within inner (equilibrium) iteration loops by means of the discrete counterpart of the Newton method in the equations (8). In addition, $n_{s}$ discrete versions of the tangent problem in (29a) have to be solved for calculation of the gradient $\nabla q$ in (32). To this end, the same (generally unsymmetric) linear tangent $G_{\boldsymbol{u}}^{\prime}(\cdot, \cdot ; \cdot, \cdot)$ as in (8), but with different right-hand sides, is used.

Gradient-based methods are able to determine only local minima, however are unable to overcome these. Therefore, different starting values should be applied for these types of methods. Stochastic methods can overcome local minima, however very often are not effective with respect to convergence. The advantages of both methods can be exploited in a hybrid strategy; see, e.g., [Quagliarella and Vicini 1998].

Similarly, solution of the discrete form of the Karush-Kuhn-Tucker equations (36) is obtained with the assembled vectors

$$
\tilde{\tilde{s}}=\left[\begin{array}{c}
\underline{s}_{h} \\
\boldsymbol{u}_{h} \\
\boldsymbol{\Lambda}_{h}
\end{array}\right], \quad \underline{r}=\left[\begin{array}{c}
\nabla_{s} \mathcal{L} \\
\nabla_{\boldsymbol{u}} \mathcal{L} \\
\nabla_{\boldsymbol{\Lambda}} \mathcal{L}
\end{array}\right] .
$$

Starting with an initialization $\underline{\tilde{s}}^{(0)}$, similarly to (50), the iteration scheme is now $\left.\underline{\tilde{s}}^{(j+1)}=\mathcal{P}\left\{\underline{\tilde{s}}^{(j)}-\alpha^{(j)} \underline{\bar{H}}^{(j)} \underline{r}^{(j)}\right)\right\}$,

$$
\text { where }(\mathcal{P}\{\underline{\tilde{s}}\})_{i}:= \begin{cases}\min \left(s_{i, \alpha_{k}}^{L}, \max \left(s_{i}, s_{i, \alpha_{k}}^{R}\right)\right) & \text { if } i=1, \ldots, n_{s}, \\ (\mathcal{P}\{\underline{\tilde{s}}\})_{i}:=\tilde{s}_{i} & \text { if } i=n_{s}+1, \ldots, \operatorname{dim}(\underline{\tilde{s}}),\end{cases}
$$



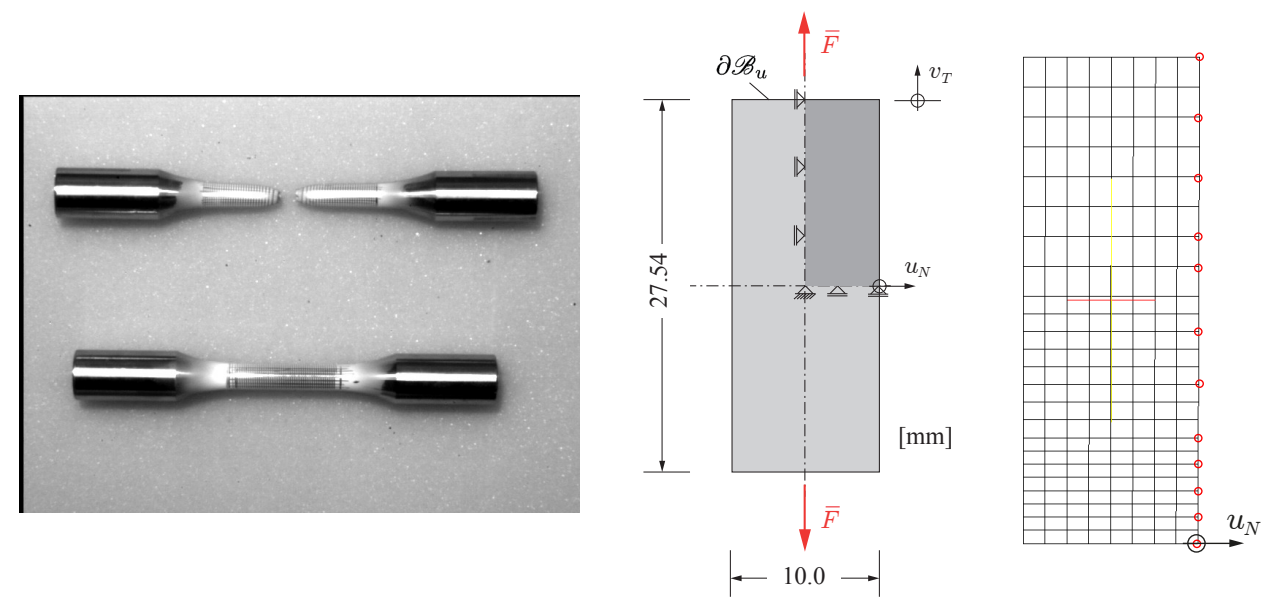

Figure 4. Axisymmetric necking. Left: photograph of failed and unused samples. Center: geometry. Right: discretization of a quarter with observation points.

until some convergence criterion is achieved. Contrary to the more conventional scheme (47), the iteration scheme (50) iterates simultaneously on all unknowns of the triple $\left(\underline{s}_{h}, \boldsymbol{u}_{h}, \boldsymbol{\Lambda}_{h}\right)$, thus avoiding inner (equilibrium) iteration loops. Note that the scheme (50) is an extension of the iterative scheme in [Johansson et al. 2007] for problems without simple constraints.

\section{Representative examples}

7.1. Axisymmetric necking at large strain elastoplasticity. In this example we consider the necking of a circular bar. The material of the specimen is a structural steel S355J2+N, according to EN 10025-2:200410 (formerly Baustahl St52-3 N, according to the German industrial code DIN 17100). In the photography in Figure 4, left, the sample after the experiment and a comparative sample are shown.

For experimental determination of the displacements, gratings are positioned on the surface. These are photographed with digital cameras at consecutive observation states NLST as listed in Table 1 in the displacement controlled experiment [Andresen et al. 1996]. The geometry under investigation is depicted in Figure 4, center. Here $v_{T}$ represents the total elongation at the different observation states with values according to Table 1 , and $\bar{F}$ is the resulting reaction force. Figure 4, right, shows a finite element discretization of a quarter model with selected observation points for parameter identification and the subsequent fuzzy analysis. Figure 5, top, depicts the grating at four observation states NLST $=5,7,10,13$ as introduced in Table 1. 


\begin{tabular}{|l|ccccc|}
\hline NLST & 1 & 3 & 4 & 5 & 6 \\
$v_{T}[\mathrm{~mm}]$ & $1.51 \cdot 10^{-3}$ & $1.2 \cdot 10^{-2}$ & $4.3 \cdot 10^{-2}$ & $1.1 \cdot 10^{-1}$ & $3.39 \cdot 10^{-1}$ \\
NLST & 7 & 8 & 10 & 13 & \\
$v_{T}[\mathrm{~mm}]$ & 3.24 & 3.83 & 4.61 & 5.47 & \\
\hline
\end{tabular}

Table 1. Axisymmetric necking: total elongation $v_{T}$ at 9 observation states.
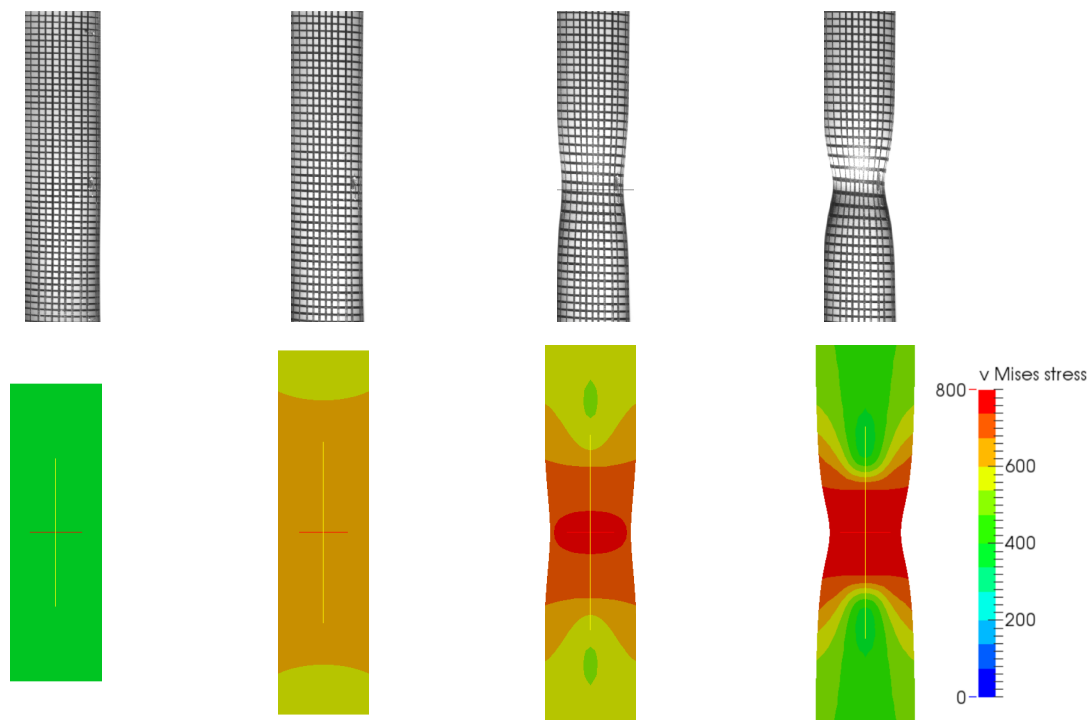

Figure 5. Axisymmetric necking. Top: photographs with a CCD camera of the sample and the grating at four different observation states NLST $=5,7,10,13$ according to Table 1 . Bottom: contours of von Mises stresses over the deformed configurations obtained from FE simulation.

The material is modeled by large strain multiplicative von Mises elastoplasticity according to

\begin{tabular}{|rl|}
\hline Kirchhoff stress & $\boldsymbol{\tau}=\mu \operatorname{dev}\left(\ln \boldsymbol{b}_{\mathrm{el}}\right)+K \ln J \boldsymbol{g}^{\sharp}$, \\
Yield function & $\Phi(\boldsymbol{\tau}, e ; \underline{\kappa})=\|\operatorname{dev}(\boldsymbol{\tau})\|-\sqrt{\frac{2}{3}} Y(e ; \underline{\kappa})$, \\
Flow stress & $Y(e ; \underline{\kappa})=Y_{0}+q(1-\exp (-b e))$, \\
Flow rule & $-\frac{1}{2} \mathcal{L}_{t}\left(\boldsymbol{b}_{\mathrm{el}}\right) \boldsymbol{b}_{\mathrm{el}}^{-1}=\gamma \frac{\operatorname{dev}(\boldsymbol{\tau})}{\|\operatorname{dev}(\boldsymbol{\tau})\|}$, \\
Variable evolution & $\dot{e}=\gamma \sqrt{\frac{2}{3}}$, \\
Loading and unloading conditions & $\gamma \geq 0, \Phi \leq 0, \gamma \Phi=0$. \\
\hline
\end{tabular}


Here, in addition to the notation in Section 2, we use $\tau=J \sigma$ as the Kirchhoff stress tensor with determinant $J>0$ of (4b), $\boldsymbol{b}_{\mathrm{el}}=\boldsymbol{F}_{\mathrm{el}} \boldsymbol{F}_{\mathrm{el}}^{T}$ as the elastic left Cauchy-Green tensor based on the multiplicative decomposition $\boldsymbol{F}=\boldsymbol{F}_{\mathrm{el}} \boldsymbol{F}_{\mathrm{pl}}$ for the deformation gradient in (4a), and $\boldsymbol{g}^{\sharp}$ as the contravariant metric tensor, and $\mathcal{L}_{t}(\cdot)={ }_{*} \Phi_{e}^{\sharp}\left[\partial_{t}^{*} \Phi_{e}^{\sharp}(\cdot)\right]$ denotes the Lie (time) derivative of a spatial argument with elastic pull-back and push-forward operators ${ }^{*} \Phi_{e}^{\sharp},{ }_{*} \Phi_{e}^{\sharp}$ according to (B-4) with $\boldsymbol{F}_{\mathrm{el}}$ replaced by $\boldsymbol{F}$; see, e.g., [Marsden and Hughes 1994; Simo and Miehe 1992; Mahnken 2005]. The vector of material parameters characterizing the inelastic material behavior is defined as

$$
\underline{s}=\left[Y_{0} b q\right]^{T} .
$$

Due to obvious symmetry conditions only a quarter of the specimen in Figure 4, right, is considered in the finite element simulation. An axisymmetric strain and stress state is assumed in the calculations. The mixed finite element formulation is based on a four-noded geometrically nonlinear enhanced strain method according to [Simo and Armero 1992]. A detailed analysis for the related sensitivity terms of the direct and the inverse analysis is provided in [Mahnken and Stein 1997].

The elastic constants are predetermined as $E=20600 \mathrm{kN} / \mathrm{cm}^{2}$ for Young's modulus and $v=0.3$ for Poisson's ratio. The additional 3 material parameters in (52) characterizing the inelastic material behavior are obtained by minimizing the least-squares functional

$$
q_{\mathrm{LS}}(\underline{s})=Q_{\mathrm{LS}}(\boldsymbol{u}(\underline{s}))=\sum_{i=1}^{n_{\mathrm{tdat}}} \sum_{j=1}^{n_{\mathrm{mp}}}\left\|\underline{\boldsymbol{u}}_{i j}(\underline{s})-\underline{\bar{u}}_{i j}\right\|+\sum_{i=1}^{n_{\mathrm{tdat}}}\left(w\left(F_{i}(\underline{s})-\bar{F}_{i}\right)\right) .
$$

Here $\underline{\boldsymbol{u}}_{i j}=\left[\bar{u}_{x}, \bar{u}_{y}\right]_{i j}$ and $\bar{F}_{i}$ assemble experimental data for displacements at $n_{\mathrm{mp}}$ observation points and reaction forces, respectively, for $n_{\text {tdat }}$ observation states. Analogously, $\underline{\boldsymbol{u}}_{i j}=\left[u_{x}, u_{y}\right]_{i j}$ assembles simulated data for displacements and $F_{i}(\underline{s})$ denotes reaction forces, analogously to the quantity of interest in (10), i.e.,

$$
F_{i}(\boldsymbol{u})=\int_{\partial \mathscr{B}_{u}}\left\|\boldsymbol{P}\left(\boldsymbol{u}_{i}\right)^{T} \cdot \boldsymbol{N}\right\|_{2} d A, \quad i=1, \ldots, n_{\text {tdat }}
$$

To be more specific, the weighting factor in (53) is chosen as $w=\sqrt{10^{-3}}$, the number of load steps is $N=40$, the number of observation states is $n_{\text {tdat }}=9$ according to Table 1 , and there are $n_{\mathrm{mp}}=12$ observation points. In this way, the functional (53) represents a special case of (17) with the quantity of interest for the model error by taking into account both displacements and reaction forces with adequate weighting. 

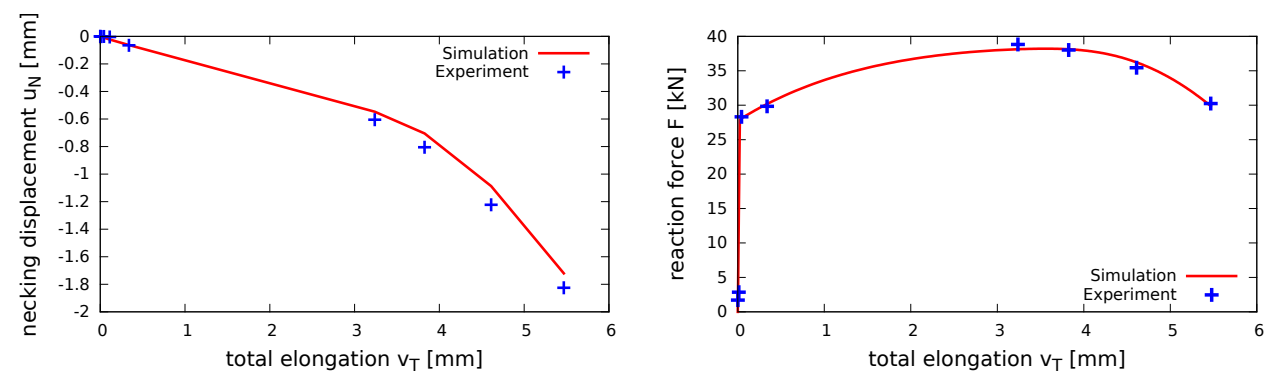

Figure 6. Axisymmetric necking: comparison of simulation and experiment. Left: necking displacement versus loading. Right: reaction force versus loading.
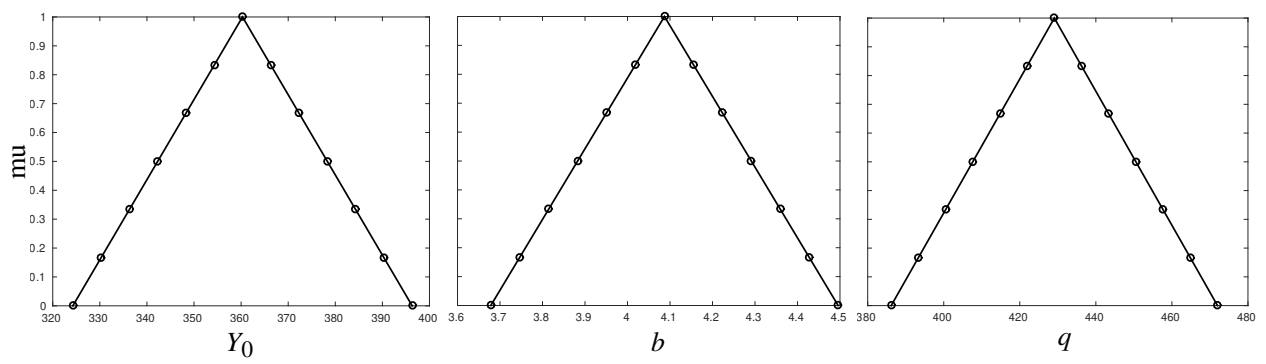

Figure 7. Axisymmetric necking: input membership functions for the material parameters $Y_{0}, b, q$.

The resulting vector for the material parameters after optimization is

$$
\underline{s}^{*}=\left[\begin{array}{c}
Y_{0} \\
b \\
q
\end{array}\right]=\left[\begin{array}{c}
360.30 \mathrm{~N} / \mathrm{mm}^{2} \\
4.0869 \\
429.08 \mathrm{~N} / \mathrm{mm}^{2}
\end{array}\right] .
$$

Figure 5, bottom, depicts the von Mises stress over the deformed configurations. The nonuniformness with increasing necking behavior becomes striking. In Figure 6, left and right, the maximal necking displacement $u_{N}$, introduced in Figure 4, center, and the total load $F$, defined in (54), respectively, versus the total elongation $v_{T}$ are compared for FE simulation and experimentation, thus illustrating the excellent agreement for both quantities.

Based on the above results of parameter identification, the following considerations are directed to the fuzzy analysis of the material parameters obtained in (55). To this end, firstly membership functions are generated according to Figure 7 as input quantities for the fuzzy analysis. The parameters $Y_{0}, b, q$ in (52) are chosen as mean values to obtain triangular fuzzy numbers according to (44) as

$$
\hat{A}_{i}=\left\langle s_{i}^{L}, s_{i}^{M}, s_{i}^{R}\right\rangle=\left\langle 0.9 s_{i}^{*}, s_{i}^{*}, 1.1 s_{i}^{*}\right\rangle,
$$



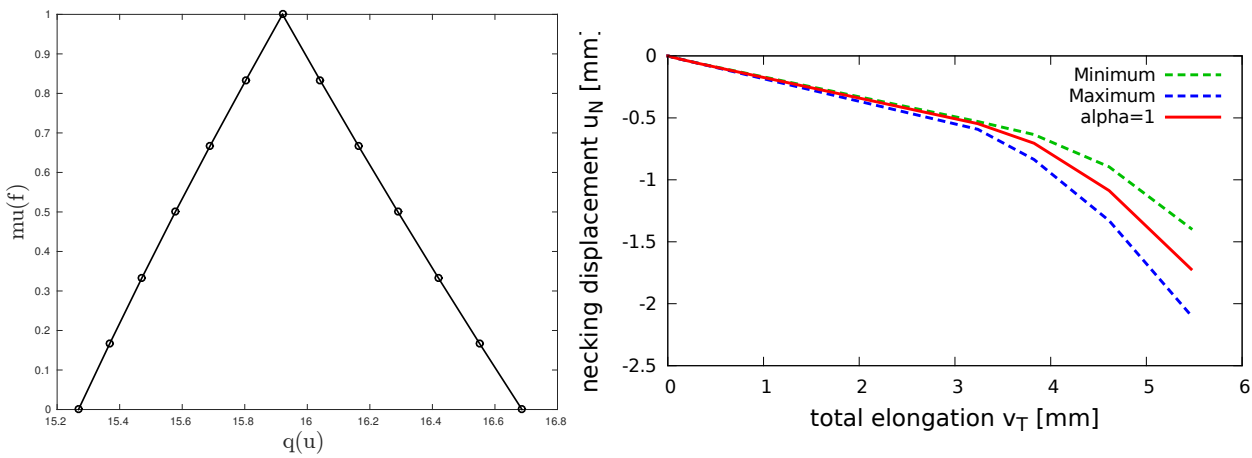

Figure 8. Axisymmetric necking: fuzzy results for quantity of interest with displacements. Left: output membership function. Right: uncertainty regime.
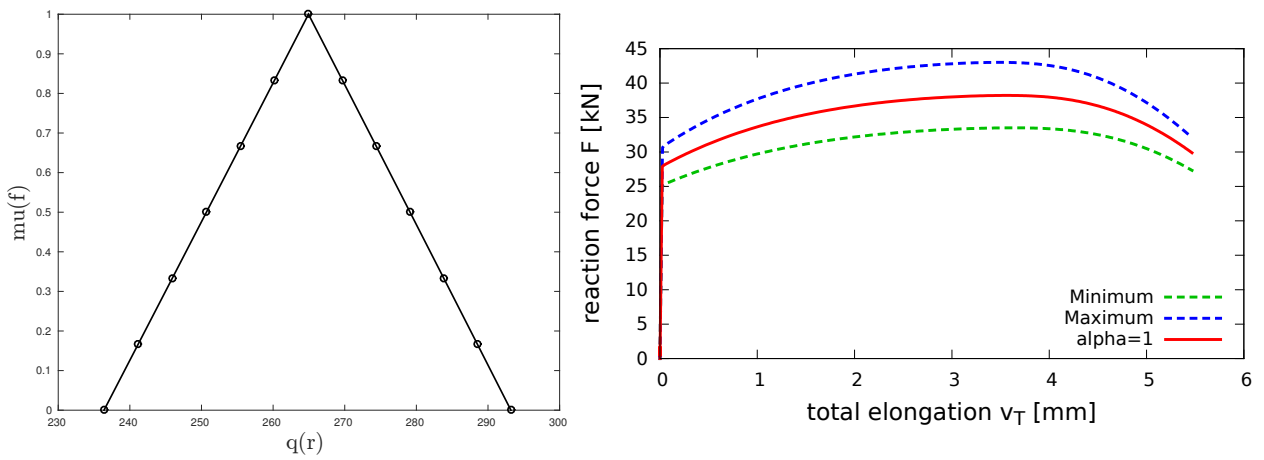

Figure 9. Axisymmetric necking: fuzzy results for quantity of interest with reaction forces. Left: output membership function. Right: uncertainty regime.

that is, for each material parameter a maximum deviation of $10 \%$ for the uncertainty is assumed.

The $\alpha$-discretization is performed with 7 equally spaced intervals. In the fuzzy analysis we are interested in the uncertainties of the quantities of interest

$$
\begin{aligned}
& q_{u}(\underline{s}):=Q(\boldsymbol{u}(\underline{s})):=\sum_{i=1}^{n_{\text {tdat }}} \sum_{j=1}^{n_{\mathrm{mp}}}\left\|\underline{\boldsymbol{u}}_{i j}(\underline{s})\right\|, \\
& q_{F}(\underline{s}):=Q\left(F_{i}(\underline{s})\right):=\sum_{i=1}^{n_{\text {tdat }}} F_{i}(\underline{s})
\end{aligned}
$$

with displacements $\underline{\boldsymbol{u}}_{i j}(\underline{s})$ according to (53) and reaction forces $F_{i}(\underline{s})$ according to (54). As a third quantity we choose the model error functional $q_{\mathrm{LS}}(\underline{s})$ in (53). 

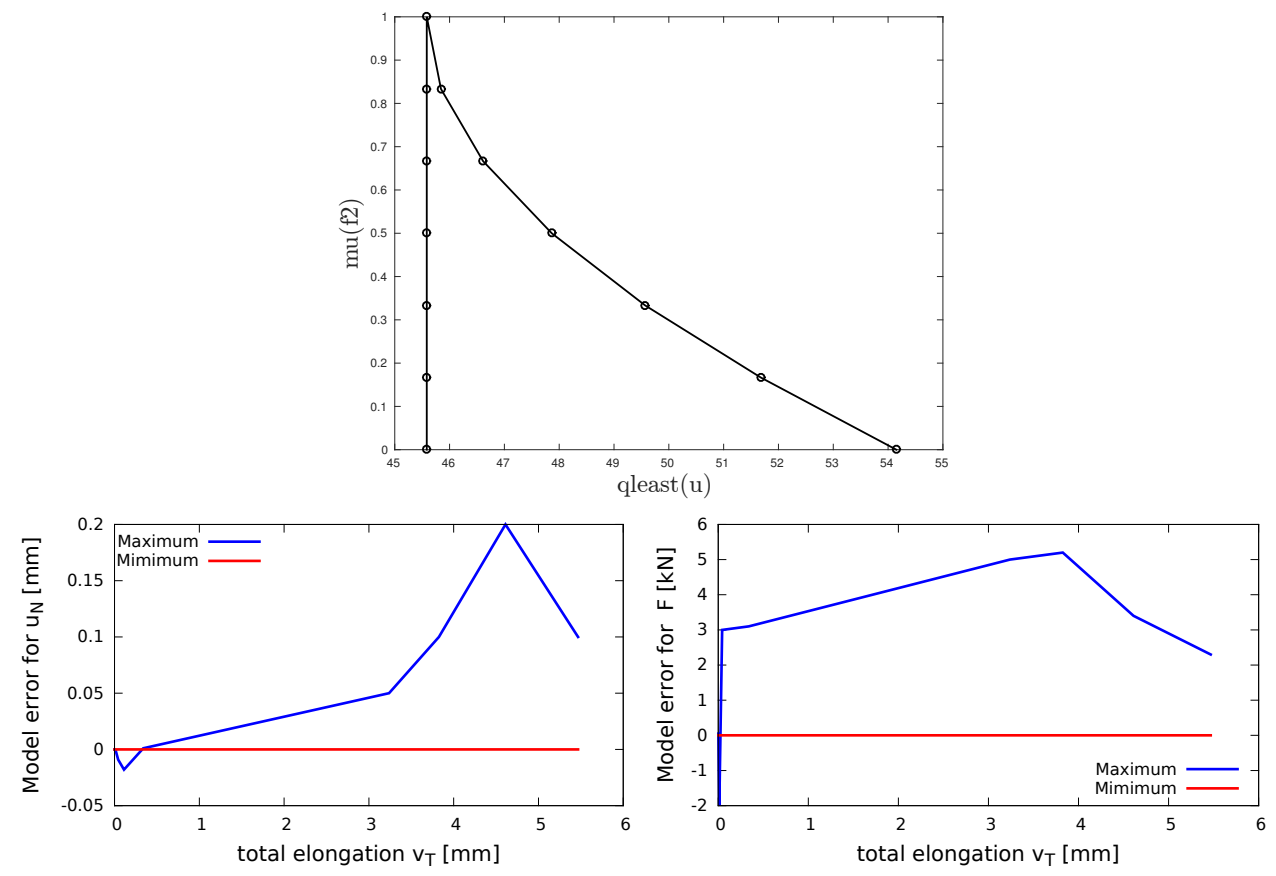

Figure 10. Axisymmetric necking: fuzzy results for quantity of interest with model error. Top: output membership function. Bottom left: uncertainty regime for model of necking displacement. Bottom right: uncertainty regime for model of reaction force.

For all three quantities of interest the minimization and maximization problems P1a and P1b, respectively, according to (25) are solved at each $\alpha$-level.

Figures 8 and 9 , left, show the output membership functions $\mu\left(q_{u}\right)$ and $\mu\left(q_{F}\right)$, respectively, for the quantity of interest with displacements in (57a) and the quantity of interest with reaction forces in (57b). Remarkably, both output membership functions exhibit an almost linear behavior, although the underlying geometrical and physical behavior is highly nonlinear. The corresponding Figures 8 and 9, right, display the uncertainty regimes for the necking displacement $u_{N}$ and the reaction force $\bar{F}$.

Figure 10 summarizes the fuzzy results for the quantity of interest formulated as a model error based on the least-squares functional in (53). Figure 10, top, shows the output membership functions $\mu\left(q_{\mathrm{LS}}\right)$. Note that the minimum problems $\min _{s \in \mathcal{S}_{\alpha_{k}}} q(s)$ in (25) of all $\alpha$-level optimization coincide with the minimization problems for the least-squares functional in (53). As explained in Section 4.4, the constraints (23) are not activated at the $\alpha$-levels, thus resulting in a vertical line in the left-hand side of the membership function in Figure 10, top. On the other 
hand, the maximization values $\max _{s \in \mathcal{S}_{\alpha_{k}}} q(s)$ exhibit a strong nonlinear behavior in Figure 10, top, influenced by the constraints (23) at the corresponding $\alpha$-levels. Figure 10, bottom left and right, display the related uncertainty regimes for the model errors of necking displacement and reaction force, respectively. Note that in both diagrams the curves obtained from the minimum problems coincide with those of $\alpha=1$.

The maximum deviations from the quantities at $\alpha=1$ for all three quantities are

$$
\begin{aligned}
& \Delta_{u}^{L}=\frac{q_{u \alpha=0}^{L}-q_{u \alpha=1}^{L}}{q_{u \alpha=1}^{L}} 100=4.10 \%, \quad \Delta_{u}^{R}=\frac{q_{u \alpha=0}^{R}-q_{u \alpha=1}^{L}}{q_{u \alpha=1}^{L}} 100=4.81 \%, \\
& \Delta_{F}^{L}=\frac{q_{F \alpha=0}^{L}-q_{F \alpha=1}^{L}}{q_{F \alpha=1}^{L}} 100=10.77 \%, \Delta_{F}^{R}=\frac{q_{F \alpha=0}^{R}-q_{F \alpha=1}^{L}}{q_{F \alpha=1}^{L}} 100=10.68 \% \text {, } \\
& \Delta_{\mathrm{LS}}^{L}=\frac{q_{\mathrm{LS} \alpha=0}^{L}-q_{\mathrm{LS} \alpha=1}^{L}}{q_{\mathrm{LS} \alpha=1}^{L}} 100=0 \%, \quad \Delta_{\mathrm{LS}}^{R}=\frac{q_{\mathrm{LS} \alpha=0}^{R}-q_{\mathrm{LS} \alpha=1}^{L}}{q_{\mathrm{LS} \alpha=1}^{L}} 100=18.79 \%,
\end{aligned}
$$

thus revealing a larger uncertainty for the reaction forces as for the displacements.

7.2. Perforated strip at large strain elasticity. In the second representative example measurements are obtained from an experiment for a polyurethane sample. The experimental setting is visualized in Figure 11, top left. The geometry and dimensions of the specimen are shown in Figure 11, top center, where $t=2 \mathrm{~mm}$ is its thickness. Measurements for the parameter identification are taken at prescribed displacements $v_{T}$ according to Table 2 . The values for $\bar{F}$ in Table 2 represent the measured total loads at the different observation states. Displacement fields are obtained by noncontacting optical measurements, which require a speckle pattern on the surface of the specimen as shown in Figure 11, top right. The observation points, where measurements are available, are highlighted in Figure 11, bottom.

The material is modeled in large strain elasticity with an the Ogden model according to [Ogden 1997]. This hyperelastic material model is based on principal stretches with a strain-energy function for the isochoric part given by

$$
\hat{\psi}(\boldsymbol{u}, \underline{s})=\sum_{A=1}^{3} \sum_{\alpha=1}^{n} \frac{c_{\alpha}}{m_{\alpha}}\left(\hat{\lambda}_{A}^{m_{\alpha}}-1\right) .
$$

The deviatoric principal stretches are given by $\hat{\lambda}_{A}=J^{-1 / 3} \lambda_{A}$ with principal stretches $\lambda_{A}$, obtained from a spectral decomposition of the right Cauchy-Green tensor $C=\sum_{A=1}^{3} \lambda_{A}^{2} N_{A} \otimes N_{A}$. The vector of material parameters is defined as

$$
\underline{s}=\left[c_{1}, m_{1}, \ldots, c_{n}, m_{n}\right]^{T} .
$$



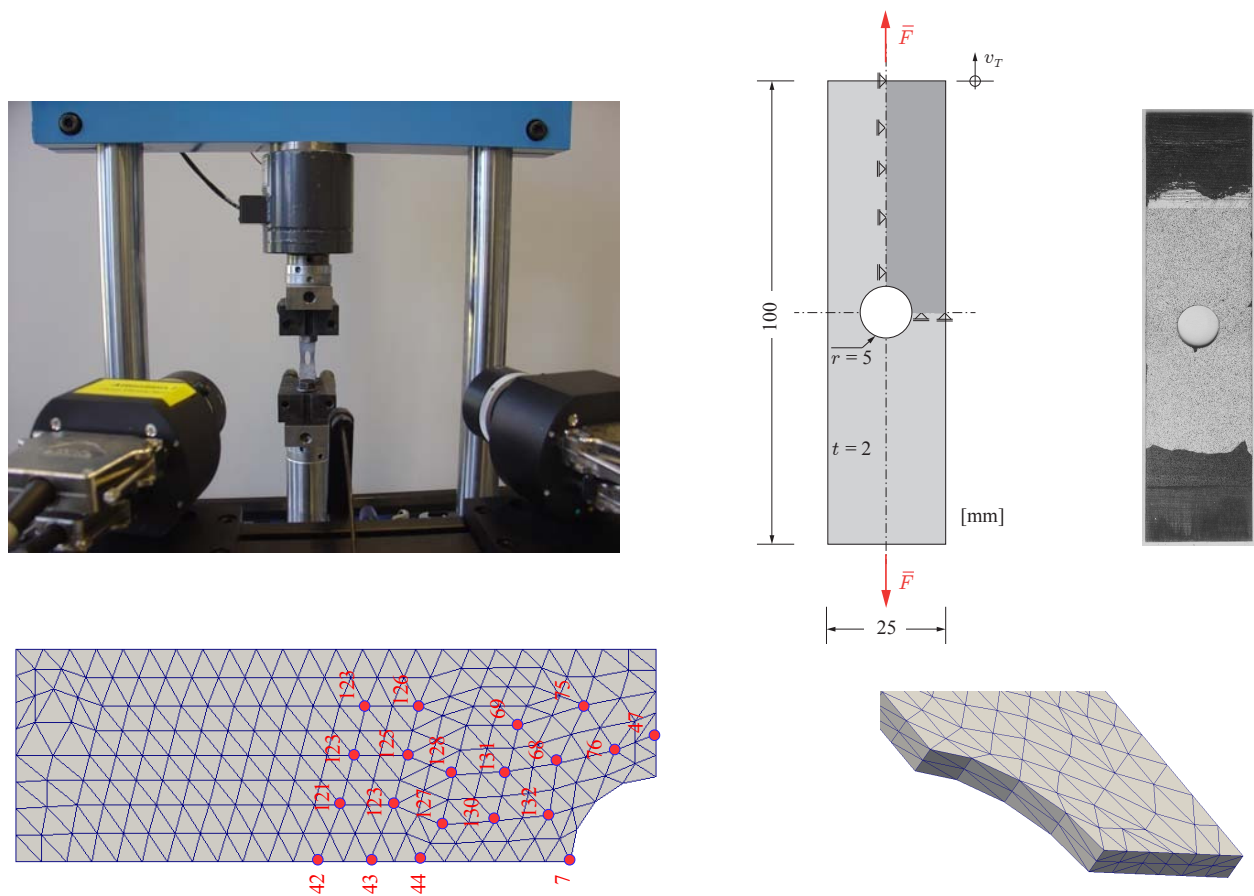

Figure 11. Perforated strip. Top left: experimental setup. Top center: geometry. Top right: speckle pattern. Bottom: threedimensional discretization of a quarter with observation points.

\begin{tabular}{|l|lllll|}
\hline NLST & 6 & 12 & 18 & 24 & 30 \\
$v_{T}[\mathrm{~mm}]$ & 6 & 12 & 18 & 24 & 30 \\
$\bar{F}[\mathrm{~N}]$ & 10.88 & 17.23 & 21.33 & 24.31 & 26.53 \\
\hline
\end{tabular}

Table 2. Perforated strip: total load $\bar{F}$ at 5 observation states.

Closed-form expressions for stresses and tangent moduli in terms of the reference configuration as well as the current configuration have been derived in [Simo and Taylor 1991].

Due to obvious symmetry conditions only a quarter of the specimen in Figure 11, bottom, is considered in the finite element simulation. The mixed finite element formulation is based on a four-noded geometrically nonlinear enhanced strain method for tetrahedra according to [Caylak and Mahnken 2012]. A detailed analysis for the related sensitivity terms of the direct and inverse analyses is provided in [Mahnken and Stein 1997].

Hereafter, we set $n=1$ for the Ogden model in (60); that is, $c_{1}$ and $m_{1}$ are the required material parameters. The finite element simulation is force-driven 


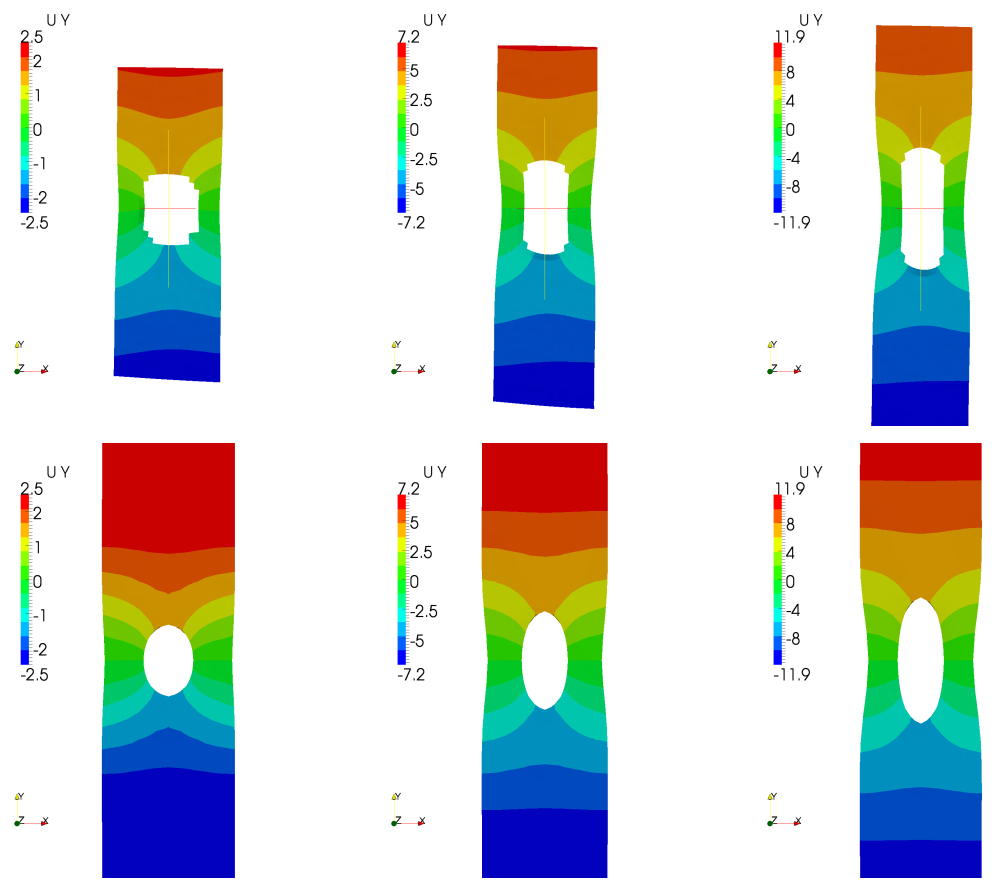

Figure 12. Perforated strip: contour plot of displacement in vertical direction. Top: experiments. Bottom: simulations. Left: NLST 6. Center: NLST 18. Right: NLST 30.

with the values according to Table 2 . In this way it is possible to incorporate the measured force into the parameter identification and make it more robust with respect to nonsymmetric fixation in longitudinal direction. The following leastsquares functional is introduced:

$$
q_{\mathrm{LS}}(\underline{s})=Q(\boldsymbol{u}(\underline{s}))=\sum_{i=1}^{n_{\text {tdat }}} \sum_{j=1}^{n_{\mathrm{mp}}}\left\|\underline{\boldsymbol{u}}_{i j}(\underline{s})-\underline{\overline{\boldsymbol{u}}}_{i j}\right\| .
$$

Here $\overline{\boldsymbol{u}}_{i j}=\left[\bar{u}_{x}, \bar{u}_{y}\right]_{i j}$ assembles experimental data for displacements at $n_{\mathrm{mp}}$ observation points for $n_{\text {tdat }}$ observation states, and $\underline{\boldsymbol{u}}_{i j}=\left[u_{x}, u_{y}\right]_{i j}$ assembles the corresponding simulated data. The number of load steps is $N=15$, the number of observation states according to Table 2 is $n_{\text {tdat }}=5$, and there are $n_{\mathrm{mp}}=35$ observation points. In this way the functional (61) represents a special case of (17) with the quantity of interest for the model error. The resulting vector for the material parameters after optimization is

$$
\underline{s}^{*}=\left[\begin{array}{c}
c_{1} \\
m_{1}
\end{array}\right]=\left[\begin{array}{r}
8.0997 \\
-1.9360
\end{array}\right] \text {. }
$$



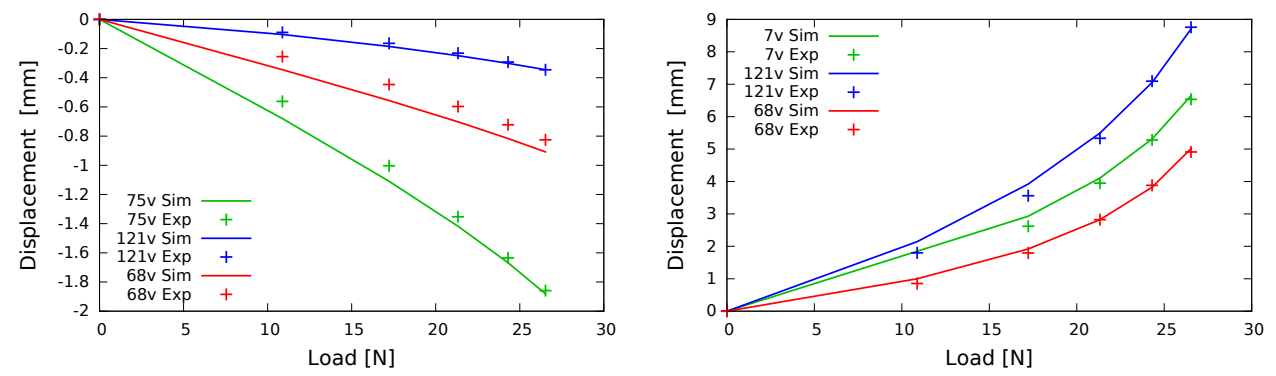

Figure 13. Perforated strip: displacements for experiment and simulation at different observation points. Left: horizontal displacement $u_{x}$ versus loading. Right: vertical displacement $u_{y}$ versus loading.
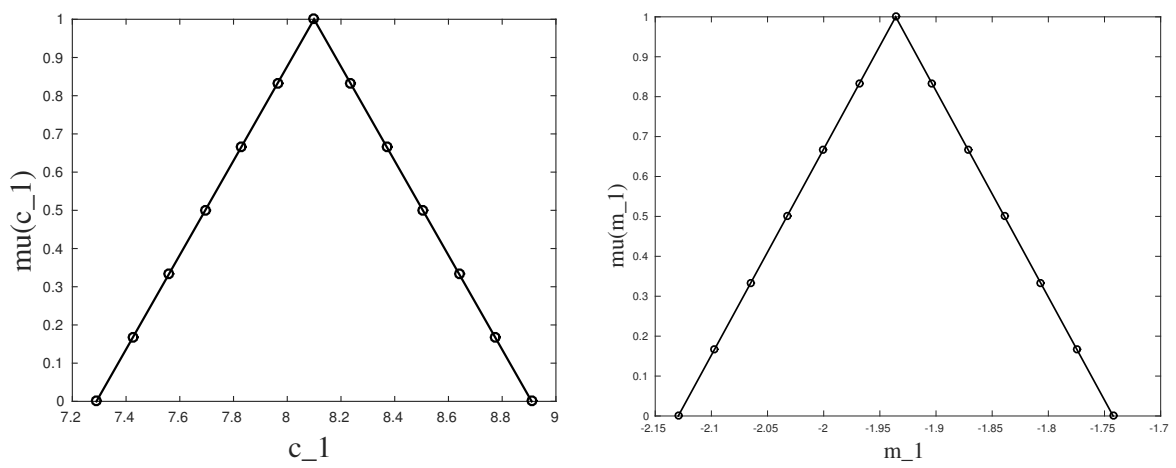

Figure 14. Perforated strip: input membership functions for material parameters. Left: parameter $c_{1}$. Right: parameter $m_{1}$.

For three of these steps, NLST $=6,18,30$, the measured displacement in the vertical direction on the surface of the specimen is presented in the first row of Figure 12. Please note that the colored area does not cover the whole surface of the specimen due to limitations of the measurement technology. However, the agreement to the results of simulation in the second row of Figure 12 is well established.

For some observation nodes the horizontal as well as the vertical displacements $u_{x}$ and $u_{y}$ are plotted over the load in Figure 13, left and right, respectively. The dots are the measured values, and the lines are the computed values. A very reasonable agreement is obtained for both types of quantities.

Based on the above results of parameter identification the following considerations are directed to the fuzzy analysis of the material parameters obtained in (62). To this end, firstly membership functions are generated according to Figure 14, as input quantities for the fuzzy analysis. The parameters in (60) are chosen as mean 

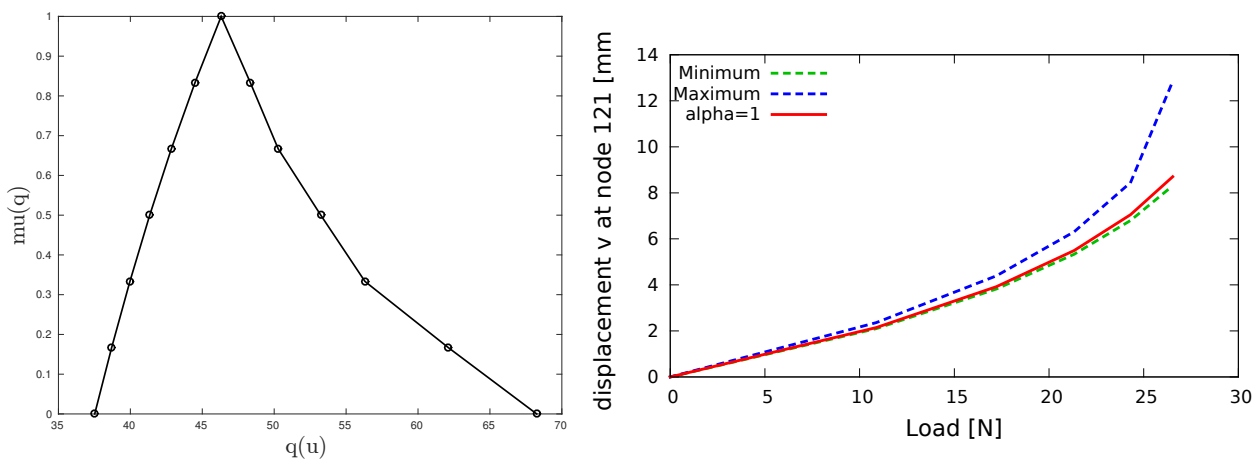

Figure 15. Perforated strip: fuzzy results for quantity of interest with displacements. Left: output membership function. Right: uncertainty regime for vertical displacement at observation point 121.

values to obtain triangular fuzzy numbers according to (44) as

$$
\hat{A}_{i}=\left\langle s_{i}^{L}, s_{i}^{M}, s_{i}^{R}\right\rangle=\left\langle 0.9 s_{i}^{*}, s_{i}^{*}, 1.1 s_{i}^{*}\right\rangle
$$

that is, for each material parameter a maximum deviation of $10 \%$ for the uncertainty is assumed. The $\alpha$-discretization is performed with 7 equally spaced intervals. In the fuzzy analysis we are firstly interested in the uncertainty of the quantity of interest

$$
q_{u}(\underline{s}):=Q(\boldsymbol{u}(\underline{s}))=\sum_{i=1}^{n_{\text {tdat }}} \sum_{j=1}^{n_{\mathrm{mp}}}\left\|\underline{\boldsymbol{u}}_{i j}(\underline{s})\right\|,
$$

with displacements $\underline{\boldsymbol{u}}_{i j}(\underline{s})$ according to (61). As a second quantity we choose the least-squares functional $q_{\mathrm{LS}}(\underline{s})$ in (61). For both quantities of interest the minimization and maximization problems P1a and P1b, respectively, according to (25) are solved at each $\alpha$-level.

Figure 15, left, shows the output membership functions $\mu\left(q_{u}\right)$ for the quantity of interest with displacements in (64). The corresponding Figure 15, right, displays the uncertainty regime for the vertical displacement $v$ at a specific observation point 121 displayed in Figure 11.

Figure 16 summarizes the fuzzy results for the quantity of interest formulated as a model error based on the least-squares functional in (64). Figure 16, left, shows the output membership functions $\mu_{\mathrm{LS}}\left(q_{\mathrm{LS}}\right)$. As explained in Section 4.4, the constraints (23) are not activated at the $\alpha$-levels, thus resulting in a vertical line in the left-hand side of the membership function in Figure 16, left. On the other hand, the maximization values $\max _{s \in \mathcal{S}_{\alpha_{k}}} q(s)$ exhibit a strong nonlinear behavior in Figure 16, left, influenced by the constraints (23) at the corresponding $\alpha$-level. 

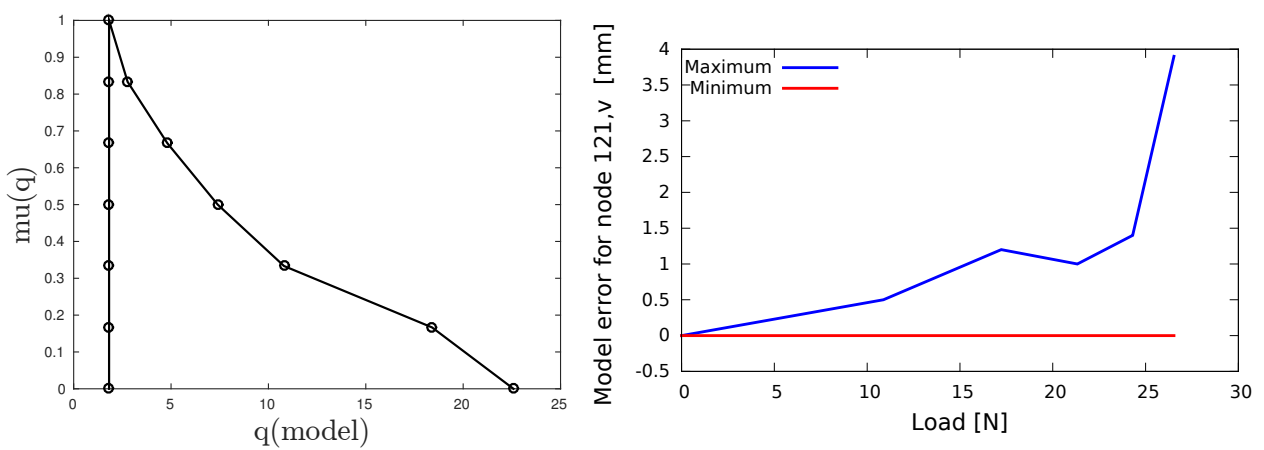

Figure 16. Perforated strip: fuzzy results for quantity of interest with model error. Left: output membership function. Right: uncertainty regime for model error at observation point 121 .

Figure 16, right, displays the related uncertainty regimes for the model errors of the displacement at the observation point 121.

The maximum deviations from the quantities at $\alpha=1$ for all three quantities are

$$
\begin{array}{cc}
\Delta_{u}^{L}=\frac{q_{u \alpha=0}^{L}-q_{u \alpha=1}^{L}}{q_{u \alpha=1}^{L}} 100=19.00 \%, & \Delta_{u}^{R}=\frac{q_{u \alpha=0}^{R}-q_{u \alpha=1}^{L}}{q_{u \alpha=1}^{L}} 100=47.36 \%, \\
\Delta_{\mathrm{ME}}^{L}=\frac{q_{\mathrm{LS} \alpha=0}^{L}-q_{\mathrm{LS} \alpha=1}^{L}}{q_{\mathrm{LS} \alpha=1}^{L}} 100=0 \%, & \Delta_{\mathrm{ME}}^{R}=\frac{q_{\mathrm{LS} \alpha=0}^{R}-q_{\mathrm{LS} \alpha=1}^{L}}{q_{\mathrm{LS} \alpha=1}^{L}} 100=1142 \%,
\end{array}
$$

thus revealing a comparatively large uncertainty especially for the model error.

\section{Conclusion}

This work is focused on epistemic uncertainties in the framework of continuum mechanics, which is taken into account with fuzzy analysis. The underlying min-max optimization problem of the extension principle is approximated by $\alpha$-discretization, resulting in a separation of minimum and maximum problems. To become more general, so-called quantities of interest are employed, which allow a general formulation for the target problem of interest.

Variational formulations have at least two intrinsic advantages.

- They enable a general framework for the analytical sensitivity analysis, which allows the effective solution of the related optimization problems with gradientbased methods.

- They give the basis for adaptive refinement of the underlying FE discretization. 
The relation to parameter identification problems based on least-squares functions is highlighted. In particular we have shown that the solution of the leastsquares problem coincides with the solution of the related minimization problems at all $\alpha$-levels.

The solutions of the related optimization problems with simple constraints are obtained with a gradient-based scheme, which is derived from a sensitivity analysis for the target problem. Two numerical examples for the fuzzy analysis of material parameters are concerned with a necking problem at large strain elastoplasticity and a perforated strip at large strain hyperelasticity. Further research will be directed to the following topics.

- Future investigations could be focused on different kinds of design variables, in addition to material parameters considered in the examples of this work.

- The adoption of adaptive mesh refinement which is driven by a goal quantity of interest results in more accurate results at fewer degrees of freedom and therefore is of great importance [Widany and Mahnken 2012].

- In most simulations uncertainties of different types occur. Therefore, the type of epistemic uncertainty should be combined with the type of aleatoric uncertainty in a polymorphic uncertainty model; see, e.g., [Graf et al. 2015].

- The present article is focused on the case of simple constraints. Therefore, the interaction of the fuzzy variables is a further challenge in future work on fuzzy analysis [Möller and Beer 2004].

\section{Appendix A: Second-order sensitivity analysis}

This appendix provides the second-order sensitivity analysis complementary to the first-order sensitivity analysis in Section 4.

A.1. The direct differentiation method. We begin the second-order analysis by the direct differentiation method analogously to the first-order analysis for derivation of (29). To this end, we apply the chain rule to the reduced functionals in (29a) and $(29 b)$ :

$$
\begin{aligned}
g^{\prime \prime}\left(\underline{s} ; \delta \underline{s}_{1}, \delta \underline{s}_{2}\right)= & G_{\underline{s}}^{\prime \prime}\left(\underline{s}, \boldsymbol{u}(\underline{s}) ; \delta \boldsymbol{u}, \delta \underline{s}_{1}, \delta \underline{s}_{2}\right)+G_{\boldsymbol{u} \underline{s}}^{\prime \prime}\left(\underline{s}, \boldsymbol{u}(\underline{s}) ; \delta \boldsymbol{u}, \bar{\delta} \boldsymbol{u}_{1}, \delta \underline{s}_{2}\right) \\
& +G_{\underline{s} \boldsymbol{u}}^{\prime \prime}\left(\underline{s}, \boldsymbol{u}(\underline{s}) ; \delta \boldsymbol{u}, \delta \underline{s}_{1}, \bar{\delta} \boldsymbol{u}_{2}\right)+G_{\boldsymbol{u} \boldsymbol{u}}^{\prime \prime}\left(\underline{s}, \boldsymbol{u}(\underline{s}) ; \delta \boldsymbol{u}, \bar{\delta} \boldsymbol{u}_{1}, \bar{\delta} \boldsymbol{u}_{2}\right) \\
& +G_{\boldsymbol{u}}^{\prime}\left(\underline{s}, \boldsymbol{u}(\underline{s}) ; \delta \boldsymbol{u}, \bar{\delta}^{2} \boldsymbol{u}\right)=0 \\
& \quad \text { for all } \delta \boldsymbol{u} \in \mathscr{U}^{0} \text { and } \delta \underline{s}_{1}, \delta \underline{s}_{2} \in \mathcal{S}_{\alpha_{k}}, \quad(A) \\
q^{\prime \prime}\left(\underline{s} ; \delta \underline{s}_{1}, \delta \underline{s}_{2}\right)= & Q_{\underline{s} \underline{s}}^{\prime \prime}\left(\underline{s}, \boldsymbol{u}(\underline{s}) ; \delta \underline{s}_{1}, \delta \underline{s}_{2}\right)+Q_{\boldsymbol{u} \underline{s}}^{\prime \prime}\left(\underline{s}, \boldsymbol{u}(\underline{s}) ; \bar{\delta} \boldsymbol{u}_{1}, \delta \underline{s}_{2}\right) \\
& +Q_{\underline{s} \boldsymbol{u}}^{\prime \prime}\left(\underline{s}, \boldsymbol{u}(\underline{s}) ; \delta \underline{s}_{1}, \bar{\delta} \boldsymbol{u}_{2}\right)+Q_{u \boldsymbol{u}}^{\prime \prime}\left(\underline{s}, \boldsymbol{u}(\underline{s}) ; \bar{\delta} \boldsymbol{u}_{1}, \bar{\delta} \boldsymbol{u}_{2}\right) \\
& +Q_{u}^{\prime}\left(\underline{s}, \boldsymbol{u}(\underline{s}) ; \bar{\delta}^{2} \boldsymbol{u}\right) \text { for all } \delta \underline{s}_{1}, \delta \underline{s}_{2} \in \mathcal{S}_{\alpha_{k}},
\end{aligned}
$$


where analogously to (29) $G_{s s}^{\prime \prime}(\cdot, \cdot ; \cdot ; \cdot), G_{u s}^{\prime \prime}(\cdot, \cdot ; \cdot, \cdot, \cdot)$, etc., denote partial derivatives with respect to $\underline{s}$ and $\boldsymbol{u}$, defined as Gâteaux derivatives. Equation (A-1a) is zero since $\boldsymbol{u}(\underline{s})$ solves the tangent problem (29a) for each $\underline{s} \in \mathcal{S}$ [Johansson et al. 2007; Mahnken and Stein 1996] in the context of homogeneous deformation problems. Equation (A-1b) follows by differentiation of (29b). Inserting the solution $\bar{\delta}^{2} \boldsymbol{u}:=\boldsymbol{u}_{s s}^{\prime \prime}\left(\underline{s} ; \delta \underline{s}_{1}, \delta \underline{s}_{2}\right)$ of (A-1a) into (A-1b) may be used to evaluate $q^{\prime \prime}\left(\underline{s} ; \delta \underline{s}_{1}, \delta \underline{s}_{2}\right)$. With this result for $q^{\prime \prime}$ we introduce the Hessian of the quantity of interest

$$
\underline{H}=\nabla^{2} q(\underline{s}) \in \mathcal{S} \times \mathcal{S}, \quad \text { where }\left(\nabla^{2} q(\underline{s})\right)_{i j}=q^{\prime \prime}\left(\underline{s} ; \underline{e}_{i}, \underline{e}_{j}\right), i, j=1, \ldots, n_{s} .
$$

Then, for the case of simple constraints in (24), the sufficient condition (27b) simplifies in terms of a reduced Hessian $\underline{\bar{H}}$ as [Bertsekas 1982]

$$
\delta \underline{s}^{T} \underline{\bar{H}} \delta \underline{s} \geq \gamma\|\delta \underline{s}\|^{2} \quad \text { for all } \bar{\delta} \underline{s} \in \mathbb{R}^{n_{s}-n_{B}},
$$

$$
\text { where } \begin{aligned}
\bar{H}_{I J} & =\left\{\nabla^{2} q(\underline{s})\right\}_{I J}=\frac{\partial^{2} q}{\partial s_{I} \partial s_{J}}, \quad I, J \notin B(\underline{s}), \\
B(\underline{s}) & =\left\{i \mid s_{i}=\left(s_{i, \alpha_{k}}^{L}, \frac{\partial q}{\partial s_{i}} \geq 0\right) \vee s_{i}=\left(s_{i, \alpha_{k}}^{R}, \frac{\partial q}{\partial s_{i}} \leq 0\right)\right\},
\end{aligned}
$$

where $B(\underline{s})$ denotes the set of indices of binding constraints at $\underline{s}$ in accordance with the necessary condition (31) and $n_{B}=\operatorname{dim}\{B(\underline{s})\}$ is its dimension.

A.2. The adjoint state differentiation method. Alternatively to the direct differentiation method in Appendix A.1 the second derivative of the quantity of interest $q^{\prime \prime}\left(\underline{s} ; \delta \underline{s}_{1}, \delta \underline{s}_{2}\right)$ is obtained by exploiting the adjoint equation (36b) analogously to the first-order analysis for derivation of the equations (37). Setting $\delta \boldsymbol{u}=\bar{\delta}^{2} \boldsymbol{u}$ in (36b) and using the result (A-1a) with the choice $\delta \boldsymbol{u}=\boldsymbol{\Lambda}$, we obtain

$$
\begin{array}{r}
Q_{u}^{\prime}\left(\underline{s}, \boldsymbol{u} ; \bar{\delta}^{2} \boldsymbol{u}\right)=-G_{\boldsymbol{u}}^{\prime}\left(\underline{s}, \boldsymbol{u} ; \boldsymbol{\Lambda}, \bar{\delta}^{2} \boldsymbol{u}\right) \\
=G_{\underline{s} \underline{\underline{s}}}^{\prime \prime}\left(\underline{s}, \boldsymbol{u} ; \boldsymbol{\Lambda}, \delta \underline{s}_{1}, \delta \underline{s}_{2}\right)+G_{\boldsymbol{u} \underline{\underline{s}}}^{\prime \prime}\left(\underline{s}, \boldsymbol{u} ; \boldsymbol{\Lambda}, \bar{\delta} \boldsymbol{u}_{1}, \delta \underline{s}_{2}\right) \\
\quad+G_{\underline{s} \boldsymbol{u}}^{\prime \prime}\left(\underline{s}, \boldsymbol{u} ; \boldsymbol{\Lambda}, \delta \underline{s}_{1}, \bar{\delta} \boldsymbol{u}_{2}\right)+G_{\boldsymbol{u} \boldsymbol{u}}^{\prime \prime}\left(\underline{s}, \boldsymbol{u} ; \boldsymbol{\Lambda}, \bar{\delta} \boldsymbol{u}_{1}, \bar{\delta} \boldsymbol{u}_{2}\right) \\
\quad \text { for all } \delta \underline{s}_{1}, \delta \underline{s}_{2} \in \mathcal{S}_{\alpha_{k}},
\end{array}
$$

where $\bar{\delta} \boldsymbol{u}_{k}=\boldsymbol{u}_{s}^{\prime}\left(\underline{s} ; \delta \underline{s}_{k}\right), k=1,2$, and where it is noteworthy to recall the relation $\left.\bar{\delta}^{2} \boldsymbol{u}=\boldsymbol{u}_{\underline{s}}^{\prime \prime} \underline{\underline{s}} ; \delta \underline{s}_{1}, \delta \underline{s}_{2}\right)$. Inserting the result (A-4) into (A-1b), the required result for the second derivative of the quantity of interest by the adjoint state method 
becomes

$$
\begin{aligned}
q^{\prime \prime}\left(\underline{s} ; \delta \underline{s}_{1}, \delta \underline{s}_{2}\right)=Q_{\underline{s} \underline{s}}^{\prime \prime} & \left(\underline{s}, \boldsymbol{u} ; \delta \underline{s}_{1}, \delta \underline{s}_{2}\right)+Q_{\underline{u} \underline{\underline{s}}}^{\prime \prime}\left(\underline{s}, \boldsymbol{u} ; \bar{\delta} \boldsymbol{u}_{1}, \delta \underline{s}_{2}\right) \\
& +Q_{\underline{s} \boldsymbol{u}}^{\prime \prime}\left(\underline{s}, \boldsymbol{u} ; \delta \underline{s}_{1}, \bar{\delta} \boldsymbol{u}_{2}\right)+Q_{\boldsymbol{u} \boldsymbol{u}}^{\prime \prime}\left(\underline{s}, \boldsymbol{u} ; \bar{\delta} \boldsymbol{u}_{1}, \bar{\delta} \boldsymbol{u}_{2}\right) \\
& +G_{\underline{s} \underline{s}}^{\prime \prime}\left(\underline{s}, \boldsymbol{u} ; \boldsymbol{\Lambda}, \delta \underline{s}_{1}, \delta \underline{s}_{2}\right)+G_{\boldsymbol{u} \underline{\underline{s}}}^{\prime \prime}\left(\underline{s}, \boldsymbol{u} ; \boldsymbol{\Lambda}, \bar{\delta} \boldsymbol{u}_{1}, \delta \underline{s}_{2}\right) \\
& +G_{\underline{s} \boldsymbol{u}}^{\prime \prime}\left(\underline{s}, \boldsymbol{u} ; \boldsymbol{\Lambda}, \delta \underline{s}_{1}, \bar{\delta} \boldsymbol{u}_{2}\right)+G_{\boldsymbol{u} \boldsymbol{u}}^{\prime \prime}\left(\underline{s}, \boldsymbol{u} ; \boldsymbol{\Lambda}, \bar{\delta} \boldsymbol{u}_{1}, \bar{\delta} \boldsymbol{u}_{2}\right) \\
& \quad \text { for all } \delta \underline{s}_{1}, \delta \underline{s}_{2} \in \mathcal{S}_{\alpha_{k}} ;
\end{aligned}
$$

compare this to [Vexler 2004].

\section{Appendix B: Sensitivity analysis for large strain formulations}

This appendix provides an overall summary for determination of the state problem (6) as a variational formulation, the generally unsymmetric tangent bilinear form (8) of the Newton method for FEM equilibrium iteration, and the gradient $\nabla_{s} f$ for the projection algorithm (47), provided the underlying model equations are formulated within a large strain framework. More details and related references are documented, e.g., in [Barthold 1993; Mahnken 2004; Mahnken and Stein 1997].

B.1. Time and design (tangent) derivatives of kinematic variables. From the fundamental mapping in (3) the design derivative is

$$
\boldsymbol{\varphi}_{\underline{s}}^{\prime}(\boldsymbol{X}, t, \underline{s} ; \delta \underline{s})=\left(\partial_{X} \boldsymbol{\varphi}(\boldsymbol{X}, t, \underline{s}) \cdot \partial_{\underline{s}} \boldsymbol{X}+\partial_{t} \boldsymbol{\varphi}(\boldsymbol{X}, t, \underline{s}) \cdot \partial_{\underline{s}} t+\partial_{\underline{s}}^{p} \boldsymbol{\varphi}(\boldsymbol{X}, t, \underline{s})\right) \cdot \delta \underline{s},
$$

where $\partial_{X} \varphi(X, t, \underline{s}) \in \mathbb{R}^{n_{\mathrm{dim}}} \times \mathbb{R}^{n_{\mathrm{dim}}}$ and $\partial_{t} \boldsymbol{\varphi} \in \mathbb{R}^{n_{\mathrm{dim}}} \times \mathcal{T}$ are partial tangents in the direction of $\boldsymbol{X}$ and $t$, respectively, and the notation

$$
\partial_{\underline{s}}^{p}\{\cdot(\boldsymbol{\varphi}(\boldsymbol{X}, t, \underline{s}), \underline{s})\}:=\partial_{\underline{s}}\{\cdot(\underline{s})\}
$$

is used, thus taking into account explicit dependence of the design variables.

It follows that formulations associated with the time derivative of a kinematic quantity carry over to the design derivative with minor modifications. To give an example, we consider the right Cauchy-Green tensor $\boldsymbol{C}=\boldsymbol{F}^{T} \boldsymbol{F}$ with the deformation gradient in (4a). Then the time and design tangents of $\boldsymbol{C}$ are

$$
\partial_{t} \boldsymbol{C}=2 \operatorname{sym}(\boldsymbol{C L})=: 2 \boldsymbol{D}, \quad \partial_{\underline{s}} \boldsymbol{C}=2 \operatorname{sym}\left(\boldsymbol{C} \boldsymbol{L}_{\underline{s}}\right)=: 2 \boldsymbol{D}_{\underline{s}} .
$$

According to Table B.1, $\boldsymbol{L}$ and $\boldsymbol{L}_{s}$ are material velocity gradients and $\boldsymbol{D}$ and $\boldsymbol{D}_{s}$ are material rate of deformation tensors with respect to the reference configuration. Some further examples of the analogy for time and design derivatives are also listed in Table B.1. Here $\boldsymbol{l}_{t}$ and $\boldsymbol{l}_{\underline{s}}$ are spatial velocity gradients induced by the velocities $\boldsymbol{v}_{t}$ and $\boldsymbol{v}_{\underline{s}}$, respectively, and $\boldsymbol{d}_{t}$ and $\boldsymbol{d}_{\underline{s}}$ are the associated spatial rate of deformation tensors [Barthold 1993; Mahnken 2004]. Also, the symmetric operator $\operatorname{sym}(\cdot):=$ $\frac{1}{2}\left((\cdot)^{T}+(\cdot)\right)$ and the covariant metric tensor $\boldsymbol{g}^{\mathrm{b}}$ are used. $\mathcal{L}_{t}$ denotes the Lie 


\begin{tabular}{|c|c|c|c|}
\hline$\dot{\boldsymbol{F}}=\nabla_{\boldsymbol{X}} \partial_{t} \boldsymbol{\varphi}$ & $\partial_{\underline{S}} \boldsymbol{F}=\nabla_{\boldsymbol{X}} \boldsymbol{\varphi}_{\underline{\underline{S}}}^{\prime}$ & $\partial_{\delta} \boldsymbol{F}=\nabla_{\boldsymbol{X}} \delta \boldsymbol{u}$ & $\partial_{\Delta} \boldsymbol{F}=\nabla_{\boldsymbol{X}} \Delta \boldsymbol{u}$ \\
\hline $\boldsymbol{L}=\boldsymbol{F}^{-1} \dot{\boldsymbol{F}}$ & $\boldsymbol{L}_{\underline{S}}=\boldsymbol{F}^{-1} \partial_{\underline{S}} \boldsymbol{F}$ & $\boldsymbol{L}_{\delta}=\boldsymbol{F}^{-1} \partial_{\delta} \boldsymbol{F}$ & $\boldsymbol{L}_{\Delta}=\boldsymbol{F}^{-1} \partial_{\Delta} \boldsymbol{F}$ \\
\hline $\boldsymbol{D}=\operatorname{sym}(\boldsymbol{C} \boldsymbol{L})$ & $\boldsymbol{D}_{\underline{S}}=\operatorname{sym}\left(\boldsymbol{C} \boldsymbol{L}_{\underline{S}}\right)$ & $\boldsymbol{D}_{\delta}=\operatorname{sym}\left(\boldsymbol{C} \boldsymbol{L}_{\delta}\right)$ & $\boldsymbol{D}_{\Delta}=\operatorname{sym}\left(\boldsymbol{C} \boldsymbol{L}_{\Delta}\right)$ \\
\hline $\boldsymbol{l}=\dot{\boldsymbol{F}} \boldsymbol{F}^{-1}$ & $\boldsymbol{l}_{\underline{S}}=\partial_{\underline{S}} \boldsymbol{F} \boldsymbol{F}^{-1}$ & $\boldsymbol{l}_{\delta}=\partial_{\delta} \boldsymbol{F} \boldsymbol{F}^{-1}$ & $\boldsymbol{l}_{\Delta}=\partial_{\Delta} \boldsymbol{F} \boldsymbol{F}^{-1}$ \\
\hline $\boldsymbol{d}=\operatorname{sym}\left(\boldsymbol{g}^{\mathrm{b}} \boldsymbol{l}\right)$ & $\boldsymbol{d}_{\underline{S}}=\operatorname{sym}\left(\boldsymbol{g}^{\mathrm{b}} \boldsymbol{l}_{\underline{\underline{S}}}\right)$ & $\boldsymbol{d}_{\delta}=\operatorname{sym}\left(\boldsymbol{g}^{\mathrm{b}} \boldsymbol{l}_{\delta}\right)$ & $\boldsymbol{d}_{\Delta}=\operatorname{sym}\left(\boldsymbol{g}^{\mathrm{b}} \boldsymbol{l}_{\Delta}\right)$ \\
\hline $\mathcal{L}_{t}(\cdot)=\Phi_{*}\left[\partial_{t} \Phi^{*}(\cdot)\right]$ & $\mathcal{L}_{\underline{S}}(\cdot)=\Phi_{*}\left[\partial_{\underline{S}} \Phi^{*}(\cdot)\right]$ & $\mathcal{L}_{\delta}(\cdot)=\Phi_{*}\left[\partial_{\delta} \Phi^{*}(\cdot)\right]$ & $\mathcal{L}_{\Delta}(\cdot)=\Phi_{*}\left[\partial_{\Delta} \Phi^{*}(\cdot)\right]$ \\
\hline
\end{tabular}

Table B.1. Examples for kinematic variables induced by time derivatives, design variation, virtual variation, and linearization increment.

(time) derivative of a spatial argument, with related pull-back $\Phi^{*}$ and push-forward operators $\Phi_{*}$.

For the variational formulation in Section 2, two additional types of quantities are useful: the virtual displacements $\delta \boldsymbol{u}$ with $\left.\delta \boldsymbol{u}\right|_{\partial_{\varphi} \mathcal{B}}=\boldsymbol{\Gamma}$ and the increments for linearization $\Delta \boldsymbol{u}$ with $\left.\Delta \boldsymbol{u}\right|_{\partial_{\varphi} \mathcal{B}}=\boldsymbol{\Gamma}$, both independent of $(t, \underline{s})$. With these quantities additional kinematical variables are defined in Table B.1, induced by $\delta \boldsymbol{u}$ or $\Delta \boldsymbol{u}$, respectively, in complete analogy to $\partial_{t} \varphi$ and $\varphi_{s}^{\prime}$, respectively. In Table B.1, ${ }^{*} \Phi_{e}^{\sharp}$ and ${ }_{*} \Phi_{e}^{\sharp}$ are pull-back and push-forward operators of a contravariant tensor according to (B-4) [Mahnken 2005]

$$
{ }^{*} \Phi_{e}^{\sharp}\left[\overline{\boldsymbol{a}}^{\sharp}\right]=\boldsymbol{F}_{\mathrm{el}}^{-1} \cdot \overline{\boldsymbol{a}}^{\sharp} \cdot \boldsymbol{F}_{\mathrm{el}}^{-t}, \quad * \Phi_{e}^{\sharp}\left[\overline{\boldsymbol{A}}^{\sharp}\right]=\boldsymbol{F}_{\mathrm{el}} \cdot \overline{\boldsymbol{A}}^{\sharp} \cdot \boldsymbol{F}_{\mathrm{el}}^{t} \cdot
$$

B.2. Time and design (tangent) derivatives of stress tensors. For alleviation of notation we shall restrict ourselves to large strain elasticity with homogeneous material governed by the local constitutive equations for the second Piola-Kirchhoff stress tensor $\boldsymbol{T}=J \boldsymbol{F}^{-1} \boldsymbol{\sigma} \boldsymbol{F}^{-T}$ dependent on the right Cauchy-Green strain tensor $\boldsymbol{C}$. We remark that the results obtained below also hold for general constitutive models which incorporate inelastic behavior. Then, due to the functional relationship $\boldsymbol{T}=\hat{\boldsymbol{T}}(\hat{\boldsymbol{C}}(\boldsymbol{\varphi}(\boldsymbol{X}, t, \underline{s})), \underline{s})$ the dependence of $\hat{\boldsymbol{T}}$ with respect to time $t$ is implicit, while the dependence on the design variables $\underline{s}$ is both explicit and implicit. Consequently the time and design tangents of $\boldsymbol{T}$ are

$$
\partial_{t} \boldsymbol{T}=\mathbb{C}: \boldsymbol{D}_{t}, \quad \partial_{\underline{s}} \boldsymbol{T}=\mathbb{C}: \boldsymbol{D}_{\underline{s}}+\partial_{\underline{s}}^{p} \boldsymbol{T},
$$

where $\mathbb{C}:=2 \partial_{\boldsymbol{C}} \boldsymbol{T}$ is the symmetric fourth-order material operator, and where the material deformation rate tensors $\boldsymbol{D}_{t}$ and $\boldsymbol{D}_{s}$ are defined in Table B.1. Here the notation (B-2) is used, thus taking into account explicit dependence of the design variables. Comparatively, the time tangent of $\boldsymbol{T}$ consists of one part, while the design tangent consists of two parts. 


\section{Material formulation}

- State equation as weak form

$G=\left\langle\boldsymbol{T}: \boldsymbol{D}_{\delta}\right\rangle-\bar{l}=0$

- Tangent bilinear form for Newton method

$G_{\boldsymbol{u}}^{\prime}=\left\langle\left(\mathbb{C}: \boldsymbol{D}_{\Delta}\right): \boldsymbol{D}_{\delta}+\boldsymbol{L}_{\Delta} \boldsymbol{T}: \boldsymbol{D}_{\delta}\right\rangle$

- Tangent problem for parameter sensitivity $g^{\prime}(\underline{s} ; \delta \underline{s})=\left\langle\left(\mathbb{C}: \boldsymbol{D}_{\underline{s}}\right): \boldsymbol{D}_{\delta}+\boldsymbol{L}_{\underline{s}} \boldsymbol{T}: \boldsymbol{D}_{\delta}+\partial_{\underline{s}}^{p} \boldsymbol{T}: \boldsymbol{D}_{\delta}\right\rangle$ $=0$

$$
\begin{aligned}
& \text { Spatial formulation } \\
& G=\left\langle\boldsymbol{\tau}: \boldsymbol{d}_{\delta}\right\rangle-\bar{l}=0 \\
& G_{\boldsymbol{u}}^{\prime}=\left\langle\left(\mathbb{c}: d_{\Delta}\right): d_{\delta}+l_{\Delta} \tau: d_{\delta}\right\rangle \\
& g^{\prime}(\underline{s} ; \delta \underline{s})=\left\langle\left(\mathbb{C}: \boldsymbol{d}_{\underline{s}}\right): \boldsymbol{d}_{\delta}+\boldsymbol{l}_{\underline{s}} \boldsymbol{\tau}: \boldsymbol{d}_{\delta}+\partial_{\underline{s}}^{p} \boldsymbol{\tau}: \boldsymbol{d}_{\delta}\right\rangle \\
& =0
\end{aligned}
$$

Table B.2. State equation, linearization, and linear equation for parameter sensitivity in a material and a spatial formulation.

For the Kirchhoff stress tensor, which is obtained by push-forward of the second Piola-Kirchhoff stress tensor $\tau=\Phi_{*}(\boldsymbol{T})$, the associated time and design tangents are

$$
\partial_{t} \boldsymbol{\tau}=\mathbb{C}: \boldsymbol{d}_{t}+2 \operatorname{sym}\left(\boldsymbol{l}_{t} \boldsymbol{\tau}\right), \quad \partial_{\underline{s}} \boldsymbol{\tau}=\mathbb{C}: \boldsymbol{d}_{\underline{s}}+2 \operatorname{sym}\left(\boldsymbol{l}_{\underline{s}} \boldsymbol{\tau}\right)+\partial_{\underline{s}}^{p} \boldsymbol{\tau}
$$

and where $\partial_{\underline{s}}^{p} \boldsymbol{\tau}:=\boldsymbol{F} \partial_{\underline{s}}^{p} \boldsymbol{T} \boldsymbol{F}^{T}$. Here the spatial tangent module $\mathbb{C}$ is obtained by pushing forward its material counterpart, i.e., $\mathbb{C}=\Phi_{*}(\mathbb{C})$. Comparing the results for the time and design derivatives of the kinematic variable $C$ in (B-3) with the results of (B-5) and (B-6) for the stress tensors, it is observed that in (B-3) the structure for both derivatives is identical whereas in (B-5) and (B-6) the structures are different.

B.3. Numerical solution of the direct and the inverse problem. With the notations of the previous subsection the direct problem (6) as a weak form of the momentum equation is given in a material setting and a spatial setting in Table B.2. Here the rate of deformation tensor $\boldsymbol{D}_{\delta}$ induced by the virtual displacement $\delta \boldsymbol{u}$ is defined analogously to $\boldsymbol{D}_{t}$ and $\boldsymbol{D}_{\underline{s}}$ in Table B.1, and $\bar{l}$ designates the external part of the weak form.

The iterative solution of the state problem (6) is based on a Newton method (8) with tangent bilinear form $G_{\boldsymbol{u}}^{\prime}=G_{\boldsymbol{u}}^{\prime}(\underline{s}, \tilde{\boldsymbol{u}}(\underline{s}) ; \delta \boldsymbol{u}, \Delta \boldsymbol{u})$ summarized in Table B.2, both in a material and a spatial setting. The rate of deformation tensor $\boldsymbol{D}_{\Delta}$ induced by the incremental configuration $\Delta \varphi$ is defined in complete analogy to $\boldsymbol{D}_{t}$ and $\boldsymbol{D}_{\underline{s}}$ in Table B.1. The iterative solution of the minimization problem (45) is based on the iteration scheme (47). According to (29a) the tangent problem $g^{\prime}(\underline{s} ; \delta \underline{s})=$ $G_{s}^{\prime}(\underline{s}, \boldsymbol{u}(\underline{s}) ; \delta \boldsymbol{u}, \delta \underline{s})+G_{\boldsymbol{u}}^{\prime}(\underline{s}, \boldsymbol{u}(\underline{s}) ; \delta \boldsymbol{u}, \Delta \overline{\boldsymbol{u}})=0$, summarized in Table B.2 both in a material and a spatial setting, has to be solved at each iteration step for calculation of the sensitivity $\Delta \overline{\boldsymbol{u}}=\nabla \boldsymbol{u}(\underline{s}) \cdot \delta \underline{s}, \nabla \boldsymbol{u}(\underline{s}) \in \mathscr{U}^{0} \times \mathcal{S}$. As outlined extensively in [Mahnken and Stein 1997], solution of the tangent problem for history-dependent problems requires an additional postprocessing step for the history-dependent sensitivities. 


\section{References}

[Akpan et al. 2001] U. O. Akpan, T. S. Koko, I. R. Orisamolu, and B. K. Gallant, "Practical fuzzy finite element analysis of structures", Finite Elem. Anal. Des. 38:2 (2001), 93-111.

[Andresen et al. 1996] K. Andresen, S. Dannemeyer, H. Friebe, R. Mahnken, R. Ritter, and E. Stein, "Parameteridentifikation für ein plastisches Stoffgesetz mit FE-Methoden und Rasterverfahren", Bauingenieur 71 (1996), 21-31.

[Bangerth and Rannacher 2003] W. Bangerth and R. Rannacher, Adaptive finite element methods for differential equations, Birkhäuser, 2003.

[Banks and Kunisch 1989] H. T. Banks and K. Kunisch, Estimation techniques for distributed parameter systems, Systems \& Control: Foundations \& Applications 1, Birkhäuser, 1989.

[Barthold 1993] F.-J. Barthold, Theorie und Numerik zur Berechnung und Optimierung von Strukturen aus isotropen, hyperelastischen Materialien, Dr.-Ing. Dissertation, Universität Hannover, 1993.

[Bertsekas 1982] D. P. Bertsekas, "Projected Newton methods for optimization problems with simple constraints", SIAM J. Control Optim. 20:2 (1982), 221-246.

[Caylak and Mahnken 2012] I. Caylak and R. Mahnken, "Stabilization of mixed tetrahedral elements at large deformations", Internat. J. Numer. Meth. Eng. 90:2 (2012), 218-242.

[De Munck et al. 2008] M. De Munck, D. Moens, W. Desmet, and D. Vandepitte, “A response surface based optimisation algorithm for the calculation of fuzzy envelope FRFs of models with uncertain properties", Comput. Struct. 86:10 (2008), 1080-1092.

[Dennis and Schnabel 1983] J. E. Dennis, Jr. and R. B. Schnabel, Numerical methods for unconstrained optimization and nonlinear equations, Prentice Hall, 1983.

[Dubois and Prade 1980] D. Dubois and H. Prade, Fuzzy sets and systems: theory and applications, Mathematics in Science and Engineering 144, Academic, 1980.

[Farkas et al. 2008] L. Farkas, D. Moens, D. Vandepitte, and W. Desmet, "Application of fuzzy numerical techniques for product performance analysis in the conceptual and preliminary design stage”, Comput. Struct. 86:10 (2008), 1061-1079.

[Graf et al. 2015] W. Graf, M. Götz, and M. Kaliske, "Analysis of dynamical processes under consideration of polymorphic uncertainty”, Struct. Saf. 52:B (2015), 194-201.

[Johansson et al. 2007] H. Johansson, K. Runesson, and F. Larsson, "Parameter identification with sensitivity assessment and error computation”, GAMM-Mitt. 30:2 (2007), 430-457.

[Johansson et al. 2008] H. k. Johansson, F. Larsson, and K. Runesson, "Estimation of model errors in the calibration of viscoelastic material models", Internat. J. Numer. Meth. Eng. 76:10 (2008), $1568-1582$.

[Luenberger 1984] D. G. Luenberger, Linear and nonlinear programming, 2nd ed., Addison-Wesley, 1984.

[Mahnken 1993] R. Mahnken, Duale Verfahren für nichtlineare Optimierungsprobleme in der Strukturmechanik, Dr.-Ing. Dissertation, Universität Hannover, 1993.

[Mahnken 2004] R. Mahnken, "Identification of material parameters for constitutive equations", Chapter 19, pp. 637-655 in Encyclopedia of computational mechanics, vol. 2: Solids and structures, edited by E. Stein et al., Wiley, 2004.

[Mahnken 2005] R. Mahnken, "Void growth in finite deformation elasto-plasticity due to hydrostatic stress states", Comput. Meth. Appl. Mech. Eng. 194:34-35 (2005), 3689-3709.

[Mahnken 2013] R. Mahnken, "Goal-oriented adaptive refinement for phase field modeling with finite elements”, Internat. J. Numer. Meth. Eng. 94:4 (2013), 418-440. 
[Mahnken and Stein 1996] R. Mahnken and E. Stein, "Parameter identification for viscoplastic models based on analytical derivatives of a least-squares functional and stability investigations", Int. J. Plasticity 12:4 (1996), 451-479.

[Mahnken and Stein 1997] R. Mahnken and E. Stein, "Parameter identification for finite deformation elasto-plasticity in principal directions", Comput. Meth. Appl. Mech. Eng. 147:1-2 (1997), 17-39.

[Marsden and Hughes 1994] J. E. Marsden and T. J. R. Hughes, Mathematical foundations of elasticity, Dover, 1994.

[Möller and Beer 2004] B. Möller and M. Beer, Fuzzy randomness: uncertainty in civil engineering and computational mechanics, Springer, 2004.

[Möller et al. 2000] B. Möller, W. Graf, and M. Beer, "Fuzzy structural analysis using $\alpha$-level optimization", Comput. Mech. 26:6 (2000), 547-565.

[Muhanna and Mullen 1999] R. L. Muhanna and R. L. Mullen, "Formulation of fuzzy finite-element methods for solid mechanics problems", Comput.-Aided Civ. Inf. 14:2 (1999), 107-117.

[Nocedal and Wright 1999] J. Nocedal and S. J. Wright, Numerical optimization, Springer, 1999.

[Oden et al. 2006] J. T. Oden, S. Prudhomme, A. Romkes, and P. T. Bauman, "Multiscale modeling of physical phenomena: adaptive control of models", SIAM J. Sci. Comput. 28:6 (2006), 23592389.

[Ogden 1997] R. W. Ogden, Non-linear elastic deformations, Dover, 1997.

[Press et al. 1992] W. H. Press, S. A. Teukolsky, W. T. Vetterling, and B. P. Flannery, Numerical recipes in FORTRAN: the art of scientific computing, 2nd ed., Cambridge University, 1992.

[Quagliarella and Vicini 1998] D. Quagliarella and A. Vicini, "Coupling genetic algorithms and gradient based optimization techniques", Chapter 14, pp. 298-309 in Genetic algorithms and evolution strategy in engineering and computer science: recent advances and industrial applications, edited by D. Quagliarella et al., Wiley, 1998.

[Rüter 2004] M. Rüter, Error-controlled adaptive finite element methods in large strain hyperelasticity and fracture mechanics, Dr.-Ing. Dissertation, Universität Hannover, 2004.

[Schwefel 1977] H. P. Schwefel, Numerische Optimierung von Computer-Modellen mittels der Evolutionsstrategie: mit einer vergleichenden Einführung in die Hill-Climbing- und Zufallsstrategie, Interdisziplinäre Systemforschung 26, Birkhäuser, 1977.

[Simo and Armero 1992] J. C. Simo and F. Armero, "Geometrically nonlinear enhanced strain mixed methods and the method of incompatible modes", Internat. J. Numer. Meth. Eng. 33:7 (1992), 14131449 .

[Simo and Miehe 1992] J. C. Simo and C. Miehe, "Associative coupled thermoplasticity at finite strains: formulation, numerical analysis and implementation”, Comput. Meth. Appl. Mech. Eng. 98:1 (1992), 41-104.

[Simo and Taylor 1991] J. C. Simo and R. L. Taylor, "Quasi-incompressible finite elasticity in principal stretches: continuum basis and numerical algorithms", Comput. Meth. Appl. Mech. Eng. 85:3 (1991), 273-310.

[Sullivan 2015] T. J. Sullivan, Introduction to uncertainty quantification, Texts in Applied Mathematics 63, Springer, 2015.

[Vexler 2004] B. Vexler, Adaptive finite element methods for parameter identification problems, Dr. Dissertation, Universität Heidelberg, 2004.

[Viertl 1996] R. Viertl, Statistical methods for non-precise data, CRC, 1996.

[Wasfy and Noor 1998] T. Wasfy and A. Noor, "Application of fuzzy sets to transient analysis of space structures", pp. 1172-1182 in 39th AIAA/ASME/ASCE/AHS/ASC Structures, Structural Dynamics, and Materials Conference and Exhibit (Long Beach, CA, 1998), AIAA, 1998. 
[Widany and Mahnken 2012] K.-U. Widany and R. Mahnken, "Adaptivity for parameter identification of incompressible hyperelastic materials using stabilized tetrahedral elements", Comput. Meth. Appl. Mech. Eng. 245-246 (2012), 117-131.

[Zadeh 1965] L. A. Zadeh, "Fuzzy sets", Inform. Control 8 (1965), 338-353.

Received 17 Dec 2016. Accepted 27 Jun 2017.

ROLF MAHNKEN: rolf.mahnken@ltm.upb.de

Paderborn, Germany 


\title{
ON THE NOTION OF STRESS IN CLASSICAL CONTINUUM MECHANICS
}

\author{
Simon R. Eugster AND CHRISTOPH GLOCKER
}

\begin{abstract}
A variational formulation of continuum mechanics, in which the principle of virtual work and the variational law of interaction are postulated as the basic axioms, is still controversially discussed. In particular, not widely accepted is the internal virtual work contribution of a continuum, as postulated as a smooth density integrated over the deformed configuration of the body, in which the stress field is defined as the quantity dual to the gradient of the virtual displacement field. The question arises whether this internal virtual work can be deduced, rather than just postulated, from already known mechanical concepts completely within the variational framework. To achieve such a derivation, we give in this paper an interpretation of Piola's micro-macro identification procedure in view of the Riemann integral, which naturally provides in its mathematical definition a micro-macro relation between the discrete system of infinitesimal volume elements and the continuum. Accordingly, we propose a definition of stress on the micro level of the infinitesimal volume elements. In particular, the stress is defined as the internal force effects of the body that model the mutual force interaction between neighboring infinitesimal volume elements. The internal virtual work of the continuum is then obtained by Piola's micro-macro identification procedure, where in the limit of vanishing volume elements the virtual work of the continuous macromodel is identified with the virtual work of the discrete micromodel. In the course of this procedure, the stress tensor emerges directly as the quantity dual to the gradient of the virtual displacement field. Furthermore, we try to gather important results of variational continuum mechanics, which have appeared here and there in very diverse forms, in order to underline once more the strength of a variational formulation of continuum mechanics.
\end{abstract}

\section{Introduction}

To date, there are essentially two different ways to postulate the foundations of continuum mechanics. The first method, henceforth called the nonvariational approach, was conceived mainly by Cauchy $[1823 ; 1827 \mathrm{a}]$ and assumes forces and

\section{Communicated by Francesco dell'Isola.}

MSC2010: 70A05, 74A10.

Keywords: continuum mechanics, virtual work, law of interaction, stress, micro-macro identification. 
moments as the elemental quantities. The second method, which traces back to Lagrange [1788] and Piola [1832; 1848; 2014; dell' Isola et al. 2015a], is of a variational nature and defines forces in a generalized sense as the quantities dual to the virtual displacement field and the gradients thereof. The variational approach has gained much attention during the last half century, especially in the field of generalized continua like gradient materials and polar and micromorphic media; see for instance [dell'Isola et al. 2015b; 2011; Toupin 1964; Germain 1973b]. Most of these generalized theories cannot be stated straightforwardly by using the nonvariational approach. For an evaluative comparison of both the variational and the nonvariational formulations of continuum mechanics and for further references on this topic, we refer to [dell'Isola et al. 2017] and works cited therein. In what follows and without claiming to be in any way complete, we try to review the vast amount of literature on the various formulations of classical continuum mechanics that has appeared in the last two centuries. Since we focus in this treatise on the notion of stress, we omit the discussion about inertia forces. As a consequence, we refer exclusively to the terminology used in statics, e.g., the equilibrium of forces and moments, instead of addressing the balance of linear and angular momentum.

The first formulation of continuum mechanics can be attributed to Cauchy with his celebrated publications [Cauchy $1823 ; 1827 \mathrm{a}$ ]. Cauchy restricted forces to be of volume and surface nature only. He assumed the force interaction between an arbitrary subbody and the rest of the body to take place exclusively by surface forces, called stress vectors. This requirement is nowadays referred to as the stress principle of Euler and Cauchy [Truesdell and Toupin 1960, §200]. Cauchy implicitly made the assumption, known as Cauchy's postulate, that a stress vector for a given body point depends only on the normal vector to an imagined cutting or contact surface passing through this point. By postulating the equilibrium of forces at an infinitesimal parallelepiped, he proved that the stress vectors acting upon opposite sides of the same surface at a given point are equal in magnitude and opposite in direction, which is referred to as Cauchy's lemma. Applying then the equilibrium of forces at an infinitesimal tetrahedron, Cauchy's stress theorem shows that the stress vector for a body point depends linearly on the normal vector of the imagined contact surface. Consequently, Cauchy's stress theorem asserts the existence of the stress tensor field [Truesdell 1991, pp. 174-175]. Furthermore, by postulating the equilibrium of moments at an infinitesimal parallelepiped, Cauchy proved the symmetry of the stress tensor. ${ }^{1}$ Note that in all proofs boundedness assumptions on the applied external forces are involved. In a further publication,

\footnotetext{
${ }^{1}$ Cauchy's lemma and the symmetry of the stress tensor are formulated in [Cauchy 1827a] as "Théorème I" and "Théorème II", respectively. The celebrated stress theorem of Cauchy has to be extracted out of the text and the formulas on [Cauchy 1827a, pp. 68-69].
} 
Cauchy [1827b] derived from the equilibrium of forces at an infinitesimal parallelepiped together with the results of the stress theorem the local equilibrium equations. Poisson [1829, §11] as well as Thomson and Tait [1867, §698] showed by integrating the local equilibrium equations over a finite volume and by applying the divergence theorem that these equations are the sufficient as well as necessary conditions for the equilibrium of the body. This is, the equilibrium of forces and moments in integral form has to hold for the body and all its subbodies. Lamé $[1852, \S 32]$ and Clebsch $[1862, \S 16]$ derived the (virtual) work contribution of the body. They attained the (virtual) work of the body by multiplying the local equilibrium equations with infinitesimal (virtual) displacements, followed by an integration over the body and application of the divergence theorem, and final usage of the surface boundary conditions.

Kirchhoff [1876, Vorlesung 11] reversed the direction of argumentation and based continuum mechanics on the equilibrium of forces and moments in integral form [Müller and Timpe 1914, p. 23]. Kirchhoff proposed, since every subbody is again a body, that the integral balance laws not only hold for the body, but also for every subbody. By using the results of Cauchy's stress theorem in the integral balance laws and by applying the divergence theorem, the local equilibrium equations as well as the symmetry of the stress tensor can be extracted by a localization argument. Also the axiomatic scheme of Noll [1959] followed the approach of Kirchhoff and highly influenced the celebrated work of Truesdell and Toupin [1960, §196]. As a consequence of the popularity of [Truesdell and Toupin 1960], this approach has become standard in continuum mechanics and can be found in a wealth of modern textbooks [Altenbach and Altenbach 1994; Başar and Weichert 2000; Becker and Bürger 1975; Bertram 2012; Chadwick 1999; Ciarlet 1988; Dvorkin and Goldschmit 2006; Eringen 1980; Gurtin 1981; Haupt 2002; Holzapfel 2000; Liu 2002; Malvern 1965; Ogden 1997; Sedov 1972; Spencer 2004; Truesdell and Noll 1965]. Furthermore, Noll [1974] showed that the balance laws can be derived from the objectivity of the work done by the applied (external) forces and postulated this objectivity as a fundamental axiom of continuum mechanics; see also [Truesdell 1991].

An axiomatization of continuum mechanics at an earlier date and the attempt to partially solve the sixth problem of Hilbert ${ }^{2}$ has been given by Hamel in [1912, $\S 38-39$ ] or [1927, §II.b. $\alpha$ ], whose approach differs from Cauchy's in only one point: instead of claiming the equilibrium of moments at an infinitesimal volume

\footnotetext{
${ }^{2}$ Hilbert's sixth problem was proposed in a lecture at the international congress of mathematicians at Paris in 1900 [1901; 1902] "to treat in the same manner, by means of axioms, those physical sciences in which mathematics plays an important part; in the first rank are the theory of probabilities and mechanics."
} 
element, Hamel demanded the stress tensor be symmetric, which he denoted the Boltzmann axiom.

Cauchy's theory of continuum mechanics is based on the insights of Newton and Euler, where forces and moments are the primitives of mechanics. In contrast, Piola was highly influenced by Lagrange's Mécanique Analytique and the calculus of variation (see the Introduction of [Piola 1832] ${ }^{3}$ ). He understood forces in the sense of duality as linear functionals on virtual displacements. While the theories developed in [Piola 1832] are for rigid bodies only, Piola [1848] formulated his variational theory of mechanics also for deformable bodies, with the principle of virtual work as the fundamental equation. Piola introduced between the reference and the deformed configurations an intermediate configuration which differs from the deformed configuration by only a rigid body motion. The stresses are defined as indeterminate Lagrange multipliers to the rigidity constraints between the intermediate and deformed configuration [Piola 2014, Chapter 1, pp. 83-84]. By claiming the principle of virtual work with its corresponding contributions, Piola obtained by an appropriate substitution of variables and integration by parts the local equilibrium equations of Cauchy and the corresponding force boundary conditions. In fact, Piola introduced the (Cauchy) stress tensor as a linear functional on the symmetrized gradient of the virtual displacement field. As a direct consequence of this definition, which already contains objectivity assumptions, the stress tensor is symmetric. Furthermore, Piola derived the integral balance laws for an arbitrary subbody by choosing virtual displacement fields which respect the rigidity constraint between the intermediate and the deformed configurations [Piola 2014, Chapter 1, pp. 85-86]. A very similar approach, without reference to Piola, can be found in [Hamel 1967, Chapter III, §6]. The variational theory of Piola has been made even clearer by the encyclopedia article [Hellinger 1914]. Hellinger in his theory defined forces and stresses as linear forms on the virtual displacement field and the gradient thereof, and proposed the principle of virtual work as the fundamental equation in continuum mechanics. The gradient of the virtual displacement field does not imply a symmetric stress tensor. Hellinger [1914, p. 619] mentioned therefore that the symmetry follows either from the Boltzmann axiom of Hamel or from the equilibrium of moments in integral form. An exegetic series about [Hellinger 1914] including the complete translation into English has just been finished by Eugster and dell'Isola [2017a; 2017b; 2017c].

After the variational formulation of continuum mechanics was almost buried into oblivion, there has been a renaissance of the variational theory in the 1970s by the publications of Germain [1972; 1973a; 1973b]; see also [Maugin 1980]. Therein, first and second gradient theories as well as theories for continua with

\footnotetext{
${ }^{3}$ An English translation is given in [Capecchi and Ruta 2015, pp. 90-93].

${ }^{4}$ An English translation is given in [Piola 2014].
} 
microstructure have been formulated by defining virtual work contributions in the sense of duality and by the postulation of the principle of virtual work as the basic axiom. Maybe the most important contribution, which varies from Piola and Hellinger, is the explicit statement of the axiom of power of internal forces. This axiom demands the virtual work of internal forces to vanish for all rigid virtual displacements and induces the symmetry of the stress in classical continuum mechanics. To date, textbooks on continuum mechanics based on the variational approach can exclusively be found in France, i.e., [Germain 1986; Lemaitre and Chaboche 1990; Salençon 2000; 2001]. Following the ideas of Germain [1972], dell'Isola et al. [2015b; 2011; 2012] obtain from the theory of distributions by L. Schwartz [1951] a representation of the virtual work in terms of $N$-th order stresses which are defined as the quantities dual to the $N$-th gradients of the virtual displacement field. A similar representation of forces in a continuum has already been proposed by Segev [1986]. In this generalized theory, the classical continuum is embedded and obtained for $N=1$. Consequently, the theory of the classical continuum is also referred to as first gradient theory. Another axiomatization of variational nature, also for an $N$-th gradient continuum, is from Bertram [1989] and Bertram and Forest [2007]. They base continuum mechanics on invariance requirements upon a general principle of virtual power, as a linear and continuous extension of the balance of work. In contrast to [Germain 1972], the theory is formulated for the total virtual power, and there is no a priori partition into internal and external forces.

Poisson [1829, p. 400], before Piola had published his variational theories, criticized the methods of Lagrange for not being suitable for continuum mechanics. Piola was thereby stimulated to defend the variational approach to continuum mechanics; see the introduction of [Piola 1848] $]^{5}$. More than a hundred years later, Truesdell came up with a similar criticism in [Truesdell and Toupin 1960, §231] and charged the theory of Hellinger [1914, §3a] to fail through petitio principii, because the stress components would unmotivatedly appear in the constituting variational principle. To avoid this a priori notion of internal virtual work, Del Piero [2009] proposed an approach based on the objectivity criterion of Noll [1974]. Instead of the work, the virtual work of the external forces is required to be objective in his contribution. Hence, the balance laws can be deduced and used to prove Cauchy's stress theorem. Using in the virtual work of the external forces of an arbitrary subbody the relation between the surface force and the normal vector of the cutting surface, an expression of the internal virtual work is obtained. Knowing the form of the internal virtual work, the principle of virtual work can then be formulated a posteriori as being the balance between the virtual work of external and internal

${ }^{5}$ An English translation is given in [Piola 2014, Chapter 1, p. 4]. 
forces. Continuum mechanics based on requiring objectivity of the external virtual work (or power) can also be found in the textbook of Mariano and Galano [2015]. A similar derivation of the internal virtual work contribution is given in the earlier work of Murnaghan [1937].

In the nonvariational approach the stress principle of Euler and Cauchy defines the notion of stress in the sense that it restricts the interaction between subbodies to take place by contact surface forces only. Hence, the contact interaction between the subbodies is chosen as a basic concept. Together with the equilibrium of forces, the existence of the stress tensor follows from Cauchy's theorem. In contrast, in the variational theory as proposed by Germain [1972; 1973a; 1973b], the stress tensor field is defined as the quantity dual to the gradient of the virtual displacement field. By the application of the divergence theorem, the interaction mechanism between neighboring subbodies, i.e., the contact interaction, follows as a consequence. The latter is in the variational theory a derived concept but not an independent one. As mentioned above, the variational formulation is often criticized due to its a priori definition of the internal virtual work. Consequently, the question arises whether the internal virtual work contribution can be deduced from already known mechanical concepts, but still completely within the variational framework. This is meant to show the existence of the stress tensor, but without using the stress principle of Euler and Cauchy. It seems to the authors that such a derivation has already been obtained in [Piola 1848, Capitolo VI], ${ }^{6}$ "On the motion of a generic deformable body following the ideas of the modern scientists about the molecular actions", where Piola presented a nonlocal continuum theory deduced by the identification of the virtual work contributions of a discrete micromodel with the corresponding virtual work of a continuous macromodel, i.e., the continuum. As proposed by [dell'Isola et al. 2015a], we call this approach Piola's micro-macro identification procedure. Piola [1848, Capitolo II] $]^{7}$ used this micro-macro identification also for external forces when discussing line, surface, and volume forces. Having the discrete molecular and atomistic structure of a body in mind, Piola associated the placements of the material particles of a discrete system with the continuous placement field of the continuum evaluated at distinct points. With a clever scaling of the appearing force quantities, and in the limit of vanishing distances between the infinitely many material points and their neighbors, the sum of all virtual work contributions of the discrete material particles can be rewritten as an integral expression. It is this very integral expression which is identified with the virtual work contribution of the continuous macromodel. In his nonlocal continuum theory, Piola proposes a model in which each material point can interact with all the

\footnotetext{
${ }^{6}$ An English translation is given in [Piola 2014, Chapter 1, pp. 146-164] and commented on in [dell'Isola et al. 2015a].

${ }^{7}$ An English translation is given in [Piola 2014, Chapter 1, pp. 31-75].
} 
other material points of the body. The micro-macro identification procedure then leads straightforwardly to a virtual work contribution having the form of a sixfold integral over the body; see (10) in [dell'Isola et al. 2015a, p. 5]. A subsequent Taylor expansion of the variation of the quadratic distances between the material points, together with painful rearrangements of the force quantities, yields then the virtual work expressions of an $N$-th-gradient theory [dell'Isola et al. 2015a].

In this paper we aim to show that Piola's micro-macro identification procedure, which has fallen into oblivion until its translation into English by dell'Isola et al. [Piola 2014], is a suitable approach to derive the virtual work contributions for a continuum. More precisely, we want to address a derivation in which the internal virtual work contribution of the classical continuum is obtained directly from a micro-macro identification, which does not require the full Taylor expansion of Piola's nonlocal theory. Furthermore, we slightly modify Piola's line of arguments. Instead of motivating the appearing micromodel from the molecular structure of the continuum, we claim that such a micromodel is naturally included in the definition of an integral. We start from the concept of the volume integral appearing in the virtual work as being obtained by a dissection of the body into volume elements followed by a limit process with a refinement of these elements. In this way, the mathematical definition of the volume integral naturally provides a micro-macro relation between the infinitesimal volume elements and the continuum. The identification of the virtual work of the continuous macromodel with the virtual work of the discrete micromodel in the limit of vanishing volume elements can then be understood as Piola's micro-macro identification procedure. Within this context, we propose in addition a definition of stress as the internal force effects of the body on the micro level, which model the interaction between neighboring infinitesimal volume elements. The very same perception of stress has already been formulated by Boltzmann in his Populäre Schriften:

"What now concerns the forces which the volume elements of solid bodies mutually exert on each other, one must assume, that each volume element acts on only its direct neighbors, and that it exerts forces on all points adjacent to the cutting surface, which act just as if pulling threads under tension or pushing supporting bars were attached to it." 8

Within the limit process of vanishing volume elements, the summation of all virtual work contributions of the force interactions between the volume elements of the body leads to the appearance of the stress tensor in the internal virtual work contribution of the continuum. Hence, using the definition of the stress on the micro level of the infinitesimal volume elements in the sense of Boltzmann together with Piola's micro-macro identification procedure, the existence of the stress tensor and

${ }^{8}$ This is an English translation of [Boltzmann 1905, p. 297]. 
consequently the internal virtual work of the continuum can be derived without using the stress principle of Euler and Cauchy. The variational law of interaction, which requires the internal virtual work to vanish for all rigid virtual displacements, implies in a further step the symmetry of the stress tensor. With this alternative perspective on the concept of stress, it is possible to shed some light on the internal virtual work of the continuum and to formulate mechanics completely within a variational framework, in which the principle of virtual work and the variational law of interaction emerge as fundamental axioms of mechanics.

The outline of the paper is as follows. In Section 2, the required kinematics of the continuum is introduced. Section 3 proposes a variational theory of mechanics, mainly based on the insights of [Germain 1972; dell'Isola et al. 2012], with the principle of virtual work and the variational law of interaction as the basic axioms. The core of this paper is formulated in Section 4: based on an alternative definition of stress on the micro level of infinitesimal volume elements and Piola's micro-macro identification procedure, the virtual work contribution of the stress is derived on the macro level, together with the emerging stress tensor. In Section 5 the symmetry of the stress tensor is shown as a consequence of the variational law of interaction. The invariance of the virtual work with respect to different integral parametrizations and coordinate representations induces transformation properties of the appearing force quantities. A small selection of transformation properties for the stress is shown in Section 6. Section 7 derives the boundary value problem from the principle of virtual work for the classical assumption that external forces contribute either as volume forces in the interior of the body or as surface forces on the boundary. For the very same assumptions on the external forces, the integral balance laws are derived in Section 8. In the course of this, the inverse stress theorem appears which answers the question how a subbody interacts with its complement. Finally, conclusions are drawn in Section 9.

\section{Kinematics of the continuum}

In this section we introduce briefly the kinematical objects required for the variational formulation of continuum mechanics. The physical space is represented by the three-dimensional Euclidean vector space $\mathbb{E}^{3}$, equipped with an orthonormal basis $\left(\boldsymbol{e}_{x}^{I}, \boldsymbol{e}_{y}^{I}, \boldsymbol{e}_{z}^{I}\right)$ and an origin $O$, subsequently called the $I$-system. A point of the physical space is addressed by the position vector $x \in \mathbb{E}^{3}$. The cartesian coordinate representation of the position vector $\boldsymbol{x}$ in the $I$-system is denoted as

$$
{ }_{I} \boldsymbol{x}:=\left(\begin{array}{l}
x \\
y \\
z
\end{array}\right) \quad \text { with } \boldsymbol{x}=x \boldsymbol{e}_{x}^{I}+y \boldsymbol{e}_{y}^{I}+z \boldsymbol{e}_{z}^{I}
$$




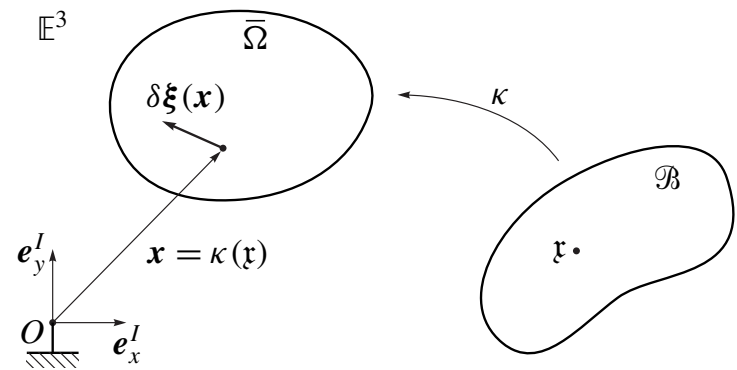

Figure 1. The placement $\kappa$ of the body $\mathscr{B}$ into the Euclidean vector space $\mathbb{E}^{3}$. At every material point $\boldsymbol{x}$ in the deformed configuration $\bar{\Omega}$, a virtual displacement $\delta \boldsymbol{\xi}(\boldsymbol{x})$ can be evaluated.

The body $\mathscr{\Re}$, as suggested by [Noll 1959; Segev 1986], is a three-dimensional compact differentiable manifold. A point of the body manifold $\mathfrak{x} \in \mathscr{B}$ is called a material point of $\mathscr{B}$. The placement of $\mathscr{B}$, as depicted in Figure 1 , is the embedding of the body into the physical space, i.e., the mapping $\kappa: \mathscr{B} \rightarrow \mathbb{E}^{3}$. Embeddings as proper injective immersions respect the principle of impenetrability and the permanence of matter; see [Truesdell and Toupin 1960, §16] for their specific definitions. The image $\bar{\Omega}=\kappa(\mathscr{B}) \subset \mathbb{E}^{3}$ is the region in the physical space occupied by the body manifold and is called the deformed configuration. The deformed configuration as a closed subset ${ }^{9}$ can be written as a disjoint union $\bar{\Omega}=\Omega \cup \partial \Omega$ of the interior $\Omega$ and the boundary $\partial \Omega$ of the deformed configuration, respectively. A spatial point $\boldsymbol{x} \in \bar{\Omega}$ is called a material point in the deformed configuration $\bar{\Omega}$.

A variational family of placements is a differentiable parametrization of placements $\bar{\kappa}(\varepsilon, \mathfrak{x})$ with respect to a single parameter $\varepsilon \in \mathbb{R}$, such that $\kappa(\mathfrak{x})=\bar{\kappa}\left(\varepsilon_{0}, \mathfrak{x}\right)$. The virtual displacement field $\delta \xi: \bar{\Omega} \rightarrow \mathbb{E}^{3}$ in the deformed configuration is the smooth vector field over $\bar{\Omega}$ defined as

$$
\delta \boldsymbol{\xi}(\boldsymbol{x}):=\frac{\partial \bar{\kappa}}{\partial \varepsilon}\left(\varepsilon_{0}, \kappa^{-1}(\boldsymbol{x})\right),
$$

which corresponds to the variation of the placement $\kappa$ evaluated at the material point $\mathfrak{x}=\kappa^{-1}(\boldsymbol{x})$. When no body manifold is available or not of interest as for instance in fluid mechanics where often only control volumes in space are considered, the virtual displacement field can be directly defined as a smooth vector field over the deformed configuration $\bar{\Omega}$. By multiplying (2) with a small $\delta \varepsilon=\left(\varepsilon-\varepsilon_{0}\right)$, the resulting fields can be interpreted as infinitesimal displacements of the deformed

\footnotetext{
${ }^{9} \mathrm{~A}$ compact manifold embedded into the Euclidean space $\mathbb{E}^{3}$ is a closed subset of $\mathbb{E}^{3}$, as is evident by the following two propositions from set theory [Munkres 2000]. Proposition 1: The image of a compact set under a continuous map is compact. Proposition 2: A compact set of a Hausdorff space is closed. Since an embedding is a continuous map and the Euclidean vector space is a Hausdorff space, the assertion follows immediately.
} 
configuration. A subset of such displacements are displacements which shift the deformed configuration infinitesimally as if it was the configuration of a rigid body. The corresponding virtual displacements constitute the subset of rigid virtual displacements $\delta \xi_{\text {rig }}$ which can be parametrized for instance as

$$
\delta \boldsymbol{\xi}_{\text {rig }}(\boldsymbol{x})=\delta \boldsymbol{r}_{O}+\delta \boldsymbol{\phi} \times \boldsymbol{x},
$$

where $\delta \boldsymbol{r}_{O} \in \mathbb{E}^{3}$ and $\delta \boldsymbol{\phi} \in \mathbb{E}^{3}$ denote the virtual displacement of the origin $O$ and the virtual rotation, respectively. The virtual rotation vector $\delta \phi$ or, more precisely, the virtual rotation operator $\delta \phi \times$ will mainly be used throughout the text instead of the skew-symmetric linear map $\tilde{\boldsymbol{\phi}}(\varepsilon)$ with $\tilde{\boldsymbol{\phi}}\left(\varepsilon_{0}\right)=0$, which provides via $\overline{\boldsymbol{\xi}}(\varepsilon, \boldsymbol{x})=$ $\exp (\tilde{\boldsymbol{\phi}}(\varepsilon)) \boldsymbol{x}$ a variational family of rigid rotations.

\section{The axioms of mechanics}

Once we have introduced the kinematics of the continuum, we briefly reconsider the axiomatic scheme of variational continuum mechanics proposed by Germain [1972; 1973b]. Following Lagrange's ideas, we start with the mechanical definition of forces and consider them as quantities dual to the virtual displacements. As a consequence and as stated for instance in [dell'Isola et al. 2015b; 2011; 2012], we therefore accept the idea of considering forces as distributions in the sense of L. Schwartz [1951]. This definition already uses the concept of duality and motivates the formulation of the equilibrium conditions in variational form, which is the first axiom, the principle of virtual work. In an upcoming step, internal and external forces are defined in order to impose a certain variational condition on the internal forces. This is the second axiom, the variational law of interaction.

Definition 1 (mechanical definition of force). We define force as a linear functional $\boldsymbol{F}$ on the space of virtual displacement fields $\delta \boldsymbol{\xi}$, which associates with every $\delta \boldsymbol{\xi}$ a real number $\delta W=\boldsymbol{F}(\delta \xi)$ called virtual work.

For a continuum, the space of virtual displacement fields is given by all smooth vector fields over the body $\bar{\Omega}$. Choosing appropriate compact supports for these vector fields, and following the results of the theory of distributions, forces can be represented by the map

$$
\delta \boldsymbol{\xi} \mapsto \delta W=\boldsymbol{F}(\delta \boldsymbol{\xi}) \quad \text { with } \boldsymbol{F}(\delta \boldsymbol{\xi})=\sum_{k=0}^{N} \int_{\bar{\Omega}} \frac{\partial^{k}}{\partial \boldsymbol{x}^{k}}(\delta \boldsymbol{\xi}) \mid \mathrm{d} \boldsymbol{F}^{k},
$$

where $\partial^{k} / \partial \boldsymbol{x}^{k}$ denotes the $k$-th partial derivative with respect to $\boldsymbol{x}$, the force measure $\mathrm{d} \boldsymbol{F}^{k}$ is a tensor-valued measure of rank $k+1$ having support in $\bar{\Omega}$, and the symbol $\mid$ stands for the complete contraction of the tensors, i.e., a $(k+1)$ contraction. We refer to [dell'Isola et al. 2015b] for more technical details about 
this result. Remark that (4) gives a much larger spectrum of force representations than required for the classical continuum theory. However, it is precisely one of the aims of this paper to deduce, by an appropriate micro-macro identification, the force representations for the interactions of the classical theory.

Contained in the integral of (4) is that the $k$-th derivative of the virtual displacement and the corresponding force measure $\mathrm{d} \boldsymbol{F}^{k}$ have to be evaluated at the very same point $\boldsymbol{x} \in \bar{\Omega}$ and are then to be contracted, which constitutes the duality pairing and which may be phrased as follows.

Forces, as abstract entities, are perceived by imposing test displacements (and their derivatives) at every point $\boldsymbol{x} \in \bar{\Omega}$, in order to characterize them completely in magnitude and direction.

Note that the magnitude does not require the concept of a metric in this context. The magnitude is given by the virtual work (4), which is per definition a metricindependent scalar. This scalar furthermore does not depend on the chosen coordinate representation of $\delta \boldsymbol{\xi}$ and $\mathrm{d} \boldsymbol{F}^{k}$, nor on the parametrization of the appearing integral. This motivates regarding the virtual work as the invariant quantity in mechanics. Conversely, the invariance of the virtual work provides all the transformation rules for the occurring force quantities when a coordinate representation is chosen, or when the parametrization of the integral is changed. The physical units of forces, virtual displacements, and the virtual work are $[\boldsymbol{F}]=\mathrm{N},[\delta \boldsymbol{\xi}]=\mathrm{m}$, and $[\delta W]=\mathrm{Nm}=\mathrm{J}$, respectively. Based on the definition of force, we may now state the first axiom, which is the equilibrium conditions of a body in variational form.

Axiom 1 (principle of virtual work). Let the force measures $\mathrm{d} \boldsymbol{F}^{k}$ contain the totality of forces acting on the body in its deformed configuration $\bar{\Omega}$. For the body to be in equilibrium, the overall virtual work $\delta W_{\text {tot }}$ generated by $\mathrm{d} \boldsymbol{F}^{k}$ has to vanish for all virtual displacements $\delta \xi$ :

$$
\delta W_{\mathrm{tot}}=\sum_{k=0}^{N} \int_{\bar{\Omega}} \frac{\partial^{k}}{\partial \boldsymbol{x}^{k}}(\delta \boldsymbol{\xi}) \mid \mathrm{d} \boldsymbol{F}^{k}=0 \quad \text { for all } \delta \boldsymbol{\xi} .
$$

The principle of virtual work (5) requires the totality of forces acting on the body, which encompasses both the internal and the external forces of $\bar{\Omega}$, as they are defined below. This totality represented by the force measures $\mathrm{d} \boldsymbol{F}^{k}(\boldsymbol{x})$ is obtained by the pointwise summation of the individual force contributions $\mathrm{d} \boldsymbol{F}_{i}^{k}(\boldsymbol{x})$, since forces as linear functionals are additive. Furthermore, (5) has to hold for arbitrary virtual displacement fields $\delta \xi$. This arbitrariness implies that the virtual work also has to vanish for all suitably regular subbodies.

Definition 2 (internal and external forces). Let $K$ be a subsystem of $\bar{\Omega}$, that is, $K \subseteq \bar{\Omega}$. We call $\boldsymbol{F}^{\mathrm{i}}$ an internal force of $K$ if it is exerted from material points 
$\boldsymbol{x} \in K$ on other material points $\boldsymbol{y} \in K$. We call $\boldsymbol{F}^{\mathrm{e}}$ an external force of $K$ if it is exerted from the environment of $K$ on some material points $\boldsymbol{x} \in K$.

Internal forces describe the force interactions among the points $x \in K$ exclusively, whereas external forces act from outside on the points $\boldsymbol{x} \in K$. The classification into internal and external forces therefore depends strongly on the considered subsystem. An external force $\boldsymbol{F}$ of $K \subset \bar{\Omega}$, which acts from a subset $B \subset \bar{\Omega} \backslash K$ on some material points in $K$, is an internal force of any subsystem $C \cup K$ with $C \supseteq B$. This definition is very classical and strongly related to mechanical modeling. The same classification can be found for instance in [Hellinger 1914, pp. 637-638] ${ }^{10}$ or [Germain 1973b] ${ }^{11}$. One must bear in mind that every mechanical interaction which one desires to model, in order to predict an observation, has to be introduced explicitly in the mathematical framework of a mechanical theory. Based on the definition of the internal forces, we may now state the second axiom.

Axiom 2 (variational law of interaction ${ }^{12}$ ). Let $\delta W^{\mathrm{i}}$ denote the virtual work generated by the totality of internal forces measures $\mathrm{d} \boldsymbol{F}^{\mathrm{i}, k}$ of an arbitrary subsystem $K \subseteq \bar{\Omega}$. It then holds that $\delta W^{\mathrm{i}}$ vanishes under each rigid virtual displacement field $\delta \xi_{\text {rig }}$ as specified in (3):

$$
\delta W^{\mathrm{i}}=\sum_{k=0}^{N} \int_{K} \frac{\partial^{k}}{\partial \boldsymbol{x}^{k}}(\delta \boldsymbol{\xi}) \mid \mathrm{d} \boldsymbol{F}^{\mathrm{i}, k}=0 \quad \text { for all } \delta \boldsymbol{\xi}_{\mathrm{rig}} .
$$

From a mechanical point of view, the variational law of interaction can be interpreted as follows: the totality of internal force measures $\mathrm{d} \boldsymbol{F}^{\mathrm{i}, k}$ of the subsystem $K \subseteq \bar{\Omega}$ is not perceived from the outside when the subsystem as a whole is virtually moved like a rigid body, or in other words, one does not have to work against the total internal force under any virtual rigid body motion. It has to be mentioned that (6) does not give any conditions on the force measures $\mathrm{d} \boldsymbol{F}^{\mathrm{i}, k}$ of order $k>1$.

${ }^{10}$ Also see the translation [Eugster and dell'Isola 2017b, p. 11]: "Thereby primarily the [following] difference must be clarified, if the force effects are external, i.e., [the effects] have their cause in the relation to media and sources of effects located outside the considered medium (long-range forces, pressures at the boundary and such like), or internal, i.e., [the effects] are based on the material constitution of the particular medium and the mutual effects of the particles thereof."

11 "The various "forces" which act on the mechanical system are divided in a very classical way into two classes: external forces which represent the dynamical effects on $S$ due to the interaction with other systems which have no common part with $S$, and internal forces which represent the mutual dynamical effects of subsystems of $S$; for instance, if $S_{i}$ and $S_{j}$ are two disjoint subsystems of $S$, the action of $S_{i}$ on $S_{j}$ represents "external forces" acting on $S_{j}$, but "internal forces" if one considers the system $S$ itself."

${ }^{12}$ Denoted by Germain [1973b] as axiom of power of internal forces. We prefer to call it variational law of interaction as it is the variational version of the law of interaction proposed by Glocker [2001, Chapter 2]. 
In contrast to the first axiom, the variational law of interaction requires a metric to extract from it the rigid virtual displacement fields $\delta \xi_{\text {rig }}$, for which the variations are evaluated. By using their specific parametrization (3), the universal quantifier in (6) implies that the variations have to be carried out independently for the two kinematic quantities $\delta \boldsymbol{r}_{O}$ and $\delta \boldsymbol{\phi}$. This leads to the resultant forces as the quantities dual to $\delta \boldsymbol{r}_{O}$, but also to the first occurrence of (resultant) moments as the quantities dual to $\delta \phi$. The fact that precisely three equations are obtained for the resultant forces, and another three for the moments, is attributed to the specific form (3) of the rigid virtual displacement field in $\mathbb{E}^{3}$ and would be different if another metric space would have been chosen for the embedding. Note that the moments always lead back to the variational law of interaction (6) in our approach, as no metric is needed in the principle of virtual work (5), nor in the representation of forces (4).

\section{From Piola's micro-macro identification to the virtual work of stress}

Equation (4) shows us the wide variety of possible force interactions in a continuum. In most variational formulations of continuum mechanics, as, e.g., [Hellinger 1914; Germain 1972; 1973a; 1986; Salençon 2000; Salençon 2001], only distinct subsets of these force representations are postulated for the internal and external virtual work. In this section, we show how these virtual work contributions of the classical continuum can be derived using Piola's micro-macro identification procedure.

Integration always consists of a dissection of a region into simple elements, followed by a limit process with a refinement of these elements. We sketch this process for the Riemann integral over the body volume with a dissection of the deformed configuration $\Omega$ into cuboids and an approximation from the inside. As a sort of a lower Darboux sum, an approximation from the inside sums the contributions of the inner cuboids, i.e., the cuboids lying completely within the domain $\bar{\Omega}$; see Figure 2. In what follows, we will denote the position vector of the center of a cuboid $i j k$ with respect to the $I$-system by ${ }_{I} \boldsymbol{x}_{i j k}=\left(x_{i}, y_{j}, z_{k}\right)^{\mathrm{T}}$. The indices $i, j, k$ appearing in the subsequent sums range always such that all inner cuboids are taken into account. Without loss of generality, we assume all the infinitesimal volume elements to be of the same size $\Delta V=\Delta x \Delta y \Delta z$, where $\Delta x, \Delta y, \Delta z$ denote the lengths of the corresponding edges. Accordingly, the volume integral over the body $\Omega$ of a function $w(\boldsymbol{x})$ is obtained when the limit

$$
\int_{\Omega} w(\boldsymbol{x}) \mathrm{d} v=\lim _{\Delta V \rightarrow 0} \sum_{i, j, k} w\left(x_{i}, y_{j}, z_{k}\right) \Delta V
$$

does exist.

It is now this type of volume integral which we use in the context to interpret Piola's micro-macro identification procedure. Crucial for the following is that the 


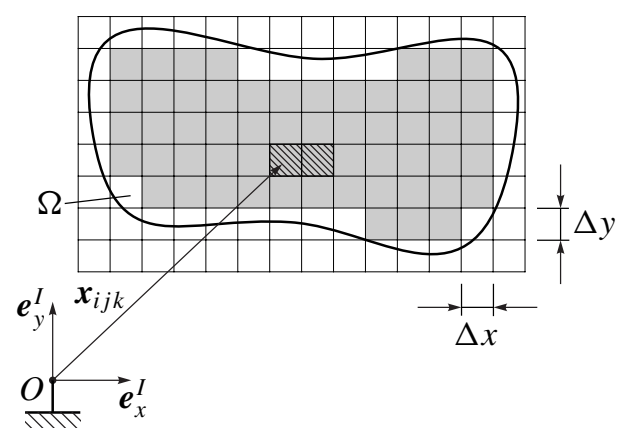

Figure 2. Dissection of the deformed configuration $\Omega$ into cuboids and approximation of the Riemann integral from the inside. The inner cuboids lying completely within the domain $\bar{\Omega}$ are colored in gray.

discrete approximation of the body by finite volume elements can already be considered as a mechanical model, i.e., the micromodel. The identification procedure is then performed as follows.

- The virtual work contributions $\Delta W$ are stated for the force interactions that occur (or are modeled) in the discrete micromodel.

- The virtual displacement of a discrete volume element $i j k$ is identified with the continuous virtual displacement field evaluated at an arbitrary point inside the volume element - for instance $\delta \boldsymbol{\xi}\left(\boldsymbol{x}_{i j k}\right)$ when it is evaluated at the center point $\boldsymbol{x}_{i j k}$.

- In the limit of vanishing volume elements $\Delta V \rightarrow 0$, the virtual work contribution of the continuous macromodel $\delta W$ is identified with the virtual work of the discrete micromodel $\Delta W$, i.e., $\delta W=\lim _{\Delta V \rightarrow 0} \Delta W$.

Obviously, the same procedure can also be applied for lower dimensional integrations, to obtain force interactions on lines or surfaces.

What now follows for the external forces can be found similarly in [Piola 1848, $\S \S 31-32]^{13}$, despite the fact that Piola uses a reference configuration to address the material points. One kind of force interaction that we allow to be exerted from the environment on the body, i.e., as an external force, leads to the virtual work contribution

$$
\Delta W_{v}^{\mathrm{e}}=\sum_{i, j, k} \delta \xi\left(\boldsymbol{x}_{i j k}\right) \cdot \Delta \boldsymbol{F}_{v}\left(\boldsymbol{x}_{i j k}\right)=\sum_{i, j, k} \delta \boldsymbol{\xi}\left(\boldsymbol{x}_{i j k}\right) \cdot \frac{\Delta \boldsymbol{F}_{v}\left(\boldsymbol{x}_{i j k}\right)}{\Delta V} \Delta V
$$

${ }^{13}$ An English translation is given in [Piola 2014, Chapter 1, pp. 51-55]. 
of the discrete micromodel. Remark that the virtual work of a single cuboid is obtained by the duality pairing between the virtual displacement $\delta \boldsymbol{\xi}\left(\boldsymbol{x}_{i j k}\right)$ and the force $\Delta \boldsymbol{F}_{v}\left(\boldsymbol{x}_{i j k}\right)$, which can be considered as a force in the sense of Newton. The virtual work $\delta W_{v}^{\mathrm{e}}$ of the external force effect appearing in the continuous macromodel is then identified with the micromodel by

$$
\delta W_{v}^{\mathrm{e}}=\lim _{\Delta V \rightarrow 0} \Delta W_{v}^{\mathrm{e}}=\int_{\Omega} \delta \xi \cdot \boldsymbol{f}_{v} \mathrm{~d} v,
$$

where the existence of the limit

$$
\boldsymbol{f}_{v}(\boldsymbol{x}):=\lim _{\Delta V \rightarrow 0} \frac{\Delta \boldsymbol{F}_{v}\left(\boldsymbol{x}_{i j k}\right)}{\Delta V}
$$

has to be assumed. Equation (10) is nothing else but the definition of a volume force with unit $\left[f_{v}\right]=\mathrm{N} / \mathrm{m}^{3}$.

The forces exerted by the environment on the body via the surface of the deformed configuration $\partial \Omega$ can be modeled in a similar way. This leads then to a surface force $f_{a}$ with unit $\left[f_{a}\right]=\mathrm{N} / \mathrm{m}^{2}$ and to the corresponding virtual work contribution $\delta \boldsymbol{\xi} \cdot \boldsymbol{f}_{a}$, such that the virtual work of external forces for the continuum is of the form

$$
\delta W^{\mathrm{e}}=\int_{\Omega} \delta \boldsymbol{\xi} \cdot \boldsymbol{f}_{v} \mathrm{~d} v+\int_{\partial \Omega} \delta \boldsymbol{\xi} \cdot \boldsymbol{f}_{a} \mathrm{~d} a
$$

We could also introduce further virtual work contributions of external force effects that are acting on surfaces, lines, or even points inside the body.

On the micro level of volume elements, one may also introduce force effects between the individual volume elements. This is exactly the idea, which enables us to give a verbal definition of stress on the discrete micro level of infinitesimal volume elements in the sense of Boltzmann.

Definition 3 (stress). We define stress as the internal force effects of the body that model the mutual force interactions between neighboring infinitesimal volume elements sharing the same surfaces.

We want to mention that this definition explicitly excludes the interaction between cuboids sharing the same edges and wedge points. The upcoming derivation shows how the three stress vectors of the continuous macro level emerge in the virtual work from Definition 3 together with Piola's micro-macro identification procedure.

In a first step, we consider two neighboring cuboids with coordinates ${ }_{I} \boldsymbol{x}_{i j k}=$ $\left(x_{i}, y_{j}, z_{k}\right)^{\mathrm{T}}$ and ${ }_{I} \boldsymbol{x}_{(i+1) j k}=\left(x_{i+1}, y_{j}, z_{k}\right)^{\mathrm{T}}$, which are aligned in the $\boldsymbol{e}_{x}^{I}$-direction as depicted in Figure 2 as hatched elements. As all volume elements are of the same size, it holds that $x_{i+1}=x_{i}+\Delta x$. Figure 3 shows a close-up of these two cuboids together with their virtual displacements $\delta \xi$ and their force interactions 


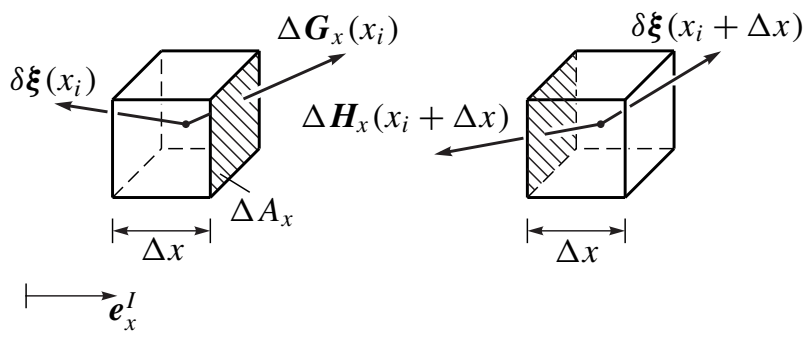

Figure 3. Force interaction between two neighboring volume elements aligned in the $\boldsymbol{e}_{x}^{I}$-direction.

$\Delta \boldsymbol{G}_{x}$ and $\Delta \boldsymbol{H}_{x}$. For the sake of brevity, we omit in the figures as well as in the upcoming formulas the dependence of $\delta \boldsymbol{\xi}, \Delta \boldsymbol{G}_{x}$, and $\Delta \boldsymbol{H}_{x}$ on the coordinates $y_{j}$ and $z_{k}$. The discrete virtual work contribution of the two adjacent cuboids by the force increments $\Delta \boldsymbol{G}_{x}$ and $\Delta \boldsymbol{H}_{x}$ is

$$
\Delta W^{\mathrm{s}}=\delta \boldsymbol{\xi}\left(x_{i}\right) \cdot \Delta \boldsymbol{G}_{x}\left(x_{i}\right)+\delta \boldsymbol{\xi}\left(x_{i}+\Delta x\right) \cdot \Delta \boldsymbol{H}_{x}\left(x_{i}+\Delta x\right)
$$

with $\Delta \boldsymbol{G}_{x}$ being the force with unit $\left[\Delta \boldsymbol{G}_{x}\right]=\mathrm{N}$ exerted from the right cuboid on the left cuboid and $\Delta \boldsymbol{H}_{x}$ being the force with unit $\left[\Delta \boldsymbol{H}_{x}\right]=\mathrm{N}$ exerted from the left cuboid on the right cuboid. To relate the forces $\Delta \boldsymbol{G}_{x}$ and $\Delta \boldsymbol{H}_{x}$ in (12) to each other, we introduce forces $\Delta \boldsymbol{F}_{x}$ and $\Delta \boldsymbol{C}_{x}$ such that

$$
\Delta \boldsymbol{G}_{x}\left(x_{i}\right)=\Delta \boldsymbol{F}_{x}\left(x_{i}\right)+\Delta \boldsymbol{C}_{x}\left(x_{i}\right) \text { and } \Delta \boldsymbol{H}_{x}\left(x_{i}+\Delta x\right)=-\Delta \boldsymbol{F}_{x}\left(x_{i}\right) .
$$

Note that we intentionally violate here the principle of action and reaction by the force $\Delta \boldsymbol{C}_{x}$ : the principle of action and reaction does not constitute an independent axiom in our approach, but is contained in the variational law of interaction. Consequently, we do not apply action equals reaction by setting $\Delta \boldsymbol{C}_{x}=0$, but leave it to the variational law of interaction on the macro level to decide later whether the force $\Delta \boldsymbol{C}_{x}$ is needed in (13). With the help of (13), the virtual work contribution (12) of the two adjacent cuboids becomes

$$
\begin{aligned}
\Delta W^{\mathrm{s}} & =\delta \boldsymbol{\xi}\left(x_{i}\right) \cdot \Delta \boldsymbol{C}_{x}\left(x_{i}\right)+\delta \boldsymbol{\xi}\left(x_{i}\right) \cdot \Delta \boldsymbol{F}_{x}\left(x_{i}\right)-\delta \boldsymbol{\xi}\left(x_{i}+\Delta x\right) \cdot \Delta \boldsymbol{F}_{x}\left(x_{i}\right) \\
& =\delta \boldsymbol{\xi}\left(x_{i}\right) \cdot \Delta \boldsymbol{C}_{x}\left(x_{i}\right)-\left(\delta \boldsymbol{\xi}\left(x_{i}+\Delta x\right)-\delta \boldsymbol{\xi}\left(x_{i}\right)\right) \cdot \Delta \boldsymbol{F}_{x}\left(x_{i}\right) \\
& =\delta \boldsymbol{\xi}\left(x_{i}\right) \cdot \Delta \boldsymbol{C}_{x}\left(x_{i}\right)-\left(\delta \boldsymbol{\xi}\left(x_{i}+\Delta x\right)-\delta \boldsymbol{\xi}\left(x_{i}\right)\right) \frac{1}{\Delta x} \cdot \Delta x \Delta \boldsymbol{F}_{x}\left(x_{i}\right) .
\end{aligned}
$$

We denote by $\Delta A_{x}:=\Delta y \Delta z$ the surface element that is shared by the adjacent cuboids and rewrite (14) as

$$
\Delta W^{\mathrm{s}}=\left(\delta \boldsymbol{\xi}\left(x_{i}\right) \cdot \frac{\Delta \boldsymbol{C}_{x}\left(x_{i}\right)}{\Delta V}-\frac{\delta \boldsymbol{\xi}\left(x_{i}+\Delta x\right)-\delta \boldsymbol{\xi}\left(x_{i}\right)}{\Delta x} \cdot \frac{\Delta \boldsymbol{F}_{x}\left(x_{i}\right)}{\Delta A_{x}}\right) \Delta V .
$$



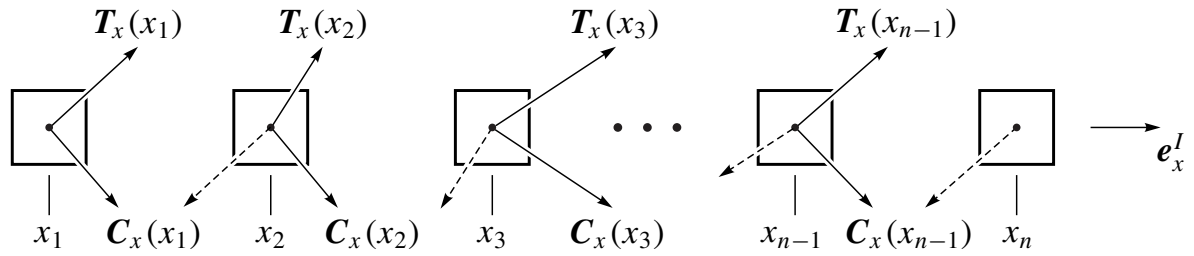

Figure 4. Mutual force interactions between the members of the row of inner cuboids in the direction $\boldsymbol{e}_{x}^{I}$ at the position $y_{j}, z_{k}$.

Furthermore, we introduce in (15) the abbreviations

$$
\begin{aligned}
\boldsymbol{C}_{x}\left(x_{i}\right) & :=\frac{\Delta \boldsymbol{C}_{x}\left(x_{i}\right)}{\Delta V}, \\
\boldsymbol{T}_{x}\left(x_{i}\right) & :=\frac{\Delta \boldsymbol{F}_{x}\left(x_{i}\right)}{\Delta A_{x}}, \\
D_{x} \delta \boldsymbol{\xi}\left(x_{i}\right) & :=\frac{\delta \boldsymbol{\xi}\left(x_{i}+\Delta x\right)-\delta \boldsymbol{\xi}\left(x_{i}\right)}{\Delta x},
\end{aligned}
$$

where $\boldsymbol{C}_{x}$ is the incremental volume force with unit $\left[\boldsymbol{C}_{x}\right]=\mathrm{N} / \mathrm{m}^{3}, \boldsymbol{T}_{x}$ is the incremental stress vector with unit $\left[\boldsymbol{T}_{x}\right]=\mathrm{N} / \mathrm{m}^{2}$, and $D_{x} \delta \boldsymbol{\xi}$ is the dimensionless differential quotient. Inserting the abbreviations (16) in (15), we obtain

$$
\Delta W^{\mathrm{s}}=\delta \boldsymbol{\xi}\left(x_{i}\right) \cdot \boldsymbol{C}_{x}\left(x_{i}\right) \Delta V-D_{x} \delta \boldsymbol{\xi}\left(x_{i}\right) \cdot \boldsymbol{T}_{x}\left(x_{i}\right) \Delta V .
$$

Equation (17) is the virtual work contribution of the mutual force interaction of two neighboring cuboids, which is the interaction of the two cuboids by internal short-range forces. As such, $\Delta W^{\mathrm{s}}$ will contribute in the limit to the internal virtual work $\delta W^{\mathrm{i}}$ in (6).

Figure 4 depicts the row of inner cuboids aligned in the $\boldsymbol{e}_{x}^{I}$-direction at the position $y_{j}, z_{k}$, together with all mutual force interactions that occur between each pair of neighboring cuboids. By adding all these force interactions to $\Delta W^{\mathrm{s}}$ in (17), we get the virtual work contribution of this entire row as

$$
\begin{aligned}
\Delta W^{\mathrm{s}}=\sum_{i=1}^{n-1} \delta \boldsymbol{\xi}\left(x_{i}, y_{j}, z_{k}\right) \cdot \boldsymbol{C}_{x}\left(x_{i}, y_{j}, z_{k}\right) \Delta V \\
\\
\quad-D_{x} \delta \boldsymbol{\xi}\left(x_{i}, y_{j}, z_{k}\right) \cdot \boldsymbol{T}_{x}\left(x_{i}, y_{j}, z_{k}\right) \Delta V .
\end{aligned}
$$

Summation of all the cuboid rows in the $\boldsymbol{e}_{x}^{I}$-direction leads to

$$
\Delta W^{\mathrm{s}}=\sum_{i, j, k}\left(\delta \boldsymbol{\xi} \cdot \boldsymbol{C}_{x}\right)_{i j k} \Delta V-\left(D_{x} \delta \boldsymbol{\xi} \cdot \boldsymbol{T}_{x}\right)_{i j k} \Delta V,
$$

which corresponds to a summation over all inner cuboids of the deformed configuration $\Omega$. The force interactions $\left(\boldsymbol{C}_{y}, \boldsymbol{T}_{y}\right)$ and $\left(\boldsymbol{C}_{z}, \boldsymbol{T}_{z}\right)$ to the neighboring cuboids 
in the $y$-and $z$-directions contribute in a similar way. Consequently, the entire virtual work of stress of the discrete micromodel is

$$
\begin{aligned}
\Delta W^{\mathrm{S}}=\sum_{i, j, k}\left(\delta \boldsymbol{\xi} \cdot \boldsymbol{C}_{x}+\delta \boldsymbol{\xi} \cdot \boldsymbol{C}_{y}+\delta \boldsymbol{\xi} \cdot \boldsymbol{C}_{z}\right)_{i j k} \Delta V \\
-\left(D_{x} \delta \boldsymbol{\xi} \cdot \boldsymbol{T}_{x}+D_{y} \delta \boldsymbol{\xi} \cdot \boldsymbol{T}_{y}+D_{z} \delta \boldsymbol{\xi} \cdot \boldsymbol{T}_{z}\right)_{i j k} \Delta V,
\end{aligned}
$$

which models all mutual force interactions between the neighboring inner cuboids.

The virtual work $\delta W^{\mathrm{s}}$ of the mutual force interactions on the macro level is then identified with the virtual work of the micromodel (20) by taking the limit

$$
\delta W^{\mathrm{s}}=\lim _{\Delta V \rightarrow 0} \Delta W^{\mathrm{s}},
$$

which requires the limits of the objects in (16) to exist. We denote them by

$$
\begin{aligned}
\boldsymbol{c}_{x}\left({ }_{I} \boldsymbol{x}\right) & :=\lim _{\Delta V \rightarrow 0} \boldsymbol{C}_{x}\left(x_{i}, y_{j}, z_{k}\right), \\
\boldsymbol{t}_{x}\left({ }_{I} \boldsymbol{x}\right) & :=\lim _{\Delta A_{x} \rightarrow 0} \boldsymbol{T}_{x}\left(x_{i}, y_{j}, z_{k}\right), \\
\delta \boldsymbol{\xi}_{, x}\left({ }_{I} \boldsymbol{x}\right) & :=\lim _{\Delta x \rightarrow 0} D_{x} \delta \boldsymbol{\xi}\left(x_{i}, y_{j}, z_{k}\right) .
\end{aligned}
$$

Similar to (16), $\boldsymbol{c}_{x}$ is a volume force with unit $\left[\boldsymbol{c}_{x}\right]=\mathrm{N} / \mathrm{m}^{3}, \boldsymbol{t}_{x}$ is the stress vector with unit $\left[\boldsymbol{t}_{x}\right]=\mathrm{N} / \mathrm{m}^{2}$, and $\delta \boldsymbol{\xi}_{, x}$ is the dimensionless partial derivative of $\delta \boldsymbol{\xi}$ with respect to $x$. The corresponding contributions in the $y$ - and $z$-directions are denoted accordingly. Using (20) and (22) and defining

$$
\boldsymbol{c}:=\boldsymbol{c}_{x}+\boldsymbol{c}_{y}+\boldsymbol{c}_{z},
$$

we can identify the virtual work of the continuous macromodel as

$$
\delta W^{\mathrm{s}}=\int_{\Omega}\left(\delta \boldsymbol{\xi} \cdot \boldsymbol{c}-\delta \boldsymbol{\xi}_{, x} \cdot \boldsymbol{t}_{x}-\delta \boldsymbol{\xi}_{, y} \cdot \boldsymbol{t}_{y}-\delta \boldsymbol{\xi}_{, z} \cdot \boldsymbol{t}_{z}\right) \mathrm{d} v,
$$

where $\mathrm{d} v$ denotes the volume element in the deformed configuration. In addition to the term $\delta \xi \cdot c$, the stress vectors $\boldsymbol{t}_{i}$ of the three orthogonal spatial directions $i=x, y, z$ contribute to $\delta W^{\mathrm{s}}$, paired with the corresponding partial derivatives $\delta \boldsymbol{\xi}_{, i}$.

The reason to take the limits like in (22) and not in any other form is motivated as follows. If we assume $\Delta \boldsymbol{C}_{x}=0$, then $\Delta \boldsymbol{G}_{x}$ and $\Delta \boldsymbol{H}_{x}$ in (13) can be interpreted as two mutual force distributions, equal in size and opposite in direction, which are infinitesimally shifted against each other. If we would factor out $\Delta V$ and already take the limit $\Delta V \rightarrow 0$ in the second line of (14), the volume force $\Delta \boldsymbol{F}_{x} / \Delta V$, paired with the vanishing difference $\delta \xi\left(x_{i}+\Delta x\right)-\delta \xi\left(x_{i}\right)$, would have to be infinitely large to produce a nonvanishing contribution to the integral (" $0 \cdot \infty$ "). The mere difference $\delta \xi\left(x_{i}+\Delta x\right)-\delta \boldsymbol{\xi}\left(x_{i}\right)$ alone is not strong enough to keep bounded volume forces for $\Delta V \rightarrow 0$ in the integral. However, by using the differential quotient $(1 / \Delta x)\left[\delta \xi\left(x_{i}+\Delta x\right)-\delta \xi\left(x_{i}\right)\right]$ as in the third line of (14), the aforementioned 
difference is strengthened by the division with $\Delta x$ and a nonzero expression is obtained. At the same time, the volume force $\Delta \boldsymbol{F}_{x} / \Delta V$, which tends to infinity for $\Delta V \rightarrow 0$, has to be moderated to a surface force $\Delta \boldsymbol{F}_{x} / \Delta A_{x}$, which, after the limit process, is called the stress vector $\boldsymbol{t}_{x}$.

The choice of a particular cartesian parametrization of the deformed configuration $\Omega$ in the formulation (24) was inevitable. In order to get rid of this particular choice, we bring (24) into a coordinate-free form, in which the stress tensor emerges as a Euclidean second-order tensor. Using the cartesian coordinates $(x, y, z)$ with the orthonormal basis vectors $\left(\boldsymbol{e}_{x}^{I}, \boldsymbol{e}_{y}^{I}, \boldsymbol{e}_{z}^{I}\right)$, the partial derivative with respect to the position vector $\boldsymbol{x}$ is defined as

$$
\frac{\partial \delta \boldsymbol{\xi}}{\partial \boldsymbol{x}}:=\delta \boldsymbol{\xi}_{, x} \otimes \boldsymbol{e}_{x}^{I}+\delta \boldsymbol{\xi}_{, y} \otimes \boldsymbol{e}_{y}^{I}+\delta \boldsymbol{\xi}_{, z} \otimes \boldsymbol{e}_{z}^{I} .
$$

The partial derivatives $\delta \boldsymbol{\xi}_{, i}$ of the virtual displacement field with respect to the coordinates $i=x, y, z$ can therefore be extracted by contracting the last tensor slot of the partial derivative with the basis vectors $\boldsymbol{e}_{i}^{I}$ :

$$
\delta \boldsymbol{\xi}_{, x}=\frac{\partial \delta \boldsymbol{\xi}}{\partial \boldsymbol{x}} \cdot \boldsymbol{e}_{x}^{I}, \quad \delta \boldsymbol{\xi}_{, y}=\frac{\partial \delta \boldsymbol{\xi}}{\partial \boldsymbol{x}} \cdot \boldsymbol{e}_{y}^{I}, \quad \delta \boldsymbol{\xi}_{, z}=\frac{\partial \delta \boldsymbol{\xi}}{\partial \boldsymbol{x}} \cdot \boldsymbol{e}_{z}^{I},
$$

where the dot denotes the contraction. For a second-order tensor $A \in \mathbb{E}^{3} \otimes \mathbb{E}^{3}$ and vectors $\boldsymbol{u}, \boldsymbol{v} \in \mathbb{E}^{3}$, the relation $(\boldsymbol{A} \cdot \boldsymbol{v}) \cdot \boldsymbol{u}=\boldsymbol{u} \cdot(\boldsymbol{A} \cdot \boldsymbol{v})=\boldsymbol{A}:(\boldsymbol{u} \otimes \boldsymbol{v})$ holds. Using this relation together with (26), the terms involving the stress vectors $\boldsymbol{t}_{i}$ in (24) are reformulated as

$$
\begin{aligned}
\delta \boldsymbol{\xi}_{, x} \cdot \boldsymbol{t}_{x}+\delta \boldsymbol{\xi}_{, y} \cdot \boldsymbol{t}_{y}+\delta \boldsymbol{\xi}_{, z} \cdot \boldsymbol{t}_{z} & \\
& =\left(\frac{\partial \delta \boldsymbol{\xi}}{\partial \boldsymbol{x}} \cdot \boldsymbol{e}_{x}^{I}\right) \cdot \boldsymbol{t}_{x}+\left(\frac{\partial \delta \boldsymbol{\xi}}{\partial \boldsymbol{x}} \cdot \boldsymbol{e}_{y}^{I}\right) \cdot \boldsymbol{t}_{y}+\left(\frac{\partial \delta \boldsymbol{\xi}}{\partial \boldsymbol{x}} \cdot \boldsymbol{e}_{z}^{I}\right) \cdot \boldsymbol{t}_{z}=\frac{\partial \delta \boldsymbol{\xi}}{\partial \boldsymbol{x}}: \boldsymbol{\sigma},
\end{aligned}
$$

where the stress tensor $\sigma$ emerges as being the second-order Euclidean tensor field

$$
\boldsymbol{\sigma}(\boldsymbol{x}):=\boldsymbol{t}_{x}(\boldsymbol{x}) \otimes \boldsymbol{e}_{x}^{I}+\boldsymbol{t}_{y}(\boldsymbol{x}) \otimes \boldsymbol{e}_{y}^{I}+\boldsymbol{t}_{z}(\boldsymbol{x}) \otimes \boldsymbol{e}_{z}^{I} .
$$

The stress tensor field (28) as the tensor field over the deformed configuration $\Omega$ is called Cauchy stress. Using (27), the virtual work of the stress (24) can now be written in the form

$$
\delta W^{\mathrm{s}}=\int_{\Omega}\left(\delta \boldsymbol{\xi} \cdot \boldsymbol{c}-\frac{\partial \delta \boldsymbol{\xi}}{\partial \boldsymbol{x}}: \boldsymbol{\sigma}\right) \mathrm{d} v .
$$

Comparing the virtual work (29) with the possible force representations (4), we immediately recognize that the stress contributes with volume measures of order zero, $\mathrm{d} \boldsymbol{F}^{0}=\boldsymbol{c} \mathrm{d} v$, and order one, $\mathrm{d} \boldsymbol{F}^{1}=-\sigma \mathrm{d} v$. Even though the derivation of (29) makes use of cartesian coordinates for the parametrization of the domain $\Omega$, the virtual work of the stress (29) includes only Euclidean vector and tensor 
fields together with tensor operations. Consequently, (29) is independent of any choice of coordinates and can therefore be claimed to be coordinate-free. Note that definitions (25) and (28) rely on cartesian coordinates. For curvilinear coordinates, these definitions have to be revisited and require the concepts of coand contravariant basis vectors [Başar and Weichert 2000; Green and Zerna 1954; Ogden 1997], which goes beyond the scope of this paper. However, and herein lies the strength of a variational formulation, since the virtual work is invariant with respect to the parametrization of the appearing integral, (24) can also be applied to curvilinear coordinates. Without introducing partial derivatives or divergence operators for curvilinear coordinates, we will demonstrate in the Appendix how to derive the local equilibrium equations in cylindrical coordinates, by starting from (24) instead of (29). For spaces more general than $\mathbb{E}^{3}$, as for example manifolds, the partial derivative in (29) is not defined anymore and has to be exchanged with a covariant derivative, as discussed in [Eugster 2015a; 2015b; Segev 1986].

\section{Symmetry of the stress tensor}

In this section, we prove the symmetry of the stress tensor. Our approach basically follows the strategy proposed by Germain $[1972 ; 1973 b]$ and can be regarded as a detailed version of it. According to Definition 3, stresses contribute to the internal forces of the body. If now the stresses are the only internal force contributions, they also form the totality of internal forces and as such they have to satisfy the variational law of interaction (Axiom 2) in their entirety. The following proof is nothing else but the evaluation of the variational law of interaction for the stresses. Note that the proof will be carried out by exclusively using the variational law of interaction, meaning that external forces are nowhere needed in the argumentation up to the point at which the existence of the stress tensor is addressed. We start with the variational law of interaction (6), by which the virtual work $\delta W^{\mathrm{s}}$ of the stress has to satisfy

$$
\delta W^{\mathrm{s}}=0 \quad \text { for all } \delta \boldsymbol{\xi}_{K, \text { rig }}(\boldsymbol{x})= \begin{cases}\delta \boldsymbol{\xi}_{\mathrm{rig}}(\boldsymbol{x}) & \text { for } \boldsymbol{x} \in K \\ 0 & \text { for } \boldsymbol{x} \notin K\end{cases}
$$

for any open set $K \subseteq \Omega$ with rigid virtual displacements $\delta \xi_{\text {rig }}$ as defined in (3). Equation (30) implies additional conditions on the force quantities $\boldsymbol{c}$ and $\boldsymbol{t}_{i}$ in (24), which are elaborated in the following and which will lead to the symmetry conditions in question. Combining (30) and (24) yields

$$
0=\int_{K}\left(\delta \boldsymbol{\xi}_{\mathrm{rig}} \cdot \boldsymbol{c}-\delta \boldsymbol{\xi}_{\mathrm{rig}, x} \cdot \boldsymbol{t}_{x}-\delta \boldsymbol{\xi}_{\mathrm{rig}, y} \cdot \boldsymbol{t}_{y}-\delta \boldsymbol{\xi}_{\mathrm{rig}, z} \cdot \boldsymbol{t}_{z}\right) \mathrm{d} v \quad \text { for all } K \text { and all } \delta \boldsymbol{\xi}_{\mathrm{rig}}
$$


The rigid virtual displacement fields $\delta \boldsymbol{\xi}_{\text {rig }}$ are taken from (3), and their partial derivatives $\delta \xi_{\text {rig, }, i}$ for $i=x, y, z$ are accordingly calculated as

$$
\delta \boldsymbol{\xi}_{\text {rig }}(\boldsymbol{x})=\delta \boldsymbol{r}_{O}+\delta \boldsymbol{\phi} \times \boldsymbol{x} \quad \text { and } \quad \delta \boldsymbol{\xi}_{\mathrm{rig}, i}(\boldsymbol{x})=\delta \boldsymbol{\phi} \times \boldsymbol{x}_{, i} .
$$

Inserting (32) into (31), one obtains the variational condition

$$
\begin{array}{r}
0=\int_{K}\left(\left(\delta \boldsymbol{r}_{O}+\delta \boldsymbol{\phi} \times \boldsymbol{x}\right) \cdot \boldsymbol{c}-\left(\delta \boldsymbol{\phi} \times \boldsymbol{x}_{, x}\right) \cdot \boldsymbol{t}_{x}-\left(\delta \boldsymbol{\phi} \times \boldsymbol{x}_{, y}\right) \cdot \boldsymbol{t}_{y}-\left(\delta \boldsymbol{\phi} \times \boldsymbol{x}_{, z}\right) \cdot \boldsymbol{t}_{z}\right) \mathrm{d} v \\
\text { for all } K, \text { all } \delta \boldsymbol{r}_{O}, \text { and all } \delta \boldsymbol{\phi} .
\end{array}
$$

We use the relation $(\delta \phi \times z) \cdot \boldsymbol{f}=\delta \boldsymbol{\phi} \cdot(\boldsymbol{z} \times \boldsymbol{f})$ to move the quantities $\delta \boldsymbol{r}_{O}$ and $\delta \boldsymbol{\phi}$ in front of the integral and obtain

$0=\delta \boldsymbol{r}_{O} \cdot \int_{K} \boldsymbol{c} \mathrm{d} v+\delta \boldsymbol{\phi} \cdot \int_{K}\left(\boldsymbol{x} \times \boldsymbol{c}-\boldsymbol{x}_{, x} \times \boldsymbol{t}_{x}-\boldsymbol{x}_{, y} \times \boldsymbol{t}_{y}-\boldsymbol{x}_{, z} \times \boldsymbol{t}_{z}\right) \mathrm{d} v$

$$
\text { for all } K \text {, all } \delta \boldsymbol{r}_{O} \text {, and all } \delta \boldsymbol{\phi} \text {. }
$$

The variational law of interaction for the stresses has now been brought into a form which allows one to evaluate the variations $\delta \boldsymbol{r}_{O}$ and $\delta \boldsymbol{\phi}$, and to carry out a subsequent localization step based on the fundamental lemma of calculus of variations.

We start to carry out the variation for all $\delta \boldsymbol{r}_{O}$, while making the specific choice $\delta \boldsymbol{\phi} \equiv 0$. Consequently, (34) reduces to

$$
0=\delta \boldsymbol{r}_{O} \cdot \int_{K} \boldsymbol{c} \mathrm{d} v \quad \text { for all } K \text { and all } \delta \boldsymbol{r}_{O} .
$$

Since (35) has to hold for all $\delta \boldsymbol{r}_{O}$, we obtain

$$
0=\int_{K} c \mathrm{~d} v \text { for all } K
$$

If now $\boldsymbol{c}$ is continuous on a neighborhood $K$ of $\boldsymbol{x}$, then (36) can be localized, which yields

$$
\boldsymbol{c}(\boldsymbol{x})=0 .
$$

The question whether $\boldsymbol{c}$ is continuous at $\boldsymbol{x}$ can not be answered here, i.e., by the variational law of interaction alone. The continuity of $\boldsymbol{c}$ strongly depends on the external forces that are applied on the body, as well as on the constitutive laws that still have to be specified for both the internal and external forces. In other words, the continuity of $\boldsymbol{c}$ involves the principle of virtual work (Axiom 1) because of the external forces, together with the choice of the constitutive laws, and can be checked only after the entire continuum problem has been solved.

In the case that $\boldsymbol{c}$ is continuous at $\boldsymbol{x}$, we have $\boldsymbol{c}=0$ by (37); hence, $\boldsymbol{c}_{x}+\boldsymbol{c}_{y}+\boldsymbol{c}_{z}=0$ by (23). The latter means that the three volume forces $\boldsymbol{c}_{i}$ cancel out each other on the considered volume element and can therefore be disregarded in the ansatz (13). As a consequence, the ansatz (13) simplifies to $\Delta \boldsymbol{G}_{x}\left(x_{i}\right)=\Delta \boldsymbol{F}_{x}\left(x_{i}\right)$ and 
$\Delta \boldsymbol{H}_{x}\left(x_{i}+\Delta x\right)=-\Delta \boldsymbol{F}_{x}\left(x_{i}\right)$, and in the same fashion for the directions $y$ and $z$, with which we have proven that action equals reaction applies for the stress vectors $\boldsymbol{t}_{i}$.

For the upcoming evaluation of (34) with respect to the variations $\delta \boldsymbol{\phi}$, we assume continuity of $\boldsymbol{c}$, that is, $\boldsymbol{c}=0$. As a consequence, the variational problem (34) reduces to

$$
0=\delta \phi \cdot \int_{K}\left(-\boldsymbol{x}_{, x} \times \boldsymbol{t}_{x}-\boldsymbol{x}_{, y} \times \boldsymbol{t}_{y}-\boldsymbol{x}_{, z} \times \boldsymbol{t}_{z}\right) \mathrm{d} v \quad \text { for all } K \text { and all } \delta \phi .
$$

Since (38) has to hold for all $\delta \boldsymbol{\phi}$, we obtain

$$
0=\int_{K}\left(-\boldsymbol{x}_{, x} \times \boldsymbol{t}_{x}-\boldsymbol{x}_{, y} \times \boldsymbol{t}_{y}-\boldsymbol{x}_{, z} \times \boldsymbol{t}_{z}\right) \mathrm{d} v \text { for all } K .
$$

By using the same arguments for the localizing as above, we obtain the symmetry conditions for the stress vectors, which are

$$
\boldsymbol{x}_{, x} \times \boldsymbol{t}_{x}(\boldsymbol{x})+\boldsymbol{x}_{, y} \times \boldsymbol{t}_{y}(\boldsymbol{x})+\boldsymbol{x}_{, z} \times \boldsymbol{t}_{z}(\boldsymbol{x})=0 .
$$

They again hold only for stress vector fields $\boldsymbol{t}_{i}$ which are continuous at $\boldsymbol{x}$. This continuity can again only be checked after the evaluation of the principle of virtual work together with the constitutive laws. A discontinuity appears for instance when an external surface force is applied on a surface inside the body. The same holds true for line forces and point forces. In these cases, continuity can be assumed at least piecewise. Another example showing that the limits of $\boldsymbol{C}_{i}$ and $\boldsymbol{T}_{i}$ in (16) do not even have to exist, at least not uniquely, are the force interactions within a rigid body.

The symmetry of the stress tensor (28) is proven in the same manner as we have shown the symmetry condition for the stress vectors, with the only difference that we start now with the virtual work contribution in the form (29), instead of (24), with vanishing volume force $\boldsymbol{c}=0$. In (29) and (30), the partial derivative of the rigid virtual displacement field with respect to the position vector $\boldsymbol{x}$ is needed, which is by (3) the variation $\delta \boldsymbol{\phi} \times=\delta \tilde{\boldsymbol{\phi}}=\tilde{\boldsymbol{\phi}}_{, \varepsilon}\left(\varepsilon_{0}\right)$ of the skew-symmetric linear map $\tilde{\boldsymbol{\phi}}(\varepsilon)$ as introduced in the text below of (3). Similar to (38), the variational law of interaction implies the condition

$$
0=-\int_{K} \frac{\partial \delta \boldsymbol{\xi}_{\mathrm{rig}}}{\partial \boldsymbol{x}}: \sigma \mathrm{d} v=-\int_{K} \delta \tilde{\boldsymbol{\phi}}: \boldsymbol{\sigma} \mathrm{d} v \quad \text { for all } K \text { and all } \delta \boldsymbol{\phi} .
$$

For a continuous stress tensor field $\sigma,(41)$ can be localized to provide the variational condition

$$
\delta \tilde{\boldsymbol{\phi}}: \sigma=0 \text { for all } \delta \boldsymbol{\phi} .
$$

Now, for $\boldsymbol{S}, \boldsymbol{W} \in \mathbb{E}^{3} \otimes \mathbb{E}^{3}$ with $\boldsymbol{S}$ being symmetric and $\boldsymbol{W}$ being skew-symmetric, it can be shown ${ }^{14}$ that $\boldsymbol{S}: \boldsymbol{W}=0$. Since $\delta \tilde{\boldsymbol{\phi}}$ is skew-symmetric, the only nontrivial

${ }^{14} \boldsymbol{S}: \boldsymbol{W}=\operatorname{tr}\left(\boldsymbol{S}^{\mathrm{T}} \cdot \boldsymbol{W}\right)=\operatorname{tr}\left(\boldsymbol{W} \cdot \boldsymbol{S}^{\mathrm{T}}\right)=\boldsymbol{W}^{\mathrm{T}}: \boldsymbol{S}^{\mathrm{T}}=\boldsymbol{S}^{\mathrm{T}}: \boldsymbol{W}^{\mathrm{T}}=-\boldsymbol{S}: \boldsymbol{W}$ implies $\boldsymbol{S}: \boldsymbol{W}=0$. 
solution for $\sigma$ to guarantee (42) is to be symmetric, which proves the symmetry of the stress tensor

$$
\sigma=\sigma^{\mathrm{T}}
$$

Depending on the specific application, one might choose between the representations (24) and (29) of the virtual work of stress. The same applies for the symmetry conditions, which may be stated either in terms of the stress vectors (40) or in terms of the stress tensor (43). Which one is preferred is often a matter of taste.

For generalized continua as for instance micromorphic continua of degree one [Germain 1973b], the stress tensor obtained from Definition 3 corresponds to the socalled intrinsic stress, which is always symmetric. By the introduction of additional degrees of freedom, as used to describe some microstructure, the nonsymmetric microstress tensor appears as a coupling stress between the micro and the macro levels. ${ }^{15}$ Since the sum of these two stress tensors appears in relation with the surface forces, this sum is commonly called the Cauchy stress tensor, which is in general a nonsymmetric second-order tensor.

\section{Transformation properties of stress}

As mentioned in Section 3, the invariance of the virtual work provides the required transformation properties of the appearing force quantities, when different integral parametrizations or coordinate representations are used. Applying this invariance to the virtual work of the stress (24) or (29), various stress representations can be derived. Without the notion of real work or power, we are able to obtain the work conjugate stress representations [Başar and Weichert 2000; Macvean 1968] from the virtual work alone. Since there is a wealth of possible stress and coordinate representations, we show here just a selection of three important transformation properties. The first is a reparametrization of the integral in (24) with curvilinear coordinates. The second is a reparametrization of the integral in (29) with respect to a new set of position vectors addressing a reference configuration. The last transformation property is obtained for the coordinate representation of (29) when using cartesian coordinates only. All transformation properties are derived for continuous fields, i.e., for $c=0$, with the virtual work contribution of the stress

$$
\delta W^{\mathrm{s}}=-\int_{\Omega}\left(\delta \boldsymbol{\xi}_{, x} \cdot \boldsymbol{t}_{x}+\delta \boldsymbol{\xi}_{, y} \cdot \boldsymbol{t}_{y}+\delta \boldsymbol{\xi}_{, z} \cdot \boldsymbol{t}_{z}\right) \mathrm{d} v=-\int_{\Omega} \frac{\partial \delta \boldsymbol{\xi}}{\partial \boldsymbol{x}}: \boldsymbol{\sigma} \mathrm{d} v,
$$

satisfying the symmetry conditions (40) and (43).

The first integral in (44) is formulated in the deformed configuration and parametrized by cartesian coordinates ${ }_{I} \boldsymbol{x}=(x, y, z)^{\mathrm{T}}$. For a set of curvilinear coordinates

${ }^{15}$ Note that since in this context the micro level is also described by a continuous field, it has to be understood in a way different from the micro level as used in this paper. 
$(p, q, r)^{\mathrm{T}}$ as, e.g., cylindrical coordinates, there exists at least a local invertible map $\varphi$ relating the cartesian with the curvilinear coordinates by

$$
{ }_{I} \boldsymbol{x}=\boldsymbol{\varphi}(p, q, r)=\left(\begin{array}{c}
x(p, q, r) \\
y(p, q, r) \\
z(p, q, r)
\end{array}\right) \quad \text { and } \quad\left(\begin{array}{c}
p \\
q \\
r
\end{array}\right)=\boldsymbol{\varphi}^{-1}\left({ }_{I} \boldsymbol{x}\right)=\left(\begin{array}{c}
p\left({ }_{I} \boldsymbol{x}\right) \\
q\left({ }_{I} \boldsymbol{x}\right) \\
r\left({ }_{I} \boldsymbol{x}\right)
\end{array}\right) .
$$

Note that curvilinear coordinates cannot be related to a position vector $\boldsymbol{x}$ in the sense of (1), where each of the coordinates in ${ }_{I} x$ corresponds to one of the components of the position vector $\boldsymbol{x}$. The volume element in (44) can be expressed in new coordinates as

$\mathrm{d} v=\mathrm{d} x \mathrm{~d} y \mathrm{~d} z=J \mathrm{~d} p \mathrm{~d} q \mathrm{~d} r=J \mathrm{~d} \hat{v}$

$$
\text { with } J=\operatorname{det}(D \varphi)(p, q, r) \text { and } \mathrm{d} \hat{v}=\mathrm{d} p \mathrm{~d} q \mathrm{~d} r,
$$

where $D \varphi$ denotes the Jacobian matrix containing the partial derivatives of $\varphi$. To still integrate over the same set in the Euclidean space, the domain of integration transforms to $\widehat{\Omega}=\varphi^{-1}(\Omega)$. Furthermore, we introduce the virtual displacement field in curvilinear coordinates $\delta \hat{\boldsymbol{\xi}}$, such that the identity

$$
\delta \boldsymbol{\xi}\left({ }_{I} \boldsymbol{x}\right)=\delta \hat{\boldsymbol{\xi}}\left(\boldsymbol{\varphi}^{-1}\left({ }_{I} \boldsymbol{x}\right)\right)=\delta \hat{\boldsymbol{\xi}}\left(p\left({ }_{I} \boldsymbol{x}\right), q\left({ }_{I} \boldsymbol{x}\right), r\left({ }_{I} \boldsymbol{x}\right)\right)
$$

holds. Due to (45) and (47) together with the chain rule, we can reformulate the virtual work density in (44) now as

$$
\begin{aligned}
& \delta \boldsymbol{\xi}_{, x} \cdot \boldsymbol{t}_{x}+ \delta \boldsymbol{\xi}_{, y} \cdot \boldsymbol{t}_{y}+\delta \boldsymbol{\xi}_{, z} \cdot \boldsymbol{t}_{z} \\
&=\left(\delta \hat{\boldsymbol{\xi}}_{, p} \frac{\partial p}{\partial x}+\delta \hat{\boldsymbol{\xi}}_{, q} \frac{\partial q}{\partial x}+\delta \hat{\boldsymbol{\xi}}_{, r} \frac{\partial r}{\partial x}\right) \cdot \boldsymbol{t}_{x}+\left(\delta \hat{\boldsymbol{\xi}}_{, p} \frac{\partial p}{\partial y}+\delta \hat{\boldsymbol{\xi}}_{, q} \frac{\partial q}{\partial y}+\delta \hat{\boldsymbol{\xi}}_{, r} \frac{\partial r}{\partial y}\right) \cdot \boldsymbol{t}_{y}+\left(\delta \hat{\boldsymbol{\xi}}_{, p} \frac{\partial p}{\partial z}+\delta \hat{\boldsymbol{\xi}}_{, q} \frac{\partial q}{\partial z}+\delta \hat{\boldsymbol{\xi}}_{, r} \frac{\partial r}{\partial z}\right) \cdot \boldsymbol{t}_{z} \\
&=\delta \hat{\boldsymbol{\xi}}_{, p} \cdot\left(\frac{\partial p}{\partial x} \boldsymbol{t}_{x}+\frac{\partial p}{\partial y} \boldsymbol{t}_{y}+\frac{\partial p}{\partial z} \boldsymbol{t}_{z}\right)+\delta \hat{\boldsymbol{\xi}}_{, q} \cdot\left(\frac{\partial q}{\partial x} \boldsymbol{t}_{x}+\frac{\partial q}{\partial y} \boldsymbol{t}_{y}+\frac{\partial q}{\partial z} \boldsymbol{t}_{z}\right) \\
&+\delta \hat{\boldsymbol{\xi}}, r_{,} \cdot\left(\frac{\partial r}{\partial x} \boldsymbol{t}_{x}+\frac{\partial r}{\partial y} \boldsymbol{t}_{y}+\frac{\partial r}{\partial z} \boldsymbol{t}_{z}\right) \\
&=\delta \hat{\boldsymbol{\xi}}_{, p} \cdot \hat{\boldsymbol{t}}_{p}+\delta \hat{\boldsymbol{\xi}}_{, q} \cdot \hat{\boldsymbol{t}}_{q}+\delta \hat{\boldsymbol{\xi}}, r_{,} \cdot \hat{\boldsymbol{t}}_{r},
\end{aligned}
$$

where we have introduced the new stress vectors

$$
\begin{aligned}
& \hat{\boldsymbol{t}}_{p}=\frac{\partial p}{\partial x} \boldsymbol{t}_{x}+\frac{\partial p}{\partial y} \boldsymbol{t}_{y}+\frac{\partial p}{\partial z} \boldsymbol{t}_{z}, \\
& \hat{\boldsymbol{t}}_{q}=\frac{\partial q}{\partial x} \boldsymbol{t}_{x}+\frac{\partial q}{\partial y} \boldsymbol{t}_{y}+\frac{\partial q}{\partial z} \boldsymbol{t}_{z}, \\
& \hat{\boldsymbol{t}}_{r}=\frac{\partial r}{\partial x} \boldsymbol{t}_{x}+\frac{\partial r}{\partial y} \boldsymbol{t}_{y}+\frac{\partial r}{\partial z} \boldsymbol{t}_{z} .
\end{aligned}
$$


Using (48) together with (46) in (44), we observe the virtual work of the stress to be of the same form in cartesian and curvilinear coordinates:

$$
\begin{aligned}
\delta W^{\mathrm{s}}=-\int_{\Omega}\left(\delta \boldsymbol{\xi}_{, x} \cdot \boldsymbol{t}_{x}+\delta \boldsymbol{\xi}_{, y} \cdot \boldsymbol{t}_{y}\right. & \left.+\delta \boldsymbol{\xi}_{, z} \cdot \boldsymbol{t}_{z}\right) \mathrm{d} v \\
& =-\int_{\widehat{\Omega}}\left(\delta \hat{\boldsymbol{\xi}}_{, p} \cdot \hat{\boldsymbol{t}}_{p}+\delta \hat{\boldsymbol{\xi}}_{, q} \cdot \hat{\boldsymbol{t}}_{q}+\delta \hat{\boldsymbol{\xi}}_{, r} \cdot \hat{\boldsymbol{t}}_{r}\right) J \mathrm{~d} \hat{v}
\end{aligned}
$$

Note that the determinant of the Jacobian is $J=1$ for cartesian coordinates. Alternatively, we can consider $J \mathrm{~d} \hat{v}$ to be the Riemannian volume element, where $J$ corresponds to the square root of the first fundamental form of the mapping $\varphi$ [Kühnel 2013; Lee 2013]. Moreover, as a direct consequence of (50), it follows that the symmetry condition (40) applies in the same form when $(x, y, z)$ are curvilinear coordinates.

The integral in the rightmost expression of (44) is parametrized by the position vector $\boldsymbol{x}$ of the deformed configuration. Consequently, the domain of integration $\Omega$ changes for different configurations. It is often convenient to introduce a special configuration $\bar{\Omega}_{0}$, called the reference configuration, in which certain information, as for example the stress state and the dimensions of the body, are available. The position vectors $\boldsymbol{X}$ of the material points in the reference configuration are in a bijective relation with the position vectors $x$ in the deformed configuration; i.e., $\varphi: \bar{\Omega}_{0} \rightarrow \bar{\Omega}$ with $X \mapsto x=\varphi(X)$. Note, in contrast to (45), that the function $\varphi$ is here a mapping between subsets of $\mathbb{E}^{3}$ but not of $\mathbb{R}^{3}$. Using the mapping $\varphi$, we can express the integral in (44) with respect to the reference configuration $\bar{\Omega}_{0}=\varphi^{-1}(\bar{\Omega})$. This yields

$$
\mathrm{d} v=J \mathrm{~d} V \quad \text { with } J=\operatorname{det} \boldsymbol{F},
$$

where $\boldsymbol{F}=\partial \boldsymbol{\varphi} / \partial \boldsymbol{X}$ is the deformation gradient. The virtual displacement field over the reference configuration $\delta \boldsymbol{\Xi}: \bar{\Omega}_{0} \rightarrow \mathbb{E}^{3}$ is under the mapping $\varphi$ obtained as

$$
\delta \boldsymbol{\xi}(\boldsymbol{x})=\delta \boldsymbol{\Xi}\left(\boldsymbol{\varphi}^{-1}(\boldsymbol{x})\right) .
$$

Using (51) and (52) together with the chain rule, the last expression in (44) can be reformulated as

$$
\begin{aligned}
\delta W^{\mathrm{s}}=-\int_{\Omega} \frac{\partial \delta \boldsymbol{\xi}}{\partial \boldsymbol{x}}: \boldsymbol{\sigma} \mathrm{d} v=-\int_{\Omega_{0}}\left(\frac{\partial \delta \boldsymbol{\Xi}}{\partial \boldsymbol{X}} \cdot \frac{\partial \boldsymbol{\varphi}^{-1}}{\partial \boldsymbol{x}}\right): \boldsymbol{\sigma} J \mathrm{~d} V \\
=-\int_{\Omega_{0}}\left(\frac{\partial \delta \boldsymbol{\Xi}}{\partial \boldsymbol{X}} \cdot \boldsymbol{F}^{-1}\right): \boldsymbol{\sigma} J \mathrm{~d} V,
\end{aligned}
$$

where $\boldsymbol{F}^{-1}=\partial \boldsymbol{\varphi}^{-1} / \partial \boldsymbol{x}$ denotes the inverse of the deformation gradient. With the relation $(\boldsymbol{A} \cdot \boldsymbol{B}): \boldsymbol{C}=\boldsymbol{A}:\left(\boldsymbol{C} \cdot \boldsymbol{B}^{\mathrm{T}}\right)$, we rewrite (53) as

$$
\delta W^{\mathrm{s}}=-\int_{\Omega_{0}} \frac{\partial \delta \boldsymbol{\Xi}}{\partial \boldsymbol{X}}:\left(J \boldsymbol{\sigma} \cdot \boldsymbol{F}^{-\mathrm{T}}\right) \mathrm{d} V=-\int_{\Omega_{0}} \frac{\partial \delta \boldsymbol{\Xi}}{\partial \boldsymbol{X}}: \boldsymbol{P} \mathrm{d} V,
$$


in which the new stress representation, the first Piola-Kirchhoff stress,

$$
\boldsymbol{P}:=J \boldsymbol{\sigma} \cdot \boldsymbol{F}^{-\mathrm{T}}
$$

has been identified. Further manipulation of (53) leads to other stress representations [Bonet and Wood 1997]. The transformation of the virtual work expression guarantees the kinematical and the force quantities to always be work conjugate.

The last important transformation property is obtained when the stress vectors and the virtual displacement field are represented with respect to orthonormal frames. In addition to the $I$-system, we introduce the $B$-system $\left(\boldsymbol{e}_{x}^{B}, \boldsymbol{e}_{y}^{B}, \boldsymbol{e}_{z}^{B}\right)$ which is rotated with respect to the $I$-system. The position vector $\boldsymbol{x}$ represented in the $B$-system is given by the tuple ${ }_{B} \boldsymbol{x}=(\hat{x}, \hat{y}, \hat{z})^{\mathrm{T}}$. Since the two coordinate systems are rotated against each other, the coordinate representations of vectors $\boldsymbol{u} \in \mathbb{E}^{3}$ are related by

$$
{ }_{I} \boldsymbol{u}=\boldsymbol{A}_{I B B} \boldsymbol{u},
$$

with the transformation matrix $\boldsymbol{A}_{I B}$ being orthogonal, i.e., $\boldsymbol{A}_{I B}^{\mathrm{T}} \boldsymbol{A}_{I B}=\boldsymbol{A}_{I B} \boldsymbol{A}_{I B}^{\mathrm{T}}=\boldsymbol{I}$. The inverse of the transformation matrix $\boldsymbol{A}_{I B}$ is denoted as $\boldsymbol{A}_{B I}=\boldsymbol{A}_{I B}^{-1}=\boldsymbol{A}_{I B}^{\mathrm{T}}$. Furthermore, the coordinate representation of the virtual displacement field and its partial derivative in the $I$-system are

$$
\begin{aligned}
&{ }_{I} \delta \boldsymbol{\xi}\left({ }_{I} \boldsymbol{x}\right)=\left(\begin{array}{l}
\delta \xi_{x}^{I}\left({ }_{I} \boldsymbol{x}\right) \\
\delta \xi_{y}^{I}\left({ }_{I} \boldsymbol{x}\right) \\
\delta \xi_{z}^{I}\left({ }_{I} \boldsymbol{x}\right)
\end{array}\right), \\
& \frac{\partial_{I} \delta \boldsymbol{\xi}}{\partial_{I} \boldsymbol{x}} \equiv\left(\begin{array}{llll}
{ }_{I} \delta \boldsymbol{\xi}_{, x} & { }_{I} \delta \boldsymbol{\xi}_{, y} & { }_{I} \delta \boldsymbol{\xi}_{, z}
\end{array}\right)=\left(\begin{array}{lll}
\delta \xi_{x, x}^{I} & \delta \xi_{x, y}^{I} & \delta \xi_{x, z}^{I} \\
\delta \xi_{y, x}^{I} & \delta \xi_{y, y}^{I} & \delta \xi_{y, z}^{I} \\
\delta \xi_{z, x}^{I} & \delta \xi_{z, y}^{I} & \delta \xi_{z, z}^{I}
\end{array}\right) .
\end{aligned}
$$

By exchanging $(x, y, z)$ with $(\hat{x}, \hat{y}, \hat{z})$ and $I$ with $B$ in (57), we obtain the coordinate representation in the $B$-system. The same holds for the stress tensor $\sigma$, whose coordinate representation in the $I$-system is, due to (28), the matrix

$$
{ }_{I} \boldsymbol{\sigma}:=\left(\begin{array}{lll}
{ }_{I} \boldsymbol{t}_{x} & { }_{I} \boldsymbol{t}_{y}{ }_{I} \boldsymbol{t}_{z}
\end{array}\right)=\left(\begin{array}{ccc}
\sigma_{x x}^{I} & \tau_{x y}^{I} & \tau_{x z}^{I} \\
\tau_{y x}^{I} & \sigma_{y y}^{I} & \tau_{y z}^{I} \\
\tau_{z x}^{I} & \tau_{z y}^{I} & \sigma_{z z}^{I}
\end{array}\right) .
$$

The coordinate representation of the rightmost term in (44) is further transformed by using (56) and the chain rule to give

$$
\begin{array}{r}
\delta W^{\mathrm{s}=}=-\int_{\Omega} \frac{\partial_{I} \delta \xi}{\partial_{I} \boldsymbol{x}}:{ }_{I} \boldsymbol{\sigma} \mathrm{d} v=-\int_{\widehat{\Omega}}\left(\frac{\partial\left(\boldsymbol{A}_{I B B} \delta \boldsymbol{\xi}\right)}{\partial_{B} \boldsymbol{x}} \frac{\partial_{B} \boldsymbol{x}}{\partial_{I} \boldsymbol{x}}\right):{ }_{I} \boldsymbol{\sigma} \mathrm{d} \hat{v} \\
=-\int_{\widehat{\Omega}}\left(\boldsymbol{A}_{I B} \frac{\partial_{B} \delta \boldsymbol{\xi}}{\partial_{B} \boldsymbol{x}} \boldsymbol{A}_{I B}^{\mathrm{T}}\right):{ }_{I} \boldsymbol{\sigma} \mathrm{d} \hat{v}
\end{array}
$$


where $\widehat{\Omega}$ denotes the open set of the rotated domain with the volume element $\mathrm{d} \hat{v}=$ $\mathrm{d} \hat{x} \mathrm{~d} \hat{y} \mathrm{~d} \hat{z}$. Using the symmetry of the double contraction and twice the relation $(\boldsymbol{A} \boldsymbol{B}): \boldsymbol{C}=\boldsymbol{A}:\left(\boldsymbol{C} \boldsymbol{B}^{\mathrm{T}}\right)$, which holds also for matrices, (59) becomes

$$
\begin{aligned}
\delta W^{\mathrm{S}}=-\int_{\widehat{\Omega}} \frac{\partial_{B} \delta \xi}{\partial_{B} \boldsymbol{x}}:\left(\boldsymbol{A}_{I B}^{\mathrm{T}} \boldsymbol{\sigma} \boldsymbol{\sigma} \boldsymbol{A}_{I B}\right) \mathrm{d} \hat{v}=-\int_{\widehat{\Omega}} \frac{\partial_{B} \delta \xi}{\partial_{B} \boldsymbol{x}} & :\left(\boldsymbol{A}_{B I I} \boldsymbol{\sigma} \boldsymbol{A}_{B I}^{\mathrm{T}}\right) \mathrm{d} \hat{v} \\
& =-\int_{\widehat{\Omega}} \frac{\partial_{B} \delta \xi}{\partial_{B} \boldsymbol{x}}:{ }_{B} \boldsymbol{\sigma} \mathrm{d} \hat{v},
\end{aligned}
$$

with which the transformation rule of the coordinate representation of the stress tensor is identified to be

$$
{ }_{B} \sigma=A_{B I I} \sigma A_{B I}^{\mathrm{T}}
$$

The transformation rule (61) is of vital importance in the derivation of Mohr's circle, which is a graphical method to find the principal axis of the stress tensor $\sigma$.

\section{Boundary value problem}

Starting from the principle of virtual work (Axiom 1), we derive in this section the partial differential equations together with their boundary conditions, which describe a deformable body under the force interactions assumed as follows. As internal forces of the deformed configuration $\bar{\Omega}$, we allow solely stresses $\sigma$ that are short-range forces according to Definition 3. Any other kinds of internal forces, as for example force interactions of nonneighboring volume elements by long-range forces, are excluded. For the external forces, we assume that they either contribute as volume forces $f_{v} \mathrm{~d} v$ in the interior $\Omega$ of $\bar{\Omega}$, or as surface forces $f_{a} \mathrm{~d} a$ on the boundary $\partial \Omega$ of $\bar{\Omega}$. The following derivation corresponds de facto with the reverse direction of the approach presented by [Clebsch 1862; Lamé 1852].

To derive the boundary value problem, the virtual work of internal forces has to be integrated by parts, for which we use the following integral theorem of $\mathbb{R}^{3}$. Let $\Omega \subset \mathbb{R}^{3}$ be an open subset of $\mathbb{R}^{3}, \bar{\Omega}$ be the closure of $\Omega$, and $\partial \Omega=\bar{\Omega} \backslash \Omega$ be the boundary of $\Omega$. For a function $f(x, y, z)$ on $\Omega$, integration holds in the form

$$
\left.\int_{\Omega} \frac{\partial f}{\partial x} \mathrm{~d} v=\int_{\bar{\Omega}} \frac{\partial f}{\partial x} \mathrm{~d} v=\int_{\partial \Omega} f n^{x} \mathrm{~d} a \quad \text { (same for } y \text { and } z\right),
$$

where ${ }_{I} \boldsymbol{n}=\left(n^{x}, n^{y}, n^{z}\right)^{\mathrm{T}}$ are the coordinates of the outward unit normal $\boldsymbol{n}$ of $\Omega$.

In order to evaluate the principle of virtual work (5), the total virtual work $\delta W_{\text {tot }}$ of the body is required. The total virtual work is constituted of the virtual work contribution of the internal forces $\delta W^{\mathrm{i}}$ and the external forces $\delta W^{\mathrm{e}}$. Hence, the principle of virtual work (5) takes the form

$$
\delta W_{\text {tot }}=\delta W^{\mathrm{i}}+\delta W^{\mathrm{e}}=0 \quad \text { for all } \delta \xi .
$$


Since only stresses are allowed as internal forces, the internal forces contribute according to (44) as

$$
\delta W^{\mathrm{i}}=\delta W^{\mathrm{s}}=-\int_{\Omega} \frac{\partial \delta \boldsymbol{\xi}}{\partial \boldsymbol{x}}: \boldsymbol{\sigma} \mathrm{d} v=-\int_{\Omega}\left(\delta \boldsymbol{\xi}_{, x} \cdot \boldsymbol{t}_{x}+\delta \boldsymbol{\xi}_{, y} \cdot \boldsymbol{t}_{y}+\delta \boldsymbol{\xi}_{, z} \cdot \boldsymbol{t}_{z}\right) \mathrm{d} v .
$$

To avoid the divergence theorem for (second-order) tensors, we will use partial integration for the virtual work contribution $\delta W^{\mathrm{i}}$ in terms of the cartesian stress vectors $t_{i}$, as shown in the right part of (64). The application of this procedure to curvilinear coordinates is presented in the Appendix for the example of cylindrical coordinates. For now using the product rule, each of the three terms $\delta \boldsymbol{\xi}_{, i} \cdot \boldsymbol{t}_{i}$ in (64) is rewritten as

$$
\delta \boldsymbol{\xi}_{, x} \cdot \boldsymbol{t}_{x}=\left[\delta \boldsymbol{\xi} \cdot \boldsymbol{t}_{x}\right]_{, x}-\delta \boldsymbol{\xi} \cdot \boldsymbol{t}_{x, x} \quad(\text { same for } y \text { and } z),
$$

with which (64) becomes

$$
\delta W^{\mathrm{i}}=-\int_{\Omega}\left(\left[\delta \boldsymbol{\xi} \cdot \boldsymbol{t}_{x}\right]_{, x}+\left[\delta \boldsymbol{\xi} \cdot \boldsymbol{t}_{y}\right]_{, y}+\left[\delta \boldsymbol{\xi} \cdot \boldsymbol{t}_{z}\right]_{, z}\right) \mathrm{d} v+\int_{\Omega} \delta \boldsymbol{\xi} \cdot\left(\boldsymbol{t}_{x, x}+\boldsymbol{t}_{y, y}+\boldsymbol{t}_{z, z}\right) \mathrm{d} v .
$$

Applying now the divergence theorem (62) on each of the three terms $\left[\delta \boldsymbol{\xi} \cdot \boldsymbol{t}_{i}\right]_{, i}$ in the first integral, we obtain

$$
\begin{aligned}
\delta W^{\mathrm{i}}=-\int_{\partial \Omega}\left(\left[\delta \boldsymbol{\xi} \cdot \boldsymbol{t}_{x}\right] n^{x}+\left[\delta \boldsymbol{\xi} \cdot \boldsymbol{t}_{y}\right] n^{y}+\left[\delta \boldsymbol{\xi} \cdot \boldsymbol{t}_{z}\right] n^{z}\right) \mathrm{d} a & \\
& +\int_{\Omega} \delta \boldsymbol{\xi} \cdot\left(\boldsymbol{t}_{x, x}+\boldsymbol{t}_{y, y}+\boldsymbol{t}_{z, z}\right) \mathrm{d} v .
\end{aligned}
$$

With $\boldsymbol{n}=n^{x} \boldsymbol{e}_{x}^{I}+n^{y} \boldsymbol{e}_{y}^{I}+n^{z} \boldsymbol{e}_{z}^{I}$ and the definition of the stress tensor $\boldsymbol{\sigma}$ according to (28), the virtual work contribution (67) may now be rewritten as

$$
\delta W^{\mathrm{i}}=-\int_{\partial \Omega} \delta \boldsymbol{\xi} \cdot \boldsymbol{\sigma} \cdot \boldsymbol{n} \mathrm{d} a+\int_{\Omega} \delta \boldsymbol{\xi} \cdot\left(\boldsymbol{t}_{x, x}+\boldsymbol{t}_{y, y}+\boldsymbol{t}_{z, z}\right) \mathrm{d} v,
$$

which is the desired strong variational form. By following our assumption about the external loading of the body, only volume forces $f_{v}$ and surface forces $f_{a}$ are considered. The external virtual work is therefore given by (11) as

$$
\delta W^{\mathrm{e}}=\int_{\Omega} \delta \boldsymbol{\xi} \cdot \boldsymbol{f}_{v} \mathrm{~d} v+\int_{\partial \Omega} \delta \boldsymbol{\xi} \cdot \boldsymbol{f}_{a} \mathrm{~d} a .
$$

The total virtual work (63) is obtained by summing up (68) and (69), which gives the strong variational form of the principle of virtual work in the deformed configuration $\bar{\Omega}$ :

$\delta W_{\mathrm{tot}}=\int_{\Omega} \delta \boldsymbol{\xi} \cdot\left(\boldsymbol{t}_{x, x}+\boldsymbol{t}_{y, y}+\boldsymbol{t}_{z, z}+\boldsymbol{f}_{v}\right) \mathrm{d} v+\int_{\partial \Omega} \delta \boldsymbol{\xi} \cdot\left(\boldsymbol{f}_{a}-\boldsymbol{\sigma} \cdot \boldsymbol{n}\right) \mathrm{d} a=0$ 
We now apply again the fundamental lemma of calculus of variations under the following choices of variations: in a first step, we choose virtual displacement fields with $\delta \xi=0$ on the boundary $\partial \Omega$ and $\delta \xi$ arbitrary in the interior $\Omega$, and in a second step, we let $\delta \xi$ be arbitrary on the boundary $\partial \Omega$. This results in the two localized conditions

$$
\begin{aligned}
& \boldsymbol{t}_{x, x}+\boldsymbol{t}_{y, y}+\boldsymbol{t}_{z, z}=-\boldsymbol{f}_{v} \quad \text { in } \Omega \quad \text { (local equilibrium conditions), } \\
& \boldsymbol{\sigma} \cdot \boldsymbol{n}=\boldsymbol{f}_{a} \quad \text { on } \partial \Omega \quad \text { (boundary conditions), }
\end{aligned}
$$

which are the partial differential equations in the interior $\Omega$ and the boundary conditions for the forces on $\partial \Omega$, forming together the classical boundary value problem of continuum mechanics.

\section{Integral balance laws}

So far, we have not yet drawn any conclusions about how the subsystems of $\bar{\Omega}$ interact with each other. The answer to this question will be given at the end of this section in the inverse stress theorem, of which the argumentation follows the opposite direction as in Cauchy's stress theorem. The interaction between the subsystems can only take place by their external forces, since their internal forces, by definition, cannot interact with their environments. Hence, we need to determine the external forces for an arbitrary subsystem $\bar{K}$ of the continuum. For this, the total virtual work of $\bar{\Omega}$ is required and has to be evaluated for those virtual displacement fields that correspond with rigid virtual displacements on the considered subsystem $\bar{K}$ and are zero elsewhere. By doing so, the internal forces of $\bar{K}$ drop out, as do any other forces not acting on the points of $\bar{K}$, and only the external forces of $\bar{K}$ remain. This special choice of virtual displacement fields is neither smooth nor continuous. But as has already been recognized by [Hellinger 1914, §3.d], which is commented on in [Eugster and dell'Isola 2017a], one succeeds with approximating such fields by a family of continuous virtual displacements. Strichartz [1994, §6.6] shows that such an approximation can even be achieved with smooth test functions, i.e., with smooth virtual displacement fields.

For the sake of brevity and without loss of generality, we choose $\bar{K}$ to be an inner subsystem. An inner subsystem $\bar{K}$ of $\bar{\Omega}$ is understood in this context to be a closed subset of $\bar{\Omega}$, whose boundary $\partial K$ does not have any material points in common with the boundary $\partial \Omega$ of $\bar{\Omega}$. As depicted in Figure 5 , we denote the boundary of $\bar{K}$ by $\partial K$, the interior of $\bar{K}$ by $K$, and the complement of $\bar{K}$ with respect to $\bar{\Omega}$ as $H:=\bar{\Omega} \backslash \bar{K}$. Hence, $\bar{\Omega}$ can be subdivided into the disjoint sets

$$
\bar{\Omega}=K \cup \partial K \cup H=\bar{K} \cup H \quad \text { with } \bar{K}=K \cup \partial K \text {. }
$$



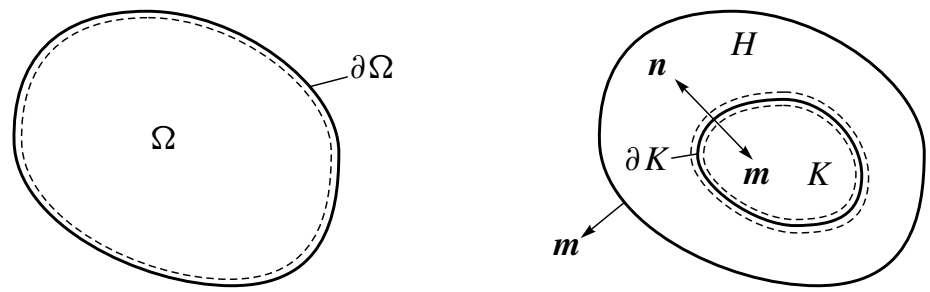

Figure 5. Disjoint subdivision of the deformed configuration $\bar{\Omega}$ into an inner subsystem $\bar{K}$ and its complement $H=\bar{\Omega} \backslash \bar{K}$. The outward normals of $K$ and $H$ are denoted by $\boldsymbol{n}$ and $\boldsymbol{m}$, respectively. The common boundary of $K$ and $H$ is given by the boundary of $K$, denoted as $\partial K$.

For the boundaries, we have

$$
\partial H=\partial \Omega \cup \partial K \quad \text { with } \boldsymbol{m}=-\boldsymbol{n} \text { on } \partial K,
$$

where $\boldsymbol{n}$ and $\boldsymbol{m}$ are the outward-pointing unit normals of $K$ and $H$, respectively.

For the formulation of the total virtual work $\delta W_{\text {tot }}$ of $\bar{\Omega}$ according to (70), we sum up the contributions of the total virtual work $\delta W_{\text {tot }}^{H}$ and $\delta W_{\text {tot }}^{K}$ of $H$ and $K$, respectively. For $\delta W_{\text {tot }}^{H}$, we obtain similarly to (70)

$$
\begin{array}{r}
\delta W_{\mathrm{tot}}^{H}=\int_{H} \delta \boldsymbol{\xi} \cdot\left(\boldsymbol{t}_{x, x}+\boldsymbol{t}_{y, y}+\boldsymbol{t}_{z, z}+\boldsymbol{f}_{v}\right) \mathrm{d} v+\int_{\partial \Omega} \delta \boldsymbol{\xi} \cdot\left(\boldsymbol{f}_{a}-\boldsymbol{\sigma} \cdot \boldsymbol{m}\right) \mathrm{d} a \\
-\int_{\partial K} \delta \boldsymbol{\xi} \cdot \boldsymbol{\sigma} \cdot \boldsymbol{m} \mathrm{d} a,
\end{array}
$$

where, according to our loading assumptions, the external surface forces $f_{a}$ of $\bar{\Omega}$ act only on the subset $\partial \Omega$ of $\partial H$, but not on the boundary $\partial K$ that was generated by the subdivision (72) and (73). Consequently, the total virtual work $\delta W_{\text {tot }}^{K}$ of $K$ becomes

$$
\delta W_{\mathrm{tot}}^{K}=\int_{K} \delta \boldsymbol{\xi} \cdot\left(\boldsymbol{t}_{x, x}+\boldsymbol{t}_{y, y}+\boldsymbol{t}_{z, z}+\boldsymbol{f}_{v}\right) \mathrm{d} v-\int_{\partial K} \delta \boldsymbol{\xi} \cdot \boldsymbol{\sigma} \cdot \boldsymbol{n} \mathrm{d} a .
$$

By adding (74) and (75), one obtains

$$
\delta W_{\mathrm{tot}}^{H}+\delta W_{\mathrm{tot}}^{K}=\delta W_{\mathrm{tot}},
$$

which is, due to $\boldsymbol{m}=-\boldsymbol{n}$ on $\partial K$, again the virtual work according to (70).

To extract now from the total virtual work (76) only the external virtual work contribution of $\bar{K}$, we choose for $\delta \xi$ the rigid virtual displacements on $\bar{K}$, which implies

$$
\delta W_{\text {tot }}=0 \quad \text { for all } \delta \boldsymbol{\xi}_{\bar{K}, \text { rig }}(\boldsymbol{x})= \begin{cases}\delta \boldsymbol{\xi}_{\text {rig }}(\boldsymbol{x}) & \text { for } \boldsymbol{x} \in \bar{K}=K \cup \partial K, \\ 0 & \text { for } \boldsymbol{x} \in H .\end{cases}
$$


With $\delta \boldsymbol{\xi}_{\bar{K}, \text { rig }}(\boldsymbol{x})=0$ for $\boldsymbol{x} \in H$, the virtual work contributions

$$
\begin{array}{r}
\int_{H} \delta \boldsymbol{\xi}_{\bar{K}, \text { rig }} \cdot\left(\boldsymbol{t}_{x, x}+\boldsymbol{t}_{y, y}+\boldsymbol{t}_{z, z}+\boldsymbol{f}_{v}\right) \mathrm{d} v=0, \\
\int_{\partial \Omega} \delta \boldsymbol{\xi}_{\bar{K}, \text { rig }} \cdot\left(\boldsymbol{f}_{a}-\boldsymbol{\sigma} \cdot \boldsymbol{m}\right) \mathrm{d} a=0
\end{array}
$$

vanish from $\delta W_{\text {tot }}^{H}$ in (74), and the variational problem (76) and (77) reduces with the remaining contributions from (74) and (75) to

$\delta W_{\mathrm{tot}}=\int_{K} \delta \boldsymbol{\xi}_{\mathrm{rig}} \cdot\left(\boldsymbol{t}_{x, x}+\boldsymbol{t}_{y, y}+\boldsymbol{t}_{z, z}+\boldsymbol{f}_{v}\right) \mathrm{d} v-\int_{\partial K} \delta \boldsymbol{\xi}_{\mathrm{rig}} \cdot(\boldsymbol{\sigma} \cdot \boldsymbol{n}+\boldsymbol{\sigma} \cdot \boldsymbol{m}) \mathrm{d} a=0$
for all $\delta \boldsymbol{\xi}_{\mathrm{rig}}$.

The contribution of the internal forces of $K$, consisting in our case of just the stresses in $K$, cancels out of (79) because of (41). To demonstrate this explicitly, we reverse the partial integration (64)-(68), which gives

$$
\int_{K} \delta \boldsymbol{\xi}_{\mathrm{rig}} \cdot\left(\boldsymbol{t}_{x, x}+\boldsymbol{t}_{y, y}+\boldsymbol{t}_{z, z}\right) \mathrm{d} v-\int_{\partial K} \delta \boldsymbol{\xi}_{\mathrm{rig}} \cdot \boldsymbol{\sigma} \cdot \boldsymbol{n} \mathrm{d} a=-\int_{K} \frac{\partial \delta \boldsymbol{\xi}_{\mathrm{rig}}}{\partial \boldsymbol{x}}: \boldsymbol{\sigma} \mathrm{d} v=0
$$

According to this, (79) reduces to

$$
\delta W_{\mathrm{tot}}=\int_{K} \delta \boldsymbol{\xi}_{\mathrm{rig}} \cdot \boldsymbol{f}_{v} \mathrm{~d} v-\int_{\partial K} \delta \boldsymbol{\xi}_{\mathrm{rig}} \cdot \boldsymbol{\sigma} \cdot \boldsymbol{m} \mathrm{d} a=0 \quad \text { for all } \delta \boldsymbol{\xi}_{\mathrm{rig}} .
$$

In addition, the identity $\boldsymbol{m}=-\boldsymbol{n}$ holds on $\partial K$ by (73) for the outward normal $\boldsymbol{m}$ of $H$. Furthermore, by setting

$$
t:=\sigma \cdot n
$$

we can reformulate (81) as

$$
0=\int_{K} \delta \xi_{\text {rig }} \cdot \boldsymbol{f}_{v} \mathrm{~d} v+\int_{\partial K} \delta \boldsymbol{\xi}_{\mathrm{rig}} \cdot \boldsymbol{t} \mathrm{d} a \quad \text { for all } \delta \boldsymbol{\xi}_{\text {rig }} .
$$

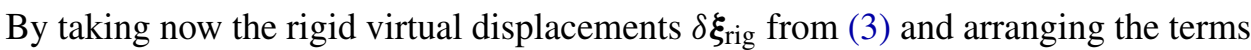
with respect to $\delta \boldsymbol{r}_{O}$ and $\delta \boldsymbol{\phi}$, one obtains

$$
\begin{aligned}
& 0=\int_{K}\left(\delta \boldsymbol{r}_{O}+\delta \boldsymbol{\phi} \times \boldsymbol{x}\right) \cdot \boldsymbol{f}_{v} \mathrm{~d} v+\int_{\partial K}\left(\delta \boldsymbol{r}_{O}+\delta \boldsymbol{\phi} \times \boldsymbol{x}\right) \cdot \boldsymbol{t} \mathrm{d} a \\
&=\delta \boldsymbol{r}_{O} \cdot\left(\int_{K} \boldsymbol{f}_{v} \mathrm{~d} v+\int_{\partial K} \boldsymbol{t} \mathrm{d} a\right)+\delta \boldsymbol{\phi} \cdot\left(\int_{K} \boldsymbol{x} \times \boldsymbol{f}_{V} \mathrm{~d} v+\int_{\partial K} \boldsymbol{x} \times \boldsymbol{t} \mathrm{d} a\right) \\
& \text { for all } \delta \boldsymbol{r}_{O} \text { and all } \delta \boldsymbol{\phi} .
\end{aligned}
$$

After the evaluation of the two independent variations for $\delta \boldsymbol{r}_{O}$ and $\delta \boldsymbol{\phi}$, one ends up 
with the integral balance laws for the external forces and moments acting on $\bar{K}$ :

$$
\begin{aligned}
\int_{K} \boldsymbol{f}_{v} \mathrm{~d} v+\int_{\partial K} \boldsymbol{t} \mathrm{d} a & =0 \quad \text { (equilibrium of external forces), } \\
\int_{K} \boldsymbol{x} \times \boldsymbol{f}_{v} \mathrm{~d} v+\int_{\partial K} \boldsymbol{x} \times \boldsymbol{t} \mathrm{d} a & =0 \quad \text { (equilibrium of external moments). }
\end{aligned}
$$

With $\boldsymbol{t}=\boldsymbol{\sigma} \cdot \boldsymbol{n}$ from (82) and $\boldsymbol{m}=-\boldsymbol{n}$ from (73), it is now also apparent, how the subsystems of $\bar{\Omega}$ interact with each other:

Theorem 1 (inverse stress theorem). Let the internal forces of $\bar{\Omega}$ consist only of the continuous stress tensor field $\sigma$, and let $\bar{K}$ be an (inner) subsystem of $\bar{\Omega}$. Then the complement $H=\bar{\Omega} \backslash \bar{K}$ of $\bar{K}$ exerts on the (inner) subsystem $\bar{K}$ the force and the moment

$$
\int_{\partial K} \boldsymbol{t} \mathrm{d} a \text { and } \int_{\partial K} \boldsymbol{x} \times \boldsymbol{t} \mathrm{d} a
$$

via the surface $\partial K$ of $\bar{K}$, where $t$ depends linearly on the outward-pointing unit normal $\boldsymbol{n}$ of $\bar{K}$ by $\boldsymbol{t}=\boldsymbol{\sigma} \cdot \boldsymbol{n}$.

In the nonvariational approach to mechanics, the stress principle of Euler and Cauchy assumes the interaction between subsystems to take place by surface forces only. Cauchy's stress theorem then shows that these surface forces depend linearly on the normal of the contact surface, which asserts the existence of the stress tensor field. Since we proposed in this paper an alternative notion of stress, the stress tensor field follows already from the derivation in Section 4, but the interaction mechanism between the subsystems remains unexplained. This interaction mechanism is now formulated in Theorem 1, called the inverse stress theorem, as it demonstrates the reverse direction of Cauchy's stress theorem.

\section{Conclusion}

In this paper, the controversy about the a priori notion of the contribution of the internal virtual work in a variational formulation has been resolved by an alternative perspective on the notion of stress. This notion relies on the interpretation of Piola's micro-macro identification procedure in view of the Riemann integral, which naturally provides in its mathematical definition a micro-macro relation between the discrete system of infinitesimal volume elements and the continuum. Accordingly, we proposed a definition of stress in the sense of Boltzmann on the micro level of the infinitesimal volume elements. In particular, when the stress is defined as the internal force effects of the body that model the mutual force interaction between neighboring infinitesimal volume elements, Piola's micro-macro identification procedure leads directly to the internal virtual work of the classical continuum. In the course of this, the stress tensor emerges as the quantity dual to 
the gradient of the virtual displacement field. It seems that Piola's micro-macro identification procedure gives us a very intuitive tool to model a wide variety of force interactions which can appear in a continuum.

In contrast to the nonvariational formulation, in which the stress principle of Euler and Cauchy defines the concept of stress, no assumption has been made about the mechanism of interaction between subsystems, in order to show the existence of the stress tensor. Within the variational formulation, the mechanism of interaction between the subsystems follows from the inverse stress theorem, which effectively shows the reverse direction of Cauchy's stress theorem. The derivation of the virtual work contribution of the stress as given in Section 4, together with the inverse stress theorem, can be considered as the variational counterpart to Cauchy's stress theorem. In the variational formulation, the symmetry of the stress tensor is a direct consequence of the variational law of interaction and the continuity of the stress field. Since the virtual work is by definition an invariant expression, the virtual work provides for different integral parametrizations or coordinate representations the required transformation properties.

As variational methods are inevitable for generalized continua, i.e., continua with microstructure or higher-order continua, it needs to be shown how the introduced notion of stress can be applied to those theories. In the present approach, neighboring volume elements have been understood as adjacent volume elements sharing the same surface. However, Piola's nonlocal theory makes us confident to conjecture that the consideration of additional interactions between volume elements sharing the same edges and wedges, or which are even nonadjacent, will lead to higher-order stresses as known from $N$-th gradient theories. Nevertheless, the proposed definition of stress on the micro level of infinitesimal volume elements would still hold in its essence.

In order to focus on the concept of stress, we have omitted the discussion about inertia forces and inertial frames. By adding the inertia forces in the sense of d'Alembert as external volume forces to the total virtual work, the equations of motion in variational form and consequently a theory of dynamics is obtained. Such a variational formulation already forms the basis for many approaches in analytical dynamics, as for example the Lagrangian equations of motion, Hamilton's principle, or the projected Newton-Euler equations. As a consequence, both continuum mechanics and analytical dynamics are based on the very same axioms. The theories differ merely in the modeling of the corresponding force interactions.

\section{Appendix: Equilibrium equations in cylindrical coordinates}

In order to avoid the concept of co- and contravariant basis vectors, (50) is a convenient starting point to derive the local equilibrium conditions also for curvilinear 
coordinates. As an example, we choose cylindrical coordinates $(p, q, r)=(r, \varphi, z)$ which are related to the cartesian coordinates by

$$
{ }_{I} \boldsymbol{x}=\boldsymbol{\varphi}(r, \varphi, z)=(r \cos \varphi, r \sin \varphi, z)^{\mathrm{T}},
$$

with the determinant of the Jacobian $J=\operatorname{det}(D \varphi)(r, \varphi, z)=r$. The virtual work of internal forces according to (50) is

$$
\delta W^{\mathrm{i}}=-\int_{\Omega}\left(\delta \boldsymbol{\xi}_{, r} \cdot \boldsymbol{t}_{r}+\delta \boldsymbol{\xi}_{, \varphi} \cdot \boldsymbol{t}_{\varphi}+\delta \boldsymbol{\xi}_{, z} \cdot \boldsymbol{t}_{z}\right) r \mathrm{~d} v,
$$

where we have omitted the hat and have used the volume element (46). With the volume element $r \mathrm{~d} v=r \mathrm{~d} r \mathrm{~d} \varphi \mathrm{d} z$, the virtual work densities within the integral have the units $\left[\delta \boldsymbol{\xi}_{, i} \cdot \boldsymbol{t}_{i}\right]=\mathrm{Nm} / \mathrm{m}^{3}$. In contrast to $\boldsymbol{t}_{r}$ and $\boldsymbol{t}_{z}$ having units $\left[\boldsymbol{t}_{i}\right]=\mathrm{N} / \mathrm{m}^{2}$, the stress vector $\boldsymbol{t}_{\varphi}$ has, due to the dimensionless partial derivative $\delta \boldsymbol{\xi}_{, \varphi}$, the unit $\left[\boldsymbol{t}_{\varphi}\right]=\mathrm{N} / \mathrm{m}^{3}$. This dimensional mismatch can be corrected by introducing the socalled physical stress vectors $\boldsymbol{\sigma}_{r}, \boldsymbol{\sigma}_{\varphi}, \boldsymbol{\sigma}_{z}$ [Başar and Weichert 2000, p. 101], defined by the relations

$$
\boldsymbol{t}_{r}=\boldsymbol{\sigma}_{r}, \quad \boldsymbol{t}_{\varphi}=\frac{1}{r} \boldsymbol{\sigma}_{\varphi}, \quad \boldsymbol{t}_{z}=\boldsymbol{\sigma}_{z} .
$$

The virtual work (88) together with the physical stress vectors (89) leads to ${ }^{16}$

$$
\delta W^{\mathrm{i}}=-\int_{\Omega}\left(\delta \boldsymbol{\xi}_{, r} \cdot \boldsymbol{\sigma}_{r}+\delta \boldsymbol{\xi}_{, \varphi} \cdot \frac{1}{r} \boldsymbol{\sigma}_{\varphi}+\delta \boldsymbol{\xi}_{, z} \cdot \boldsymbol{\sigma}_{z}\right) r \mathrm{~d} v .
$$

By using the product rule, we can reformulate each of the terms in (90) according to

$$
\begin{aligned}
\delta \boldsymbol{\xi}_{, r} \cdot \boldsymbol{\sigma}_{r} r & =\left[\delta \boldsymbol{\xi} \cdot \boldsymbol{\sigma}_{r} r\right]_{, r}-\delta \boldsymbol{\xi} \cdot\left[\boldsymbol{\sigma}_{r, r}+\frac{1}{r} \boldsymbol{\sigma}_{r}\right] r, \\
\delta \boldsymbol{\xi}_{, \varphi} \cdot \boldsymbol{\sigma}_{\varphi} & =\left[\delta \boldsymbol{\xi} \cdot \boldsymbol{\sigma}_{\varphi}\right]_{, \varphi}-\delta \boldsymbol{\xi} \cdot\left[\frac{1}{r} \boldsymbol{\sigma}_{\varphi, \varphi}\right] r, \\
\delta \boldsymbol{\xi}_{, z} \cdot \boldsymbol{\sigma}_{z} r & =\left[\delta \boldsymbol{\xi} \cdot \boldsymbol{\sigma}_{z} r\right]_{, z}-\delta \boldsymbol{\xi} \cdot \boldsymbol{\sigma}_{z, z} r .
\end{aligned}
$$

Applying the identities (91) to (88), we reformulate the internal virtual work as

$$
\begin{aligned}
\delta W^{\mathrm{i}}=-\int_{\Omega}\left(\left[\delta \boldsymbol{\xi} \cdot \boldsymbol{\sigma}_{r} r\right]_{, r}+\right. & {\left.\left[\delta \boldsymbol{\xi} \cdot \boldsymbol{\sigma}_{\varphi}\right]_{, \varphi}+\left[\delta \boldsymbol{\xi} \cdot \boldsymbol{\sigma}_{z} r\right]_{, z}\right) \mathrm{d} r \mathrm{~d} \varphi \mathrm{d} z } \\
& +\int_{\Omega} \delta \boldsymbol{\xi} \cdot\left(\boldsymbol{\sigma}_{r, r}+\frac{1}{r} \boldsymbol{\sigma}_{r}+\frac{1}{r} \boldsymbol{\sigma}_{\varphi, \varphi}+\boldsymbol{\sigma}_{z, z}\right) r \mathrm{~d} r \mathrm{~d} \varphi \mathrm{d} z .
\end{aligned}
$$

After carrying out the integration (62) of the first term in (92), the virtual displacements are chosen such that $\delta \xi=0$ on the boundary $\partial \Omega$, which yields

$$
\delta W^{\mathrm{i}}=\int_{\Omega} \delta \xi \cdot\left(\sigma_{r, r}+\frac{1}{r} \sigma_{r}+\frac{1}{r} \sigma_{\varphi, \varphi}+\sigma_{z, z}\right) r \mathrm{~d} r \mathrm{~d} \varphi \mathrm{d} z .
$$

${ }^{16}$ Alternatively, (90) can be obtained by evaluating the expression $\partial \delta \boldsymbol{\xi} / \partial \boldsymbol{x}: \boldsymbol{\sigma}=\left(\delta \boldsymbol{\xi}_{, r} \otimes \partial r / \partial \boldsymbol{x}+\right.$ $\left.\delta \boldsymbol{\xi}_{, \varphi} \otimes \partial \varphi / \partial \boldsymbol{x}+\delta \boldsymbol{\xi}_{, z} \otimes \partial z / \partial \boldsymbol{x}\right):\left(\boldsymbol{\sigma}_{r} \otimes \boldsymbol{e}_{r}+\boldsymbol{\sigma}_{\varphi} \otimes \boldsymbol{e}_{\varphi}+\boldsymbol{\sigma}_{z} \otimes \boldsymbol{e}_{z}\right)$ using the basis vectors of (97). 
As in Section 8, the external forces are assumed to be either volume forces or surface forces on the boundary of the body. Consequently, just the volume forces $\boldsymbol{f}_{v}$ contribute to the virtual work,

$$
\delta W^{\mathrm{e}}=\int_{\Omega} \delta \xi \cdot f_{v} r \mathrm{~d} r \mathrm{~d} \varphi \mathrm{d} z
$$

as the virtual displacements have been chosen to vanish on the boundary. With $\delta W_{\text {tot }}=\delta W^{\mathrm{i}}+\delta W^{\mathrm{e}}$ together with the contributions (93) and (94), the principle of virtual work (5) implies that

$$
\delta W_{\mathrm{tot}}=\int_{\Omega} \delta \xi \cdot\left(\boldsymbol{\sigma}_{r, r}+\frac{1}{r} \boldsymbol{\sigma}_{r}+\frac{1}{r} \boldsymbol{\sigma}_{\varphi, \varphi}+\boldsymbol{\sigma}_{z, z}+\boldsymbol{f}_{v}\right) r \mathrm{~d} r \mathrm{~d} \varphi \mathrm{d} z=0 \quad \text { for all } \delta \xi
$$

By the fundamental lemma of calculus of variations, the strong variational form (95) leads directly to the local equilibrium conditions

$$
\boldsymbol{\sigma}_{r, r}+\frac{1}{r} \sigma_{r}+\frac{1}{r} \sigma_{\varphi, \varphi}+\sigma_{z, z}+f_{v}=0
$$

It is convenient to represent the physical stress vectors in the cylindrical orthonormal basis $\left(\boldsymbol{e}_{r}, \boldsymbol{e}_{\varphi}, \boldsymbol{e}_{z}\right)$, which depends on its position $(r, \varphi, z)$ and is defined with respect to the $I$-system as

$$
{ }_{I} \boldsymbol{e}_{r}=\left(\begin{array}{c}
\cos \varphi \\
\sin \varphi \\
0
\end{array}\right), \quad{ }_{I} \boldsymbol{e}_{\varphi}=\left(\begin{array}{c}
-\sin \varphi \\
\cos \varphi \\
0
\end{array}\right), \quad{ }_{I} \boldsymbol{e}_{z}=\left(\begin{array}{l}
0 \\
0 \\
1
\end{array}\right)
$$

By computing the partial derivative of the basis vectors with respect to $(r, \varphi, z)$, it can easily be shown that the only nonvanishing contributions are

$$
\boldsymbol{e}_{r, \varphi}=\boldsymbol{e}_{\varphi}, \quad \boldsymbol{e}_{\varphi, \varphi}=-\boldsymbol{e}_{r}
$$

For an explicit evaluation of (96) in components, the following computations are of importance:

$$
\begin{aligned}
& \boldsymbol{\sigma}_{r, r}=\left(\sigma_{r r} \boldsymbol{e}_{r}+\tau_{\varphi r} \boldsymbol{e}_{\varphi}+\tau_{z r} \boldsymbol{e}_{z}\right)_{, r}=\left(\sigma_{r r, r} \boldsymbol{e}_{r}+\tau_{\varphi r, r} \boldsymbol{e}_{\varphi}+\tau_{z r, r} \boldsymbol{e}_{z}\right), \\
& \boldsymbol{\sigma}_{\varphi, \varphi}=\left(\tau_{r \varphi} \boldsymbol{e}_{r}+\sigma_{\varphi \varphi} \boldsymbol{e}_{\varphi}+\tau_{z \varphi} \boldsymbol{e}_{z}\right)_{, \varphi}=\left(\tau_{r \varphi, \varphi} \boldsymbol{e}_{r}+\tau_{r \varphi} \boldsymbol{e}_{\varphi}+\sigma_{\varphi \varphi, \varphi} \boldsymbol{e}_{\varphi}-\sigma_{\varphi \varphi} \boldsymbol{e}_{r}+\tau_{z \varphi, \varphi} \boldsymbol{e}_{z}\right), \\
& \boldsymbol{\sigma}_{z, z}=\left(\tau_{r z} \boldsymbol{e}_{r}+\tau_{\varphi z} \boldsymbol{e}_{\varphi}+\boldsymbol{\sigma}_{z z} \boldsymbol{e}_{z}\right)_{, z}=\tau_{r z, r} \boldsymbol{e}_{r}+\tau_{\varphi z, z} \boldsymbol{e}_{\varphi}+\sigma_{z z, z} \boldsymbol{e}_{z}, \\
& \boldsymbol{f}_{v}=f_{r} \boldsymbol{e}_{r}+f_{\varphi} \boldsymbol{e}_{\varphi}+f_{z} \boldsymbol{e}_{z} .
\end{aligned}
$$


Using (99) within (96), we end up with the local equilibrium equations described in the components of the $\left(\boldsymbol{e}_{r}, \boldsymbol{e}_{\varphi}, \boldsymbol{e}_{z}\right)$-system:

$$
\begin{aligned}
& 0=\sigma_{r r, r}+\frac{1}{r} \sigma_{r r}+\frac{1}{r} \tau_{r \varphi, \varphi}-\frac{1}{r} \sigma_{\varphi \varphi}+\tau_{r z, z}+f_{r}, \\
& 0=\tau_{\varphi r, r}+\frac{1}{r} \tau_{\varphi r}+\frac{1}{r} \sigma_{\varphi \varphi, \varphi}+\frac{1}{r} \tau_{r \varphi}+\tau_{\varphi z, z}+f_{\varphi}, \\
& 0=\tau_{z r, r}+\frac{1}{r} \tau_{z r}+\frac{1}{r} \tau_{z \varphi, \varphi}+\sigma_{z z, z}+f_{z},
\end{aligned}
$$

which is often derived by geometrical arguments on the cylindrical volume elements [Timoshenko and Goodier 1951, p. 55]. The same procedure as proposed here can also be applied to obtain the Lagrangian equilibrium equation in cylindrical and spherical coordinates, which are presented in [Volokh 2006].

\section{References}

[Altenbach and Altenbach 1994] J. Altenbach and H. Altenbach, Einführung in die Kontinuumsmechanik, Teubner, 1994.

[Başar and Weichert 2000] Y. Başar and D. Weichert, Nonlinear continuum mechanics of solids: fundamental mathematical and physical concepts, Springer, 2000.

[Becker and Bürger 1975] E. Becker and W. Bürger, Kontinuumsmechanik: eine Einführung in die Grundlagen und einfache Anwendungen, Leitfäden der Angewandten Mathematik und Mechanik 20, Teubner, 1975.

[Bertram 1989] A. Bertram, Axiomatische Einführung in die Kontinuumsmechanik, Bibliographisches Institut, 1989.

[Bertram 2012] A. Bertram, Elasticity and plasticity of large deformations: an introduction, 3rd ed., Springer, 2012.

[Bertram and Forest 2007] A. Bertram and S. Forest, "Mechanics based on an objective power functional", Tech. Mech. 27:1 (2007), 1-17.

[Boltzmann 1905] L. Boltzmann, Populäre Schriften, Barth, 1905.

[Bonet and Wood 1997] J. Bonet and R. D. Wood, Nonlinear continuum mechanics for finite element analysis, Cambridge University, 1997.

[Capecchi and Ruta 2015] D. Capecchi and G. Ruta, Strength of materials and theory of elasticity in 19th century Italy: a brief account of the history of mechanics of solids and structures, Advanced Structured Materials 52, Springer, 2015.

[Cauchy 1823] A. L. Cauchy, "Recherches sur l'équilibre et le mouvement intérieur des corps solides ou fluides, élastiques ou non élastiques", pp. 300-304 in Euvres complètes, II, tome II, Gauthier-Villars, 1823.

[Cauchy 1827a] A. L. Cauchy, "De la pression ou tension dans un corps solide", pp. 60-78 in CEuvres complètes, II, tome VII, Gauthier-Villars, 1827.

[Cauchy 1827b] A. L. Cauchy, "Sur les relations qui existent dans l'état d'équilibre d'un corps solide ou fluide, entre les pressions ou tensions et les forces accélératrices", pp. 141-145 in Euvres complètes, II, tome VII, Gauthier-Villars, 1827.

[Chadwick 1999] P. Chadwick, Continuum mechanics: concise theory and problems, 2nd ed., Dover, 1999. 
[Ciarlet 1988] P. G. Ciarlet, Mathematical elasticity, vol. I: Three-dimensional elasticity, Studies in Mathematics and its Applications 20, North-Holland, 1988.

[Clebsch 1862] A. Clebsch, Theorie der Elasticität fester Körper, Teubner, 1862.

[Del Piero 2009] G. Del Piero, "On the method of virtual power in continuum mechanics", J. Mech. Mater. Struct. 4:2 (2009), 281-292.

[dell'Isola et al. 2011] F. dell'Isola, P. Seppecher, and A. Madeo, "Beyond Euler-Cauchy continua: the structure of contact actions in $N$-th gradient generalized continua: a generalization of the Cauchy tetrahedron argument", pp. 17-106 in Variational models and methods in solid and fluid mechanics, edited by F. dell'Isola and S. Gavrilyuk, CISM Courses and Lectures 535, Springer, 2011.

[dell'Isola et al. 2012] F. dell'Isola, P. Seppecher, and A. Madeo, "How contact interactions may depend on the shape of Cauchy cuts in $N$ th gradient continua: approach 'à la D'Alembert"', $Z$. Angew. Math. Phys. 63:6 (2012), 1119-1141.

[dell'Isola et al. 2015a] F. dell'Isola, U. Andreaus, and L. Placidi, "At the origins and in the vanguard of peridynamics, non-local and higher-gradient continuum mechanics: an underestimated and still topical contribution of Gabrio Piola", Math. Mech. Solids 20:8 (2015), 887-928.

[dell'Isola et al. 2015b] F. dell'Isola, P. Seppecher, and A. Della Corte, "The postulations á la D'Alembert and á la Cauchy for higher gradient continuum theories are equivalent: a review of existing results", Proc. A. 471:2183 (2015), 20150415.

[dell' Isola et al. 2017] F. dell'Isola, A. Della Corte, and I. Giorgio, "Higher-gradient continua: the legacy of Piola, Mindlin, Sedov and Toupin and some future research perspectives", Math. Mech. Solids 22:4 (2017), 852-872.

[Dvorkin and Goldschmit 2006] E. N. Dvorkin and M. B. Goldschmit, Nonlinear continua, Springer, 2006.

[Eringen 1980] A. C. Eringen, Mechanics of continua, 2nd ed., Krieger, 1980.

[Eugster 2015a] S. Eugster, Geometric continuum mechanics and induced beam theories, Lecture Notes in Applied and Computational Mechanics 75, Springer, 2015.

[Eugster 2015b] S. R. Eugster, "An intrinsic geometric formulation of the equilibrium equations in continuum mechanics", Proc. Appl. Math. Mech. 15:1 (2015), 289-290.

[Eugster and dell'Isola 2017a] S. R. Eugster and F. dell'Isola, "Exegesis of the introduction and Sect. I from "Fundamentals of the mechanics of continua" by E. Hellinger", Z. Angew. Math. Mech. 97:4 (2017), 477-506.

[Eugster and dell'Isola 2017b] S. R. Eugster and F. dell'Isola, "Exegesis of Sect. II and III.A from "Fundamentals of the mechanics of continua" by E. Hellinger", Z. Angew. Math. Mech. (online publication July 2017).

[Eugster and dell'Isola 2017c] S. R. Eugster and F. dell'Isola, "Exegesis of Sect. III.B from "Fundamentals of the mechanics of continua" by E. Hellinger", Z. Angew. Math. Mech. (online publication September 2017).

[Germain 1972] P. Germain, "Sur l'application de la méthode des puissances virtuelles en mécanique des milieux continus”, C. R. Acad. Sci. Paris 274 (1972), A1051-A1055.

[Germain 1973a] P. Germain, "La méthode des puissances virtuelles en mécanique des milieux continus, I: Théorie du second gradient”, J. Mécanique 12 (1973), 235-274.

[Germain 1973b] P. Germain, "The method of virtual power in continuum mechanics, II: Microstructure”, SIAM J. Appl. Math. 25:3 (1973), 556-575.

[Germain 1986] P. Germain, Mécanique, vol. I, École Polytechnique, 1986. 
[Glocker 2001] C. Glocker, Set-valued force laws: dynamics of non-smooth systems, Lecture Notes in Applied Mechanics 1, Springer, 2001.

[Green and Zerna 1954] A. E. Green and W. Zerna, Theoretical elasticity, Oxford University, 1954.

[Gurtin 1981] M. E. Gurtin, An introduction to continuum mechanics, Mathematics in Science and Engineering 158, Academic, 1981.

[Hamel 1912] G. Hamel, Elemetare Mechanik: ein Lehrbuch, Teubner, 1912.

[Hamel 1927] G. Hamel, "Die Axiome der Mechanik", Kapitel 1, pp. 1-42 in Grundlagen der Mechanik: Mechanik der Punkte und starren Körper, edited by R. Grammel, Handbuch der Physik V, Springer, 1927.

[Hamel 1967] G. Hamel, Theoretische Mechanik: eine einheitliche Einführung in die gesamte Mechanik, Grundlehren der Mathematischen Wissenschaften 57, Springer, 1967.

[Haupt 2002] P. Haupt, Continuum mechanics and theory of materials, 2nd ed., Springer, 2002.

[Hellinger 1914] E. Hellinger, "Die allgemeinen Ansätze der Mechanik der Kontinua”, pp. 602-694 in Enzyklopädie der Mathematischen Wissenschaften mit Einschluss ihrer Anwendungen, vol. IV-4: Mechanik, edited by F. Klein and C. Müller, Teubner, 1914.

[Hilbert 1901] D. Hilbert, "Mathematische Probleme", Arch. Math. Phys. 3:1 (1901), 44-63, 213237.

[Hilbert 1902] D. Hilbert, "Mathematical problems", Bull. Amer. Math. Soc. 8:10 (1902), 437-479.

[Holzapfel 2000] G. A. Holzapfel, Nonlinear solid mechanics: a continuum approach for engineering, Wiley, 2000.

[Kirchhoff 1876] G. Kirchhoff, Vorlesungen über mathematische Physik, Teubner, 1876.

[Kühnel 2013] W. Kühnel, Differentialgeometrie: Kurven - Flächen - Mannigfaltigkeiten, 6th ed., Springer, 2013.

[Lagrange 1788] J.-L. Lagrange, Méchanique analytique, Desaint, 1788.

[Lamé 1852] G. Lamé, Leçons sur la théorie mathématique de l'élasticité des corps solides, École Polytechnique, 1852.

[Lee 2013] J. M. Lee, Introduction to smooth manifolds, 2nd ed., Graduate Texts in Mathematics 218, Springer, 2013.

[Lemaitre and Chaboche 1990] J. Lemaitre and J.-L. Chaboche, Mechanics of solid materials, Cambridge University, 1990.

[Liu 2002] I.-S. Liu, Continuum mechanics, Springer, 2002.

[Macvean 1968] D. B. Macvean, "Die Elementararbeit in einem Kontinuum und die Zuordnung von Spannungs- und Verzerrungstensoren”, Z. Angew. Math. Phys. 19:2 (1968), 157-185.

[Malvern 1965] L. E. Malvern, Introduction to the mechanics of a continuous medium, Prentice-Hall, 1965.

[Mariano and Galano 2015] P. M. Mariano and L. Galano, Fundamentals of the mechanics of solids, Springer, 2015.

[Maugin 1980] G. A. Maugin, "The method of virtual power in continuum mechanics: application to coupled fields", Acta Mech. 35:1-2 (1980), 1-70.

[Müller and Timpe 1914] C. H. Müller and A. Timpe, "Die Grundgleichungen der mathematische Elastizitätstheorie", pp. 1-54 in Enzyklopädie der Mathematischen Wissenschaften mit Einschluss ihrer Anwendungen, vol. IV-4: Mechanik, edited by F. Klein and C. Müller, Teubner, 1914.

[Munkres 2000] J. R. Munkres, Topology, 2nd ed., Prenctice Hall, 2000. 
[Murnaghan 1937] F. D. Murnaghan, "Finite deformations of an elastic solid", Amer. J. Math. 59:2 (1937), 235-260.

[Noll 1959] W. Noll, "The foundations of classical mechanics in the light of recent advances in continuum mechanics", pp. 266-281 in The axiomatic method: with special reference to geometry and physics (Berkeley, CA, 1957-1958), edited by L. Henkin et al., North-Holland, 1959.

[Noll 1974] W. Noll, "La mécanique classique, basée sur un axiome d'objectivité", pp. 135-144 in The foundations of mechanics and thermodynamics, Springer, 1974.

[Ogden 1997] R. W. Ogden, Nonlinear elastic deformations, Dover, 1997.

[Piola 1832] G. Piola, "La meccanica de' corpi naturalmente estesi: trattata col calcolo delle variazioni”, pp. 201-236 in Opuscoli matematici e fisici di diversi autori, vol. I, Giusti, 1832.

[Piola 1848] G. Piola, "Intorno alle equazioni fondamentali del movimento di corpi qualsivogliono, considerati secondo la naturale loro forma e costituzione", pp. 1-186 in Memorie di matematica e fisica della Società italiana delle scienze, vol. XXIV-1, Camera, 1848.

[Piola 2014] G. Piola, The complete works of Gabrio Piola, vol. I, edited by F. dell'Isola et al., Advanced Structured Materials 38, Springer, 2014.

[Poisson 1829] M. Poisson, "Mémoire sur l'équilibre et le mouvement des corps élastique", Mém. Acad. Roy. Sci. 8 (1829), 357-570.

[Salençon 2000] J. Salençon, Mécanique des milieux continus, vol. I: Concepts généraux, École Polytechnique, 2000.

[Salençon 2001] J. Salençon, Handbook of continuum mechanics: general concepts, thermoelasticity, Springer, 2001.

[Schwartz 1951] L. Schwartz, Théorie des distributions, vol. II, Publications de l'Institut de Mathématique de l’Université de Strasbourg 10, Hermann, 1951.

[Sedov 1972] L. I. Sedov, A Course in Continuum Mechanics, vol. 4: Basic equations and analytical techniques, Wolters-Noordhoff, 1972.

[Segev 1986] R. Segev, "Forces and the existence of stresses in invariant continuum mechanics", $J$. Math. Phys. 27:1 (1986), 163-170.

[Spencer 2004] A. J. M. Spencer, Continuum mechanics, Dover, 2004.

[Strichartz 1994] R. S. Strichartz, A guide to distribution theory and Fourier transforms, CRC, 1994.

[Thomson and Tait 1867] W. Thomson and P. G. Tait, "Abstract dynamics", division II, pp. 438-849 in Treatise on natural philosophy, vol. 1, Oxford University, 1867.

[Timoshenko and Goodier 1951] S. Timoshenko and J. N. Goodier, Theory of Elasticity, 2nd ed., McGraw-Hill, 1951.

[Toupin 1964] R. A. Toupin, “Theories of elasticity with couple-stress", Arch. Rational Mech. Anal. 17 (1964), 85-112.

[Truesdell 1991] C. A. Truesdell, A first course in rational continuum mechanics, vol. 1: General concepts, 2nd ed., Pure and Applied Mathematics 71, Academic, 1991.

[Truesdell and Noll 1965] C. Truesdell and W. Noll, The non-linear field theories of mechanics, Handbuch der Physik III-3, Springer, 1965.

[Truesdell and Toupin 1960] C. Truesdell and R. Toupin, The classical field theories, Handbuch der Physik III-1, Springer, 1960.

[Volokh 2006] K. Y. Volokh, "Lagrangian equilibrium equations in cylindrical and spherical coordinates", Comput. Mater. Con. 3:1 (2006), 37-42. 
Received 18 Nov 2016. Revised 22 May 2017. Accepted 19 Aug 2017.

SIMON R. EUGSTER: eugster@inm.uni-stuttgart.de Institute for Nonlinear Mechanics, University of Stuttgart, Stuttgart, Germany CHRISTOPH GLOCKER: glocker@imes.mavt.ethz.ch ETH Zürich, Zurich, Switzerland 


\section{Guidelines for Authors}

Authors may submit manuscripts in PDF format on-line at the submission page.

Originality. Submission of a manuscript acknowledges that the manuscript is original and and is not, in whole or in part, published or under consideration for publication elsewhere. It is understood also that the manuscript will not be submitted elsewhere while under consideration for publication in this journal.

Language. Articles in MEMOCS are usually in English, but articles written in other languages are welcome.

Required items. A brief abstract of about 150 words or less must be included. It should be selfcontained and not make any reference to the bibliography. If the article is not in English, two versions of the abstract must be included, one in the language of the article and one in English. Also required are keywords and a Mathematics Subject Classification or a Physics and Astronomy Classification Scheme code for the article, and, for each author, postal address, affiliation (if appropriate), and email address if available. A home-page URL is optional.

Format. Authors are encouraged to use $\mathrm{IAT}_{\mathrm{E} X}$ and the standard amsart class, but submissions in other varieties of $\mathrm{T}_{\mathrm{E}} \mathrm{X}$, and exceptionally in other formats, are acceptable. Initial uploads should normally be in PDF format; after the refereeing process we will ask you to submit all source material.

References. Bibliographical references should be complete, including article titles and page ranges. All references in the bibliography should be cited in the text. The use of $\mathrm{BIBT}_{\mathrm{E}} \mathrm{X}$ is preferred but not required. Tags will be converted to the house format, however, for submission you may use the format of your choice. Links will be provided to all literature with known web locations and authors are encouraged to provide their own links in addition to those supplied in the editorial process.

Figures. Figures must be of publication quality. After acceptance, you will need to submit the original source files in vector graphics format for all diagrams in your manuscript: vector EPS or vector PDF files are the most useful.

Most drawing and graphing packages - Mathematica, Adobe Illustrator, Corel Draw, MATLAB, etc. - allow the user to save files in one of these formats. Make sure that what you are saving is vector graphics and not a bitmap. If you need help, please write to graphics@msp.org with as many details as you can about how your graphics were generated.

Bundle your figure files into a single archive (using zip, tar, rar or other format of your choice) and upload on the link you been provided at acceptance time. Each figure should be captioned and numbered so that it can float. Small figures occupying no more than three lines of vertical space can be kept in the text ("the curve looks like this:"). It is acceptable to submit a manuscript with all figures at the end, if their placement is specified in the text by means of comments such as "Place Figure 1 here". The same considerations apply to tables.

White Space. Forced line breaks or page breaks should not be inserted in the document. There is no point in your trying to optimize line and page breaks in the original manuscript. The manuscript will be reformatted to use the journal's preferred fonts and layout.

Proofs. Page proofs will be made available to authors (or to the designated corresponding author) at a Web site in PDF format. Failure to acknowledge the receipt of proofs or to return corrections within the requested deadline may cause publication to be postponed. 
Mathematics and Mechanics of Complex Systems vol. 5 no. 3-4

Forms of the dissipation function for a class of viscoplastic 217 models

Massimo Cuomo

Tumor growth modelling by cellular automata

Ruben Interian, Reinaldo Rodríguez-Ramos, Fernando

Valdés-Ravelo, Ariel Ramírez-Torres, Celso C. Ribeiro and Aura Conci

A variational formulation for fuzzy analysis in continuum 261 mechanics

Rolf Mahnken

On the notion of stress in classical continuum mechanics

Simon R. Eugster and Christoph Glocker

MEMOCS is a journal of the International Research Center for the Mathematics and Mechanics of Complex Systems at the Università dell' Aquila, Italy.

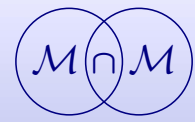

